\title{
TWISTED MAGNETIZATION PHASES IN ORBITAL-DOMINANT RARE-EARTH NITRIDES
}

BY

\section{JAMES FRANCIS MCNULTY}

\author{
A thesis \\ submitted to the Victoria University of Wellington \\ in fulfilment of the requirements for the degree of \\ Doctor of Philosophy \\ in Physics
}

Victoria University of Wellington 2015 



\section{Abstract}

In this thesis we investigate the magnetic properties of $\mathrm{NdN}$ and $\mathrm{SmN}$, members of the rare-earth nitrides, a series of intrinsic ferromagnetic semiconductors. In rare-earth systems, the strong spin-orbit coupling of the partially filled $4 f$ shell ensures that there is a substantial orbital contribution to the ferromagnetic moment, in contrast to many transition metal systems where the orbital moment is usually quenched. In $\mathrm{SmN}$ and $\mathrm{NdN}$ the orbital moment actually exceeds the spin moment, and the resulting orbital dominant magnetization allows for the fabrication of a magnetic heterostructures showing novel behavior.

We report a new theoretical study of the magnetic properties on both $\mathrm{SmN}$ and $\mathrm{NdN}$ by considering the atomic-like $4 f$ electrons. These calculations incorporate spin-orbit coupling, the exchange interaction in a self-consistent mean-field approach, and crystal field interactions in an arbitrary-multiplet point-charge model. Our findings show excellent agreement with the experimentally measured ferromagnetic moments of $\mathrm{SmN}$ and $\mathrm{NdN}$, representing an advance from previous theoretical studies.

We also report an experimental study on $\mathrm{SmN} / \mathrm{GdN}$ heterostructures using the element-resolved method of x-ray magnetic circular dichroism (XMCD) to

probe the magnetism. The competition between the orbital-dominant Zeeman coupling in $\mathrm{SmN}$ and the ferromagnetic spin-based interface exchange with GdN, which has purely a spin moment, results in a twisted magnetization profile. The depth profile of the magnetization derived from XMCD measurements showed good agreement with an analytical model developed to describe the competing interactions.

In a second study, a superlattice of $\mathrm{NdN} / \mathrm{GdN}$ was investigated via XMCD 
and standard magnetometry techniques. A twisted magnetization was shown to be present due to the same mechanism as in the $\mathrm{SmN} / \mathrm{GdN}$ system. By varying the maximum applied field and temperature, twisted phases were shown to develop in both GdN and NdN layers. These twisted phases in orbitaldominant ferromagnetic semiconductors represent a departure from previously explored spin-dominant metallic systems displaying similar twisted phases. 


\section{Acknowledgements}

This thesis represents more than just an academic work. It also serves as a souvenir of my time in New Zealand, which has been truly remarkable. Many people have supported me in ways large and small over the years, scientifically and personally, and here I would like to thank them.

First of all, I would like to thank my supervisors Ben Ruck and Joe Trodahl for their support, encouragement, and good humor. I have learned a great deal from the illuminating scientific discussions with Ben and Joe over the years, and feel lucky to have had such excellent supervisors.

I have benefited greatly from working with my colleagues in the group, including Harry Warring, Tanmay Maity, Luis Figueras, Franck Natali, and Eva Anton. Simon Granville and Shen Chong were of invaluable help with measurements at the Robinson Research institute. The expert assistance from the beamline scientists was also invaluable: Marcio Medeiros-Soares and Nick Brookes at the ESRF, Motohiro Suzuki and Masaichiro Mizumaki at SPring-8.

My officemates Brendan Darby, Peter Hauer, Walter Somerville, Stefan Meyer, Camille Artur, and Chris Galloway made the office an enjoyable place to work. I acknowledge other friends and colleagues who made my time here so enjoyable: Stephanie Droste, Natalie Plank, Leah Graham, Thom Minnee, Stefan Kuczera, Hani Hatami, Conor Burke, Pablo Hernandez, Baptiste Auguié, and many others. A special mention must go to Andrew Preston and Shaun Preston, great friends and flatmates who introduced me to the Kiwi way of life.

I would like to thank Eva Anton especially for her efforts in proofreading, and being so supportive through the writing process.

Finally, I recognize my parents and family, who provided so much support 
on the journey leading to this point. To my father, who got me interested in science in the first place, and my mother, who has been a steady source of encouragement over the years, thank you. 
"The time has come," the Walrus said,

"To talk of many things:

Of shoes-and ships-and sealing-wax-

Of cabbages-and kings-

And why the sea is boiling hot-

And whether pigs have wings."

- Lewis Carroll, from "The Walrus and the Carpenter" 


\section{Contents}

1 Introduction 1

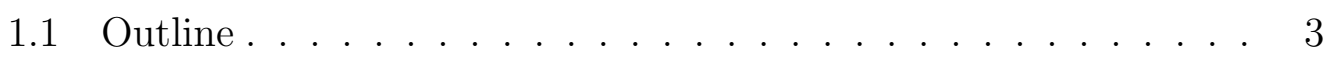

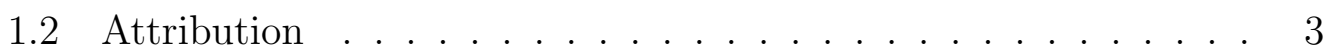

1.3 Publications . . . . . . . . . . . . . . . . . 4

2 Theoretical Background $\quad 7$

2.1 Electronic Structure . . . . . . . . . . . . . . . . . 7

2.2 Hund's Rules . . . . . . . . . . . . . . . . . . . . 11

2.3 Rare-earth Magnetism . . . . . . . . . . . . . . . . . 13

2.3.1 Atomic magnetism . . . . . . . . . . . . 13

2.3.2 Paramagnetism . . . . . . . . . . . . . 15

2.3.3 Van Vleck paramagnetism . . . . . . . . . . . . . 20

2.3.4 Ferromagnetism . . . . . . . . . . . . . . . . . 21

2.3.5 The crystal field . . . . . . . . . . . . . . . 26

2.4 Exchange Springs . . . . . . . . . . . . . . . . . . . 31

3 Experimental Techniques $\quad 35$

3.1 Synchrotron Techniques . . . . . . . . . . . . . 35

3.1.1 M-edge absorption . . . . . . . . . . . . . . 39

3.1.2 L-edge absorption . . . . . . . . . . . . . . . . . . . . 41

3.1.3 Detection methods . . . . . . . . . . . . . . . . 41

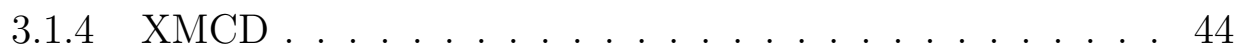

3.1.5 The two-step XMCD model . . . . . . . . . . . . . . 44

3.1.6 Rare-earth L-edge XMCD . . . . . . . . . . . . . 46

3.1.7 Rare-earth M-edge XMCD . . . . . . . . . . . . . . 47 
3.1 .8 XMCD sum rules . . . . . . . . . . . . . . 50

3.2 Thin Film Growth . . . . . . . . . . . . . . . . 51

$4 \quad \mathrm{SmN}$ and NdN Magnetism 53

4.1 Introduction . . . . . . . . . . . . . . . . . . . . 53

$4.2 \mathrm{SmN}$ Magnetism . . . . . . . . . . . . . . . . . 54

4.2.1 Experimental background . . . . . . . . . . . . 54

4.2.2 $\mathrm{SmN}$ calculations . . . . . . . . . . . . . 55

4.2.3 Calculation procedure . . . . . . . . . . . . . 58

4.2.4 Excited states . . . . . . . . . . . . . . . . . . 61

4.2.5 SmN crystal field . . . . . . . . . . . . . . . . . . . . . . . . . 71

4.3 NdN Magnetism . . . . . . . . . . . . . . . . . 71

4.3.1 Magnetic moment calculation . . . . . . . . . . 75

$4.3 .2 \mathrm{NdN}$ crystal field . . . . . . . . . . . . . . 77

4.3.3 Effects of the crystal field on $T_{C} \ldots \ldots . . . . . .884$

4.4 Conclusion . . . . . . . . . . . . . . . . . . 88

5 Twisted Magnetization in $\mathrm{SmN} / \mathrm{GdN} \quad 91$

5.1 Introduction . . . . . . . . . . . . . . . . . 91

5.2 Experimental Details . . . . . . . . . . . . . . . . 94

5.3 L-edge XMCD Results . . . . . . . . . . . . . . . . . . . . 97

5.4 M-edge XMCD Results . . . . . . . . . . . . . . . . . 102

5.5 Analysis and Discussion . . . . . . . . . . . . . 107

5.6 Conclusion and Outlook . . . . . . . . . . . . . . 112

6 Twisted Magnetization in a NdN/GdN Superlattice 115

6.1 Introduction . . . . . . . . . . . . . . . . . 115

6.2 Experimental Details . . . . . . . . . . . . . . 116

6.3 Magnetization Results . . . . . . . . . . . . . . . 117

6.4 XMCD Investigation . . . . . . . . . . . . . . . 126

6.4.1 XMCD experimental details . . . . . . . . . . 126

6.4.2 $\mathrm{Gd} \mathrm{L}_{2,3}$ and $\mathrm{Nd} \mathrm{L}_{2}$ edges . . . . . . . . . . . . 127

6.4 .3 XMCD hysteresis . . . . . . . . . . . . 130

6.5 Discussion . . . . . . . . . . . . . . . . . 133 
6.6 Conclusion and Outlook . . . . . . . . . . . . . 136

$\begin{array}{llr}7 & \text { Conclusions and Outlook } & 139\end{array}$

7.1 Results . . . . . . . . . . . . . . . . . . . . . . . 139

7.2 Future Outlook . . . . . . . . . . . . . . . 140

$\begin{array}{lr}\text { Appendices } & 145\end{array}$

A Matrix Elements of Irreducible Tensor Operators $\quad 147$

A.1 Irreducible Tensor Operators . . . . . . . . . . . . . . . . . . . 147

A.2 The Wigner-Eckart Theorem . . . . . . . . . . . . . . . . . 148

A.3 Matrix Elements Between Arbitrary Multiplets . . . . . . . . . . 151

$\begin{array}{ll}\text { B Free Energy Minimization } & 157\end{array}$

B.1 Extremizing a Functional . . . . . . . . . . . . . . 157

B.2 Explicit Solution for the Twisted Phase . . . . . . . . . . 159

$\begin{array}{lr}\text { References } & 162\end{array}$ 


\section{CONTENTS}




\section{Chapter 1}

\section{Introduction}

Research into rare-earth nitrides (RENs) has experienced a surge of interest over the past ten years, spurred by the theoretical prediction [1] that some of the RENs are half-metallic ferromagnets, which suggested the application of RENs in spin-filters. Subsequent experimental investigations have found that a majority of the RENs are in fact intrinsic ferromagnetic semiconductors [2-8]. This makes the RENs quite distinct from the more well known dilute magnetic semiconductors (DMS) such as (Ga,Mn)As [9]. In DMS systems, the host semiconductor is doped with magnetic impurity ions to achieve both ferromagnetism and semiconducting behavior. The Curie temperature and conductivity are thus strongly dependent on the spatial distribution of these impurity ions, which is difficult to control. The difficulty in independently manipulating the magnetic and electronic degrees of freedom is thus a large drawback in DMS systems.

The RENs derive their magnetic properties from the partially filled $4 f$ shell of the rare-earth elements, which consists of 14 distinct single-electron states, resulting in widely varying magnetic properties as the occupation increases across the series. The magnetic order is largely insensitive to the number of free carriers arising from nitrogen vacancy defects for a wide range of concentrations [10]. This is due to the localized exchange mechanism responsible for ferromagnetic ordering, in contrast to longer range carrier-mediated exchange commonly found in metallic rare-earth systems [11-13]. This localized exchange in the RENs also leads to rather low ordering temperatures compared 


\section{Chapter 1 Introduction}

to metallic rare-earth systems, requiring liquid He temperatures. But most importantly, semiconducting and ferromagnetic behavior coincide, and electronic structure calculations show spin-polarized bands with conduction and valence band edges of the majority spin [14].

These properties have led to the integration of the rare-earth nitrides in spintronic devices, with GdN incorporated as a barrier layer in a spin-filter Josephson junction [15], and $\mathrm{DyN}$ as a barrier layer in a magnetic tunnel junction [16]. Incorporating various RENs into semiconducting magnetic tunnel junctions for use in magnetoresistive random access memory elements (MRAM) [17] is also an intriguing possibility due to the large spin polarizations and semiconducting nature of the RENs.

While the device implications of the rare-earth nitrides are one important motivation for studying them, fundamental physical questions about the RENs are not yet resolved. GdN is the most thoroughly investigated of the series, with numerous theoretical and experimental studies into the electronic and magnetic structure, however for the other RENs there are far fewer reports. Indeed, for the majority of the RENs there is only one recent band structure calculation which also provides a calculation of magnetic moments [14].

Understanding the magnetic moment is non-trivial, as an unquenched orbital contribution to the magnetization may result in a net magnetization parallel to the orbital moment and antiparallel to the spin moment in the first half of the rare-earth series. The presence of unquenched, orbital-dominant moments then complicates the study of magnetic heterostructures composed of RENs. In this thesis we calculate the magnetic moments of SmN and $\mathrm{NdN}$ within crystal-field theory in order to better understand the interplay between orbital and spin contributions to the magnetization. The crystal field calculations represent an alternative approach to the existing density functional theory calculations carried out on $\mathrm{SmN}$ and $\mathrm{NdN}[14,18]$. We find good agreement with experimental data within this crystal field approach.

The primary experimental work on RENs so far has been on homogeneous RENs, with only two publications on REN heterostructures, by Anton et al. [19] and McNulty et al. [20], the latter of which is part of this thesis (Chapter $5)$. The study of these heterostructures is a necessary step for exploring poten- 
tial device applications, but more fundamentally allows us to probe unexplored interface effects in REN-based heterostructures. In this thesis we carried out magnetic studies using element specific x-ray techniques as well as more common magnetization measurements in heterostructures composed of SmN/GdN and $\mathrm{NdN} / \mathrm{GdN}$ layers. In these heterostructures we demonstrate the existence of a "twisted" or inhomogeneous magnetic phase occurring at interfaces. These twisted phases are shown to be a consequence of the unique rare-earth magnetism, specifically the orbital-dominant magnetism of $\mathrm{NdN}$ and $\mathrm{SmN}$ in combination with the spin-only magnetic moment of GdN. These experimental studies, together with the new calculations of the $\mathrm{SmN}$ and NdN magnetic moments provide the first detailed description of REN-based heterostructures.

\subsection{Outline}

This thesis is organized as follows. Chapter 2 contains the theoretical background reviewing the relevant electronic structure of rare-earth ions, and introduces magnetism in the RENs, including an overview of the crystal field calculations. Chapter 3 introduces the primary experimental technique used in this thesis, x-ray magnetic circular dichroism (XMCD), and the sample preparation techniques used. In Chapter 4, the calculation of the magnetic moments of $\mathrm{SmN}$ and $\mathrm{NdN}$ is presented with comparisons to recent and published experimental data. Chapter 5 reports on x-ray magnetic circular dichroism experiments on a $\mathrm{SmN} / \mathrm{GdN}$ superlattice as well as bilayers of $\mathrm{SmN} / \mathrm{GdN}$ and $\mathrm{SmN} / \mathrm{LaN} / \mathrm{GdN}$. Chapter 6 is a study of a NdN/GdN superlattice using x-ray magnetic circular dichroism and SQUID based magnetometry. And finally, Chapter 7 summarizes the conclusions from this thesis and provides an outlook for future research.

\subsection{Attribution}

Much of the experimental work was done in collaboration with others:

- $S m N / G d N L$-edge XMCD: This data is presented in 
Chapter 5. Measurements were carried out by E.-M. Anton, B. J. Ruck, C. Meyer, H. J. Trodahl, F. Wilhelm, A. Rogalev at the European synchrotron radiation facility (ESRF) in Grenoble, France. Samples were grown by H. Warring and F. Natali.

- M-edge XMCD: Presented in Chapter 5. These measurements were carried out by myself and E. -M. Anton, B. J. Ruck, H. J. Trodahl, M. Medeiros-Soares, N. B. Brookes at the ESRF. I prepared all of the samples for these experiments.

- $N d / G d$ L-edge XMCD: Presented in Chapter 6. The L-edge measurements were performed by myself and E.-M. Anton, H. J. Trodahl, T. Yamada, M. Suzuki, and M. Mizumaki at the SPring 8 Synchrotron facility in Japan. The NdN/GdN superlattice was grown by E.-M. Anton and myself, while the NdN sample was prepared by E.-M. Anton.

- SQUID Measurements: NdN magnetization measurements were carried out by E.-M Anton. Magnetization measurements on the NdN/GdN superlattice, SmN/GdN, and SmN/LaN/GdN samples were carried out by myself. Many of these measurements benefited from the assistance of Simon Granville and Shen Chong.

- Calculations: The calculations in Chapter 4 were programmed in Python by myself, as were the calculations in Chapter 5 .

\subsection{Publications}

1. J. F. McNulty, E. -M. Anton, B. J. Ruck, F. Natali, H. Warring, F. Wilhem, A. Rogalev, M. Madeiros-Soares, N. Brookes, and H. J. Trodahl. Twisted magnetization in the orbital-dominant ferromagnet $S m N$ in GdN/SmN heterostructures. Phys. Rev. B 91, 174426 (2015).

2. B. Chen, J. Laverock, D. Newby Jr, J. F. McNulty, K. E. Smith, PA. Glans, J-H. Guo, R-M. Qiao, W.-L. Yang, M.R. Lees, L. D. Tung, 
R. P. Singh, and G. Balakrishnan. Effects of rare-earth size on the electronic structure of $L a_{1-x} L u_{x} V O_{3}$. J. Phys.: Condens. Matter 27105503 (2015).

3. H. Warring, B. J. Ruck, J. F. McNulty, E. -M. Anton, S. Granville, A. Koo, B. Cowie, and H. J. Trodahl. YbN: An intrinsic semiconductor with antiferromagnetic exchange. Phys. Rev. B. 245206 (2014).

4. J. Laverock, L. F. J. Piper, A. R. H. Preston, B. Chen, J. F. McNulty, K. E. Smith, S. Kittiwatanakul, J. W. Lu, S. A. Wolf, P. -A. Glans, J. -H Guo. Strain dependence of bonding and hybridization across the metal-insulator transition of $V_{2}$. Phys. Rev. B 85 081104(R) (2012).

5. D. J. Payne, M. D. M. Robinson, R. G. Egdell, A. Walsh, J. F. McNulty, K. E. Smith, and L. F. J. Piper. The nature of electron lone pairs in $\mathrm{BiVO}_{4}$. Appl. Phys. Lett. 98, 212110 (2011)

6. J. Laverock, A. R. H. Preston, B. Chen, J. F. McNulty, K. E. Smith, L. F. J. Piper, P. -A. Glans, and J. -H Guo. Orbital anisotropy and lowenergy excitations of the quasi one-dimensional conductor $\beta-\mathrm{Sr}_{0.17} \mathrm{~V}_{2} \mathrm{O}_{5}$.

Phys. Rev. B. 84, 155103 (2011)

7. J. H. Richter, B. J. Ruck, M. Simpson, F. Natali, N. O. V. Plank, M. Azeem, H. J. Trodahl, A. R. H. Preston, B. Chen, J. F. McNulty, K. E. Smith, A. Tadich, B. Cowie, A. Svane, M. van Schilfgaarde, W. R. L. Lambrecht. Electronic structure of EuN: Growth, spectroscopy, and theory. Phys. Rev. B. 84, 235120 (2011)

\section{Patents}

1. B. J. Ruck, H. J. Trodahl, E. -M. Anton, F. Natali, J. F. McNulty, S. Granville. Magnetic materials and devices comprising rare-earth nitrides. N.Z. Patent 623343, 2014. 
Chapter 1 Introduction 


\section{Chapter 2}

\section{Theoretical Background}

\subsection{Electronic Structure}

In order to understand the magnetic properties of the rare-earth nitrides, we must first understand the electronic configuration of the partially filled $4 f$ shell, which is the origin of the magnetism in rare-earth systems. To do this we proceed by examining the Hamiltonian of the $4 f$ electrons. Later we will see that the magnetic properties of the RENs can to a large degree be understood by an atomic picture of the $4 f$ shell.

We begin by considering a single $4 f$ electron in a rare-earth ion with wave function $\psi_{i}$. In the potential field of the ion, the electron can described by the time-independent Schrödinger equation, $\mathscr{H}_{i} \psi_{i}=E_{i} \psi_{i}$, where

$$
\mathscr{H}_{i}=-\frac{\hbar^{2}}{2 m_{e}} \nabla_{i}^{2}+V\left(r_{i}\right),
$$

with $r_{i}=\left|\mathbf{r}_{i}\right|$ is the position vector of the electron and $m_{e}$ is the electron mass. $V\left(r_{i}\right)=-Z^{*} e / r_{i}$ represents the Coulomb potential energy between the $i$-th $4 f$ electron in the ion, and $Z^{*}$ represents the effective charge of the ion, including the filled electronic shells. The rare-earth elements are most commonly found in the trivalent $(3+)$ state, and this is found to be the case in most of the RENs. The Schrödinger equation in this form does not adequately describe the $4 f$ electrons, however, as relativistic effects must be included. Beginning with atoms having atomic number $Z=10$, binding energies require relativistic 


\section{Chapter 2 Theoretical Background}

corrections, and for wave functions relativistic corrections must be considered for atoms heavier than $Z=30$ [21]. Therefore, from the outset we must consider relativistic effects, as the rare-earth elements start with Lanthanum, with $Z=57$.

We can obtain the correct description by considering the relativistic Dirac equation, but for our purposes it is much easier to use only the lowest order terms from the expansion of the Dirac equation in powers of $v / c$. The Hamiltonian obtained from this expansion is given by [21]:

$$
\begin{aligned}
\mathscr{H}_{i}= & -\frac{\hbar^{2}}{2 m_{e}} \nabla_{i}^{2}+V\left(r_{i}\right)-\frac{\alpha^{2}}{4}\left(\frac{\hbar}{2 m_{e}} \nabla_{i}^{2}\right)^{2}-\frac{\alpha^{2}}{4}\left(\frac{\mathrm{d} V\left(r_{i}\right)}{\mathrm{d} r}\right) \frac{\partial}{\partial r} \\
& +\frac{\alpha^{2}}{2} \frac{1}{r_{i}}\left(\frac{\mathrm{d} V\left(r_{i}\right)}{\mathrm{d} r}\right) \mathbf{l}_{i} \cdot \mathbf{s}_{i},
\end{aligned}
$$

where $\alpha=e^{2} / \hbar c \approx 1 / 137$ is the fine structure constant. This equation is the same as the Schrödinger equation 2.1 but with three additional terms. The third and fourth terms only give a rigid shift to the absolute energy levels, which is not important for our purposes. The last term contains $\mathbf{l}_{i} \cdot \mathbf{s}_{i}$, which represent the orbital and spin angular momentum operators of the $i$ th $4 f$ electron. This term is known as the spin-orbit coupling term and plays a major role in the electronic configuration in the rare-earths. The coefficient

$$
\lambda\left(\mathbf{r}_{i}\right)=\frac{\alpha^{2}}{2} \frac{1}{r_{i}}\left(\frac{\mathrm{d} V\left(r_{i}\right)}{\mathrm{d} r}\right)
$$

is the spin-orbit coupling parameter. This parameter is large for tightly bound orbitals with small radii, such as the $4 f$ electrons, due to the dependence of $V(r)$ on $r^{-1}$. Figure 2.1 shows the expectation value $\langle r\rangle$ for various orbitals as a function of atomic number $Z$. The $4 f$ radii can be seen to be smaller than all orbitals with principal quantum number $n \geqslant 5$, which includes the completely filled $5 s$ and $5 p$ shells, as well as valence $6 s$ and $5 d$ states in the rare-earths. These larger orbitals keep the $4 f$ orbitals shielded from surrounding ions, so that they remain atomic-like even in a solid [2].

To consider all the electrons in the $4 f$ shell, we must add together each single electron Hamiltonian, and in addition we must also include the Coulomb 


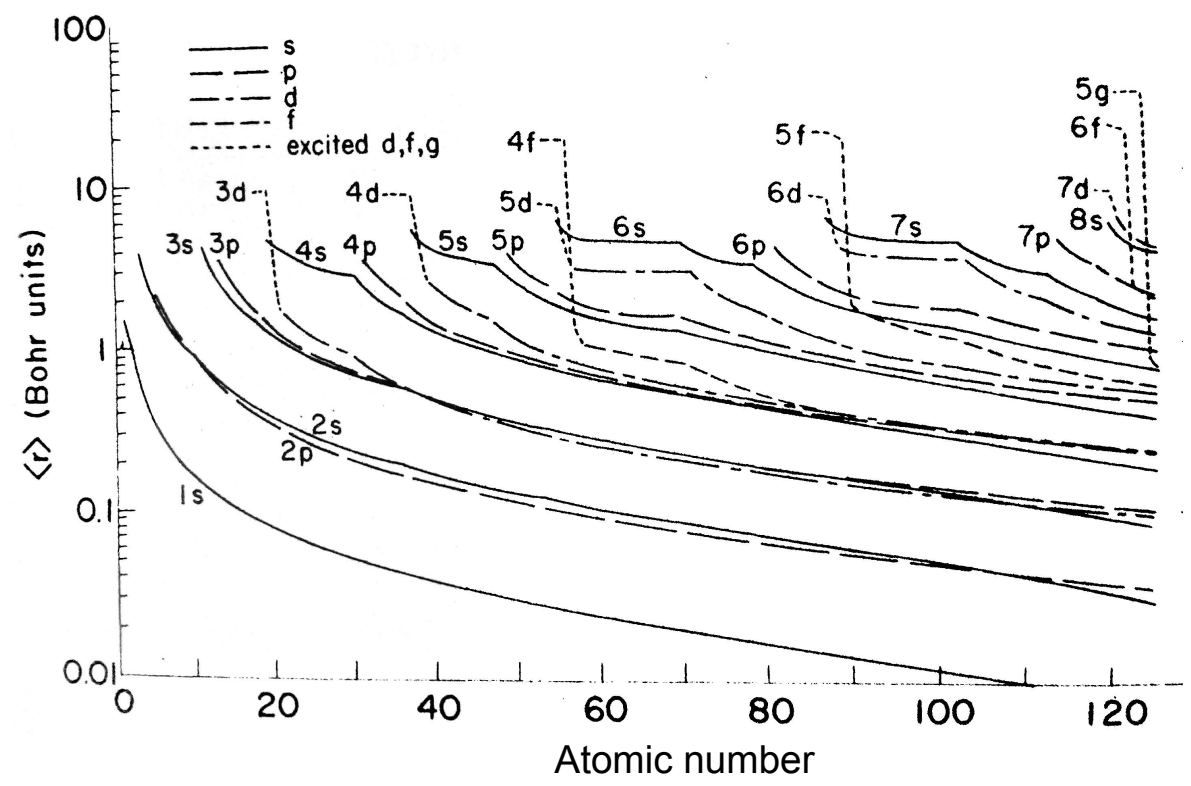

Figure 2.1: Expectation values of $r$ for various atomic orbitals as a function of atomic number $Z$, calculated within a Hartree-Fock scheme with relativistic corrections, taken from Cowan [21].

interaction between pairs of electrons. ${ }^{1}$ The total Hamiltonian for the partially filled $4 f$ shell is then

$$
\mathscr{H}_{4 f}=-\frac{\hbar^{2}}{2 m_{e}} \sum_{i} \nabla_{i}^{2}-\sum_{i} V\left(r_{i}\right)+\frac{1}{2} \sum_{i \neq j} \frac{e^{2}}{\left|\mathbf{r}_{j}-\mathbf{r}_{i}\right|}+\sum_{i} \lambda\left(\mathbf{r}_{i}\right) \mathbf{l}_{i} \cdot \mathbf{s}_{i} .
$$

The first two terms are the same for all the electrons in the same shell, and just provide an overall rigid shift to the energy levels. The effective Hamiltonian for the $4 f$ shell can finally be reduced to

$$
\mathscr{H}_{4 f}=\frac{1}{2} \sum_{i \neq j} \frac{e^{2}}{\left|\mathbf{r}_{j}-\mathbf{r}_{i}\right|}+\sum_{i} \lambda\left(\mathbf{r}_{i}\right) \mathbf{l}_{i} \cdot \mathbf{s}_{i}
$$

where the first term is the electron-electron interaction $\left(\mathscr{H}_{e-e}\right)$, and the last term is the total spin-orbit interaction $\left(\mathscr{H}_{S O}\right)$. These two terms determine the relative energies of different electron configurations of the partially filled

\footnotetext{
${ }^{1}$ Here we neglect terms $\mathbf{s}_{\mathbf{i}} \cdot \mathbf{s}_{\mathbf{j}}$, and $\mathbf{l}_{i} \cdot \mathbf{l}_{j}$, which are much smaller than the spin-orbit interaction term $[21,22]$
} 


\section{Chapter 2 Theoretical Background}

If shell. The electron-electron interaction term $\mathscr{H}_{e-e}$ makes the Hamiltonian impossible to solve in general, thus various approximation schemes must be used, such as Hartree-Fock [23]. Depending on which term is larger, two coupling schemes for the angular momenta are used to describe the Hamiltonian. We note that $\mathscr{H}_{e-e}$ commutes with the operators $\mathbf{L}=\sum_{i} \mathbf{l}_{i}, \mathbf{S}=\sum_{i} \mathbf{s}_{i}$, $\mathbf{J}=\mathbf{L}+\mathbf{S}$ and their $z$ components $L_{z}, S_{z}$ and $J_{z}$, while $\mathscr{H}_{S O}$ commutes with $\mathbf{J}$ and $\mathbf{j}_{\mathbf{i}}=\mathbf{l}_{i}+\mathbf{s}_{i}$, but not $\mathbf{L}$ or $\mathbf{S}$.

When $\mathscr{H}_{e-e}$ is larger than the spin-orbit term, then to a good approximation the stationary states of the full Hamiltonian are the eigenstates of $\mathbf{L}^{2}, \mathbf{S}^{2}, \mathbf{J}^{2}$ and $J_{z}$, with eigenvalues $L(L+1), S(S+1), J(J+1)$ and $M_{J}$, respectively. This is known as the $L S$-coupling or Russell-Saunders coupling. The quantum numbers describing the states are then $L, S, J, M_{J}$.

The second coupling scheme is known as $j j$-coupling, used when $\mathscr{H}_{S O} \gg$ $\mathscr{H}_{e-e}$, so that the full Hamiltonian is close to diagonal when written in terms of $\mathbf{j}_{i}$ and $j_{i}^{z}$. For atoms lighter than $\mathrm{Pb}(Z=82), \mathscr{H}_{e-e}$ dominates, and so the rare-earth $4 f$ shell then falls into the $L S$ coupling regime.

In $L S$ coupling, $\mathscr{H}_{S O}$ can be rewritten in terms of $\mathbf{L}$ and $\mathbf{S}$ as

$$
\mathscr{H}_{S O}=\Lambda \mathbf{L} \cdot \mathbf{S}, \quad(L S \text {-coupling })
$$

where $\Lambda$ is the total spin-orbit coupling parameter for the $4 f$ shell. $^{2}$ In the $L S$ coupling regime, the relative energy of an electron configuration depends only on the quantum numbers $J, L$, and $S$, often represented by a term symbol ${ }^{2 S+1} L_{J}$. The energy of a configuration with a term symbol ${ }^{2 S+1} L_{J}$ is

$$
E_{L S J}=\left\langle{ }^{2 S+1} L_{J}\left|\mathscr{H}_{e-e}+\mathscr{H}_{S O}\right|^{2 S+1} L_{J}\right\rangle
$$

In the absence of spin-orbit coupling, all configurations would have the same energy, with $(2 L+1)(2 S+1)$-fold degeneracy. The spin-orbit coupling partially reduces this degeneracy. The energy levels are split based on the $J$ value, each with degeneracy $2 J+1$.

Fortunately, for $L S$ coupling there exist empirical rules which specify the

\footnotetext{
${ }^{2}$ This assumes that the quantum numbers $L$ and $S$ are fixed, and higher values of $L$ or $S$ are far off in energy.
} 
ground state configuration, known as Hund's rules. These rules accurately give the ground state configuration $(L, S, J)$ of all the rare-earth ions [2]. The ground state is of most interest for us for the magnetic and spectroscopy applications we will use later on.

\subsection{Hund's Rules}

Hund's rules can be heuristically argued by trying to minimize the energy of the atomic Hamiltonian $\mathscr{H}=\mathscr{H}_{e-e}+\mathscr{H}_{S O}$. The rules should be applied in the following order:

1. Maximize $S$

2. Given the maximum $S$, maximize $L$,

3. $J=|L-S|$ for a less than half-filled shell, $J=|L+S|$ for a more than half-filled shell.

The first rule arises from the Pauli exclusion principle, where parallel spins are farther apart, reducing the Coulomb repulsion. Maximizing $L$ follows the same reasoning; for large $L$ the spatial wave function has more nodes and the probability of finding electrons close together is reduced. The third rule comes from considering the spin-orbit coupling. For less than half-filled shells, $\Lambda>0$, so minimizing the magnitude of $J$ will reduce $\mathscr{H}_{S O}$. For a more than half-filled shell, $\Lambda<0$, and thus a maximum value of $J$ reduces $\mathscr{H}_{S O}$.

For example, $\mathrm{Sm}^{3+}$ has five $4 f$ electrons out of the $2(2 l+1)=14$ possible states in the $4 f$ shell. The largest value $S$ can have is given by the maximum value of the $z$ component, $M_{S}$. Since there are only five electrons, they each have the same $z$ component of the spin $m_{s}$, and $M_{S}=\sum m_{s}=5 / 2$, and so $S=5 / 2$. The maximum orbital moment is then found by $M_{L}=\sum m_{l}=$ $3+2+1+0-1=5$, thus $L=5$. Because the shell is less than half filled, $J=|L-S|=5 / 2$.

The term symbol ${ }^{2 S+1} L_{J}$ is unfortunately written using spectroscopic notation for $L$, with $0,1,2,3, \ldots=S, P, D, F \ldots$, thus $\mathrm{Sm}^{3+}$ has the term symbol 


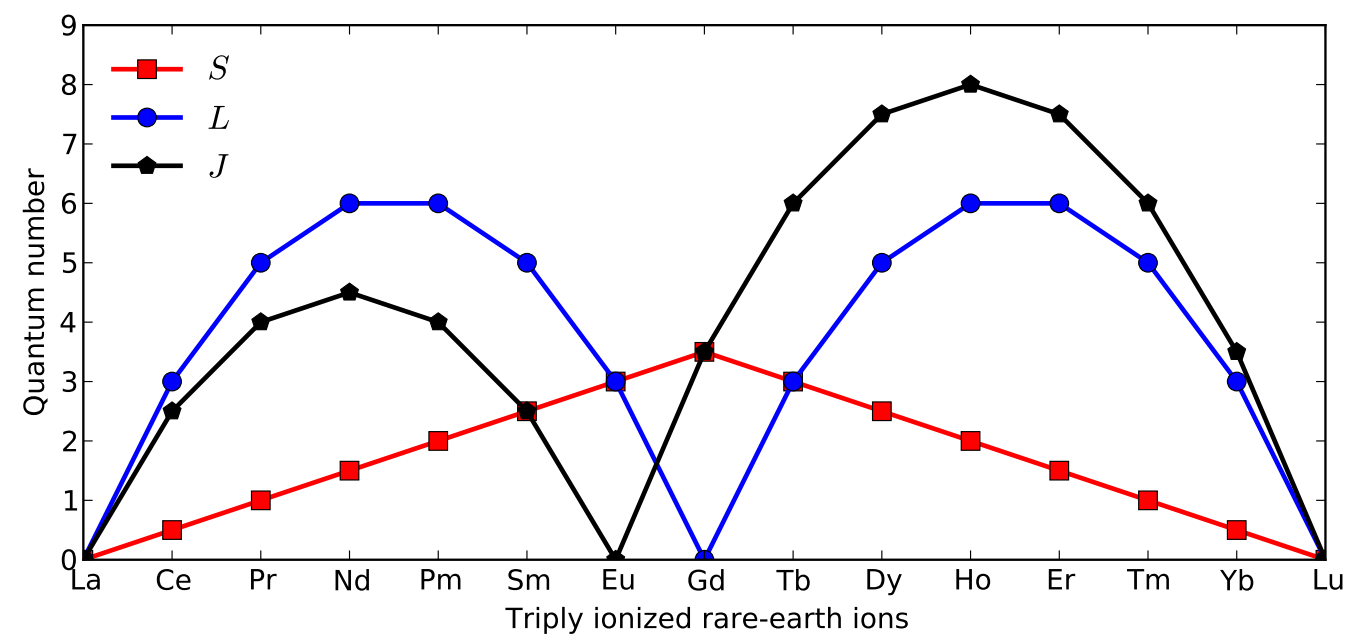

Figure 2.2: Hund's rule ground state values of $S, L$, and $J$ for the triply ionized rare-earth ions.

${ }^{6} \mathrm{H}_{5 / 2} \cdot{ }^{3}$ The Hund's rules ground state values of $J, L$ and $S$ for the trivalent rare-earth ions are shown in Figure 2.2. We note that in the first half of the series, where $J=|L-S|, L$ exceeds both $S$ and $J$ in some cases, which gives rise to orbital dominant magnetism.

In the following sections, we discuss how the Hund's rule ground state values of $J, L$ and $S$, determine the magnetic properties of rare-earth ions.

\subsection{Rare-earth Magnetism}

In this section we discuss rare-earth magnetism, which will provide crucial background for later sections. In general rare-earth based materials have quite different magnetic properties compared to itinerant $d$ electron systems. This comes primarily from the fact that the $4 f$ wave functions are spatially localized and atomic-like, which has some important consequences:

- The spin-orbit coupling of the $4 f$ electrons is large compared to crystal field and Zeeman energies, so that usually only the ground-state $J$ multiplet is important.

\footnotetext{
${ }^{3}$ In this thesis we do not use the term symbols, but instead write out $J, L, S$ explicitly.
} 
- The orbital moments are not necessarily quenched, ${ }^{4}$ as is often the case in light transition metal systems.

- The magnetic moments reside mainly in the localized $4 f$ shell, with only small contributions from band magnetism.

- The inter-ion exchange producing ferromagnetism cannot arise from direct exchange between $4 f$ orbitals, as the inter-ionic $4 f$ wave functions do not appreciably overlap.

- Carrier mediated exchange mechanisms typical of rare-earth metals are not as important in the rare-earth nitrides, which are semiconductors.

These features will play a key role in later Chapters. Now we proceed to outline in more detail the basic magnetic features of the rare-earth ions, including more in-depth discussion of the above points.

\subsubsection{Atomic magnetism}

We start by examining a quantum mechanical model of atomic magnetism. We define $\mathbf{H}$ with magnitude $|\mathbf{H}|=H$, as the uniform applied magnetic field. ${ }^{5}$ The effect of $\mathbf{H}$ on a single electron is twofold. First, the momentum of the electron is modified by $\mathbf{p}_{i} \rightarrow \mathbf{p}_{i}+\frac{|e|}{c} \mathbf{A}_{i}$ where $\mathbf{A}_{i}$ is the vector potential associated with $\mathbf{H}$. Second, the interaction of the electron spin $\mathbf{s}_{i}$ with $\mathbf{H}$ must be accounted for by adding $\frac{|e| \hbar}{m c} \mathbf{H} \cdot \mathbf{s}_{i}$ to the Hamiltonian. ${ }^{6}$

This means that the Hamiltonian for the partially filled $4 f$ shell described previously, $\mathscr{H}_{e-e}+\mathscr{H}_{S O}$, then acquires additional terms. By choosing the vector potential as $\mathbf{A}=\frac{1}{2} \mathbf{H} \times \mathbf{r}$, the inclusion of these two effects yields

$$
\mathscr{H}_{4 f}=\mathscr{H}_{4 f}^{0}+\frac{|e|}{2 m_{e} c} \mathbf{H} \cdot \sum_{i} \mathbf{r}_{i} \times \mathbf{p}_{i}+\frac{e^{2}}{8 m_{e} c^{2}} \sum_{i}\left(\mathbf{H} \times \mathbf{r}_{i}\right)^{2}+\frac{|e| \hbar}{m_{e} c} \mathbf{H} \cdot \mathbf{S},
$$

\footnotetext{
${ }^{4}$ Quenching of the orbital moment occurs when $\left\langle L_{z}\right\rangle=0$

${ }^{5}$ We use the cgs unit Oe for $\mathrm{H}$, though in some chapters $\mu_{0} H$ which has units of Tesla is used.

${ }^{6}$ This can be derived by an expansion of the Dirac equation [24, 25].
} 


\section{Chapter 2 Theoretical Background}

where $\mathscr{H}_{4 f}^{0}=\mathscr{H}_{e-e}+\mathscr{H}_{S O}$ is the Hamiltonian in the absence of $\mathbf{H}$. We can simplify this expression by using the definition of the total orbital momentum operator $\hbar \mathbf{L}=\sum_{i} \mathbf{r}_{i} \times \mathbf{p}_{i}$, to replace the second term above, and define the Bohr magneton as $\mu_{B}=|e| \hbar / m_{e} c$ :

$$
\mathscr{H}_{4 f}=\mathscr{H}_{4 f}^{0}+\mu_{B}(\mathbf{L}+2 \mathbf{S}) \cdot \mathbf{H}+\frac{e^{2}}{8 m_{e} c^{2}} \sum_{i}\left(\mathbf{H} \times \mathbf{r}_{i}\right)^{2}
$$

The last term is responsible for diamagnetism, and is much smaller compared to the first term, and so we may ignore it in the following discussion [23]. The term linear in $\mathbf{H}$ is known as the Zeeman term, and the prefactor of $\mathbf{H}$ is the magnetic moment operator, ${ }^{7}$ which we denote $\mathbf{m}=-\mu_{B}(\mathbf{L}+2 \mathbf{S})$. The expectation value of $\mathbf{m}$ is what is measured experimentally, and with the choice of $\mathbf{H}$ in the $z$-direction, the magnetic moment is given by

$$
m=-\mu_{B}\left\langle L_{z}+2 S_{z}\right\rangle . \quad \text { magnetic moment }
$$

Often we will speak of the spin and orbital contribution to the total moment, given by

$$
\begin{array}{lc}
m_{L}=-\mu_{B}\left\langle L_{z}\right\rangle & \text { orbital moment } \\
m_{S}=-2 \mu_{B}\left\langle S_{z}\right\rangle & \text { spin moment. }
\end{array}
$$

The magnetic moment is present even in the absence of a field, and is only non-zero for partially filled shells.

We haven't precisely defined the meaning of the expectation values $\langle\cdots\rangle$ used above. In general, to calculate the expectation value of $\mathbf{m}$ at a temperature $T$, we must use an ensemble or "thermodynamic" average. For an arbitrary quantum mechanical operator $\mathcal{O}$, the thermodynamic average is given by

$$
\langle\mathcal{O}\rangle_{T}=\frac{1}{\mathcal{Z}} \sum_{n}\langle n|\mathcal{O}| n\rangle e^{-\beta E_{n}}
$$

\footnotetext{
${ }^{7}$ This is analogous to the classical magnetic dipole moment, with energy $E=-\mathbf{m} \cdot \mathbf{H}$
} 
where $\beta=\left(k_{B} T\right)^{-1}$ with $k_{B}$ Boltzmann's constant. The $|n\rangle$ are eigenstates of $\mathscr{H}$ with associated energy $E_{n}: \mathscr{H}|n\rangle=E_{n}|n\rangle$. The partition function $\mathcal{Z}$ is given by

$$
\mathcal{Z}=\sum_{n} e^{-\beta E_{n}}
$$

If the system is in its ground state at $T=0$, then $\langle\mathcal{O}\rangle_{T}=\langle 0|\mathcal{O}| 0\rangle$ where $|0\rangle$ is the ground state. In this thesis, $\langle\cdots\rangle$ should be taken as the ground state expectation value if the states are not explicitly indicated.

As we shall later see, the magnetic moment can be calculated for the free-ion case of the rare-earth elements relatively easily. In a crystalline environment however, the presence of neighboring ions makes calculation of the magnetic moment and other related quantities more complex.

We next turn to the magnetic properties of a collection of $N$ ions in the presence of a magnetic field.

\subsubsection{Paramagnetism}

We follow a general approach for discussing paramagnetism, as outlined in Nolting \& Ramakanth [22]. The aim is to gain a good understanding of how the strong spin-orbit coupling of the rare-earths, in combination with the Zeeman coupling, influences the paramagnetic response. We start by considering a system of $N$ identical, non-interacting ions in a magnetic field $\mathbf{H}$. In this case, the Hamiltonian is just the sum of the effective Hamiltonian for a single ion:

$$
\mathscr{H}=\sum_{i=1}^{N} \mathscr{H}_{e-e}^{i}+\mathscr{H}_{S O}^{i}+\mathscr{H}_{Z}^{i},
$$

where the sum is over $N$ ions and $\mathscr{H}_{Z}=-\mathbf{m} \cdot \mathbf{H}$ is the Zeeman term. The average magnetic moment of this system is called the magnetization $\mathbf{M}$, related to the magnetic moment of a single ion by

$$
\mathbf{M}=\frac{N}{V}\langle\mathbf{m}\rangle,
$$




\section{Chapter 2 Theoretical Background}

where $V$ is the volume of the system. We will calculate $\mathbf{M}$ for a rare-earth ion, but first it helps to make the connection to thermodynamics. For a Hamiltonian $\mathscr{H}$ which has eigenvalues $E_{n}$, the free energy $F$ is defined as $e^{-\beta F}=\sum_{n} e^{-\beta E_{n}}=\mathcal{Z}$, or equivalently $F=-\frac{1}{\beta} \ln \mathcal{Z}$, where $\mathcal{Z}$ is the partition function. The magnetization can then be written

$$
\begin{aligned}
\mathbf{M}(T, H) & =-\left.\frac{1}{V} \frac{\partial F}{\partial \mathbf{H}}\right|_{T} \\
& =\frac{1}{\beta V}\left(\frac{\partial}{\partial \mathbf{H}} \ln \mathcal{Z}\right)_{T} .
\end{aligned}
$$

We can also define the volume susceptibility $\chi$, which links the response of the system (the magnetization) to the applied magnetic field. In general it is a tensor,

$$
\begin{aligned}
\chi_{\alpha \beta} & =\frac{\partial M_{\alpha}}{\partial H_{\beta}} \\
& =-\frac{1}{V}\left(\frac{\partial^{2} F}{\partial H_{\alpha} \partial H_{\beta}}\right)_{T},
\end{aligned}
$$

where $\alpha, \beta=x, y, z$ label the Cartesian components. In this thesis we are usually mostly interested in one component and take $H$ in the $z$ direction, so we do not use explicit subscripts for the components. Thus $\mathbf{M}=(0,0, M)$, and $\chi(T)=\partial M / \partial H$, without explicit subscript.

To calculate the magnetization, we note that the partition function of $N$ ions factorizes as $\mathcal{Z}=\left(\mathcal{Z}_{0}\right)^{N}$, where $\mathcal{Z}_{0}$ is the single-ion partition function

$$
\mathcal{Z}_{0}=\sum_{n}\left\langle n\left|e^{-\beta\left(\mathscr{H}_{e-e}+\mathscr{H}_{S O}+\mathscr{H}_{Z}\right)}\right| n\right\rangle
$$

The magnetization can then be calculated by

$$
M=-\frac{N}{V} k_{B} T \frac{\partial \ln \mathcal{Z}_{0}}{\partial H}
$$

To calculate $\mathcal{Z}_{0}$ we must define the states $\{|n\rangle\}$. In $L S$ coupling, the basis states are given by $\left\{\left|L S J M_{J}\right\rangle\right\}$, but when a magnetic field is present another basis labeled by $\left\{\left|L S M_{S} M_{L}\right\rangle\right\}$ is sometimes convenient. As we saw 
earlier, $\mathscr{H}_{e-e}$ does not depend on $J, L$ or $S$, and just sets an energy scale: $\left\langle n\left|\mathscr{H}_{e-e}\right| n\right\rangle=E_{0}$. The choice of basis now depends on whether the spin-orbit term or Zeeman term is larger. In the rare-earths, the spin-orbit term is always larger than the Zeeman term for typical laboratory magnetic fields, and the resulting magnetization is sometimes referred to as the "weak-field Zeeman effect". Recall that in $L S$-coupling the spin-orbit term then takes the form

$$
\mathscr{H}_{S O}=\Lambda \mathbf{L} \cdot \mathbf{S}
$$

however this is not diagonal in the basis $\left\{\left|L S M_{S} M_{L}\right\rangle\right\}$, but only in the basis $\left\{\left|J M_{J} L S\right\rangle\right\} \equiv\left\{\left|J M_{J}\right\rangle\right\}{ }^{8}$ This can be seen by noting $\mathbf{L} \cdot \mathbf{S}=\frac{1}{2}\left(\mathbf{J}^{2}-\mathbf{L}^{2}-\mathbf{S}^{2}\right)$, which has the eigenvalue of $\frac{1}{2}[J(J+1)-L(L+1)-S(S+1)]$ when acting on $\left|J M_{J}\right\rangle$. The expectation value of $\mathscr{H}_{S O}$ is then

$$
\left\langle J M_{J}\left|\mathscr{H}_{S O}\right| J M_{J}\right\rangle=\frac{1}{2} \Lambda(J(J+1)-L(L+1)-S(S+1)) .
$$

We now determine the Zeeman term in this basis. If the field is in the $z$ direction the Zeeman term takes the form

$$
\mathscr{H}_{Z}=-\mathbf{m} \cdot \mathbf{H}=\mu_{B}\left(L_{z}+2 S_{z}\right) H=\mu_{B}\left(J_{z}+S_{z}\right) H
$$

However, $\mathscr{H}_{Z}$ is not diagonal in the $J M_{J}$ basis because of the presence of $S_{z}$, so $\mathscr{H}_{S O}$ and $\mathscr{H}_{Z}$ do not share the same eigenstates. We note that $\left[S_{z}, J_{z}\right]=0$, so if we are restricted to only the ground state $J$-multiplet, $\mathscr{H}_{Z}$ is diagonal with respect to $M_{J}$ within this $J$-multiplet. Only with inclusions of higher $J$ multiplets does $S_{z}$ cause off-diagonal terms.

The expectation value of $\mathscr{H}_{Z}$ within the ground state $J$-multiplet can be calculated with the help of the Wigner-Eckart Theorem [23], ${ }^{9}$ which states that the matrix elements of any vector operator in the $2 J+1$ dimensional space of

\footnotetext{
${ }^{8}$ We refer to $\left\{\left|J M_{J} L S\right\rangle\right\} \equiv\left\{\left|J M_{J}\right\rangle\right\}$ as the $J M_{J}$ basis because $L$ and $S$ are fixed, depending only on the rare-earth ion, while all other quantum numbers $J, M_{J}, M_{L}, M_{S}$ are not necessarily fixed.

${ }^{9}$ See Appendix A for a more general discussion of the Wigner-Eckart theorem.
} 


\section{Chapter 2 Theoretical Background}

$\mathbf{J}$ and $J_{z}$ is proportional to the matrix elements of $\mathbf{J}$, thus we can write

$$
\left\langle J M_{J}\left|J_{z}+S_{z}\right| J M_{J}\right\rangle=g_{J}\left\langle J M_{J}\left|J_{z}\right| J M_{J}\right\rangle=g_{J} M_{J}
$$

where $g_{J}$ is the constant of proportionality known as the Landé g-factor:

$$
g_{J}=\frac{3}{2}+\frac{1}{2}\left[\frac{S(S+1)-L(L+1)}{J(J+1)}\right] .
$$

The Zeeman energy is thus given by $\left\langle J M_{J}\left|\mathscr{H}_{Z}\right| J M_{J}\right\rangle=g_{J} M_{J} \mu_{B} H$. We can now show that in the $J M_{J}$ basis the Hamiltonian takes the expectation value

$$
\langle\mathscr{H}\rangle=\underbrace{E_{0}+\frac{1}{2} \Lambda[J(J+1)-L(L+1)-S(S+1)]}_{E_{L S J}^{0}}+g_{J} M_{J} \mu_{B} H .
$$

Figure 2.3 shows a sketch of the energy levels for $\mathrm{SmN}$ where $\Lambda \approx 430 \mathrm{~K},{ }^{10}$ and so the separation between the $J=5 / 2$ ground state and $J=7 / 2$ state is about $1500 \mathrm{~K} . \mathrm{Sm}^{3+}$ has the second smallest separation between ground and the first excited multiplet among the rare-earths after $\mathrm{Eu}^{3+}$, which has a separation $500 \mathrm{~K}$, while for $\mathrm{Nd}^{3+}$ the separation increases to $2750 \mathrm{~K}$ and for $\mathrm{Yb}^{3+}$ it goes to $14,800 \mathrm{~K}[26]$.

The partition function can now be written as

$$
\mathcal{Z}_{0}=\sum_{J=|L-S|}^{J=|L+S|}\left(e^{-\beta E_{L S J}^{0}} \sum_{M_{J}=-J}^{J} e^{-\beta g_{J} M_{J} \mu_{B} H}\right)
$$

Because $\Lambda$ is large in the rare-earths compared to the temperatures of interest (less than $300 \mathrm{~K}$ ), we only need to consider the Hund's ground state value of $J$, which simplifies $\mathcal{Z}_{0}$ to

$$
\mathcal{Z}_{0}=e^{-\beta E_{L S J}^{0}} \sum_{M_{J}=-J}^{J} e^{-\beta g_{J} M_{J} \mu_{B} H}
$$

\footnotetext{
${ }^{10}$ We frequently use Kelvins to denote energies, however Boltzmann's constant $k_{B}$ must be included to convert to an actual energy unit, e.g. $k_{B} 300 \mathrm{~K} \approx 25 \mathrm{meV}$.
} 


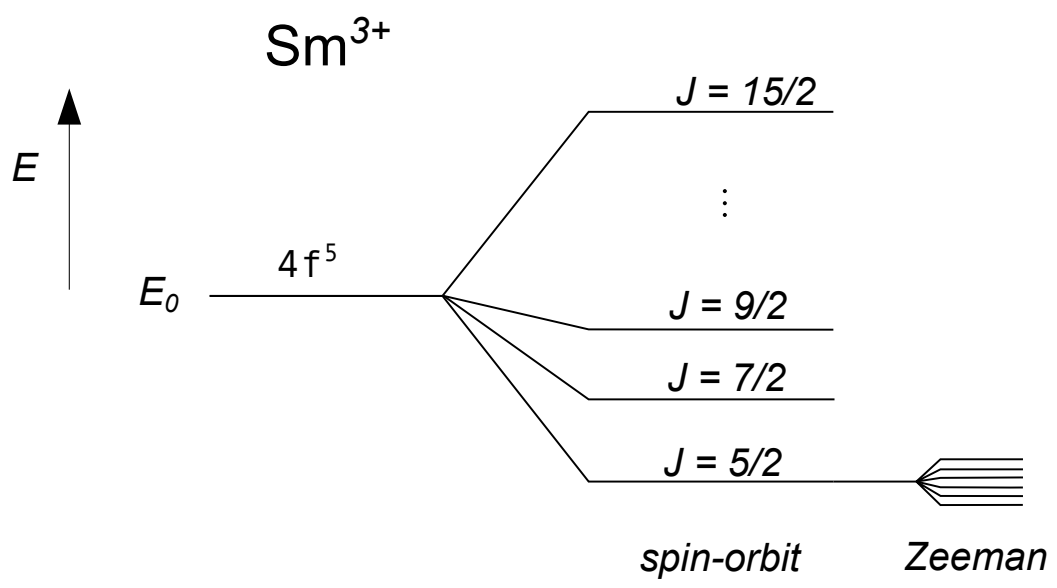

Figure 2.3: The energy levels of $\mathrm{Sm}^{3+}\left(4 f^{5}\right)$ free-ion, where the electronelectron interaction and all other atomic terms have been incorporated into $E_{0}$. The spin orbit term orders states by $J$ values, with separation $E_{J+1}-E_{J}=$ $\Lambda(J+1)$. The Zeeman term breaks the degeneracy among $M_{J}$ values. The higher $J$-multiplets are also split by the Zeeman term, though not shown here.

The sum can be written in terms of hyperbolic functions,

$$
\mathcal{Z}_{0}=e^{-\beta E_{L S J}^{0}} \frac{\sinh \left(\beta g_{J} \mu_{B} H\left(J+\frac{1}{2}\right)\right)}{\sinh \left(\frac{1}{2} \beta g_{J} \mu_{B} H\right)},
$$

and by calculating the magnetization as $M=-\frac{N}{V} k_{B} T \frac{\partial \ln \mathcal{Z}_{0}}{\partial H}$, we find

$$
M=\frac{N}{V} J g_{J} \mu_{B} B{ }_{J}\left(\beta g_{J} J \mu_{B} H\right)
$$

where $B_{J}$ is the Brillouin function [23]. When the field $H$ becomes large, $B_{J}(x) \rightarrow 1$ and the magnetization is the saturation magnetization

$$
M=\frac{N}{V} J g_{J} \mu_{B}
$$

here the susceptibility $\partial M / \partial H$ is zero. This corresponds to the saturation moment of $g_{J} J \mu_{B}$ for a single ion. We can also calculate the susceptibility in the high temperature limit where $k_{B} T \gg \mu_{B} H$. Then $B_{J}(x) \rightarrow \frac{J+1}{3 J} x$ and 
$\chi=\partial M / \partial H$ gives us Curie's law for a free ion:

$$
\chi=\frac{N}{V} \mu_{B}^{2} \frac{g_{J}^{2} J(J+1)}{3 k_{B} T} .
$$

Often this will be written in terms of the effective paramagnetic moment $m_{\text {eff }}=g_{J} \sqrt{J(J+1)} \mu_{B}$. As we shall see Equation (2.31) can be modified in a solid due to crystal field effects, in which case $m_{\text {eff }}$ takes on a different value from the free-ion value.

We emphasize that this form of Curie's law was derived on the basis that the spin-orbit coupling is larger than both the Zeeman splitting $\left(\Lambda \gg \mu_{B} H\right)$ and the thermal energy $\left(\Lambda \gg k_{B} T\right)$, while the thermal energy is larger than the Zeeman splitting $\left(k_{B} T \gg \mu_{B} H\right)$.

\subsubsection{Van Vleck paramagnetism}

A subtle correction to Curie's law occurs if at a given temperature, the spacing between $J$-multiplets is not very large $\left(\Lambda \gtrsim k_{B} T\right)$. In the rare-earths, $\mathrm{Eu}^{3+}$ and $\mathrm{Sm}^{3+}$ are most strongly affected by this due to their small multiplet separations. The $\mathrm{Eu}^{3+}$ ion, with $4 f^{6}$, has a $J=0$ ground state which is nonmagnetic. However, the van Vleck contribution allows for paramagnetism due to the presence of higher multiplets. In $\mathrm{Sm}^{3+}$, the van Vleck contribution is not as large, but is still appreciable.

The derivation of the van Vleck term comes from the consideration of higher $J$-multiplets in the partition function, thus one has to calculate

$$
\mathcal{Z}_{0}=e^{-\beta E_{L S}^{0}} \sum_{J=|L-S|}^{|L+S|} \sum_{M_{J}=-J}^{J} e^{-\beta\left(\mathscr{H}_{S O}+\mathscr{H}_{Z}\right)} .
$$

The calculation is shown in Nolting \& Ramakanth [22]. For the light rareearths where $J=|L-S|$, and if only the first excited $J$ multiplet contributes, the resulting van Vleck susceptibility is

$$
\chi_{V V}=\frac{N}{V}\left(\frac{J(J+1)}{3 k_{B} T} g_{J}^{2} \mu_{B}^{2}+\mu_{B}^{2} \frac{2(L+1) S}{3(J+1) \Delta}\right),
$$


where $\Delta=E_{J+1}-E_{J}$. The origin of this correction is due to the operator $S_{z}$ in $\mathscr{H}_{Z}$, which is non-diagonal in the $J M_{J}$ basis. Because it is not diagonal, it creates transitions between $J$-multiplets, which manifest in the temperatureindependent term. This term is present even when only the ground state multiplet is thermally occupied. For $\mathrm{Sm}^{3+}$ the ratio of the van Vleck term to the Curie term is

$$
\frac{\chi_{V V}}{\chi_{C}}=12 \frac{k_{B} T}{\Delta}=0.008 T \mathrm{~K}^{-1}
$$

where $\Delta=1500 \mathrm{~K}$. Thus at $125 \mathrm{~K}$ the two contributions have equal magnitude, with being $\chi_{V V}$ dominant above $125 \mathrm{~K}$. While this situation is altered to some degree in a solid due to the effects of exchange and crystal field interactions, the van Vleck contribution is still significant. This indicates that the $J$ mixing from the excited $J=7 / 2$ should be taken into account in any quantitative calculation involving the magnetic moment in trivalent Sm systems like SmN.

\subsubsection{Ferromagnetism}

Ferromagnetism arises due to the exchange interaction between ions which tends to align the spins. This can be understood from the Pauli exclusion principle, which states that a multi-electron wave-function must be antisymmetric with respect to switching any two coordinates. For a two electron system this means $\psi\left(\mathbf{r}_{1} \sigma_{1}, \mathbf{r}_{2} \sigma_{2}\right)=-\psi\left(\mathbf{r}_{2} \sigma_{2}, \mathbf{r}_{1} \sigma_{1}\right)$ where $\sigma_{i}$ is the spin of the $i$-th electron. For an $N$-electron system the multi-electron wave function $\Psi\left(\mathbf{r}_{1} \sigma_{1}, \ldots, \mathbf{r}_{N} \sigma_{N}\right)$ can in general be written as a sum of Slater determinants to satisfy the Pauli principle [23]. In the Hartree-Fock approximation, the many body state is taken as single Slater determinant, and we use this approximation here. If we calculate the expectation value of electron-electron interaction term $\mathscr{H}_{e-e}$ using $\Psi\left(\mathbf{r}_{1} \sigma_{1}, \ldots, \mathbf{r}_{N} \sigma_{N}\right)$, we find

$$
\left\langle\Psi\left|\mathscr{H}_{e-e}\right| \Psi\right\rangle=\frac{1}{2} \sum_{i, j}\left(U_{i j}-\delta_{\sigma_{i}, \sigma_{j}} J_{i j}\right)
$$




\section{Chapter 2 Theoretical Background}

where the sums are over the occupied electronic states and $\delta_{\sigma_{i}, \sigma_{j}}$ is the delta function between spins. The "direct" term $U_{i j}$ is given by

$$
U_{i j}=\int d \mathbf{r} d \mathbf{r}^{\prime}\left|\psi_{i}(\mathbf{r})\right|^{2} \frac{e^{2}}{\left|\mathbf{r}-\mathbf{r}^{\prime}\right|}\left|\psi_{j}\left(\mathbf{r}^{\prime}\right)\right|^{2}
$$

and the "exchange term" $J_{i j}$ is given by

$$
J_{i j}=\int d \mathbf{r} d \mathbf{r}^{\prime} \psi_{i}^{*}(\mathbf{r}) \psi_{j}^{*}\left(\mathbf{r}^{\prime}\right) \frac{e^{2}}{\left|\mathbf{r}-\mathbf{r}^{\prime}\right|} \psi_{i}\left(\mathbf{r}^{\prime}\right) \psi_{j}(\mathbf{r}),
$$

The direct term is the analogue to the classical Coulomb repulsion between electrons, independent of their spin. Due to the delta function, the exchange term is only present when two spins have the same value. Because the sign in front of $J_{i j}$ is negative in Eq. (2.35), this means that the two electrons with the same spin will have a lower energy. This results in a tendency for the spins to align themselves, ${ }^{11}$ and is the basis of ferromagnetism.

\section{Mean-field approximation}

Calculating the exchange integrals is extremely difficult in solids, so usually an effective theory is preferred. The Heisenberg Hamiltonian is the most well known effective theory of exchange, where the exchange integral is replaced with an exchange constant $J_{i, j}$, which might be obtained empirically. The Heisenberg Hamiltonian is given by

$$
\mathscr{H}=-\sum_{i \neq j} J_{i, j} \mathbf{S}_{i} \cdot \mathbf{S}_{j}
$$

where the sum is over all pairs of spins $\mathbf{S}_{i}$ and $\mathbf{S}_{j}(i, j=1, \ldots, N)$. We note that for parallel spins, the energy is at a minimum (assuming $J_{i, j}$ is positive, for ferromagnetic behavior). The Heisenberg model is an approximation best suited for localized moments, which is the case in the rare-earths with tightly bound $4 f$ shells. Furthermore, we assume the exchange $J_{i, j}$ is isotropic. The study of this Hamiltonian encompasses a vast body of literature, and here we

\footnotetext{
${ }^{11}$ While the Coulomb energy may be reduced, the kinetic energy may increase enough to make ferromagnetism unfavorable, depending on the band structure.
} 
review one of the basic results, known as the mean-field or molecular field approximation. ${ }^{12}$

In the mean-field approximation, the Heisenberg Hamiltonian can be rewritten $\operatorname{as}^{13}$

$$
\mathscr{H}_{\text {ex }}=-2 J_{\text {ex }} \sum_{i=1}^{N} S_{z}^{i}\left\langle S_{z}\right\rangle
$$

where fluctuations from the thermodynamic average have been ignored, and the quantization axis is taken along $z$. Translational symmetry is assumed (i.e. $\left\langle S_{z}^{i}\right\rangle \equiv\left\langle S_{z}\right\rangle$ ), and the sum is over $N$ spins. Equation (2.39) treats the exchange by assuming each spin $S_{z}^{i}$ feels an average exchange due to all the other spins in the system. When an applied field is present, the Zeeman term $g_{J} \mu_{B} H \sum_{i} J_{z}^{i}$ can be added. Note the presence of total angular momentum $J_{z}$, and not $S_{z}$ in the Zeeman term. ${ }^{14}$ It is more convenient to use $J_{z}$ in the exchange term, so we make use of the Wigner Eckart theorem, and note that within a single multiplet $S_{z}=\left(g_{J}-1\right) J_{z}$, so we can write the Hamiltonian including the Zeeman term as

$$
\mathscr{H}=g_{J} \mu_{B}\left(H-\frac{2 J_{e x}\left(g_{J}-1\right)^{2}}{g_{J} \mu_{B}}\left\langle J_{z}\right\rangle\right) \sum_{i} J_{z}^{i} .
$$

Written this way it is clear that the exchange acts as an effective magnetic field $H_{\text {eff }}$, given by

$$
H_{e f f}=-\frac{2 J_{e x}\left(g_{J}-1\right)^{2}}{g_{J} \mu_{B}}\left\langle J_{z}\right\rangle
$$

If we use the definition of the magnetization as $M=-\frac{N}{V} g_{J} \mu_{B}\left\langle J_{z}\right\rangle$, we link

\footnotetext{
${ }^{12}$ See for example Nolting \& Ramakanth [22].

${ }^{13}$ Only one component of $\mathbf{S}$ is non-zero within the approximation, chosen as the $z$ component

${ }^{14}$ It is often not emphasized in textbooks that in general, $S_{z} \neq J_{z}$ when discussing the exchange interaction. For the rare-earths, it is crucial to consider the orbital contribution.
} 
$H_{\text {eff }}$ and $M$ :

$$
H_{e f f}=\frac{2 J_{e x}\left(g_{J}-1\right)^{2}}{(N / V)\left(g_{J} \mu_{B}\right)^{2}} M=\lambda M .
$$

Thus the effect of exchange is to modify the applied field, with the modified field given by $\widetilde{H}=H+\lambda M=H(1+\lambda \chi)$. This also implies that the meanfield approximation must be self-consistent in the sense that magnetization is a function of itself. This can be seen by using the result for the magnetization (Eq. 2.29) found before, except now in terms of $\widetilde{H}$ :

$$
M(T)=\frac{N}{V} g_{J} \mu_{B} J B_{J}\left(\beta g_{J} \mu_{B}(H+\lambda M(T)) .\right.
$$

The susceptibility can be calculated directly from Eq. 2.43, but alternatively we note that

$$
\chi=\frac{\partial M}{\partial H}=(1+\lambda \chi) \chi_{0}
$$

where $\chi_{0}=\partial M / \partial \widetilde{H}=\partial M /\left.\partial H\right|_{\lambda=0}$ is the susceptibility in the absence of exchange. This can be written in the form [27]:

$$
\frac{1}{\chi}=\frac{1}{\chi_{0}}-\lambda \text {. }
$$

If we take $\chi_{0}$ as the Curie law susceptibility derived in the previous section, the susceptibility including exchange is

$$
\chi=\frac{C}{T-C \lambda} \quad \text { where } C=\frac{N}{V} \frac{g_{J}^{2} \mu_{B}^{2} J(J+1)}{3 k_{B}} .
$$

We note that when $T=C \lambda \equiv \theta_{p}$, the susceptibility diverges. This is the paramagnetic Curie temperature. Below $\theta_{p}$ the system is in the ferromagnetic phase where a spontaneous magnetization may occur even if $H=0$. Above $\theta_{p}$, in the paramagnetic phase, the magnetization is only non-zero when a field 
is applied. In this approximation $\theta_{p}$ is explicitly given by

$$
\theta_{p}=\frac{2 J_{e x}\left(g_{J}-1\right)^{2} J(J+1)}{3 k_{B}} .
$$

We note that this estimation of $\theta_{p}$ considers only the ground state $J$-multiplet, and assumed $T$ was large compared to $\mu_{B} H$. For this reason we call $\theta_{p}$ the high-temperature paramagnetic Curie temperature. In general this does not coincide with the actual ordering point, $T_{C}$, though often it is relatively close if the assumptions given in the derivation hold. A more common version of this relationship is usually given when there is no orbital moment: $\theta_{p}=$ $2 J_{e x} S(S+1) / 3 k_{B}$. This is appropriate for $\mathrm{Gd}^{3+}$, which has $L=0$, but is not in general applicable to the other rare-earths.

Equation (2.47) allows us to estimate the strength of the exchange interaction from the experimentally measured susceptibility, which we will make use of in later chapters. We note that the origin of the exchange in the rare-earth nitrides is not fully understood, ${ }^{15}$ though various models have been put forth $[2,28]$. A contribution to exchange from conduction electrons which plays an important role in rare-earth metals (the so-called Ruderman-Kittel-KasuyaYosida (RKKY) interaction [22]) is not as important in the non-metallic rareearth nitrides. This is the reason for the much lower Curie temperatures found in the RENs compared to metallic rare-earth systems [28]. For example metallic $\mathrm{SmZn}, \mathrm{SmCd}$, and $\mathrm{SmAl}_{2}$ have ordering temperatures of about 128, 195, and $125 \mathrm{~K}$, while SmN orders around $27 \mathrm{~K}$ [29, 30].

Due to the localized nature of the $4 f$ electrons the direct $4 f-4 f$ inter-ion exchange interaction is negligible, so the exchange interaction must proceed indirectly through superexchange mechanisms, which involve unoccupied $5 d$ states. It is commonly assumed that through intra-ion $4 f-5 d$ exchange, ferromagnetic exchange exists between nearest neighbor (nn) rare-earth ions via the $4 f-5 d-5 d-4 f$ chain. Another exchange channel is antiferromagnetic superexchange via the $\mathrm{N} 2 p$ states: $4 f-5 d-\mathrm{N} 2 p-5 d-4 f$ (next nearest neighbor, nnn). In this picture there are then two contributions to the overall exchange energy,

\footnotetext{
${ }^{15}$ We have only considered an effective exchange so far, ignoring the underlying states responsible.
} 


\section{Chapter 2 Theoretical Background}

and thus to the ordering temperature, with $T_{C} \sim\left(J_{n n}+J_{n n n}\right) / k_{B}$, where $J_{n n}$ is the ferromagnetic and $J_{n n n}$ the antiferromagnetic exchange. These contributions through $p$ and $d$ states mean the exchange is not isotropic, as we have assumed in the mean-field theory. Experimentally however, it is not always possible through magnetization measurements to distinguish different contributions to $T_{C}$ (or $\theta_{p}$ ), and one can only extract a single isotropic effective exchange constant, $J_{e x}$.

\subsubsection{The crystal field}

We have so far ignored the effect of the nitrogen ions on the magnetic properties of the RENs. In principle, it is possible to consider these effects within density functional theory techniques, though there have been very few of these studies on the rare-earth nitrides with the exception of GdN. Instead, an approach originally due to Bethe [31] can be used which treats the $\mathrm{N}$ anions as electric point charges. This so-called crystal field can then be incorporated as a potential energy term in the Hamiltonian of the magnetic ion.

For the rare-earths, the crystal field energy is smaller than the spin-orbit interaction because the $4 f$ orbital cloud is tightly bound, and shielded by the filled $5 s$ and $5 p$ shells, only weakly feeling the influence of the crystal field. The crystal field can then be considered as a perturbation to the spin-orbit coupling energy. The situation is reversed for light transition metal systems however, where the $d$ orbitals are delocalized enough to strongly feel the crystal field. In the transition metal case, the spin-orbit coupling acts as a perturbation on the crystal field Hamiltonian. For heavy transition metal systems and the actinides, where the $4 d, 5 d$ and $5 f$ shells are partially filled, the situation is somewhere in between, and the spin-orbit and crystal field energies lie on nearly equal footing.

One important consequence of the crystal field interaction is quenching of the orbital moment, where the expectation value of the orbital moment vanishes (i.e. $\left\langle L_{z}\right\rangle=0$ ). In this case the magnetic moment then resides only in the spin moment. Full quenching usually only occurs in systems where the spin-orbit coupling is small, such as the $3 d$ transition metals. 
One way to see this is to note that the crystal field potential is a real function of the coordinates (as we shall see), and if the ground state $\psi_{0}$ is non-degenerate, then $\psi_{0}$ may be chosen to be real; $\psi_{0}^{*}=\psi_{0}$. In this case, the expectation value of $L_{z}=-i \hbar \frac{\partial}{\partial \phi}$ can be calculated as

$$
\begin{aligned}
\left\langle\psi\left|L_{z}\right| \psi\right\rangle & =-i \hbar \int d \phi d(\cos \theta) \psi^{*}(\theta, \phi) \frac{\partial}{\partial \phi} \psi(\theta, \phi) \\
& =i \times \mathrm{constant}
\end{aligned}
$$

The result is thus an imaginary number for the expectation value $\left\langle L_{z}\right\rangle$, however all observable operators must have real eigenvalues. Thus $\left\langle L_{z}\right\rangle \equiv 0$ for real, non-degenerate wave functions, and the orbital angular momentum is said to be quenched. ${ }^{16}$ For a degenerate ground state, the orbital moment is not necessarily fully quenched, but will be partially quenched, depending on the symmetry properties of the degenerate states [33].

In contrast, the spin-orbit coupling tries to maximize the orbital moment given that a spin-moment is present. This is because the energy of the spinorbit term $\Lambda \mathbf{L} \cdot \mathbf{S}$ is minimized by having a large orbital moment, either parallel or antiparallel to $\mathbf{S}$, depending on the sign of $\Lambda$. The competition between $\mathscr{H}_{S O}$ and the crystal field term, $\mathscr{H}_{C F}$, then determines the degree of quenching. Thus the light $3 d$ systems, where $\left\langle\mathscr{H}_{S O}\right\rangle \ll\left\langle\mathscr{H}_{C F}\right\rangle$, are nearly fully quenched, while the rare-earths experience much less quenching. Any quenching that does occur in the rare-earths however, is also transferred to $\mathbf{S}$ due to the spin-orbit coupling. This is also the origin of the large magnetocrystalline anisotropy in rare-earths. We note that the effective paramagnetic moment of the light transition metals is often given by $m_{e f f}=g \mu_{B} \sqrt{S(S+1)}$ because the crystal field effectively sets $L=0$ [23], while for the rare-earths $J$ is still a good quantum number at large enough temperatures.

\footnotetext{
${ }^{16}$ This can also be shown to be a consequence of time-reversal invariance of the Hamiltonian, if the ground state is non-degenerate [32].
} 


\section{Chapter 2 Theoretical Background}

\section{Crystal field calculations}

While crystal field effects are generally smaller in the rare-earths than in transition metal systems, they are not negligible (except for the $\mathrm{Gd}^{3+}$ ion, with $L=0)$. Thus, in order to quantify the effects of the crystal field on $\mathrm{SmN}$ and NdN we carry out crystal field calculations in Chapter 4. Here we give an overview of the crystal field theory most applicable to rare-earth ions in cubic environments, which is outlined in detail by Hutchings when only the ground state $J$-multiplet is included [34]. A review by De Wijn et al. [35] extends the crystal field model for rare-earth ions to arbitrary multiplets; here we mostly follow their notation. Chapter 3 will describe further details for calculations within arbitrary multiplets, while here we present the basic theory.

The effect of the crystal field on a rare-earth ion can be written as an additional term in the rare-earth ion Hamiltonian corresponding to the electric potential energy;

$$
\mathscr{H}_{C F}=\sum_{i} \sum_{j} \frac{Z_{j} e^{2}}{\left|\mathbf{R}_{j}-\mathbf{r}_{i}\right|}
$$

where $-Z_{j}|e|$ is the ligand charge at position $\mathbf{R}_{j}$ ( $\mathrm{N}$ in the rare-earth nitride case) and $\mathbf{r}_{i}$ is the position of the $i$ th $4 f$ electron. Because the $4 f$ orbital radius, $\langle r\rangle_{4 f} \approx 0.5 \AA$ (approximately equal to the Bohr radius $\left.a_{B}=0.53 \AA\right)^{17}$, is smaller than the Re-N separation of about $2.5 \AA$, an expansion of the denominator is justified. Strictly speaking, this electrostatic approach is not fully justified (see discussion in, e.g. [36]), however for the ground state properties, the electrostatic approximation produces good agreement with experiment and is far more straightforward to apply compared to quantum-mechanical ab-inito techniques. This expansion is well known in electrostatics, and is usually carried out in either Cartesian coordinates, Legendre polynomials, or normalized spherical harmonics, $Y_{k}^{q}(\theta, \phi)$. Here, we carry out the expansion using tesseral harmonics $f_{k q}$, which are purely real functions, and are related to the spherical

\footnotetext{
${ }^{17}$ The Re-N separation is given by $a / 2$ where $a$ is the lattice constant. Experimental and theoretical lattice constants for the RENs are given in Ref. [2]
} 
harmonics by

$$
f_{k q}=\frac{r^{k}}{\sqrt{2} c_{k q}}\left[Y_{k}^{-q}+(-1)^{q} Y_{k}^{q}\right]
$$

where $c_{k q}$ are normalization constants. For example, $f_{40}$ is given by

$$
f_{40}(\mathbf{r})=35 z^{4}-30 z^{2} r^{2}+2 r^{2}
$$

Other tesseral harmonics are given in Ref. [34]. For a single point charge at position $\mathbf{R}_{j}$ and a single $4 f$ electron at $\mathbf{r}_{i}$ we can write the expansion as

$$
\frac{1}{\left|\mathbf{R}_{j}-\mathbf{r}_{i}\right|}=\sum_{k=0}^{\infty} \frac{1}{R_{j}^{2 k+1}}\left[\sum_{q=0}^{k} c_{k q}^{2} \frac{4 \pi}{2 k+1} f_{k q}\left(\mathbf{R}_{j}\right) f_{k q}\left(\mathbf{r}_{i}\right)\right]
$$

which is valid for $r_{i}<R_{j}$. The total contribution to $\mathscr{H}_{C F}$ is the sum of Eq. 2.53 over the $i 4 f$ electrons and $j$ N-ion point charges coordinating the rare-earth ion, leading to

$$
\mathscr{H}_{C F}=\sum_{k=0}^{6} \sum_{q=0}^{k} A_{k}^{q} \sum_{i} f_{k q}\left(\mathbf{r}_{i}\right)
$$

The sum over $k$ can be shown to be non-zero only for even values of $k \leqslant 6$ for the $4 f$ shell electrons, determined by the orthogonality properties of tesseral (or spherical) harmonics. The $A_{k}^{q}$ are constants describing the strength of the crystal field

$$
A_{k}^{q}=e^{2} \frac{4 \pi}{2 k+1} c_{k q}^{2} \sum_{j} Z_{j} \frac{f_{k q}\left(\mathbf{R}_{j}\right)}{R_{j}^{2 k+1}}
$$

where terms with $k=4,6$ describe the fourth and sixth order contributions to the crystal field. Often, instead of calculating the $A_{k}^{q}$ explicitly, it is easier to factor out the $q$ dependence by making use of the point symmetry of the ligand atoms, so that $A_{k}^{q}=A_{k} B_{q}$, where $B_{q}$ is a geometric scaling factor that takes into account all the $q$ dependence. We then use the $A_{k}$ to parametrize the crystal field strength. For cubic symmetry, as in the RENs, $\mathscr{H}_{C F}$ with the 


\section{Chapter 2 Theoretical Background}

$z$ axis along the [001] direction is given by

$$
\mathscr{H}_{\mathrm{CF}}=A_{4} \sum_{i}\left[f_{40}\left(\mathbf{r}_{i}\right)+5 f_{44}\left(\mathbf{r}_{i}\right)\right]+A_{6} \sum_{i}\left[f_{60}\left(\mathbf{r}_{i}\right)-21 f_{64}\left(\mathbf{r}_{i}\right)\right] .
$$

Only fourth and sixth order contributions are present in cubic symmetry. The crystal field parameters can be calculated in the point-charge model for octahedral coordination (six nearest Re neighbors) of the rare-earth ion as

$$
A_{4}=\frac{7}{16} \frac{Z e^{2}}{R^{5}}, \quad A_{6}=\frac{3}{64} \frac{Z e^{2}}{R^{7}}
$$

where $R$ is the Re- $\mathrm{N}$ distance. These parameters are depend on the coordination number of the magnetic ion, which can be 4,6, or 8 in cubic symmetry.

As we will see in Chapter 3 , the excited $J$-multiplets are important to consider, because, depending on the size of the crystal field parameters, offdiagonal matrix elements of $\mathscr{H}_{C F}$ can cause significant mixing between $J$ multiplets. This is especially important for $\mathrm{SmN}$, and to a lesser degree for NdN. In addition to the crystal field, the spin-orbit coupling and exchange may be considered, and so the Hamiltonian of a rare-earth ion is given by

$$
\mathscr{H}=\Lambda \mathbf{L} \cdot \mathbf{S}-2 J_{e x}\left\langle S_{z}\right\rangle S_{z}+\mathscr{H}_{C F},
$$

where the exchange is included via self-consistent mean-field theory. The exchange constant $J_{e x}$ is estimated from the paramagnetic susceptibility, as discussed earlier. This Hamiltonian will allow us to calculate the ground state magnetic moment as well as the temperature dependent susceptibility.

We remark that when only the ground state multiplet is considered, the effect of a cubic crystal field on the rare-earth ions is to remove some the degeneracy present in the $M_{J}$ levels in the absence of a field. This is sketched in Figure 2.4 for the $\mathrm{Sm}^{3+}$ ion, where the $J=5 / 2$ ground state (with $2 J+1=6$ degenerate levels) is split into a doubly degenerate state (doublet) labelled $\Gamma_{7}$ and a quadruply degenerate state (quartet) labelled $\Gamma_{8}$. The number and degeneracy of these crystal field states can be predicted on the basis of group theory [31, 37] with knowledge of the symmetry of the crystal field and the $J$ 


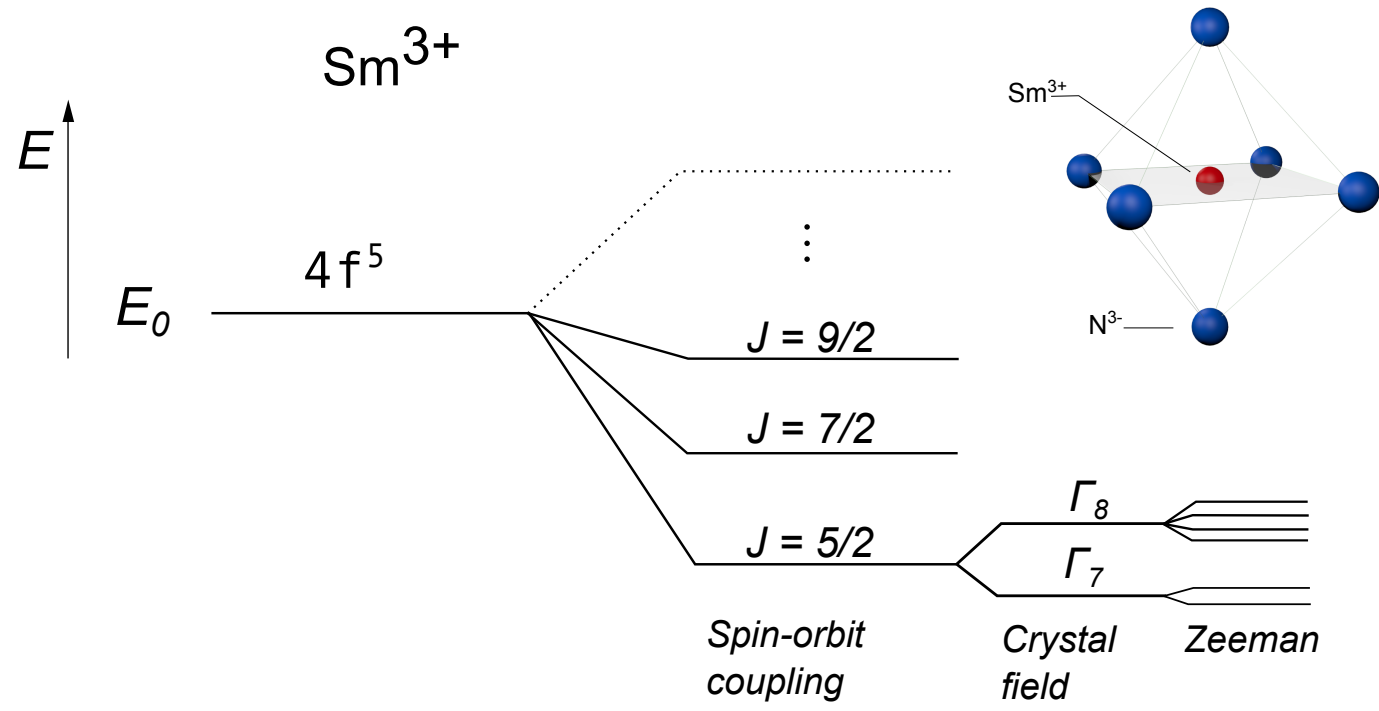

Figure 2.4: A sketch of the energy levels of $\mathrm{Sm}^{3+}$ in a cubic crystal field with octahedral coordination. The crystal field splits the ground state $J=5 / 2$ multiplet into a $\Gamma_{7}$ doublet and a $\Gamma_{8}$ quartet. Not shown are the crystal field and Zeeman splittings of the higher $J$-multiplets.

value of the ion. When an exchange or Zeeman term is present, these crystal field levels split again, though these splittings are small compared to the crystal field levels.

\subsection{Exchange Springs}

In this section we include a discussion of conventional exchange-spring systems, which are related to the topics discussed in Chapters 4 and 5. The first exchange-spring system was reported by by Goto et al. [38], who studied the magnetization of bilayer thin films composed of hard (large anisotropy) and soft (small anisotropy) ferromagnets. The study found that with a large field $\mathbf{H}_{0}$ applied in-plane, both layers were magnetized in the $x$-direction. When a small field $\mathbf{H}_{1}$ was subsequently applied in the $-x$ direction, a rotation of the soft layer magnetization formed for a large enough value of $\left|\mathbf{H}_{1}\right|$ (see Figure $2.5)$.

This was shown to be due to competing interactions within the soft ferromagnetic layer. Because the hard ferromagnetic is layer insensitive to the 


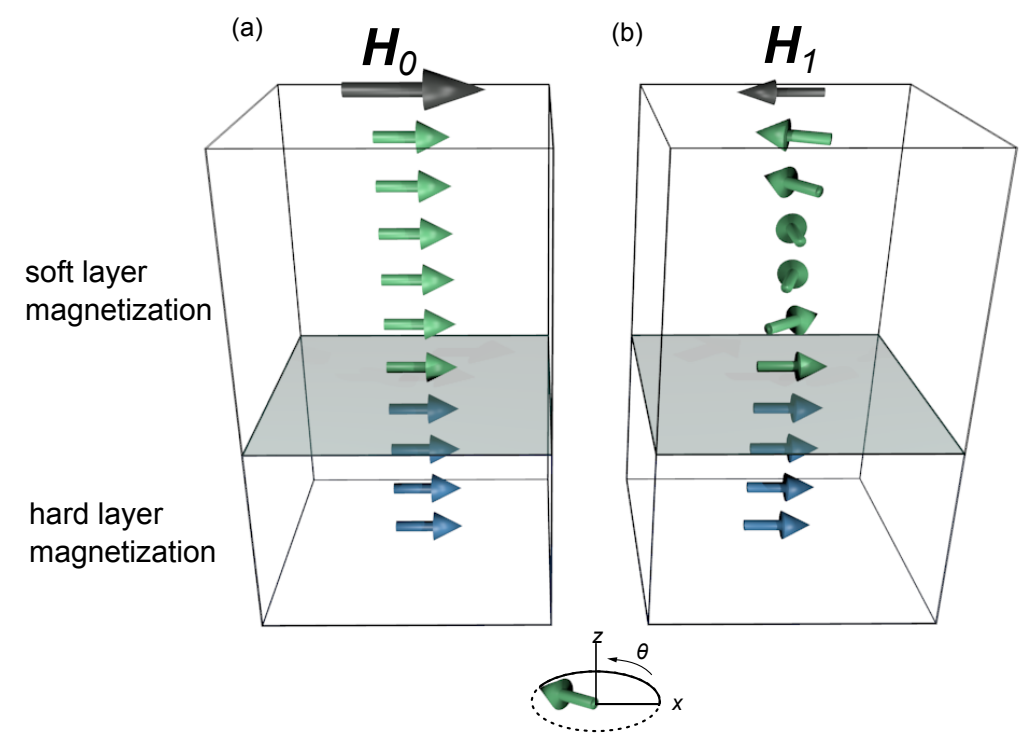

Figure 2.5: A sketch of a conventional exchange-spring system. (a) Both layers are magnetized in the $x$-direction with a large field $\mathbf{H}_{0}$. (b) A small field $\mathbf{H}_{1}$ begins to reverse the soft layer magnetization, while the hard layer remains fixed and pins the soft-layer magnetization at the interface, with an exchange spring forming in the soft layer.

smaller field in the $-x$ direction, it acted as a rigid layer which pinned the soft layer magnetization at the interface through ferromagnetic exchange coupling. Away from this interface, the Zeeman coupling of the soft layer caused the magnetic moment of ions to align with the field. The exchange interaction with the soft layer then mediated these two competing interactions, resulting in a gradual rotation of the spins. The term exchange-spring is used because of the analogy between a mechanical system made in the original paper, where the exchange coupling between the films is viewed as a spring.

The basic physics can be understood by treating the spins in a one dimensional model where each atomic plane in the soft layer rotates uniformly, while the hard layer is fixed. In this case, the magnetic free energy can be described in a micromagnetic approach [39].

Using the geometry of Figure 2.5, we can calculate the free energy per unit area of the soft layer, integrated of the thickness $L$ of the layer. In the simplest 
case, where various anisotropies can be ignored, the free energy is given by

$$
\mathcal{E}=\int_{0}^{L} \mathrm{~d} z\left[A\left(\frac{\mathrm{d} \theta(z)}{\mathrm{d} z}\right)^{2}-\mathbf{M} \cdot \mathbf{H}\right]
$$

where $A$ is a constant describing the strength of the exchange, and $\theta(z)$ is the azimuthal angle as shown in Fig. 2.5. The first term represents the energy cost associated with the magnetization of neighboring spins deviating from parallel. It can be derived by noting that the exchange energy between spins is $E_{e x} \sim$ $J_{e x} \cos \Delta \theta$, where $\Delta \theta$ is the angle between spins. This can be approximated for small angular displacements as $\Delta \theta \approx a \frac{\mathrm{d} \theta}{\mathrm{d} z}$ where $a$ is the separation between spins, and $z$ is the position in the film depth. Expanding the cosine leads to Equation 2.59 with $A=2 J_{e x} S^{2} / a^{3}$, where $S$ is the spin. The second term is just the Zeeman term, which tries to align the spins with the field.

The form of $\theta(z)$ which minimizes the free energy can be found in this case analytically using the calculus of variations, as was calculated by Goto. It gives the magnetization as $\mathbf{M}(z)=M(\cos \theta(z), \sin \theta(z), 0)$, and results in the Blochwall like behavior shown in Figure 2.5. We note that the two requirements for this model are are 1) a hard layer magnetization which is rigidly fixed, and 2) the magnetic field must reverse from its original direction, such that there is competition between interface exchange and the Zeeman coupling.

This domain-wall like magnetization phase is part of a more general set of competing interactions in magnetic systems. These "twisted phases" as we call them here, can be due to a variety of interactions. Interface exchange, bulk and surface anisotropies, and anisotropic or chiral exchange interactions can lead to diverse types of twisted phases. But all of these twisted magnetic observed so far have been in "conventional" spin-dominant, metallic systems. The light rare-earth nitrides provide the ability to host novel twisted phases due to their unconventional orbital-dominant magnetism and non-metallic conductivity. In Chapters 4 and 5 we will investigate magnetic heterostructures involving SmN, $\mathrm{NdN}$ and GdN, and provide evidence for the existence of these twisted phases. 
Chapter 2 Theoretical Background 


\section{Chapter 3}

\section{Experimental Techniques}

\subsection{Synchrotron Techniques}

The main experimental tool used in this thesis is x-ray magnetic circular dichroism (XMCD). It is one of the most powerful tools for studying magnetism, providing the ability to measure element-resolved magnetization as well as distinguish spin and orbital contributions to the magnetization. The technique has only been in use since the mid 1980s, with the introduction of elliptically polarized x-rays at undulator beamlines in synchrotron facilities [40]. The development of the XMCD sum rules in the early 1990s has made it possible to extract the spin and orbital magnetic moments directly from the experimental spectra.

Synchrotron radiation is a specific type of radiation which occurs when ultra-relativistic ${ }^{1}$ charged particles are accelerated in circular orbits by magnetic fields. In this energy regime, the emitted radiation from the particle has a much narrower angular and spatial divergence compared to a non-relativistic particle. The main advantage of using a synchrotron is its range of energies, spanning from about $10 \mathrm{eV}$ to $50,000 \mathrm{eV}$, and the high brilliance compared to lab based sources. Modern undulator beamlines now provide elliptically polarized light over a wide range of x-ray energies. In this work, we consider the soft and hard X-ray regimes, in the range of $200-1500 \mathrm{eV}$ and over $1500 \mathrm{eV}$, respectively. These two ranges contain the binding energies of the

\footnotetext{
${ }^{1}$ i.e. $\gamma=\frac{1}{\sqrt{1-v^{2} / c^{2}}} \gg 1$
} 


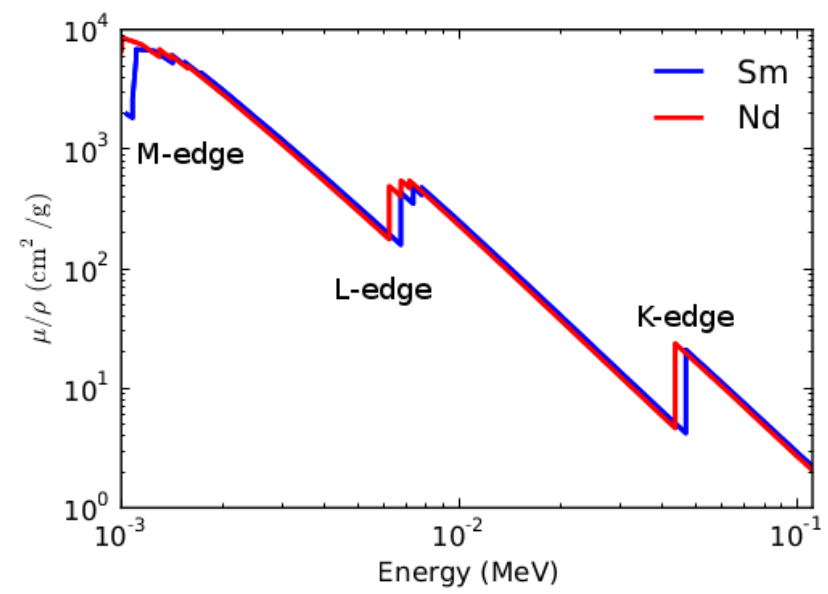

Figure 3.1: Mass attenuation coefficient $\mu / \rho=\sigma^{\text {tot }} / m_{A}$ for elemental Sm and $\mathrm{Nd}$, where $\rho$ is the density, $m_{A}$ is the mass, and $\sigma^{\text {tot }}$ is the total absorption cross section. Data from NIST.

rare-earth $3 d$ and $2 p$ shells we are interested in. XMCD is a type of x-ray absorption spectroscopy (XAS), and so we begin with a discussion of XAS in the rare-earths.

\section{X-ray absorption spectroscopy (XAS)}

XAS can be most simply described as an absorption process where we measure the transmitted X-ray intensity before $\left(I_{0}\right)$ and after $(I(E, d))$ passing through a sample of thickness $d$. The relationship is given by

$$
I(E, d)=I_{0} e^{-\mu(E) d},
$$

where $\mu(E)$ is the linear absorption coefficient, which depends on the energy $E$ of the X-ray photons. The absorption coefficient is related to the X-ray absorption cross-section $\sigma^{\text {abs }}$ through $\mu(E)=\rho_{A} \sigma^{\text {abs }}(E)$, where $\rho_{A}$ is the number of atoms per unit volume. If the X-ray energy is resonant with an atomic transition between two states, i.e. $E=E_{f}-E_{i}$, then $\sigma^{\text {abs }}(E)$ increases dramatically at this resonance, known as the absorption edge. The change in $\sigma^{\text {abs }}$ from right before this absorption threshold to immediately after is known as 


\begin{tabular}{lcccc}
\hline Edge & & $\mathrm{Nd}$ & $\mathrm{Sm}$ & $\mathrm{Gd}$ \\
\hline $\mathrm{L}_{2}$ & $2 p_{1 / 2} \rightarrow 5 d$ & $6,722 \mathrm{eV}$ & $7,312 \mathrm{eV}$ & $7,930 \mathrm{eV}$ \\
$\mathrm{L}_{3}$ & $2 p_{3 / 2} \rightarrow 5 d$ & $6,208 \mathrm{eV}$ & $6,459 \mathrm{eV}$ & $7,243 \mathrm{eV}$ \\
$\mathrm{M}_{4}$ & $3 d_{3 / 2} \rightarrow 4 f$ & $1,003 \mathrm{eV}$ & $1,111 \mathrm{eV}$ & $1,222 \mathrm{eV}$ \\
$\mathrm{M}_{5}$ & $3 d_{5 / 2} \rightarrow 4 f$ & $980 \mathrm{eV}$ & $1,083 \mathrm{eV}$ & $1,190 \mathrm{eV}$ \\
\hline
\end{tabular}

Table 3.1: Rare-earth absorption edges

the absorption edge-jump (Figure 3.1 shows edge-jumps for elemental Sm and $\mathrm{Nd}$ ). Each atom has a specific energy for the absorption edge corresponding to the electronic transition.

This demonstrates the usefulness of synchrotron radiation, which provides the ability of tuning the X-ray energy $E$ to specific absorption edges. This means different elements can be selected, as well as different absorption edges of the same element. The edges are labelled by the principal quantum number $n=0,1,2 \ldots=K, L, M \ldots$ with a subscript to distinguish spin-orbit split states according to $l \pm s$.

In this work we examine four of the rare-earth absorption edges. In the soft x-ray regime are the $\mathrm{M}_{4}$ and $\mathrm{M}_{5}$ edges, corresponding to transitions from $3 d_{3 / 2} \rightarrow 4 f$ and $3 d_{5 / 2} \rightarrow 4 f$, where the core level $3 d$ states are spin-orbit split according to $j=2 \pm 1 / 2$. In general we will refer to the two edges together as the M-edge. In the hard X-ray regime are the $\mathrm{L}_{2}$ and $\mathrm{L}_{3}$ edges (or L-edge for both), which correspond to transitions from $2 p_{1 / 2} \rightarrow 5 d$ and $2 p_{3 / 2} \rightarrow 5 d$, respectively. Table 3.1 lists the $\mathrm{x}$-ray energies at which the absorption edges occur.

To gain a more fundamental insight, we can look at a more detailed quantum mechanical picture (for a full account, see e.g. Stohr [41] or de Groot \& Kotani [42]). The cross-section for absorption is proportional to the probability of an electron in an initial state $\left|\psi_{i}\right\rangle$ going to a final state $\left|\psi_{f}\right\rangle$ due to an incident X-ray photon. This probability $P_{f i}$ is given in first order by Fermi's golden rule,

$$
P_{f i}=\frac{2 \pi}{\hbar}\left|\left\langle\psi_{f}|T| \psi_{i}\right\rangle\right|^{2} \delta\left(E_{f}-E_{i}-\hbar \omega\right) \rho\left(E_{f}\right)
$$

where the Dirac delta function preserves conservation of energy, $\hbar \omega$ is the 


\section{Chapter 3 Experimental Techniques}

photon energy, and $\rho\left(E_{f}\right)$ is the density of final states. If there are multiple final states, a sum over each final state must be included. Here $T$ represents the transition operator for the photon, which is given by

$$
T=\frac{e}{m_{e}} \mathbf{p} \cdot \boldsymbol{\epsilon} e^{i \mathbf{k} \cdot \mathbf{r}}
$$

where $\boldsymbol{\epsilon}$ is the polarization of the photon, $\mathbf{k}$ is its wavevector, and $\mathbf{p}$ the electron momentum operator. If $|\mathbf{k} \cdot \mathbf{r}| \ll 1$, then we approximate the exponential as $\exp (i \mathbf{k} \cdot \mathbf{r}) \approx 1$. This is known as the dipole approximation and is well justified at the rare-earth M-edge. For the rare-earths the atomic radii are approximately $\langle r\rangle_{3 d} \approx 0.15 \AA$, and the typical M-edge energy of $1 \mathrm{keV}$ corresponds to $k=E / \hbar c \approx 5 \times 10^{9} \mathrm{~m}^{-1}$, thus $\mathbf{k} \cdot \mathbf{r} \approx 0.0008$. At the L-edge, where $E \sim 7 \mathrm{keV}$ and $\langle r\rangle_{2 p} \approx 5 \times 10^{-12} \mathrm{~m}$, we find $\mathbf{k} \cdot \mathbf{r} \approx 0.002$.

The absorption cross section $\sigma^{\text {abs }}$ is proportional to $P_{f i}$, and is given by

$$
\sigma^{a b s}=4 \pi^{2} \alpha \hbar \omega\left|\left\langle\psi_{f}|\mathbf{r} \cdot \boldsymbol{\epsilon}| \psi_{i}\right\rangle\right|^{2} \delta\left(E_{f}-E_{i}-\hbar \omega\right) \rho\left(E_{f}\right),
$$

where $\alpha \approx 1 / 137$ is the fine structure constant. The polarization in the matrix element $\left\langle\psi_{f}|\mathbf{r} \cdot \boldsymbol{\epsilon}| \psi_{i}\right\rangle$ plays an important role. For example, the photon polarization tensor has three components $\boldsymbol{\epsilon}_{z}^{q}$ corresponding to linear polarization $(q=0)$, and both right $(q=+1)$ and left $(q=-1)$ circular polarizations. In terms of position basis vectors given by $\hat{\mathbf{e}}_{i}(i=x, y, z)$, we have $\boldsymbol{\epsilon}_{z}^{0}=\hat{\mathbf{e}}_{z}$, and $\boldsymbol{\epsilon}_{z}^{ \pm 1}=\left(\hat{\mathbf{e}}_{x} \pm i \hat{\mathbf{e}}_{y}\right) / \sqrt{2}$. The dipole operator can then be written

$$
\begin{aligned}
\mathbf{r} \cdot \boldsymbol{\epsilon}_{z}^{0} & =z=r \cos \theta=r \sqrt{\frac{4 \pi}{3}} Y_{1,0}(\theta, \phi) \\
\mathbf{r} \cdot \boldsymbol{\epsilon}_{z}^{ \pm 1} & =\mp \frac{1}{\sqrt{2}}(x+i y)=\mp r \frac{1}{\sqrt{2}} \sin \theta e^{ \pm i \phi}=r \sqrt{\frac{4 \pi}{3}} Y_{1, \pm 1}(\theta, \phi)
\end{aligned}
$$

Where the $Y_{l, m}(\theta, \phi)$ are spherical harmonics and the angles $\theta, \phi$ correspond to the usual definition of spherical coordinates. ${ }^{2}$ The presence of these spherical

\footnotetext{
${ }^{2}$ For circular polarization, if the photon wavevector $\mathbf{k}$ is in the $z$ direction, then $\boldsymbol{E}$ is in the $x-y$ plane. For linear polarization, $\boldsymbol{E}$ must again be perpendicular to $\mathbf{k}=k \hat{\mathbf{e}}_{z}$, e.g. the $x$ or $y$ direction. But to retain the convention that $J_{z}$ is diagonal in the $\left\{\left|J M_{J}\right\rangle\right\}$ basis, we can rotate the coordinate system so that $\mathbf{k}=k \hat{\mathbf{e}}_{x}$ and $\boldsymbol{E}$ (and thus $\boldsymbol{\epsilon}^{0}$ ) is in the $z$ direction, giving the correct selection rule for linear polarization.
} 
harmonics is responsible for polarization-dependent selection rules, which only allow certain transitions to take place. Because the states involved in the transition are products of radial and angular wave functions, according to $\left|\psi_{i}\right\rangle=\left|R_{n}^{i}\right\rangle\left|J M_{J}\right\rangle$, we see that $Y_{l, m}(\theta, \phi)$ acts only on the angular wave function and $r$ on the radial wave function. Thus the absorption cross-section depends on the polarization by

$$
\sigma^{\text {abs }} \propto\left|\left\langle J^{\prime} M_{J^{\prime}}\left|\epsilon_{z}^{q}\right| J M_{J}\right\rangle\right|^{2}
$$

The result is that $\left\langle J^{\prime} M_{J^{\prime}}\left|\boldsymbol{\epsilon}_{z}^{q}\right| J M_{J}\right\rangle$ is only non-zero if the following relations are satisfied:

$$
\begin{aligned}
\Delta M & =M_{J^{\prime}}-M_{J}=q \\
\Delta J & =J^{\prime}-J= \pm 1,0 \\
\Delta S & =0, \quad \Delta L= \pm 1,
\end{aligned}
$$

however $\Delta J=0$ transitions are allowed only if $J \neq 0$. The selection rule $\Delta S=0$ means no spin flips are allowed in the transition. In the normal XAS measurement, the polarization is linear $(q=0)$, so there are no transitions between different $M_{J}$ states. On the other hand, for circularly polarized light $\Delta M= \pm 1$ for right (+) and left (-) polarizations. As we shall see later, these transitions between values of $M_{J}$ are the basis of XMCD.

\subsubsection{M-edge absorption}

At the rare-earth M-edge, an electron in the $3 d$ shell is excited to one of the empty $4 f$ levels. This creates a hole in the core $3 d$ states which can interact with the $4 f$ electrons due to $3 d-4 f$ overlap. Because of this, the single electron picture of the absorption process is no longer valid, and the multi-electron initial and final states (labeled by the total $S, L, J$ quantum numbers) must be considered. We denote this configuration dependent process by $4 f^{n} \rightarrow 3 d^{9} 4 f^{n+1}$, where $n$ is the number of $4 f$ electrons in the ground state. The $3 d$ hole then couples to the $f^{n+1}$ electrons so that the total angular momentum of the final state results in a variety of multiplets with different 


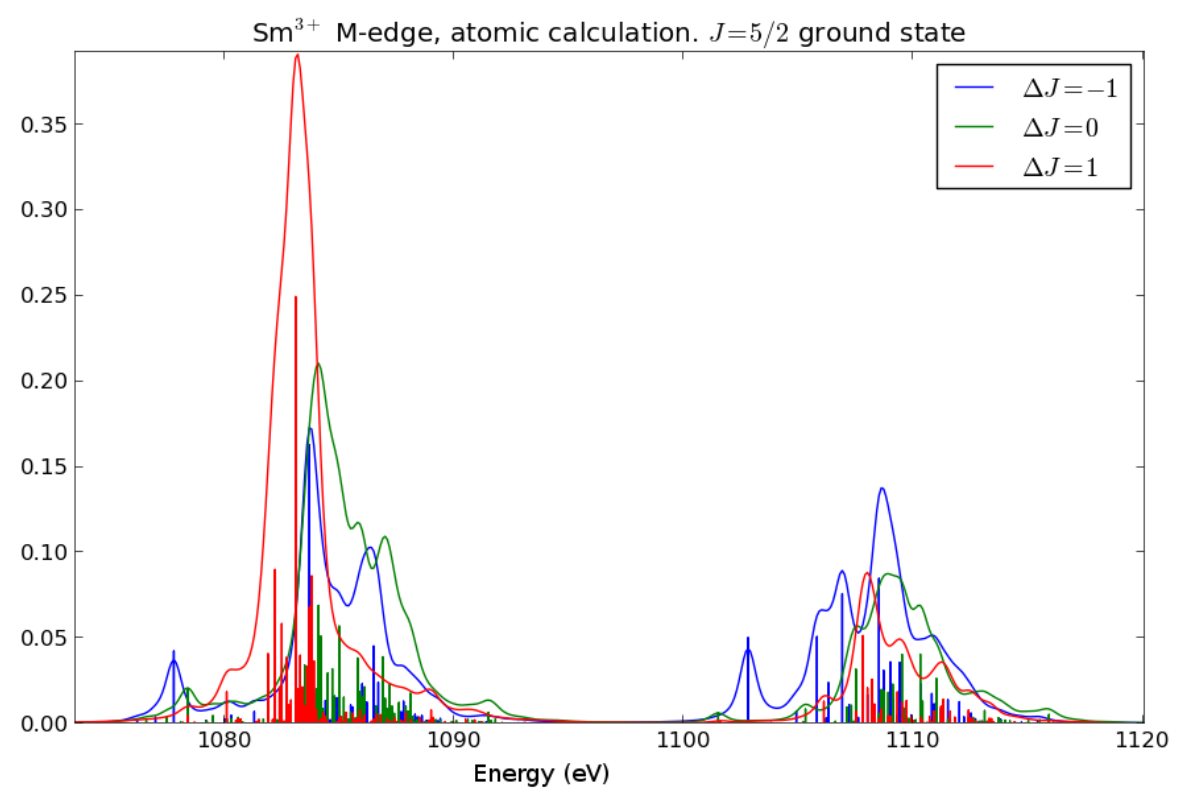

Figure 3.2: Calculated $\mathrm{Sm}^{3+}$ XAS spectra. Vertical bars represent dipole allowed transitions and their strength, the curves simulate broadening effects due to instrumental and lifetime effects, calculated using the program Missing [44].

term symbols ${ }^{2 S+1} L_{J}$. There can be a large number of multiplets, for example $\mathrm{Sm}^{3+}$ has 2,725 different multiplets in the final state $3 d^{9} f^{6}$ configuration. The dipole selection rule $\Delta J=0, \pm 1$ reduces the actual number of final states which are accessible to 946 for $\mathrm{Sm}^{3+}$ [43]. For this reason, the resulting absorption spectrum reflects the many possible final states; they can be included in $\sigma^{\text {abs }}$ by a sum over the final states;

$$
\sigma^{\mathrm{abs}}=4 \pi^{2} \hbar \omega \sum_{f}\left|\left\langle\psi_{f}|\mathbf{r} \cdot \boldsymbol{\epsilon}| \psi_{i}\right\rangle\right|^{2} \delta\left(E_{f}-E_{i}-\hbar \omega\right) \rho\left(E_{f}\right)
$$

Figure 3.2 shows a simulated absorption spectrum at the M-edge of $\mathrm{Sm}^{3+}$ for the three different dipole allowed transitions. The spectrum is composed of hundreds of closely spaced transitions, due to the hundreds of matrix elements $\left\langle\psi_{f}|\mathbf{r} \cdot \boldsymbol{\epsilon}| \psi_{i}\right\rangle$. The density of states factor $\rho\left(E_{f}\right)$ in the cross section only serves to broaden each line, however. The transitions are concentrated around the 
two edges, the lower energy $\mathrm{M}_{5}$ corresponding to the hole with $J=5 / 2$ and the $\mathrm{M}_{4}$ corresponding to the $3 d$ hole with $J=3 / 2$. The rare-earth M-edge can be simulated using Cowans code [21], which is based on atomic multiplet calculations [45].

\subsubsection{L-edge absorption}

At the L-edge the $2 p^{6} \rightarrow 2 p^{5} 5 d^{1}$ transition is not dominated by multiplet effects, so the single electron picture of a core $2 p$ electron transitioning into the band-like $5 d$ states describes the absorption process. This is because the overlap between the $2 p$ core hole and $5 d$ states is small compared to the strong core-hole- $4 f$ interaction at the M-edge. The result is that the absorption crosssection measures the unoccupied partial density of states (PDOS) $\rho\left(E_{f}\right)$ of the $5 d$ level ${ }^{3}$

$$
\sigma^{\mathrm{abs}} \propto \mathcal{M} \rho\left(E_{f}\right)
$$

where $\mathcal{M}=\left|\left\langle\psi_{f}|\mathbf{r} \cdot \boldsymbol{\epsilon}| \psi_{i}\right\rangle\right|^{2}$ is approximately constant over the energy range of the absorption edge. Because of the band-like nature of the $5 d$ states, band structure calculations are needed to simulate the PDOS. In the RENs the strong electron correlations from the $4 f$ states have to be accounted for when calculating the PDOS, which makes band structure calculations difficult $[14$, $46]$.

\subsubsection{Detection methods}

At the $\mathrm{M}$ and $\mathrm{L}$ edges, indirect methods of measuring the absorption are used instead the transmission mode, where $I=I_{0} e^{-\mu(E)}$. Once the absorption process takes place, the core hole can decay via radiative and non-radiative decay channels. The non-radiative channel is via Auger and Coster-Konig processes. The number of such secondary electrons produced is then proportional to the $\mathrm{X}$-ray absorption cross section $\sigma^{\text {abs }}$, this is known as the total electron yield (TEY). The electrons are usually measured by the drain current or by using a

\footnotetext{
${ }^{3}$ The selection rules prevent transitions into the full unoccupied density of states.
} 


\section{Chapter 3 Experimental Techniques}

channeltron electron multiplier. The resulting intensity is then normalized to the incident beam. For an electron mean-free path $\lambda_{e}$ and the X-ray photon absorption length $\lambda_{x}$ (i.e. the inverse of the absorption coefficient, $\mu=\lambda_{x}^{-1}$ ) the absorption intensity is

$$
I_{\mathrm{TEY}}=I_{0} G \frac{\lambda_{e}}{\lambda_{e}+\lambda_{x} \cos \theta}
$$

where $\theta$ is the measured angle relative to the surface normal, $I_{0}$ is the incident beam intensity, and $G$ is the electron gain factor. As long as $\lambda_{x} \cos \theta \gg \lambda_{e}$ the measured signal is proportional to the absorption coefficient, $I_{\mathrm{TEY}} \propto \mu(E)$. Typically $\lambda_{e} \approx 1-3 \mathrm{~nm}$, while $\lambda_{x}$ is generally of order $100 \mathrm{~nm}$, though $\lambda_{x}$ may decrease significantly at the absorption edge in rare-earths [45]. However even at the absorption edge $\lambda_{x}>\lambda_{e}$. In grazing incidence measurements $\lambda_{x}$ may become comparable to $\lambda_{e}$ and the spectrum can become distorted. [41, 47]. These effects must be corrected for when applying the sum rules, however for temperature or magnetic field dependent measurements these effects are less important.

When radiative decay occurs, the emitted X-rays can be measured via a channeltron electron multiplier. The photon flux is called total fluorescence yield (TFY). In the limit that the $\lambda_{x}$ is larger than both the emitted fluorescence absorption length, $\lambda_{x^{\prime}}$, and background fluorescence absorption length, $\lambda_{B}$,

$$
I_{T F Y}=c_{1}+c_{2} \mu,
$$

where $c_{1}$ represents a constant background due to e.g. a capping layer or substrate [48]. Saturation effects are more common in the TFY mode due to the similar length scales of $\lambda_{x}$ and $\lambda_{x^{\prime}}$, and generally the sum rules are not applicable.

A variant of the TFY mode is the partial fluorescence yield (PFY), where specific fluorescence decay channels are measured with an energy selective spectrometer. For instance, at the rare-earth $\mathrm{L}_{3}$ edge the most prominent radiative decay channel occurs when the $2 p_{3 / 2}$ hole is filled with an electron transition 


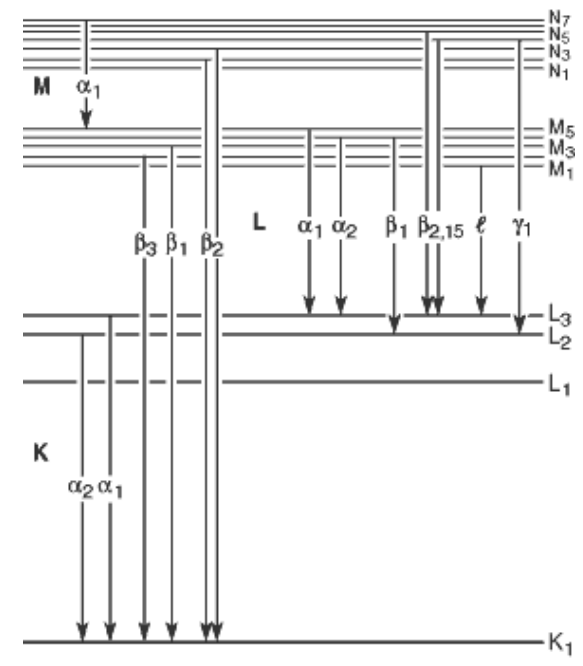

Figure 3.3: Electronic transitions corresponding to various emission lines, taken from [40]. The spectroscopic notation is used, where $K, L, M \ldots=$ $0,1,2 \ldots$ refers to $s, p, d$-states.

from a $3 d_{5 / 2}$ state with the $\mathrm{L} \alpha_{1}$ emission line at 5,230 eV. Similarly, at the $\mathrm{L}_{2}$ edge the $\mathrm{L}_{\beta_{1}}$ emission line at $5,722 \mathrm{eV}$ is the most prominent radiative decay channel (see Figure 3.3 for emission lines). This method has the advantage of removing unwanted background signals from different atomic species which have absorption edges close by, for instance in multilayers. Even if there are no nearby absorption edges, higher order harmonics in the X-ray beam can excite edges far away in energy resulting in unwanted spectral features, making PFY a very useful tool.

\subsubsection{XMCD}

The description of XAS in the preceding sections will simplify our discussion of XMCD, which is essentially an application of XAS. The XMCD signal $\Delta I$ can be defined as

$$
\Delta I=I_{+}(\mathbf{H})-I_{-}(\mathbf{H})
$$

where $I_{ \pm}$is the XAS spectrum taken with positive $(q=+1)$ or negative $(q=-1)$ helicity photons, and the magnetic field $\mathbf{H}=(0,0,-H)$ is in the 
$-\hat{\mathbf{z}}$ direction. The photon momentum direction and spin quantization axis are taken in the $+\hat{\mathbf{z}}$ direction. ${ }^{4}$ This can equivalently be written as

$$
\Delta I=I_{-}(-\mathbf{H})-I_{+}(-\mathbf{H})
$$

when the field is reversed. This definition corresponds to the difference between antiparallel and parallel arrangements of the spin and magnetic field. ${ }^{5}$ We will generally keep $\mathbf{H}$ fixed, so use the simpler notation $I_{ \pm}$to refer to the absorption spectra taken with different polarizations. $I_{0}$ refers to the absorption spectra with linear polarization parallel to $\hat{\mathbf{z}}$.

\subsubsection{The two-step XMCD model}

The basic picture of XMCD is often described in a two step model [41], usually for transition metal systems. This standard view is not strictly applicable to the rare-earths, but nevertheless provides a useful intuitive picture of the $\mathrm{XMCD}$ process, and so we review XMCD in transition metal systems before describing the XMCD process in the rare-earths.

We consider XMCD at the $\mathrm{L}_{2}$ and $\mathrm{L}_{3}$ edge $2 p \rightarrow 3 d$ transitions which occur in the $3 d$ transition metals. In the first step, a polarized photon excites an electron from the $2 p$ state. The spin-orbit splitting of the $2 p$ state means at the $\mathrm{L}_{2}$ edge, where $j=l-s=1 / 2$, the spin and orbital momentum are antiparallel, and at the $\mathrm{L}_{3}$ edge, where $j=3 / 2$, the spin and orbital moments are parallel. Photons of positive helicity $q=+1$ will have a higher transition probability of exciting spin-up electrons, and negative helicity photons $(q=-1)$ will preferentially excite spin-down electrons (see Figure 3.4 ). This is because the matrix elements are largest for parallel helicity, spin, and orbital momentum. ${ }^{6}$

The second step of the process occurs when the spin-polarized photoelectrons transition into the unoccupied $3 d$ states. If the bands are spin-polarized due to exchange splitting, the majority (spin-down in this case) and minority

\footnotetext{
${ }^{4}$ The helicity is defined as the projection of the photon spin onto its momentum vector $(\hat{\mathbf{p}} \cdot \mathbf{S})$

${ }^{5}$ The spin-moment is antiparallel to the expectation value of the spin : $\mathbf{m}_{S}=-2 \mu_{B} \mathbf{S}$.

${ }^{6}$ Because the dipole operator $\mathbf{r} \cdot \boldsymbol{\epsilon}$ does not act on spin, but only on orbital angular momentum, the spin-orbit coupling of the core level is responsible for the spin polarization.
} 


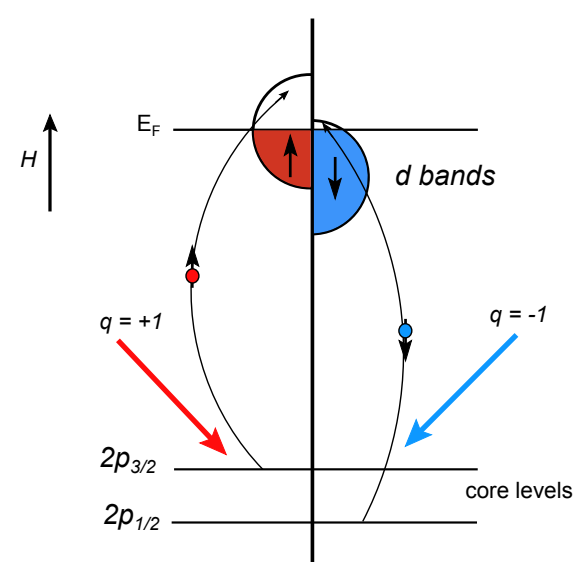

Figure 3.4: A sketch of the XMCD process, showing only the dominant transitions into the unoccupied exchange-split $d$ states. The asymmetry in absorption between x-rays with $q= \pm 1$ polarization results in the dichroic signal.

(spin-up) bands form. The excited $2 p$ photoelectrons cannot change their spin due to the selection rule $\Delta S=0$, so the unoccupied $d$ states act as a spin detector. The imbalance in occupation is proportional to the magnetization $\left(|\mathbf{m}| \propto \rho^{\uparrow}(E)-\rho^{\downarrow}(E)\right)$, and thus the imbalance in $I_{ \pm}$is proportional to the magnetic moment per atom projected on the quantization axis; $\Delta I \propto|\mathbf{m}| \cos \varphi$, where $\varphi$ is the angle between the magnetic field and quantization axis. Because the exchange splitting $\varepsilon$ is small compared to the overall bandwidth, we can write $\rho^{\uparrow}(E)=\rho^{\downarrow}(E+\varepsilon)$, and expanding to first order find $\Delta I \propto \frac{d \rho^{\downarrow}(E)}{d E}$. The $\mathrm{XMCD}$ signal is thus proportional to the derivative of the density of states of the final $d$ levels. This is observed experimentally in Chapters 5 and 6 at the rare-earth L-edge where the XMCD has a dispersive lineshape.

To summarize, circular dichroism at a spin-orbit split edge originates from two effects: the spin-orbit splitting of the core levels and the exchange-splitting of the unoccupied final states. The process is of course element specific, which gives XMCD a major advantage over standard magnetometry methods which cannot distinguish individual elemental contributions to the magnetization. 


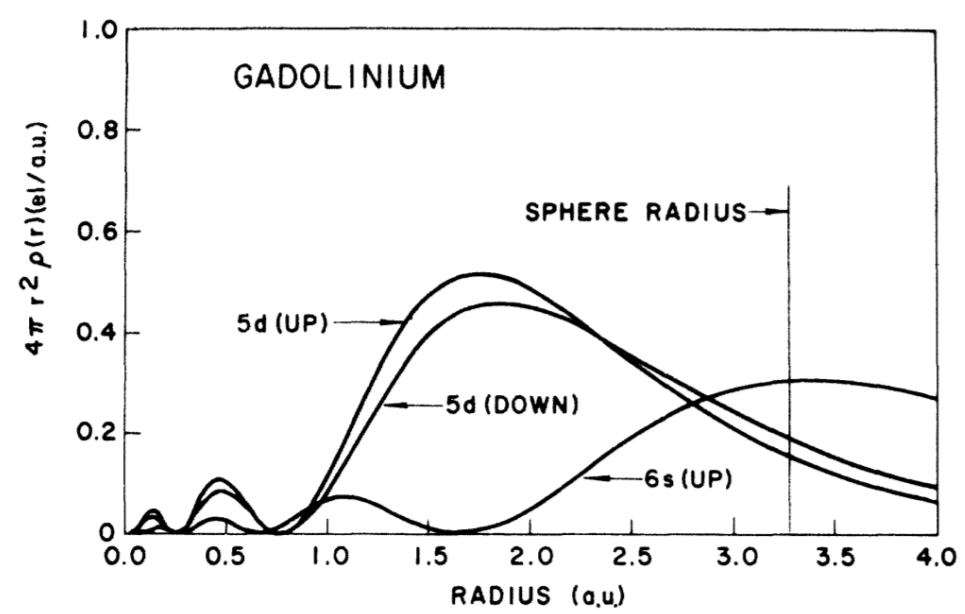

Figure 3.5: Calculated atomic Gd $5 d$ radial wave functions with spin up and spin down, showing the spin-up wave function pulled slightly closer to the nucleus, from Ref. [49].

\subsubsection{Rare-earth L-edge XMCD}

The rare-earth L-edge probes $2 p \rightarrow 5 d$ transitions. As we saw in Chapter 2 , the unoccupied $5 d$ states mediate exchange between ions in a superexchange process. The $5 d$ XMCD signal is thus a measure of this indirect exchange mechanism. A further difference from the transition metal case is that the matrix elements of the transition are spin-dependent.

In the RENs, the $5 d$ bands are exchange-split into majority and minority bands. These bands overlap with the $4 f$ states enough that there is an exchange interaction between the $5 d$ and $4 f$ majority spin bands. Because the exchange energy is negative, the $5 d$ majority band then contracts towards the nucleus, which increases the overlap with the $4 f$ band and lowers the energy. This can be seen in Figure 3.5, where a calculation for atomic Gd shows the contraction of the Gd $5 d$ spin-up radial wavefunction.

The contracted $5 d$ majority band then overlaps more strongly with the $2 p$ orbitals, leading to an enhancement in transitions through the majority spin channel, resulting in the spin dependence of the $2 p \rightarrow 5 d$ transition matrix elements. This spin-dependence of the matrix elements leads to a large branching ratio between the $\mathrm{L}_{2}$ and $\mathrm{L}_{3}$ edges, with $\mathrm{L}_{2} / \mathrm{L}_{3} \sim 10$ for the light rare-earths, 
while the ratio is reversed for the heavy rare-earths. This makes direct application of the sum rules impossible at the rare-earth L-edge, giving the wrong sign and magnitude of the $5 d$ spin and orbital moments [50-53].

Additionally, an electric quadrupole transition $(2 p \rightarrow 4 f)$ is also visible in the XMCD spectrum of the light rare-earths due to relatively weak dipole transition contributions [53], allowing a direct probing of the $4 f$ magnetism. The quadrupole feature is usually much weaker than the dipole transitions but is often still visible. In Chapter 5 we shall see that the $2 p \rightarrow 4 f$ quadrupole transition at the $\mathrm{Sm} \mathrm{L}_{3}$ edge is visible.

\subsubsection{Rare-earth M-edge XMCD}

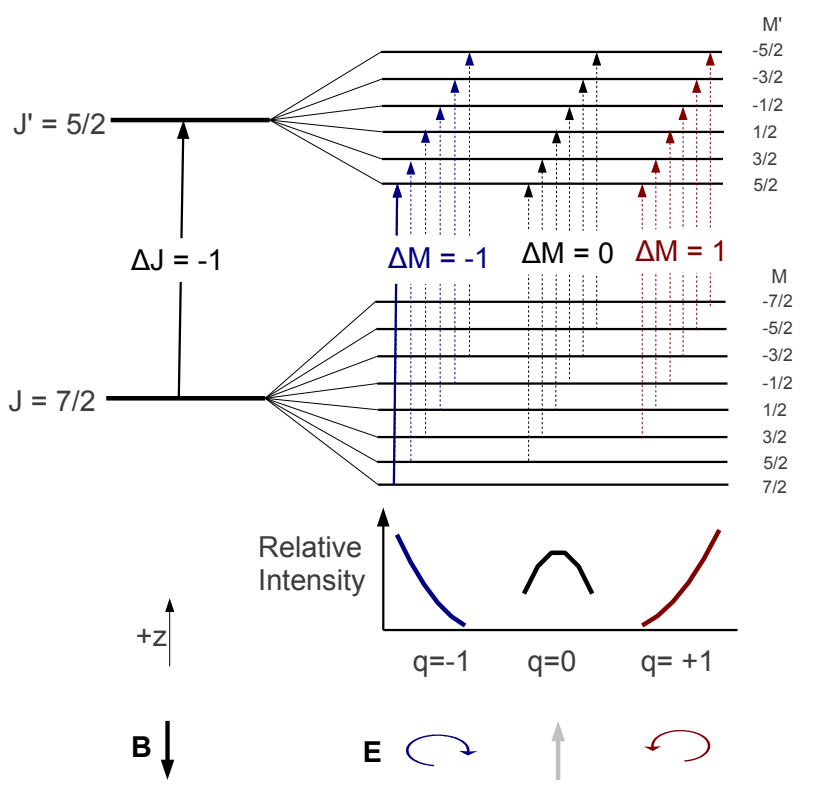

Figure 3.6: The initial $J=7 / 2\left(f^{13}\right.$ configuration $)$ and final state $J=5 / 2$ ( $d^{9} f^{14}$ configuration) multiplet of $\mathrm{Yb}^{3+}$. When $T=0$, only the $J=M=7 / 2$ ground state is occupied, and there is only one transition (bold) to the $J^{\prime}=$ $M^{\prime}=5 / 2$ final state. After Goedkoop et al. [54]

In M-edge XMCD of the rare-earths, the single-electron band picture we described previously is complicated by the multiplet structure. In this case, it is 
easier to view the two-step XMCD process from an atomic point of view instead of the energy band point of view, though the two pictures are equivalent. We give an example of the rare-earth XMCD process in the simplest case of $\mathrm{Yb}^{3+}$, with a ground state $f^{13}$ multiplet given by $S=1 / 2, L=3, J=7 / 2$ [54]. The same principles apply to the other rare-earths, which have much larger number of dipole-allowed transitions.

We start with the Hunds' rule ground state multiplet, in the presence of a magnetic field. The presence of $\mathbf{H}$ lifts the $2 J+1$ degeneracy of the $J$ multiplet, and the sublevels are split according to $M=-J,-J+1, \ldots, J$ (see Figure 3.6). In the ground state, only the lowest energy $M$ level is occupied, given by $M=7 / 2$ when $\mathbf{H}$ is in the $-\hat{z}$ direction. From this $J=M=7 / 2$ level the dipole selection rule states that $\Delta J=0, \pm 1$, and depending on the photon helicity, $\Delta M= \pm 1$.

If we examine the possible final states, we see there is only one, $3 d^{9} f^{14}$, which is equivalent to a single $3 d$ hole with quantum numbers $S=1 / 2, L=$ $2, J=5 / 2$. This means that there is only one allowed transition with $\Delta J=-1$ and $\Delta M=-1$ to the final state $J=M=5 / 2$ level. Thus photons with $q=+1$ excite no photoelectrons and there is no absorption for $I_{+}$. The XMCD spectrum is just $\Delta I=-I_{-}$and consists of one transition (Figure 3.6). The same reasoning determines the transitions from $\mathrm{Sm}^{3+}$, with a ground state labeled by $J=M=5 / 2$ in a magnetic field. However there are 946 final states, with varying strengths, making the XMCD spectrum far more complex than for $\mathrm{Yb}^{3+}$. For example, atomic multiplet calculations of the XMCD spectra for $\mathrm{Gd}^{3+}$ and $\mathrm{Sm}^{3+}$ are shown in Figure 3.7, showing the three separate $\Delta J$ contributions to the total XMCD signal at the M-edge.

\subsubsection{XMCD sum rules}

The resulting XMCD spectrum $\Delta I$ can be related to the spin $\left(m_{S}=-2 \mu_{B}\left\langle S_{z}\right\rangle\right)$ and orbital $\left(m_{L}=-\mu_{B}\left\langle L_{z}\right\rangle\right)$ moments of the occupied states via the sum rules developed by Thole et al. [55] and Carra et al. [56]. We write them specifically for the rare-earth M-edge for simplicity, though they can be written similarly for any edge. In the case of the M-edge, the moments obtained from the sum 

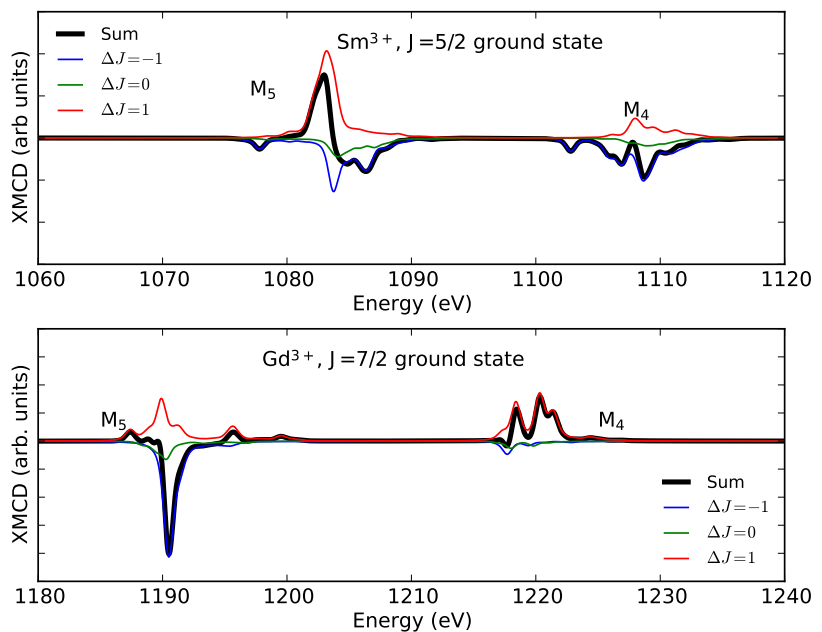

Figure 3.7: Atomic multiplet calculations of $\mathrm{Gd}^{3+}$ and $\mathrm{Sm}^{3+}$ XMCD spectra including simulated lifetime broadening and instrumental resolution broadening. The dipole-allowed transitions to the final states with $\Delta J=0, \pm 1$ are shown, with the sum over all these final states representing the XMCD spectrum. Calculated using Missing [44].

rules refer to the occupied $4 f$ states only. The isotropic absorption can be written as $I_{S}=I_{+}+I_{-}+I_{0}$, and

$$
A_{4,5}=\int_{M_{4}, M_{5}} d E I_{S}, \quad \Delta A_{4,5}=\int_{M_{4}, M_{5}} d E \Delta I
$$

are the integrals of $I_{S}$ and $\Delta I$ over either the $M_{4}$ or $M_{5}$ edge energies. The orbital sum rule is given by

$$
\left\langle L_{z}\right\rangle=n_{h} \frac{\Delta A_{4}+\Delta A_{4}}{A_{4}+A_{5}}
$$

where $n_{h}=14-n$ is the number of $4 f$ shell holes in the initial state (denoted $\left.4 f^{n}\right)$. The spin sum rule is slightly more complex and is given by

$$
\left\langle S_{z}\right\rangle=n_{h} \frac{\Delta A_{5}-\frac{3}{2} \Delta A_{4}}{A_{4}+A_{5}}+6\left\langle T_{z}\right\rangle
$$

where $T_{z}$ is the magnetic dipole operator which is related to the asphericity of the charge distribution. For the rare-earths this term is significant and must 


\section{Chapter 3 Experimental Techniques}

be calculated in order to apply the sum rules. In $3 d$ transition metal systems the magnetic dipole contribution is very small and often ignored. There are many limitations to applying the sum rules, as outlined in de Groot \& Kotani [42], however they provide the theoretical link between the XMCD spectra and the orbital and spin moments. In the RENs information on the overall sign of $\left\langle L_{z}+2 S_{z}\right\rangle$ can be determined from XMCD. This is especially important in the case of $\mathrm{SmN}$, where the the spin and orbital moments are nearly equal in magnitude but have opposite signs. By looking at the overall sign of the $\Delta I$ spectrum can tell if $m_{L}$ or $m_{S}$ is larger.

\subsection{Thin Film Growth}

The growth of rare-earth nitride samples is a challenge due to the propensity for the rare-earth elements to oxidize when in atmosphere, which is especially strong in the light rare-earths. In order to prevent oxidation of samples special preparation conditions must be in place. The methods of growing high quality rare-earth nitride thin films have recently been established in the laboratory at Victoria University, and are reviewed in detail by Natali et al. [2]. The most successful methods used include molecular beam epitaxy, pulsed-laser deposition, and dc/rf magnetron sputtering. The benefits of these methods are the use of high and ultra-high vacuum systems as inert gas environments which prevent contaminants in the growth process. In this thesis, we use a molecular beam epitaxy technique (MBE).

In the MBE growth of REN thin films, an ultra-high vacuum chamber with a base pressure of $\leq 10^{-8}$ Torr is used to minimize contaminants and prevent oxidation of the highly reactive rare earths. The basic operating principle can be summarized in the following way. High purity rare-earth metals are heated to the point of evaporation with either an electron gun or by using a thermal evaporator (a boron nitride crucible heated with tungsten filaments). At the same time ultra-high purity $\mathrm{N}_{2}$ gas is released into the chamber at a pressure between $10^{-5}$ and $10^{-4}$ Torr (another variant uses an $\mathrm{NH}_{3}$ nitrogen precursor [13]). A crystalline substrate is then mounted on a rotating platform and the REN forms on the surface of the substrate. The evaporation rate of the RE 
metal is measured using a quartz crystal balance. The temperature of the substrate, $\mathrm{N}_{2}$ pressure, and evaporation rate all must be carefully controlled to achieve high quality films. Epitaxial REN films are achieved at higher growth temperatures, while room temperature growth generally leads to textured polycrystalline films [57]. The number of nitrogen vacancies also depends on the growth parameters, and thus strongly affect the electric transport properties, while the magnetic properties are affected to a lesser degree. For GdN, the Curie temperature is seen to vary from $50 \mathrm{~K}$ with low carrier concentration $\left(<10^{18} \mathrm{~cm}^{-3}\right)$ to $70 \mathrm{~K}$ for concentrations greater than $3 \times 10^{20} \mathrm{~cm}^{-3}$. The mechanism is thought to be due to the formation of magnetic polarons, and not an RKKY exchange mechanism $[12,13]$.

For ex-situ study, however, a passivating capping layer must be used to prevent oxidation of the film. Typical choices are insulating GaN or AlN films, grown with the same technique as the REN films. Low energy ions from a Kaufman type ion source are used for the growth of GaN and AlN films, as well as EuN.

The choice of substrates plays an important role in the REN film growth. The rocksalt structure of the RENs suggests the use of substrates with cubic structure, however $\mathrm{MgO}$ and ytrria stabilized zirconia (YSZ) have shown the propensity for oxygen contamination due to oxygen diffusing into the REN film during high temperature growths. Non-cubic crystal structure substrates may also be used. High quality epitaxial REN films have been demonstrated on commercially available single crystal $c$-plane GaN and AlN buffer layers (100 nm thick) on sapphire substrates, while sapphire substrates may also be used to produce highly textured polycrystalline samples. The six-fold hexagonal symmetry results in a [111] oriented growth direction for the rocksalt structure RENs.

In this thesis, the specific growth conditions of each sample are listed in the experimental details section of the corresponding Chapter. 
Chapter 3 Experimental Techniques 


\section{Chapter 4}

\section{SmN and NdN Magnetism}

\subsection{Introduction}

In this chapter we investigate the ferromagnetic state of $\mathrm{SmN}$ and $\mathrm{NdN}$ using atomic calculations and compare with experimental data. These calculations consider only the $4 f$ states, ignoring other possible contributions such as conduction electron polarization to the magnetic moment. In the calculations we explore the results when excited $J$ multiplets and crystal field interactions are incorporated. We employ methods developed by Buschow, Dewijn et al. [35, 58-60] for Sm systems, which allows for calculation of crystal fields within arbitrary multiplets. We also use a self-consistent method to deal with the exchange interaction $[29,61]$ and carry out the first detailed study on $\mathrm{SmN}$ and NdN using this method.

The motivation for this endeavor is twofold. First, we would like to compare these crystal field calculations to band-structure calculations previously carried out on the RENs. Only a few such band-structure calculations exist for the RENs, and the calculated magnetic properties contradict experimental results in some cases. Second, a better understanding of how the spin and orbital moments contribute to the net moment in $\mathrm{SmN}$ and $\mathrm{NdN}$ will be useful for the XMCD study of SmN/GdN and NdN/SmN superlattices in Chapters 5 and 6 .

There are relatively few theoretical studies of $\mathrm{NdN}$ and $\mathrm{SmN}$, compared to GdN. This is perhaps because GdN is in some ways simpler, having the $S=J=7 / 2, L=0$ Hund's rule ground state. This $L=0$ state means that 
there is no spin-orbit coupling, thus the orbital wave function is spherical, leading to nearly zero interaction with the crystal field. ${ }^{1}$ In comparison the other RENs have $L \neq 0$ orbital wave functions, and in addition strong spinorbit coupling means that any effects from the crystal field are felt by both orbital and spin moments.

For SmN and NdN then, the coupling of the orbital moment to the lattice (crystal field interaction) and the spin-orbit coupling may have significant effects on the resulting magnetic properties in both ferromagnetic and paramagnetic phases. Our aim then, is to determine the degree to which the crystal field arising from the $\mathrm{N}$ ions influences the $4 f$ magnetic moments.

The calculations in the following were all carried out with original code written in Python by the author.

\subsection{SmN Magnetism}

\subsubsection{Experimental background}

Before proceeding to the calculations, we review some experimental $\mathrm{SmN}$ data. The most careful examination of SmN magnetization in thin films was carried out by Meyer et al. [30], who demonstrated that $\mathrm{SmN}$ has a ferromagnetic ground state with an ordered moment of $m=0.035 \mu_{B}$ per $\mathrm{Sm}^{3+}$ below the Curie temperature of $27 \pm 3 \mathrm{~K}$. Some of the low temperature hysteresis curves are shown in Figure 4.1. We note that this moment is considerably smaller than the free-ion moment of $\mu_{B} g_{J} J=0.74 \mu_{B}$. The paramagnetic susceptibility follows a Curie-Weiss law with a temperature independent van Vleck contribution, with an effective paramagnetic moment of $0.45 \mu_{B}$ per ion, smaller than the paramagnetic $\mu_{B} g_{J} \sqrt{J(J+1)}=0.85 \mu_{B}$ free-ion value. These findings agreed well with an earlier study of $\mathrm{SmN}$ that found an ordered moment of less than $0.1 \mu_{B}$ per ion and observed semiconducting behavior over the measured temperature range [5].

The paramagnetic moment in Ref. [30] was interpreted within a crystal

\footnotetext{
${ }^{1}$ In this case the small magnetic anisotropy is mainly due to dipolar interactions between ions.
} 
field model assuming that the cubic crystal field in SmN splits the ground state $J=5 / 2$ level into a doubly degenerate $\Gamma_{7}$ state and a four-fold degenerate $\Gamma_{8}$ state. Assuming only the $\Gamma_{7}$ doublet was occupied at temperatures just above the ordering temperature, the effective paramagnetic moment was calculated as $0.41 \mu_{B}$, using first order perturbation theory to calculate the susceptibility. The calculation was equivalent to restricting the sum over the Zeeman substates used in the derivation of the free-ion paramagnetic moment (Chapter 2 ). The good agreement with this calculated paramagnetic moment and the observed value suggests that the crystal field is significant and should be taken into account when calculating the ground state magnetic moment.

In an effort to separate the spin and orbital contributions to the magnetization, an XMCD study was carried out on a homogeneous $\mathrm{SmN}$ sample [19]. This study found that the orbital moment in $\mathrm{SmN}$ is parallel to the net magnetization, making $\mathrm{SmN}$ an orbital-dominant ferromagnet. Other $\mathrm{Sm}^{3+}$ systems do not necessarily share this orbital-dominant feature, which depends on the bonding environment of the ion and the contribution of conduction electrons. $\mathrm{SmAl}_{2}$ is known to be an orbital dominant ferromagnet, with a moment of $0.26 \mu_{B}$, while SmZn and SmCd have their net magnetization antiparallel to the orbital moment [29]. However, we note that in distinction to $\mathrm{SmN}$, all these systems are metallic, and the effects of conduction electron polarization and screening are quite different in $\mathrm{SmN}$.

\subsubsection{SmN calculations}

Many of the properties of $\mathrm{SmN}$ are dictated by the $\mathrm{Sm}^{3+}$ ion, which has been studied in a number of compounds in the past, including metallic Laves-phase compounds such as $\mathrm{SmAl}_{2}, \mathrm{Sm}_{1-x} \mathrm{Gd}_{x} \mathrm{Al}_{2}$, and $\mathrm{Sm}_{1-x} \mathrm{Nd}_{x} \mathrm{Al}_{2}$ [35, 58, 60, 6265], CsCl-type structure SmZn and SmCd [29], and Sm metal [61]. One conclusion to be drawn from these studies is that the magnetic properties of $\mathrm{Sm}^{3+}$ ions in various compounds are influenced to a significant degree by the first excited $J=7 / 2$ states, in addition to the ground $J=5 / 2$ state. There are two reasons for this, 1) the exchange and Zeeman terms along with the crystal field of the surrounding $\mathrm{N}$ ions cause admixtures of the ground and excited 

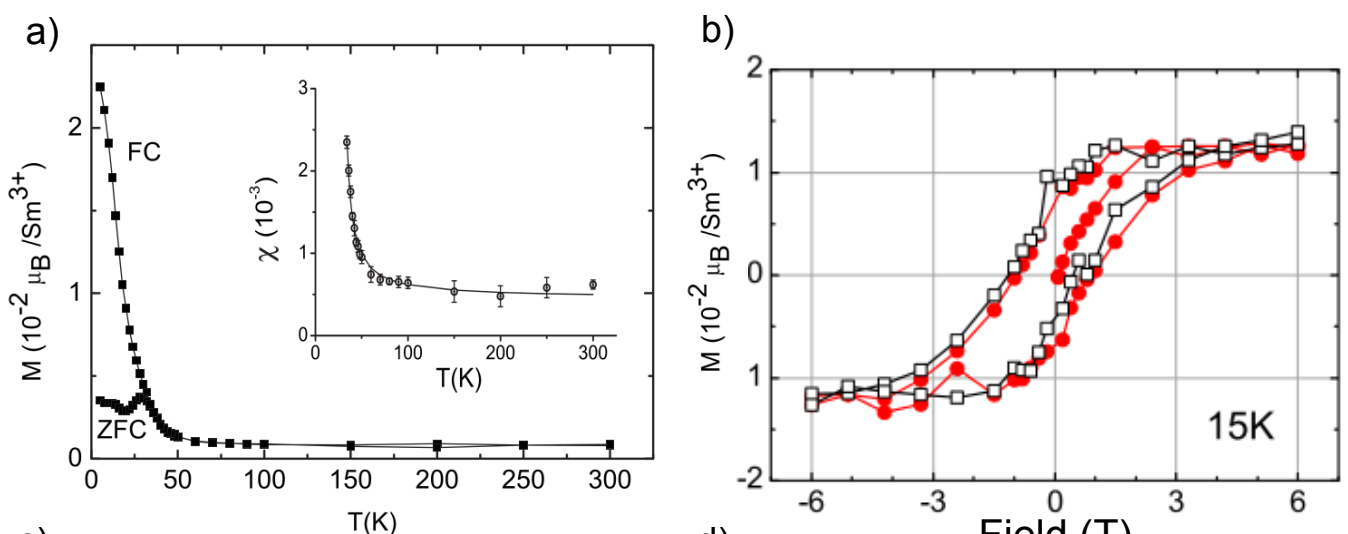

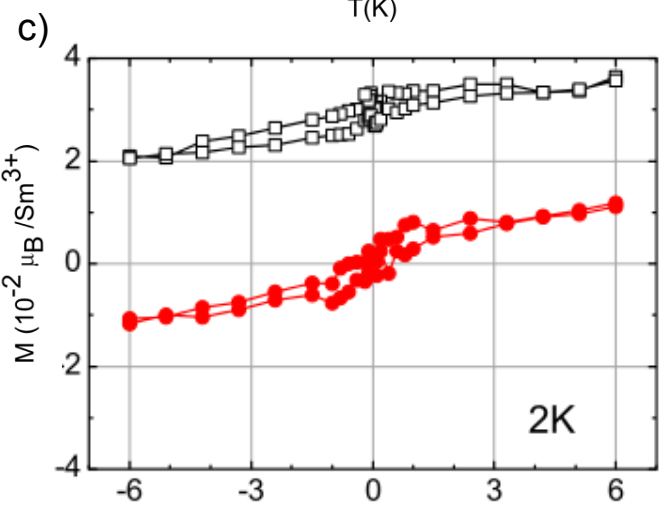

Field $(\mathrm{T})$

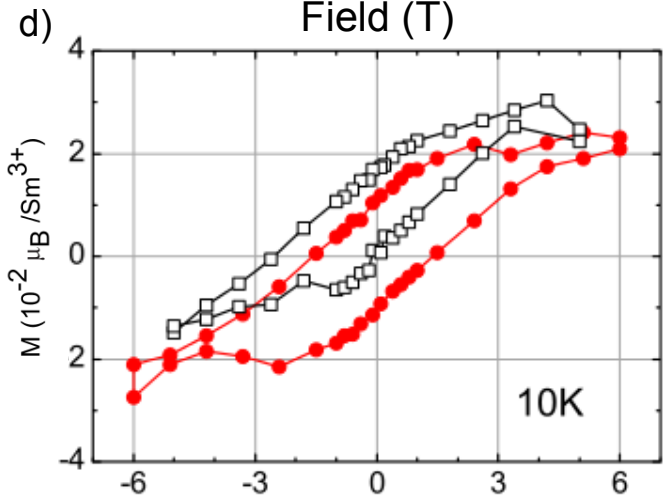

Field $(\mathrm{T})$

Figure 4.1: Plots taken from Meyer et al. [30] showing magnetization measurements of SmN. a) Susceptibility shows a fit to Curie-Weiss with temperature independent van Vleck contribution. b)-d) Hysteresis curves with field cooling in $6 \mathrm{~T}$ (white squares) and with zero field cooling (red circles). The coercive field at $2 \mathrm{~K}$ is greater than $6 \mathrm{~T}$, preventing significant hysteresis, however the shift in the field cooled loop at $2 \mathrm{~K}$ shows that ferromagnetic order is present. 
states, and 2) the small ground state $g$ factor of $2 / 7$ for $\mathrm{Sm}^{3+}$ means the matrix elements within the $J=5 / 2$ state are small compared to matrix elements between the ground and excited states. We shall demonstrate these statements explicitly in the following.

For $\mathrm{SmN}$ we are interested in understanding the origin of the small ferromagnetic moment of about $0.035 \mu_{B}$. Calculations carried out by Larson et al. [14] using density functional theory calculations in the LSDA $+U$ approximation found the same magnitude ferromagnetic moment, however they found that the net moment was parallel to the $4 f$ spin moment, in contradiction to experimental results [19]. The $4 f$ orbital and spin moments were calculated to be $m_{L}^{4 f}=-4.85 \mu_{B}$ and $m_{S}^{4 f}=4.91 \mu_{B}$. Small contributions were also found to the $\operatorname{Sm~} 5 d$ states $\left(0.105 \mu_{B}\right.$ parallel to $\left.m_{S}^{4 f}\right)$ and from the N $2 p$ states $\left(0.136 \mu_{B}\right.$ parallel to $\left.m_{L}^{4 f}\right)$. It is interesting to note that these moments were calculated by assuming a "modified" Hund's rule ground state as a starting point for the calculation ${ }^{2}$, assuming not that $L$ is a maximum (Hund's second rule, as in Chapter 2) but that $L_{z}$ should be a maximum in the ferromagnetic phase. In this case they a priori assume the $z$ components of the $4 f$ moments are at the maximum, and let the calculation run until convergence. However, it is not clear that this is a good starting point. To gain some insight into we turn to the atomic $4 f$ Hamiltonian.

\subsubsection{Calculation procedure}

To calculate the spontaneous ferromagnetic moment of SmN effects, we consider the single ion Hamiltonian

$$
\mathscr{H}=\mathscr{H}_{\mathrm{SO}}+\mathscr{H}_{\mathrm{ex}}+\mathscr{H}_{\mathrm{CF}}
$$

where the terms correspond to contributions from the $4 f$ spin-orbit coupling, exchange coupling, and crystal field interaction. In the $\mathrm{Sm}^{3+}$ ion, the $J=5 / 2$ ground state is separated by about $1500 \mathrm{~K}$ from the first excited $J=7 / 2$ state

\footnotetext{
${ }^{2}$ They found that a guess of the initial state following the modified Hund's rules symmetry yielded a lower energy solution compared to an initial state which obeyed strict cubic symmetry.
} 
$[29,30]$. This seems to be large enough a separation to ignore any contribution from the $J=7 / 2$ state when considering that the ferromagnetic phase of $\mathrm{SmN}$ occurs below $30 \mathrm{~K}$. However, in the $\mathrm{Sm}^{3+}$ ion the inclusion of this state is known to be important $[29,35]$. We begin by examining the simplest case, where there is no external field or crystal field. The Hamiltonian (4.1) including only spinorbit coupling and exchange can then be written as

$$
\mathscr{H}=\Lambda \mathbf{L} \cdot \mathbf{S}+\mathbf{H}_{\mathrm{ex}} \cdot \mathbf{S}
$$

The spin-orbit coupling constant $\Lambda$ is approximately $\Lambda=430 \mathrm{~K}$, calculated from a knowledge of the energy splittings between $J$ multiplets, which is expressed as $\Delta E=E_{J+1}-E_{J}=\Lambda(J+1)$, where $\Delta E=1500 \mathrm{~K},[29,30]$.

The second term represents the effect of inter-ion exchange through an exchange field $\mathbf{H}_{\mathrm{ex}}$ which is defined by $\mathbf{H}_{\mathrm{ex}}=-2 J_{\mathrm{ex}}\left\langle S_{z}\right\rangle$, where $J_{\mathrm{ex}}$ is the exchange energy and $\left\langle S_{z}\right\rangle$ is the expectation value of the spin operator. This is just the mean-field approximation discussed in Chapter 2. We note that while the mean-field approximation is often written with the molecular field proportional to the magnetization, this is only true if there is no orbital moment. The exchange acts only on the spin.

The constant $J_{\mathrm{ex}}=7.9 \mathrm{~K}$ is estimated from the $\mathrm{SmN} \theta_{p} \approx 27 \mathrm{~K}$ using the mean-field approximation (Chapter 2$).{ }^{3}$ However, we note that the meanfield theory we derived in Chapter 2 only considered $\theta_{p}$ in the case of a single multiplet. For $\mathrm{Sm}^{3+}$, there is a temperature independent contribution to the susceptibility $\chi_{V V}$. In this case, the mean-field relationship described in Chapter 2 is modified by the van Vleck susceptibility $\chi_{V V}$ :

$$
\frac{1}{\chi}=\frac{1}{\chi_{0}}-\lambda=\frac{1}{\chi_{C W}+\chi_{V V}}-\lambda,
$$

where $\lambda=(V / N) 2 J_{e x}\left(g_{J}-1\right)^{2} /\left(g_{J} \mu_{B}\right)^{2}$. This changes the relationship $\theta_{p}=$ $2 J_{e x}\left(g_{J}-1\right)^{2} J(J+1) / 3 k_{B}$ slightly, and thus our estimate of $J_{e x}$. Solving for

\footnotetext{
${ }^{3}$ In this chapter Kelvins are the most convenient unit to represent the exchange constant, where $k_{B} 300 \mathrm{~K} \approx 25 \mathrm{meV}$.
} 
$J_{\text {ex }}$ gives

$$
J_{e x}=\frac{3}{2} \frac{k_{B} \theta_{p}}{\left(g_{J}-1\right)^{2} J(J+1)}\left[\frac{1}{1+12 k_{B} \theta_{p} / \Delta E}\right] .
$$

where $\Delta E=1500 \mathrm{~K}$. The term in brackets is the correction due to the van Vleck contribution, which can be viewed as a first order correction to the exchange constant due to the first excited $J$ multiplet. Only for $\mathrm{Sm}$ and Eu is this correction large enough to be considered, and in the case of $\mathrm{Sm}^{3+}$ it gives a correction factor of $\left[1+12 k_{B} \theta_{p} / \Delta E\right]^{-1}=0.83$, yielding $J_{e x}=7.9 \mathrm{~K}$. We note that using the data from Meyer, the estimate of $\theta_{p}$ was within uncertainty equal to $T_{C}$. While the correction here does not significantly change the results of the following calculations, we include it demonstrate how excited $J$-multiplets can influence the exchange constant.

In order to diagonalize $\mathscr{H}$, we need to consider two things; 1) an initial value of $\left\langle S_{z}\right\rangle$, and 2) the basis states to include. The first issue can be addressed by adopting a self-consistent routine where an initial guess of $\left\langle S_{z}\right\rangle$ is made. Then $\mathscr{H}$ can be diagonalized, yielding the energy eigenvalues and eigenstates from which we can then calculate the expectation value $\left\langle S_{z}\right\rangle$. This value is then put back into $\mathscr{H}$ and the process repeated until the calculated expectation value differs from the initial by $\left|\left\langle S_{z}\right\rangle_{\text {out }}-\left\langle S_{z}\right\rangle_{\text {in }}\right|<\epsilon$, where $\epsilon$ is some sufficiently small number. The expectation values can then be used to calculate the total ferromagnetic moment

$$
m=-\mu_{B}\left\langle L_{z}+2 S_{z}\right\rangle .
$$

The next question is which basis states to use. There are two choices, the so-called $J M$-basis or $M_{L} M_{S}$-basis (as discussed in Chapter 2). Both are bases are complete, however we use the $J M$ basis for convenience, primarily because crystal field calculations are simplified in this basis. This is the normal scheme used for rare-earths because $\mathscr{H}_{S O}$ is diagonal in this basis, and is generally much larger than exchange, Zeeman, and crystal field energies. In transition metal systems where the Zeeman contribution is dominant, it is more 
convenient to use the $M_{L} M_{S}$ basis. If we consider only a single $J$ multiplet, the matrix elements of the angular momentum operators can be derived via the Wigner-Eckart theorem [23, 32]:

$$
\begin{aligned}
&\left\langle J^{\prime} M_{J^{\prime}}|\mathbf{L} \cdot \mathbf{S}| J M_{J}\right\rangle=\frac{1}{2}\left[\mathbf{J}^{2}-\left(\mathbf{L}^{2}+\mathbf{S}^{2}\right)\right] \delta_{M_{J^{\prime}}, M_{J}} \\
&=\frac{1}{2}[J(J+1)-(L(L+1)+S(S+1))] \delta_{M_{J^{\prime}}, M_{J}} \\
&\left\langle J^{\prime} M_{J^{\prime}}\left|S_{z}\right| J M_{J}\right\rangle=\left(g_{J}-1\right) M_{J} \delta_{M_{J^{\prime}}, M_{J}} \\
&\left\langle J^{\prime} M_{J^{\prime}}\left|L_{z}\right| J M_{J}\right\rangle=\left(2-g_{J}\right) M_{J} \delta_{M_{J^{\prime}}, M_{J}} \\
&\left\langle J^{\prime} M_{J^{\prime}}\left|L_{z}+2 S_{z}\right| J M_{J}\right\rangle=g_{J} M_{J} \delta_{M_{J^{\prime}}, M_{J}},
\end{aligned}
$$

where the delta functions $\delta_{M_{J}, M_{J^{\prime}}}$ show that the matrix elements are diagonal with respect to $M_{J}$.

We are interested in the ground state ferromagnetic moment as $T \rightarrow 0$, which we can compare with low temperature experimental data. This means we diagonalize $\mathscr{H}$ and calculate expectation values within the ground state only, without any thermal averaging. If we restrict to only the $J=5 / 2$ multiplet, the Hamiltonian (4.5) is already diagonal, and the ground state magnetic moment is simply given by Equation (4.9),

$$
m=-\mu_{B}\left\langle J M_{J}\left|L_{z}+2 S_{z}\right| J M_{J}\right\rangle=-\mu_{B} g_{J} M_{J} \approx 0.714 \mu_{B},
$$

where the ground state is denoted by $J=5 / 2$ and $M_{J}=-5 / 2$. This is just the saturation moment $g_{J} \mu_{B} J$, which is 20 times larger than the experimentally observed $0.035 \mu_{B}$ per ion. This is because we have neglected higher multiplets and crystal field effects. We note that the inclusion of the exchange term has

\footnotetext{
${ }^{4}$ When considering excited $J$ multiplets, there is no simple relationship linking $\left\langle L_{z}+2 S_{z}\right\rangle$ and $\left\langle S_{z}\right\rangle$, and the ratio $\left\langle L_{z}\right\rangle$ and $\left\langle S_{z}\right\rangle$ is no longer fixed, but depends on the parameters of the Hamiltonian.
} 
no effect on the ground state if only a single multiplet is considered.

\subsubsection{Excited states}

If we now include the $J=7 / 2$ and higher multiplets, the Hamiltonian acquires off-diagonal terms. The exchange term, proportional to $S_{z}$, has matrix elements connecting states with $J$ differing by 1 , and with the same $M$ value. In fact, we find that the matrix elements of the total moment $\left\langle S_{z}+2 L_{z}\right\rangle$, within the $J=5 / 2$ multiplet (which are of order $g_{J}$ ) are smaller than the matrix elements connecting the $J=5 / 2$ and $J=7 / 2$ multiplets. We first show how to calculate the matrix elements for arbitrary $J$ using the algebraic techniques originally developed by Racah [66]. In particular we follow the approach of Buschow, Dewijn et al. [35, 58-60], who have shown how to carry out crystal field calculations within arbitrary multiplets (See Appendix A for the derivation). In this approach we can calculate the matrix elements of $S_{z}$ and $L_{z}$ as:

$$
\begin{aligned}
& \left\langle J M\left|S_{z}\right| J^{\prime} M^{\prime}\right\rangle=(-1) J^{J-M+L+S+J^{\prime}+1} \sqrt{(2 J+1)\left(2 J^{\prime}+1\right)} \\
& \quad \times\left(\begin{array}{ccc}
J & 1 & J^{\prime} \\
-M & 0 & M^{\prime}
\end{array}\right)\left\{\begin{array}{ccc}
S & S & 1 \\
J & J^{\prime} & L
\end{array}\right\} \sqrt{S(S+1)(2 S+1)},
\end{aligned}
$$

and

$$
\begin{array}{r}
\left\langle J M\left|L_{z}\right| J^{\prime} M^{\prime}\right\rangle=(-1)^{J-M+L+S+J^{\prime}+1} \sqrt{(2 J+1)\left(2 J^{\prime}+1\right)} \\
\times\left(\begin{array}{ccc}
J & 1 & J^{\prime} \\
-M & 0 & M^{\prime}
\end{array}\right)\left\{\begin{array}{ccc}
L & L & 1 \\
J & J^{\prime} & S
\end{array}\right\} \sqrt{L(L+1)(2 L+1)},
\end{array}
$$

where the term in parenthesis is the Wigner $3 j$ symbol, proportional to a Clebsch-Gordan coefficient:

$$
\left(\begin{array}{ccc}
J_{1} & J_{2} & J_{3} \\
M_{1} & M_{2} & M_{3}
\end{array}\right)=\frac{(-1)^{J_{1}-J_{2}-M_{3}}}{\sqrt{2 J_{3}+1}}\left\langle J_{1} M_{1} J_{2} M_{2} \mid J_{3}-M_{3}\right\rangle .
$$

The term in braces is the Wigner $6 j$ symbol, which is equal to a sum over products of Wigner $3 j$ symbols (see, e.g. Cowan [21] for various properties of 
the Wigner symbols). Values of the symbols can be found in tables [21], however algorithms for calculating them are available, e.g., in the SymPy Wigner package [67], written in Python.

The properties of the $3 j$ and $6 j$ symbols result in the selection rules $\Delta J=$ $J^{\prime}-J=0, \pm 1$ and $M=M^{\prime}$. The matrix representation $S_{z}$ or $L_{z}$ will then be composed of pseudo-diagonal submatrices. For example, if we consider the lowest three multiplets $(J=5 / 2,7 / 2,9 / 2)$ of $\mathrm{Sm}^{3+}$, then the matrix representation of $S_{z}$ has the following structure:

$$
\left[S_{z}\right]_{24 \times 24}=\left[\begin{array}{ccc}
{[J=5 / 2]_{6 \times 6}} & {[\Delta J= \pm 1]_{6 \times 8}} & {[0]_{6 \times 10}} \\
{[\Delta J= \pm 1]_{8 \times 6}} & {[J=7 / 2]_{8 \times 8}} & {[\Delta J= \pm 1]_{8 \times 10}} \\
{[0]_{10 \times 6}} & {[\Delta J= \pm 1]_{10 \times 8}} & {[J=9 / 2]_{10 \times 10}}
\end{array}\right]
$$

where each of the nine submatrices is diagonal with respect to $M$. The submatrices corresponding to the same $J$ are square matrices, while the off diagonal submatrices $(\Delta J= \pm 1)$ involving transitions between multiplets have dimension $(2 J+1) \times\left(2 J^{\prime}+1\right)$. The $\Delta J= \pm 2$ matrix elements are all zero by the properties of the $3 j$ and $6 j$ symbols.

Figure 4.2 shows the sign and magnitude of all the matrix elements of $S_{z}$, $L_{z}$ and $M_{z}$ within the $J=5 / 2,7 / 2,9 / 2$ multiplets. In this figure one can observe the matrix elements of $L_{z}+2 S_{z}$ connecting the $J=5 / 2$ and $J=7 / 2$ states are larger than the matrix elements with the $J=5 / 2$ state. This is due to the small $g$ factor of the $J=5 / 2$ state, and is one reason why incorporating higher multiplets is necessary when dealing with the $\mathrm{Sm}^{3+}$ ion.

Now that we have the tools to evaluate all the matrix elements, we can find the eigenstates and eigenvalues of the Hamiltonian (4.1) in the representation which includes excited states. Like in the previous section, we calculate only the ground state expectation value of $L_{z}+2 S_{z}$. We label the eigenstates of $\mathscr{H}$ as $\left|\psi_{i}\right\rangle$ with associated eigenvalues $E_{i}$, which satisfy

$$
\mathscr{H}\left|\psi_{i}\right\rangle=E_{i}\left|\psi_{i}\right\rangle
$$

These eigenstates can be written as a linear combination of the basis states 

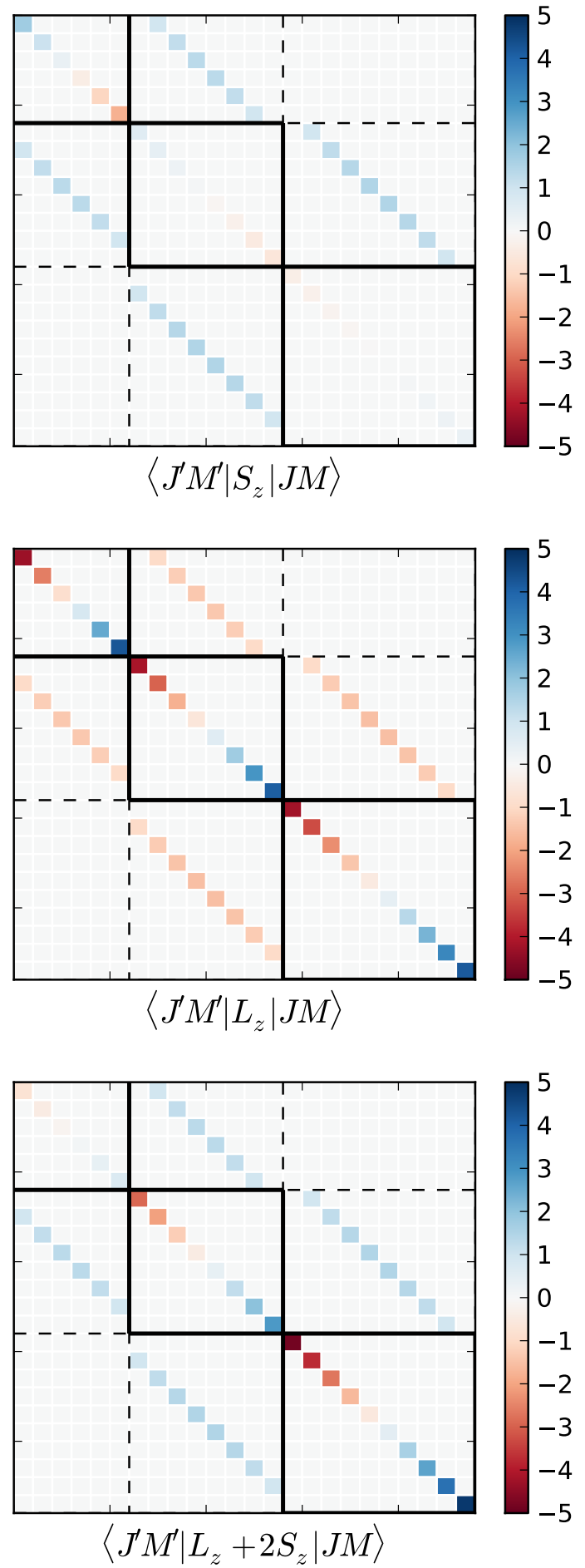

Figure 4.2: The matrix elements of $S_{z}, L_{z}$ and $L_{z}+2 S_{z}$ with for the lowest three multiplets, $J=5 / 2,7 / 2,9 / 2$. 
Chapter 4 SmN and NdN Magnetism

\begin{tabular}{lccc}
\hline$J$ multiplets & $\left\langle S_{z}\right\rangle$ & $\left\langle L_{z}\right\rangle$ & $\left\langle L_{z}+2 S_{z}\right\rangle$ \\
\hline \hline $5 / 2$ & -1.786 & 4.286 & 0.714 \\
$5 / 2,7 / 2$ & -1.852 & 4.352 & 0.648 \\
$5 / 2,7 / 2,9 / 2$ & -1.852 & 4.352 & 0.648 \\
\hline
\end{tabular}

Table 4.1: Expectation values based on number of multiplets included.

$\left|J M_{J}\right\rangle$

$$
\left|\psi_{i}\right\rangle=\sum_{J=5 / 2}^{9 / 2} \sum_{M_{J}=-J}^{J} C_{J, M_{J}}^{i}\left|J M_{J}\right\rangle .
$$

where the $C_{J, M_{J}}^{i}$ are the coefficients found in the diagonalization process. We are interested in the ground state $\left|\psi_{0}\right\rangle$ which has the lowest energy eigenvalue, $E_{0}$. In this case the expectation value of $S_{z}$ can be calculated as

$$
\begin{aligned}
\left\langle\psi_{0}\left|S_{z}\right| \psi_{0}\right\rangle & =\sum_{J=5 / 2}^{9 / 2} \sum_{M_{J}=-J}^{J} \sum_{J^{\prime}=5 / 2}^{9 / 2} \sum_{M_{J^{\prime}}=-J^{\prime}}^{J^{\prime}} C_{J, M_{J}}^{* 0} C_{J^{\prime}, M_{J^{\prime}}}^{0} \\
& \times(-1)^{J-M_{J}+L+S+J^{\prime}+1} \sqrt{(2 J+1)\left(2 J^{\prime}+1\right)} \\
& \times\left(\begin{array}{ccc}
J & 1 & J^{\prime} \\
-M_{J} & 0 & M_{J^{\prime}}
\end{array}\right)\left\{\begin{array}{ccc}
S & S & 1 \\
J & J^{\prime} & L
\end{array}\right\} \sqrt{S(S+1)(2 S+1)} \\
& \times \delta_{J, J^{\prime} \pm 1}, \delta_{M_{J}, M_{J^{\prime}}}
\end{aligned}
$$

The properties of the $3 j$ symbol make the matrix element zero unless $M_{J}=M_{J^{\prime}}$ and $J^{\prime}=J \pm 1$, this has been incorporated explicitly via the delta functions. Calculating the expectation values of $L_{z}$ and $L_{z}+2 S_{z}$ follows similarly.

After carrying out the self-consistent calculation, we find that by including the $J=7 / 2$ and $J=9 / 2$ states in addition to the ground state, we obtain $\left\langle\psi_{0}\left|L_{z}+2 S_{z}\right| \psi_{0}\right\rangle=0.648$, which is about $10 \%$ smaller than the saturation value of 0.714 . This is primarily due to the influence of the $J=7 / 2$ multiplet, as to the quoted precision $\left\langle\psi_{0}\left|L_{z}+2 S_{z}\right| \psi_{0}\right\rangle=0.648$ is returned without inclusion of the $J=9 / 2$ multiplet, as shown in Table 4.1.

We can see that there is some reduction of the ground state magnetic moment due to the exchange interaction mixing in contributions from the 
$J=7 / 2$ multiplet. However, there is no further reduction in the moment by inclusion of the higher multiplets, so a simple exchange mechanism is not enough to explain the small ferromagnetic moment of the SmN. However, we have so far neglected the effects of the crystal field, which are known to be important in rare-earth elements, and which we will explore in the next section.

We note that if the spin orbit coupling is very small, and acts only as a perturbation to the exchange term, then the moment is reduced to zero when higher multiplets are included. This is because for small spin-orbit coupling, $L_{z}$ and $S_{z}$ take on fixed values of $M_{L}$ and $M_{S}$ (i.e $L_{z}|\psi\rangle=M_{L}|\psi\rangle$ and $S_{z}|\psi\rangle=$ $\left.M_{S}|\psi\rangle\right)$, and the ground state then has $M_{L}=5$ and $M_{S}=-5 / 2$, which gives zero net moment. This seems to be equivalent to the modified Hund's rules given in Larson et al[14], where they assume that $\left|L_{z}\right|$ should be a maximum in the ferromagnetic state.

\subsubsection{SmN crystal field}

In this section we incorporate the crystal field terms following the discussion in Chapter 2. The crystal field further mixes the $J=5 / 2$ ground state with the higher multiplets due to off-diagonal terms in the Hamiltonian. We choose the $z$ axis to be along the [111] direction, which is the easy axis if the anisotropy is only due to the single-ion anisotropy. ${ }^{5}$ The crystal field Hamiltonian is given by $[34,35]$

$$
\begin{aligned}
\mathscr{H}_{C F}= & -\frac{2}{3} A_{4} \sum_{i}\left(f_{40}\left(\mathbf{r}_{i}\right)-20 \sqrt{2} f_{43}\left(\mathbf{r}_{i}\right)\right) \\
& +\frac{16}{9} A_{6} \sum_{i}\left(f_{60}\left(\mathbf{r}_{i}\right)+\frac{35 \sqrt{2}}{4} f_{63}\left(\mathbf{r}_{i}\right)+\frac{77}{8} f_{66}\left(\mathbf{r}_{i}\right)\right),
\end{aligned}
$$

where the Tesseral harmonics $f_{k q}\left(\mathbf{r}_{i}\right)$ described in Chapter 2 are tabulated in Hutchings [34], and $A_{4}$ and $A_{5}$ are the crystal field parameters. The sum is over the five $4 f$ electrons. To calculate the matrix elements of $\mathscr{H}_{C F}$ we

\footnotetext{
${ }^{5}$ This was reported in [58] for some Sm systems with similar parameters, and here we also found that choosing $z$ parallel to [001] gave a higher ground state energy. This gave moments differing by no more than $15 \%$, which is not large given experimental uncertainties and the neglect of other contributions to the net moment.
} 
must consider the $\mathbf{r}$-dependence, which means that the radial part of the $4 f$ wave function, which we denote by $\Psi\left(4 f^{n}\right)$, must be included. For example, in Chapter 2 we saw that $f_{k q}\left(\mathbf{r}_{i}\right) \propto r^{k}\left[Y_{k}^{-q}(\theta, \phi)+(-1)^{q} Y_{k}^{q}(\theta, \phi)\right]$, so a typical matrix element to be calculated is given by

$$
\begin{aligned}
& \left\langle\Psi\left(4 f^{n}\right) J M\left|\sum_{i} r_{i}^{k} Y_{k}^{q}\left(\theta_{i}, \phi_{i}\right)\right| \Psi\left(4 f^{n}\right) J^{\prime} M_{J^{\prime}}\right\rangle= \\
& (-1)^{J-M+L+S+J+k} \sqrt{(2 J+1)\left(2 J^{\prime}+1\right)} \times\left(\begin{array}{ccc}
J & k & J^{\prime} \\
-M & q & M^{\prime}
\end{array}\right) \\
& \times\left\{\begin{array}{lll}
L & L & k \\
J & J^{\prime} & S
\end{array}\right\}\left\langle\Psi\left(4 f^{n}\right) L\left\|\sum_{i} r_{i}^{k} Y_{k}\right\| \Psi\left(4 f^{n}\right) L\right\rangle .
\end{aligned}
$$

where the reduced matrix element $R_{k, n}=\left\langle\Psi\left(4 f^{n}\right) L\left\|\sum_{i} r_{i}^{k} Y_{k}\right\| \Psi\left(4 f^{n}\right) L\right\rangle$ is independent of $q$ but does depend on the number $n$ of $4 f$ electrons $(n=5$ for $\left.\mathrm{Sm}^{3+}\right)$. It has been calculated for all of the rare-earths in [35] as $R_{k, n}=$ constant $\times\left\langle r^{k}\right\rangle$. Appendix A discusses the evaluation of $R_{k, n}$ and lists the $R_{k, n}$ values for $\mathrm{Sm}^{3+}$ and $\mathrm{Nd}^{3+}$. The selection rules from the $3 j$ and $6 j$ symbols tell us that $q=M-M^{\prime},|J-J|^{\prime} \leqslant k \leqslant J+J^{\prime}$, and $k \leqslant 2 L$. We are now in a position to calculate the matrix elements of Equation (4.18). However, we have not calculated the radial integrals $\left\langle r^{k}\right\rangle$, and instead factor them out along with the constants $A_{k}$. In this way an arbitrary matrix element of $\mathscr{H}_{C F}$ has the form

$$
\left\langle J M\left|\mathscr{H}_{C F}\right| J^{\prime} M_{J^{\prime}}\right\rangle=a A_{4}\left\langle r^{4}\right\rangle+b A_{6}\left\langle r^{6}\right\rangle,
$$

where $a, b$ are real numbers. By doing this, we leave $A_{4}\left\langle r^{4}\right\rangle$ and $A_{6}\left\langle r^{6}\right\rangle$ being adjustable parameters that don't depend on the validity of the radial integral calculations, which are calculated within various approximations. Our aim is to determine the expectation value of $\left\langle L_{z}+2 S_{z}\right\rangle$ for a range of crystal field parameters $A_{4}\left\langle r^{4}\right\rangle$ and $A_{6}\left\langle r^{6}\right\rangle$.

We now have all the tools to diagonalize the full Hamiltonian using the self-consistent scheme for $\left\langle S_{z}\right\rangle$ :

$$
\mathscr{H}=\Lambda \mathbf{L} \cdot \mathbf{S}-2 J_{e x}\left\langle S_{z}\right\rangle S_{z}+\mathscr{H}_{C F} .
$$




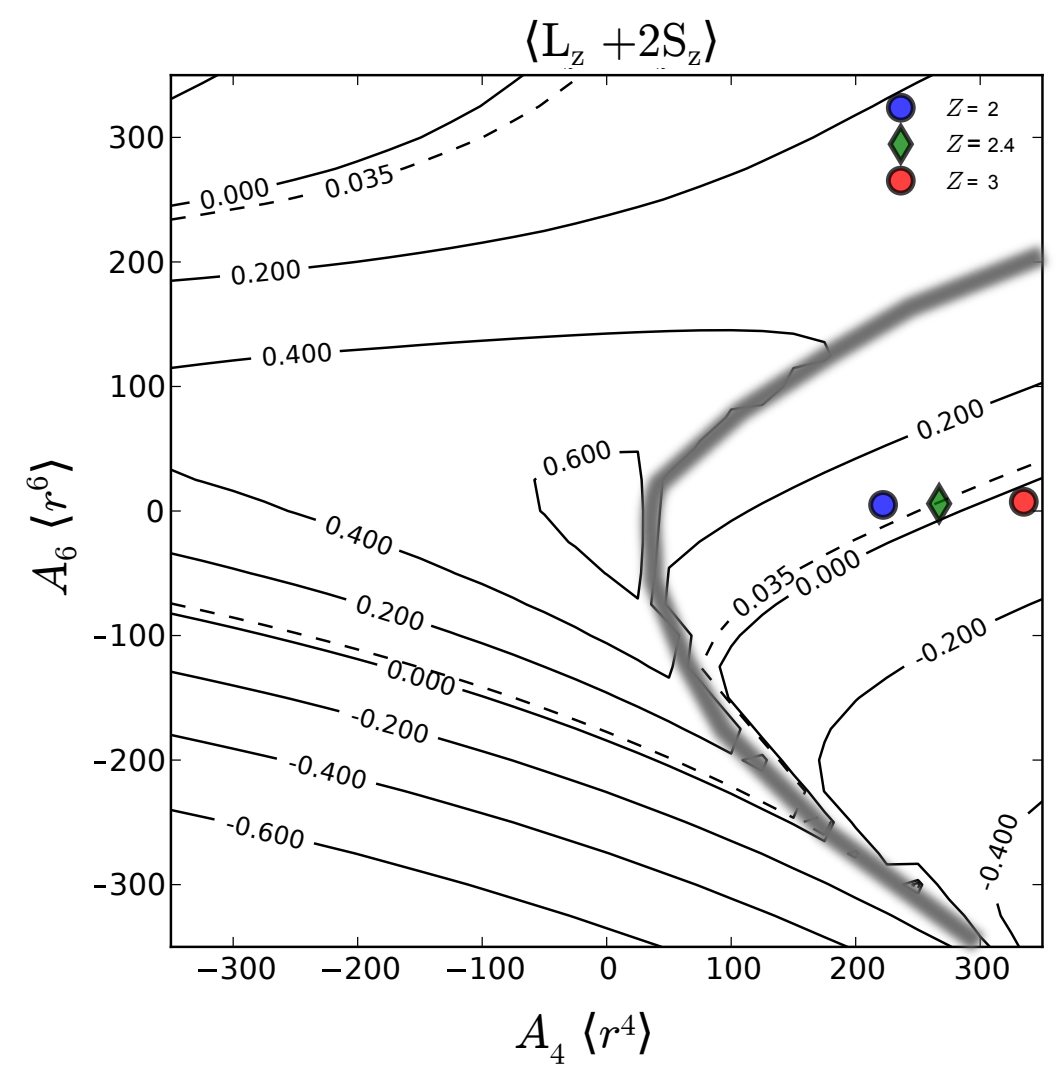

Figure 4.3: Plot of $\left\langle L_{z}+2 S_{z}\right\rangle$ for values of $A_{4}\left\langle r^{4}\right\rangle$ and $A_{6}\left\langle r^{6}\right\rangle$ which includes the three lowest $J$ multiplets. The symbols correspond to the value of $\left\langle L_{z}+2 S_{z}\right\rangle$ calculated using the crystal field parameters derived from a point charge model using an effective charge $Z$. The area to the right of the grey curve corresponds to the $\Gamma_{7}$ ground state.

The result for a parameter range of $A_{4}\left\langle r^{4}\right\rangle, A_{6}\left\langle r^{6}\right\rangle \in[-350 \mathrm{~K},+350 \mathrm{~K}]$ is given in Figure 4.3. We see contours of constant $\left\langle L_{z}+2 S_{z}\right\rangle$ plotted, with the experimental moment of $0.035 \mu_{B}$ shown with a dashed line.

We recall the discussion of Chapter 2, in which we noted that in a cubic crystal field the $J=5 / 2$ ground state splits into a twofold degenerate $\Gamma_{7}$ (doublet) state and fourfold degenerate $\Gamma_{8}$ (quartet) state. Depending on the crystal field parameters, either the doublet or quartet state is lower. By examining the eigenvalues of $\mathscr{H}_{C F}$ it becomes clear that the states to the right of the grey line all have the $\Gamma_{7}$ doublet as the ground state, while to the left of the grey area the quartet is the ground level. 
From the results of Meyer [30] it was determined from the magnitude of the paramagnetic moment, that the $\Gamma_{7}$ doublet has the lower energy in the $J=5 / 2$ manifold. We can thus restrict our attention to this area, and see to return a ferromagnetic moment of $0.035 \mu_{B}$ there is a single line of possible crystal field values. We can compare these values with the point-charge model for comparison.

In the point charge-model, the coefficients $A_{4}$, and $A_{6}$ can be calculated in the following way, assuming that the rare-earth ion has octahedral coordination [68]:

$$
A_{4}=\frac{7}{16} \frac{Z e^{2}}{R^{5}}, \quad A_{6}=\frac{3}{64} \frac{Z e^{2}}{R^{5}}
$$

where $-Z|e|$ is the effective charge of each $\mathrm{N}$ ligand, and $R$ is the separation between the $\mathrm{Sm}$ ion and $\mathrm{N}$. The radial integrals $\left\langle r^{k}\right\rangle$ can be calculated in various approaches. Here we use the non-relativistic Hartree-Fock values from Freeman and Watson [69], with $\left\langle r^{4}\right\rangle=1.897 a_{0}^{4}$ and $\left\langle r^{6}\right\rangle=8.775 a_{0}^{6}$, where $a_{0}=0.529 \AA$ is the Bohr radius. This leads to

$$
A_{4}\left\langle r^{4}\right\rangle=335 \mathrm{~K}, \quad A_{6}\left\langle r^{6}\right\rangle=7.4 \mathrm{~K}, \quad(Z=3)
$$

for $Z=3$, the value we might expect for the trivalent N. From these values one can calculate a moment of $-0.039 \mu_{B}$, which is about the same magnitude as the experimental moment, however here it is negative because the spin moment is larger than the orbital moment. If we calculate for say $Z=2$ the parameters returned are

$$
A_{4}\left\langle r^{4}\right\rangle=222 \mathrm{~K}, \quad A_{6}\left\langle r^{6}\right\rangle=4.9 \mathrm{~K}, \quad(Z=2)
$$

for which a moment of $0.080 \mu_{B}$ is returned, only about twice as large as the experimental moment, but with the correct orbital dominant sign. We find that using $Z=2.4$ gives the crystal field parameters $A_{4}\left\langle r^{4}\right\rangle=267 \mathrm{~K}$ and $A_{6}\left\langle r^{6}\right\rangle=5.9 \mathrm{~K}$ which return the experimental moment. We note that if only the $J=5 / 2$ multiplet is included in the calculation, the moment returned is $0.28 \mu_{B}$, nearly an order of magnitude larger. 


\begin{tabular}{rlccc}
\multicolumn{5}{c}{$J=5 / 2,7 / 2,9 / 2$ included } \\
\hline$J_{e x}$ & $Z$ & $\left\langle S_{z}\right\rangle$ & $\left\langle L_{z}\right\rangle$ & $\left\langle L_{z}+2 S_{z}\right\rangle$ \\
\hline \hline $7.9 \mathrm{~K}$ & 3 & -1.00 & 1.95 & -0.039 \\
$7.9 \mathrm{~K}$ & 2.4 & -0.98 & 2.00 & 0.032 \\
$7.9 \mathrm{~K}$ & 2 & -0.98 & 2.05 & 0.081 \\
\hline \multicolumn{5}{c}{$J=5 / 2$ only } \\
\hline$J_{e x}$ & $Z$ & $\left\langle S_{z}\right\rangle$ & $\left\langle L_{z}\right\rangle$ & $\left\langle L_{z}+2 S_{z}\right\rangle$ \\
\hline \hline $7.9 \mathrm{~K}$ & 2.4 & -0.70 & 1.69 & 0.28 \\
\hline
\end{tabular}

Table 4.2: Expectation values of magnetic moments in $\mathrm{SmN}$ with crystal field parameters calculated in the point charge model, where $-Z|e|$ is the charge of the $\mathrm{N}$ ions.

The parameter $Z$ (or rather $-Z|e|$ ) is essentially an empirical fitting parameter, and a value less than 3, expected for trivalent nitrogen in the ionic limit, indicates that the point charge model breaks down. The view of pointlike charges is clearly an oversimplification as the nitrogen orbitals overlap with the rare-earth valence shell and screening reduces the effective charge felt by the rare-earth ion. Furthermore, calculation of the radial integrals depends on the method used to some degree, with relativistic calculations giving slightly different values.

Nevertheless, while the point charge model cannot explain the crystal field parameters in terms of wave functions, we still see excellent agreement between the experimental moment and the point charge model with $Z$ between 2 and 3. Aside from just the magnitude of the moment, the correct orbital dominant sign is returned. And finally, the $\Gamma_{7}$ doublet ground state observed in the paramagnetic phase is in agreement with the ferromagnetic moment.

We note that another mechanism for the moment reduction is conduction electron polarization, which has a contribution of $m_{C E}=-2 \mathscr{J} \rho\left(E_{f}\right)\left\langle S_{z}\right\rangle \mu_{B}$, where $\mathscr{J}$ is the exchange integral between the $4 f$ and conduction electrons, and $\rho\left(E_{F}\right)$ is the density of states at the Fermi level [29]. Because $m_{C E} \propto m_{S}$, the effect of the conduction electron polarization is to reduce the net moment in the current case. If this contribution is large enough, $m_{S}+m_{C E}$ can even exceed $m_{L}$ and the net moment will be spin-dominant. Because this 
spin-dominance is not observed in $\mathrm{SmN}$, we can conclude that the conduction electron polarization does not play a dominant role, as it does in some metallic Sm systems [29]. This is not surprising as it appears $\mathrm{SmN}$ is not metallic but semiconducting [5], though nitrogen vacancies provide some donor doping. We can make a rough estimate of the contribution of nitrogen vacancies to the magnetic moment. If each vacancy contributes $1-3$ electrons $\left(1 \mu_{B}\right.$ per vacancy), then if $1 \%$ of the $\mathrm{N}$ sites are vacant the contribution to the conduction electron moment is only about $0.01 \mu_{B}$. This $1 \%$ nitrogen vacancy leads to a carrier concentration of about $(0.3-1) \times 10^{21} \mathrm{~cm}^{-3}$ which is common in the RENs, with even lower values found in GdN [12]. This is a much smaller effect than the crystal field even for a large number of vacancies.

The contributions to the net moment from N $2 p$ and Sm $5 d$ states are unknown, due to the inapplicability of the XMCD sum rules at the N K-edge and the rare-earth L-edge. N K-edge XMCD has been observed in GdN for example [3], and is likely present in $\mathrm{SmN}$ as well. The results of Larson indicate that these contributions are around $0.1 \mu_{B}$ (though of opposite sign, cancelling to a large degree).

Band structure calculations are necessary to fully understand the picture in SmN by taking into account the overlap and hybridization effects between the various rare-earth and $\mathrm{N}$ orbitals. However, only two published band-structure calculations are available for $\mathrm{SmN}$, made by Larson et al. [14] and Morari et al. [18] who both used the LSDA $+U$ method. In both cases the calculations found that the spin moment is larger than the orbital moment, and find values $m_{S}^{4 f}$ and $m_{L}^{4 f}$ close to $5 \mu_{B}$. This is at odds with the experimental finding of an orbital dominant moment in $\mathrm{SmN}$ [19], and the present calculations, where the spin moment of $\mathrm{SmN}$ is shown to be antiparallel to the net moment. Furthermore, the current calculations show that both spin and orbital moments have magnitudes close to $2 \mu_{B}$, signalling partial quenching of the moments. This partial quenching is in agreement with recent studies of some heavy rareearth nitrides, ErN, HoN, and DyN [6, 70]. 


\subsection{NdN Magnetism}

$\mathrm{NdN}$, another light rare-earth nitride, is interesting to study alongside $\mathrm{SmN}$ as it also shares an orbital dominant moment. The Hund's rule ground state of $\mathrm{Nd}^{3+}$ is given by $L=6, S=3 / 2$, and $J=9 / 2$. Unlike the near cancellation between spin and orbital moments in ferromagnetic $\mathrm{SmN}$, in NdN the orbital moment should exceed the spin moment considerably, and indeed leads to sizable moments. A handful of magnetic studies on $\mathrm{NdN}$ were carried out in 1960s and 1970s on powder samples, with experimental ferromagnetic moments reported in the range 1.8 to $3.1 \mu_{B}$, along with Curie temperatures of $28-35 \mathrm{~K}$ (see table 33.9 in Ref. [71] and references therein). The $3.1 \mu_{B}$ ferromagnetic moment (from Ref. [72]) should not be taken seriously, as the moment was calculated from neutron diffraction data measured at $80 \mathrm{~K}$ and $290 \mathrm{~K}$, well into the paramagnetic regime where the occupation of crystal field levels is different from the ground state. In particular, an analysis of the magnetization by Schumacher and Wallace [73] found a paramagnetic moment of $3.70 \mu_{B}$ at $296 \mathrm{~K}$, but an ordered moment of $2.15 \mu_{B}$ at $2.5 \mathrm{~K}$. The paramagnetic Curie temperature was found to be $\theta_{p}=15 \mathrm{~K}$, with $T_{C}=35 \mathrm{~K}$ (See Figure 4.4).

For comparison, in the $\operatorname{NdN} J=9 / 2$ ground state, with $g_{J}=8 / 11$, the saturation magnetization is $\mu_{B} g_{J} J=3.27 \mu_{B}$, much larger than any of the reported ordered moments, while the effective paramagnetic moment can be calculated as $\mu_{B} g_{J} \sqrt{J(J+1)}=3.62 \mu_{B}$, close to the value obtained by Schumacher and others [71].

Other Nd pnictides (P, As, Sb, Bi), which order antiferromagnetically, are known to undergo a small tetragonal distortion around the Néel temperature $T_{N}[71,74]$, with the octahedron of $\mathrm{N}$ anions compressed along one axis. There are also a single reference to the same effect occuring in a study of $\mathrm{Nd}_{1-x} \mathrm{O}_{x} \mathrm{~N}_{1-x}$ with $x=0.02 \pm 0.01$. [75]. In this case they also found values of the paramagnetic and ordered moments similar to Schumacher. The indication from the $\mathrm{Nd}$ pnictides is that the structural transition occurs at, or slightly above the magnetic ordering temperature [72, 76, 77].

We present here magnetization measurements on a thin film $\mathrm{NdN}$ sample in order to compare to previous NdN measurements. The MBE method of growth 

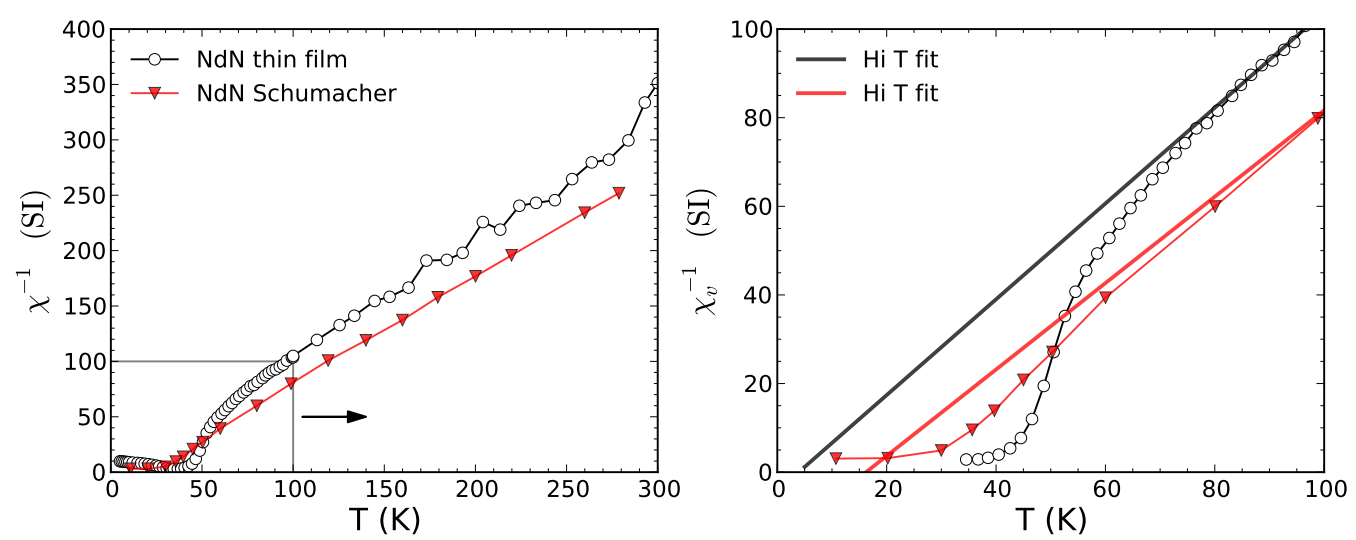

Figure 4.4: Inverse susceptibility of the bulk NdN sample of Schumacher [73], and the thin-film NdN sample, which was measured with $H=250 \mathrm{Oe}$ and in-plane.

was used, outlined in Chapter 3. The NdN film was grown on a $c$-plane sapphire $\left(\mathrm{Al}_{2} \mathrm{O}_{3}\right)$ substrate with an AlN capping layer to prevent oxidation. The film was grown at a rate of $0.1-0.2 \AA / \mathrm{s}$ at $390^{\circ} \mathrm{C}$. The $120 \mathrm{~nm}$ thickness of the film was determined via $\mathrm{x}$-ray reflectivity measurements. While this particular sample was not measured with XRD, similar NdN films grown on sapphire substrates have shown [100] texturing when grown at room temperature while higher temperature growths show an additional [111] reflection.

In Figure 4.4 we present a plot of the inverse susceptibility taken from Ref. [73], along with the recently measured thin film NdN inverse susceptibility for comparison. We have fit the susceptibilities according to a Curie-Weiss approach

$$
\chi=\frac{A}{T-\theta_{p}}+B
$$

with the parameter $A$ given by $n \mu_{0} \mu_{B}^{2} m_{\text {eff }}^{2} / 3 k_{B}$ where $n, m_{\text {eff }}$, and $\theta_{p}$ are the theoretical $\mathrm{Nd}$ ion density, effective moment, and paramagnetic Curie temperature, respectively. The temperature independent contribution for the thin film sample $B$ includes large diamagnetic contributions from both the sapphire substrate and AlN capping layer, which have been removed in Figure 4.4. In this case the thin film data represents $(\chi-B)^{-1}$. This substrate/capping layer 
background signal is the source of the noise in the thin film sample above $100 \mathrm{~K}$. In principle a van Vleck contribution should also be present for NdN, though even at $300 \mathrm{~K}$ it is less than $3 \%$ of the Curie-Weiss contribution.

For the NdN thin film sample, the fit from Equation (4.25) in the region 90-300 K returns the parameters $m_{\mathrm{eff}}=3.5 \pm 0.1 \mu_{B}$, with $\theta_{p}=4 \pm 5 \mathrm{~K}$. This agrees well with the free-ion effective paramagnetic moment of $3.62 \mu_{B}$. The paramagnetic Curie temperature $\theta_{p}$ returned from the fit is however much smaller than the actual ordering temperature $T_{C}$ where the inverse susceptibility intercepts the horizontal axis, at about $42 \mathrm{~K}$. Within the uncertainty limits $\theta_{p}$ is at least a factor of four smaller than $T_{C}$. The Schumacher data, on the other hand, has an effective moment of $m_{\text {eff }}=3.6 \pm 0.1 \mu_{B}$ (which agrees with their quoted value of $3.7 \mu_{B}$ at $296 \mathrm{~K}$.) The high temperature Curie temperature is $\theta_{p}=15 \mathrm{~K}$, with a $T_{C} \approx 30$ to $35 \mathrm{~K}$. Thus $\theta_{p}$ is about a factor of two smaller than the $T_{C}$.

It is interesting to compare the two susceptibilities. In the high temperature regime, the effective moments are about the same within uncertainty, while the values of $\theta_{p}$ differ within the uncertainties between 4-20 K. The lower temperature regime however quite clearly indicates different values of $T_{C}$. The uncertainty in estimates of $\theta_{p}$ make it difficult to conclude much about the different values between samples, so whether $\theta_{p}$ is intrinsically different between samples is not clear.

We can furthermore compare the ordered moments of the samples. The data from Schumacher reports a moment of $2.1 \mu_{B}$ at $2.5 \mathrm{~K}$. To estimate the moment in the thin film sample we turn to Figure 4.5, which shows a hysteresis loop measured at $5 \mathrm{~K}$, with the diamagnetic background subtracted. The high field value corresponds to $0.86 \mu_{B} / \mathrm{Nd}^{3+}$, which is small compared to the theoretical saturation value of $\mu_{B} g_{J} J=3.2 \mu_{B}$. The remanent magnetization is $M_{R}=0.74 \mu_{B}$, which can also be used to estimate the spontaneous moment. If the easy axis lies along one of the high symmetry directions, then in a polycrystalline sample, at remanence the moments will lie distributed within a cone of half-angle $54.7^{\circ}$ around the magnetic field direction. $M_{R}$ is then reduced from the saturation value by about $15 \%$ [78], leading to an estimate of $M_{S}=0.86 \mu_{B}$ for the spontaneous moment, which is in perfect agreement 


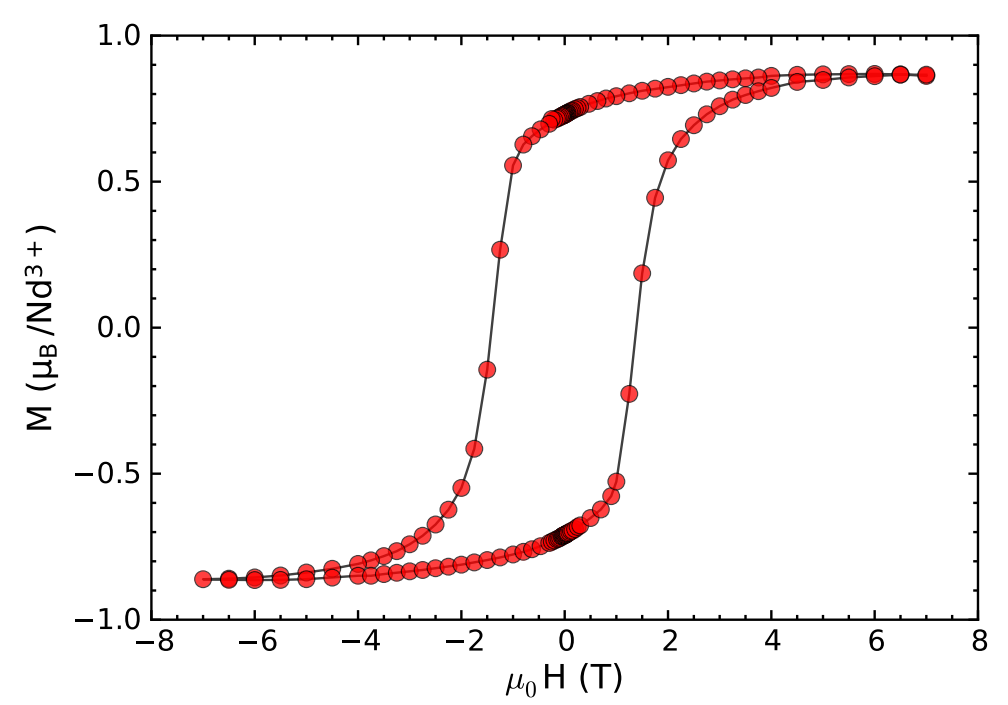

Figure 4.5: Thin-film NdN hysteresis loop measured at $T=5 \mathrm{~K}$ with field in-plane.

with the high field value.

The small ordered moment of the thin-film sample, coupled with the larger $T_{C}$ compared to the data of Schumacher suggest that the ferromagnetic ground state is somehow different between the samples. One possibility is that the thin film is strained due to the substrate, which could change the symmetry of the ground state. Differences in contributions from conduction electrons could play a role as well. Because of the uncertainties in values of $\theta_{p}$ (proportional to the exchange in the mean-field approach), no conclusion can be made as to whether the exchange mechanism is intrinsically different. In the next section we explore the effects of the crystal field on $\mathrm{NdN}$, in an attempt to gain insight into the magnetic properties discussed so far.

\subsubsection{Magnetic moment calculation}

We have already presented the machinery of crystal field calculations in the previous section on $\mathrm{SmN}$, and here we apply the same technique to $\mathrm{NdN}$, with some modifications. We begin by exploring the effects of exchange and additional multiplets. We can estimate the exchange energy from $\theta_{p}$ in the 


\begin{tabular}{lccc}
\hline$J$ multiplets & $\left\langle S_{z}\right\rangle$ & $\left\langle L_{z}\right\rangle$ & $\left\langle L_{z}+2 S_{z}\right\rangle$ \\
\hline \hline $9 / 2$ & -1.23 & 5.73 & 3.27 \\
$9 / 2,11 / 2$ & -1.24 & 5.74 & 3.26 \\
$9 / 2,11 / 2,13 / 2$ & -1.24 & 5.74 & 3.26 \\
\hline
\end{tabular}

Table 4.3: Expectation values based on number of multiplets included

mean-field approximation:

$$
J_{e x}=\frac{3}{2} \frac{k_{B} \theta_{p}}{\left(g_{J}-1\right)^{2} J(J+1)}=8.5 \mathrm{~K} .
$$

for $\theta_{p}=10 \mathrm{~K}$, on the high end of the uncertainty. If we instead use $T_{C}=42 \mathrm{~K}$, the exchange constant is $34 \mathrm{~K}$. We will revisit the estimate of the exchange later. For now, we can estimate the spin and orbital expectation values by diagonalizing $\mathscr{H}=\mathscr{H}_{S O}+\mathscr{H}_{e x}$ with different multiplets. For the $J=9 / 2$ ground state $\mathscr{H}$ is a $10 \times 10$ matrix, while if we include the first excited state, $\mathscr{H}$ grows to a $24 \times 24$ matrix. The results are displayed in Table 4.3. We see that the inclusion of the $J=11 / 2$ multiplet reduces $\left\langle L_{z}+2 S_{z}\right\rangle$ by only $0.3 \%$, and including the $J=13 / 2$ multiplet has even less effect. Of course, the inclusion of crystal field terms may cause more significant mixing between $J$ multiplets. We also note that these values are the same for either exchange parameter used.

\subsubsection{NdN crystal field}

We now consider the crystal field of $\mathrm{NdN}$, and choose the $z$ axis along the [001] direction, ${ }^{6}$ which is given in Refs. [34, 35]:

$$
\mathscr{H}_{\mathrm{CF}}=A_{4} \sum_{i}\left[f_{40}\left(\mathbf{r}_{i}\right)+5 f_{44}\left(\mathbf{r}_{i}\right)\right]+A_{6} \sum_{i}\left[f_{60}\left(\mathbf{r}_{i}\right)-21 f_{64}\left(\mathbf{r}_{i}\right)\right]
$$

In the absence of a Zeeman or crystal field contribution, the $J=9 / 2$ ground state, $\left|\psi_{0}^{9 / 2}\right\rangle$, is ten-fold degenerate, however a cubic crystal field splits the

\footnotetext{
${ }^{6}$ The energy eigenvalues are slightly lower that when calculated in the [111] direction, agreeing with some previous experimental studies finding a [001] easy axis in Nd pnictides [71].
} 

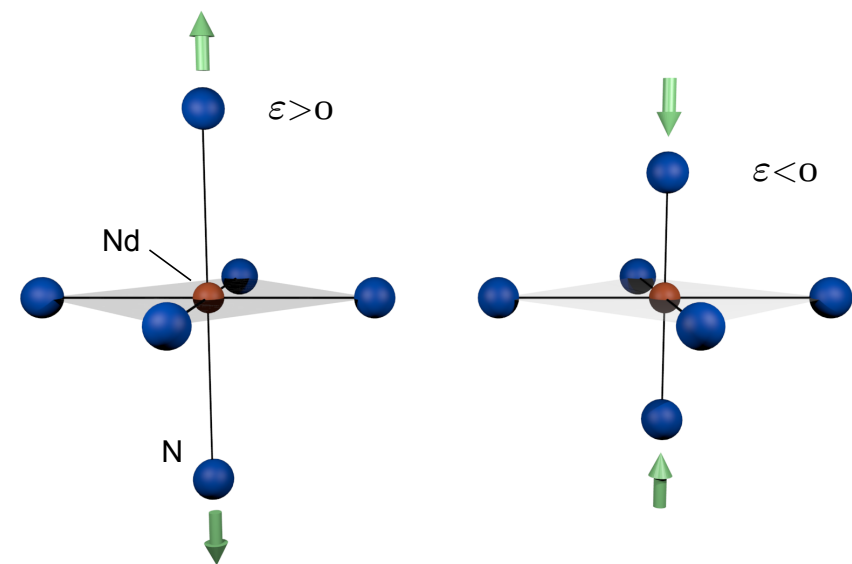

Figure 4.6: A sketch of the tetragonal distortion which occurs in some of the Nd pnictides, though not necessarily NdN. The Nd pnictides have been observed to have a compressive distortion, with $\epsilon<0$.

ground state into three distinct eigenstates of the crystal field Hamiltonian:

$$
\left|\psi_{0}^{9 / 2}\right\rangle \rightarrow\left|\Gamma_{6}\right\rangle+2\left|\Gamma_{8}\right\rangle
$$

where the $\Gamma_{6}$ state is a doublet, and there are two quartets labelled $\Gamma_{8}^{(1)}$ and $\Gamma_{8}^{(2)}$. The ordering of these states depends on the crystal field parameters $A_{4}\left\langle r^{4}\right\rangle$ and $A_{6}\left\langle r^{6}\right\rangle$. There are reports claiming both $\Gamma_{6}$ (Schumacher [79]), and $\Gamma_{8}^{(2)}[71,72]$ ground states in $\mathrm{NdN}$, however the latter was only inferred from $80 \mathrm{~K}$ data and is not a reliable measure of the ordered state.

The tetragonal distortion of some of the rare-earth pnictides, including NdSb was studied via temperature dependent X-ray diffraction by Lévy [74], who also derived the crystal field Hamiltonian for a small tetragonal distortion along one axis. For example Figure 4.6 shows a tetragonal distortion of an octahedrally coordinated ion, where the unit cell is compressed or stretched along one axis. The Hamiltonian for the tetragonal distortion is given by Lévy 
as

$$
\begin{aligned}
\mathscr{H}_{C F}=A_{2} \sum_{i} f_{20}\left(\mathbf{r}_{\mathbf{i}}\right) & +A_{4} \sum_{i}\left[\left(1-\frac{10}{7} \epsilon\right) f_{40}\left(\mathbf{r}_{i}\right)+5 f_{44}\left(\mathbf{r}_{i}\right)\right] \\
& +A_{6} \sum_{i}\left[\left(1-\frac{28}{3} \epsilon\right) f_{60}\left(\mathbf{r}_{i}\right)-21 f_{64}\left(\mathbf{r}_{i}\right)\right] .
\end{aligned}
$$

for a displacement of $\epsilon=\Delta R / R$, where $R$ is the Nd-N separation and $\Delta R$ the distortion. This is nearly the same as the cubic crystal field Hamiltonian, except for the term $\propto A_{2}$ and the factors in parentheses.

We may employ this Hamiltonian, in addition to the cubic version used previously, to investigate the possibility of a tetragonal phase. As there is no data available for the value of $\epsilon$, we estimate it is of similar size as in the other Nd pnictides. For example NdSb has $\epsilon=-0.003$, corresponding to a compression along one axis. For $\mathrm{NdN}_{0.94} \mathrm{O}_{0.06}$ it was found $\epsilon=-0.004$ [75]. For these small displacements, the effect of rescaling the $f_{40}$ and $f_{60}$ is small enough to be ignored: for $\epsilon=-0.003$ the prefactors yield 1.004 and 1.028 for $f_{40}$ and $f_{60}$. The point charge model coefficients $A_{4}$ and $A_{6}$ are calculated as was shown for $\mathrm{SmN}$, while $A_{2}$ in the tetragonal phase is given by

$$
A_{2}=-3 \frac{Z e^{2}}{R^{3}} \epsilon
$$

So for small $\epsilon$ we only need to add the term proportional to $A_{2}$ to the cubic $\mathscr{H}_{C F}$. The radial integrals $\left\langle r^{n}\right\rangle$ for $\mathrm{Nd}^{3+}$ are again taken from [69], with $\left\langle r^{2}\right\rangle=1.001 a_{0}^{2},\left\langle r^{4}\right\rangle=2.401 a_{0}^{4}$, and $\left\langle r^{6}\right\rangle=12.396 a_{0}^{6}$, where $a_{0}$ is the Bohr radius.

We now calculate the ground state ordered moment in either cubic or tetragonal phases, as a function of the crystal field parameters. We begin with the point charge estimates, which were shown to be reasonable for SmN. Table 4.4 shows the results of the calculated ground-state expectation values, calculated within the lowest two multiplets, with varying values of $J_{e x}$ and $Z$, for both cubic and tetragonal crystal fields. The variety of exchange parameters include $J_{e x}=8.5$, estimated from the $\theta_{p}$ value, while $J_{e x}=34 \mathrm{~K}$ is estimated from the large value of $T_{C}$. Other values are shown to see how the 
exchange modifies the ground state moment. If we use $J_{e x}=34 \mathrm{~K}$, the value of $\left\langle L_{z}+2 S_{z}\right\rangle$ is not very sensitive to $Z$ when varied between 2 and 3 . We note that when only the ground state multiplet is considered, the resulting expectation values differ by more than $15 \%$ from the expectation values calculated within the $J=9 / 2$ and $11 / 2$ multiplets. This is because the crystal field in $\mathrm{NdN}$ is large enough to cause significant $J$ mixing. Inclusion of the $J=13 / 2$ multiplet has negligible effect.

The results from the cubic crystal field Hamiltonian in Table 4.4 (a) show that for small exchange constants, a significant reduction in the moments can be found. In particular, for $J_{e x}=12 \mathrm{~K}$ (which corresponds to $\theta_{p} \approx 15 \mathrm{~K}$ ) we find a moment between 2.3 and $2.7 \mu_{B}$, which is reasonably close to the ordered moment of Schumacher, who measured $2.1 \mu_{B}$ [73]. However, the thin-film $\mathrm{NdN}$ ordered moment of $0.86 \mu_{B}$ is not close to any of the values, and only for zero exchange do we begin to approach $1 \mu_{B}$, and this seems an unreasonable exchange constant given the clear indications of ferromagnetism.

For the tetragonal phase, Table 4.4 (b) expectation values for various values of $J_{e x}, Z$, and $\epsilon$ are shown. Positive values of $\epsilon$ denote stretching the unit cell along one axis, while negative values represent compression. We see that small moments close to $0.85 \mu_{B}$ can be obtained only by a stretching of the cell: for $Z=3$ and $\epsilon=0.003$ we obtain good agreement with the ferromagnetic moment. However, this disagrees with the other $\mathrm{Nd}$ pnictides, which have a compression of the unit cell. The negative values of $\epsilon$ tend to only give net moments of over $2.4 \mu_{B}$.

The crystal field parameters used are shown in Table 4.4 (c). To check the reasonableness of these parameters, we extend the calculation of the moments to the paramagnetic phase, where we use the same self-consistent method, except in this case the expectation values must include Boltzmann averaging. We diagonalize the full Hamiltonian now including the Zeeman interaction:

$$
\mathscr{H}=\mathscr{H}_{S O}+\mathscr{H}_{C F}+\mathscr{H}_{e x}+\mathscr{H}_{\text {Zeeman }}
$$


(a) Cubic crystal field

\begin{tabular}{rlccc}
\hline$J_{e x}$ & $Z$ & $\left\langle S_{z}\right\rangle$ & $\left\langle L_{z}\right\rangle$ & $\left\langle L_{z}+2 S_{z}\right\rangle$ \\
\hline \hline $34 \mathrm{~K}$ & 2 & -1.26 & 5.69 & 3.16 \\
$34 \mathrm{~K}$ & 3 & -1.26 & 5.62 & 3.10 \\
\hline $12 \mathrm{~K}$ & 2 & -1.05 & 4.80 & 2.69 \\
$12 \mathrm{~K}$ & 3 & -0.91 & 4.15 & 2.32 \\
\hline $8.5 \mathrm{~K}$ & 2 & -0.88 & 4.03 & 2.27 \\
$8.5 \mathrm{~K}$ & 2.4 & -0.82 & 3.76 & 2.11 \\
$8.5 \mathrm{~K}$ & 3 & -0.75 & 3.44 & 1.93 \\
\hline $10^{-3} \mathrm{~K}$ & 2 & -0.50 & 2.33 & 1.33 \\
$10^{-3} \mathrm{~K}$ & 3 & -0.50 & 2.33 & 1.33 \\
\hline
\end{tabular}

(b) Tetragonal crystal field

\begin{tabular}{rccccc}
\hline$J_{e x}$ & $Z$ & $\epsilon \times 10^{3}$ & $\left\langle S_{z}\right\rangle$ & $\left\langle L_{z}\right\rangle$ & $\left\langle L_{z}+2 S_{z}\right\rangle$ \\
\hline \hline $34 \mathrm{~K}$ & 3 & 3 & -1.21 & 5.41 & 2.99 \\
$34 \mathrm{~K}$ & 3 & -3 & -1.13 & 5.09 & 2.83 \\
\hline $8.5 \mathrm{~K}$ & 2 & 1 & -0.66 & 3.04 & 1.72 \\
$8.5 \mathrm{~K}$ & 3 & 1 & -0.53 & 2.45 & 1.39 \\
& & & & & \\
$8.5 \mathrm{~K}$ & 2 & 3 & -0.31 & 1.48 & 0.85 \\
$8.5 \mathrm{~K}$ & 3 & 3 & -0.26 & 1.26 & 0.74 \\
& & & & & \\
$8.5 \mathrm{~K}$ & 3 & -1 & -0.94 & 4.27 & 2.39 \\
$8.5 \mathrm{~K}$ & 3 & -3 & -1.13 & 5.08 & 2.83 \\
$8.5 \mathrm{~K}$ & 3 & -5 & -1.22 & 5.49 & 3.04 \\
\hline
\end{tabular}

(c) Point-charge crystal field parameters for $\mathrm{NdN}$

\begin{tabular}{cccc}
\hline $\mathrm{Z}$ & $A_{2}(\mathrm{~K})$ & $A_{4}(\mathrm{~K})$ & $A_{6}(\mathrm{~K})$ \\
\hline \hline 2 & 49.9 & 247.1 & 5.8 \\
2.4 & 59.8 & 296.5 & 7.0 \\
3 & 74.8 & 370.6 & 8.7 \\
\hline
\end{tabular}

Table 4.4: Ground state expectation values for cubic (a) and tetragonal (b) crystal fields, with varying effective charge $Z$, and with varying tetragonal distortion parameter $\epsilon$. The two lowest $J$ multiplets were included. (c) The crystal field parameters $A_{k}$ are given for $\mathrm{NdN}$ in the point-charge model for various values of $Z$. 
and calculate the expectation values as

$$
m=-\mu_{B}\left\langle L_{z}+2 S_{z}\right\rangle_{T}=-\mu_{B} \frac{\sum_{n}\left\langle\psi_{n}\left|L_{z}+2 S_{z}\right| \psi_{n}\right\rangle e^{-\beta E_{n}}}{\sum_{n} E_{n} e^{-\beta E_{n}}},
$$

where $\left|\psi_{n}\right\rangle$ are eigenstates of the full Hamiltonian in Equation (4.31). The susceptibility can then be calculated as $\chi=m n / H$, where $n=4 / a^{3}$ is the $\mathrm{Nd}$ ion density determined from the lattice constant $a$. The results are shown in Figure 4.7 alongside the experimental susceptibilities. Very good agreement is found between the Schumacher data and the calculated susceptibility when using $J_{e x}=12 \mathrm{~K}$ and the cubic crystal field parameters calculated with $Z=3$. The thin film NdN sample does not agree with the tetragonal crystal field parameters with $\epsilon=0.003, J_{e x}=8.5 \mathrm{~K}$ and crystal field parameters calculated using $Z=2$, which returned a moment of $0.85 \mu_{B}$ in the ordered phase. No satisfactory agreement for the $\mathrm{NdN}$ thin film susceptibility could be found after exhausting all reasonable combinations of cubic and tetragonal crystal field parameters and $J_{e x}$.

We note that the tetragonal phase is not expected to persist in above the magnetic transition, so it is not surprising to find no agreement when the susceptibility is calculated with tetragonal parameters. More puzzling is the lack of agreement of the thin-film data with the cubic crystal field calculations. This suggests the possibility of lattice strain in both phases which modifies the crystal field below and above $T_{C}$. A [100] textured film might be expected to have a tetragonal distortion, while a film with [111] texturing could have an orthorhombic distortion due to the lattice mismatch with the substrate which is $24 \%$ for $c$-plane sapphire. More structural data is thus necessary to carry detailed calculations which include the correct crystal field symmetry.

We note that the experimental moments are much less than the theoretical saturation value of $\mu_{B} g J=3.2 \mu_{B}$ and much less than the moments from the LSDA $+U$ calculations of Larson et al. [14], which give a net moment of $m=2.9 \mu_{B}$, with $m_{L}^{4 f}=5.88 \mu_{B}$, and $m_{S}^{4 f}=-2.96$. The calculated moment best agreeing with the Schumacher data in the ferromagnetic and paramagnetic phases gives a net moment of $2.3 \mu_{B}$, with $m_{S}=-1.8 \mu_{B}$ and $m_{L}=4.15 \mu_{B}$ which indicates that partial quenching takes place. 


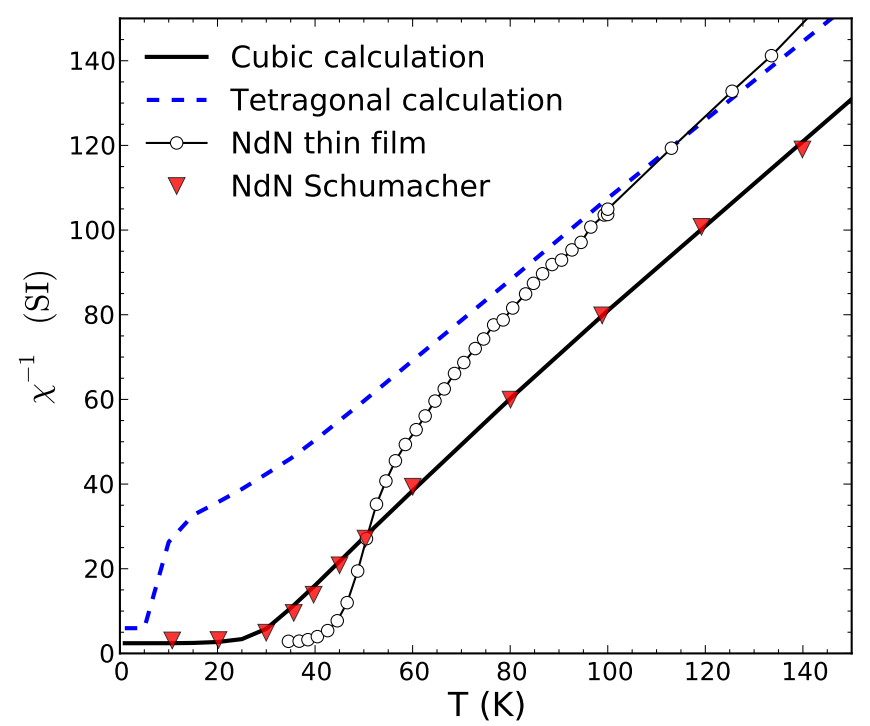

Figure 4.7: NdN Susceptibility. Cubic crystal field calculation parameters: $J_{e x}=12 \mathrm{~K}, Z=3$. Tetragonal crystal field calculation parameters: $J_{e x}=8.5$, $Z=2$.

We then conclude that the thin film sample shows quite distinct magnetic properties as compared to the bulk data of Schumacher et al. [73] and cannot be interpreted within cubic or tetragonal crystal field models. The Schumacher data fit very well to calculations within a cubic crystal field using the exchange parameter estimated from $\theta_{p}$ and point charge calculations for $Z=3$. The thin film then is likely strained, presumably due to the $c$-plane $\mathrm{Al}_{2} \mathrm{O}_{3}$ substrate. It is usually found that the RENs grow with [111] texturing on hexagonal substrates [2], in this case the substrate has a lattice constant of $4.79 \AA$ versus the Re-Re separation of $3.76 \AA$, leading to a large lattice mismatch. In the case of [111] oriented $\mathrm{NdN}$, it is possible that the substrate could introduce an orthorhombic distortion. In that case the crystal field would have to be modified, and could possibly explain the thin film magnetic data. Information on the low temperature lattice constant and any distortions would allow for calculation of these effects, though the cubic field Hamiltonian would have to be generalized.

Furthermore, the contribution from conduction electrons due to nitrogen vacancies could play a role, as it is likely the samples have different carrier 
concentrations. It is doubtful that conduction electrons alone could explain the difference in the ground state ordered moment, as to account for a $1 \mu_{B}$ smaller moment in the thin film would require a high carrier concentration (and metallic conductivity), while the resistivity data from this sample suggest doped semiconductor behavior with a resistivity of $0.6 \mathrm{~m} \Omega$-cm at $300 \mathrm{~K}$ increasing to $1 \mathrm{~m} \Omega-\mathrm{cm}$ at low temperature. We however note that GdN has been shown to have a $T_{C}$ of $50 \mathrm{~K}$ for low carrier concentrations, increasing to $70 \mathrm{~K}$ for carrier concentrations above $\approx 10^{20} \mathrm{~cm}^{-3}$. This enhancement in $T_{C}$ was shown to be due to the formation of magnetic polarons at nitrogen vacancies, as opposed to an RKKY-type effect $[12,13]$. A similar magnetic polaron scenario in $\mathrm{NdN}$ might explain the rather large $T_{C}$ in the thin film sample compared to the Schumacher data, however more detailed studies will need to be carried out.

\subsubsection{Effects of the crystal field on $T_{C}$}

Finally, we conclude the chapter with a discussion of the exchange parameter $J_{e x}$. It is not a priori clear how the crystal field affects the relationships between the ordering temperature, $T_{C}$, the high temperature paramagnetic Curie temperature $\theta_{p}$ and exchange parameter $J_{e x}$. In order to clarify the situation, and justify the estimation of $J_{e x}$ from $\theta_{p}$, we again examine the paramagnetic regime within the crystal field theory.

We are mostly interested in a qualitative understanding, and so carry out an analysis of the susceptibility in second order perturbation theory (see, e.g. $[27,32])$ where we treat the Zeeman term as a perturbation to the spin-orbit and crystal field Hamiltonian. The exchange interaction is then incorporated in a self-consistent mean-field approach, as described in Chapter 2. We can simplify by only considering the ground state $J$ multiplet $^{7}$, noting that this is not strictly justified, as the $J=11 / 2$ multiplet had a significant effect on ground state ferromagnetic moment.

\footnotetext{
${ }^{7}$ The second order susceptibility for arbitrary multiplets is described in [35, 61], though its complexity makes direct diagonalization of the full Hamiltonian a better option.
} 
In the zeroth order, the Hamiltonian has eigenstates $\left|n_{k}\right\rangle$ that obey

$$
\left(\mathscr{H}_{S O}+\mathscr{H}_{C F}\right)\left|n_{k}\right\rangle=E_{n}\left|n_{k}\right\rangle
$$

where $n$ indexes the energy level $E_{n}$ and $k$ indexes the various degenerate states sharing the same $E_{n}$ (for example the $\left|n_{k}\right\rangle$ could be the doubly degenerate $\left|\Gamma_{6}\right\rangle$ state). We now use these eigenstates to calculate the susceptibility in the absence of any exchange interaction, denoted $\chi_{0}$. This is given by $[27,80]$ :

$\chi_{0}=\frac{N}{V} \sum_{n}\left(\frac{g^{2} \mu_{B}^{2}}{k_{B} T} \sum_{k, k^{\prime}}\left|\left\langle n_{k}\left|J_{z}\right| n_{k^{\prime}}\right\rangle\right|^{2}+2 \mu_{B}^{2} \sum_{m \neq n} \sum_{k, k^{\prime}} \frac{\left|\left\langle n_{k}\left|J_{z}\right| m_{k^{\prime}}\right\rangle\right|^{2}}{E_{m}-E_{n}}\right) \frac{e^{-\beta E_{n}}}{\mathcal{Z}}$,

where $\mathcal{Z}=\sum_{n} \omega_{n} e^{-\beta E_{n}}$ is the partition function, and $\omega_{n}$ is the degeneracy of the eigenstate $\left|n_{k}\right\rangle$. Here $J_{z}$ is the total angular momentum operator, and $E_{n}$ are the unperturbed energies found in Equation 4.33. The first term is the $T^{-1}$ "Curie" term, while the second term is sometimes called the van Vleck contribution, though it is not the same as the van Vleck contribution from the higher multiplets. If the temperature is low enough, then only the ground state will be occupied and only the first term contributes. However, if there is a close by excited state and the separation $\Delta=E_{n}-E_{m}$ is small, then the second term becomes relevant. In the analysis of the ferromagnetic moment of NdN above, in the cubic crystal field the separation between the ground and first excited crystal field states is $\Delta \approx 40 \mathrm{~K}$, depending on the parameters. Therefore in the paramagnetic phase we must consider both states when calculating $\chi$. If we assume only these two lowest states contribute at low temperatures (less than $100 \mathrm{~K}$ ) then the susceptibility simplifies to

$$
\begin{array}{r}
\chi_{0}=\frac{N}{V} \frac{g^{2} \mu_{B}^{2}}{\omega_{0}+\omega_{1} e^{-\beta \Delta}}\left(\frac{1}{k_{B} T} \sum_{k, k^{\prime}}\left(\left|\left\langle J_{z}\right\rangle_{00}^{k k^{\prime}}\right|^{2}+\left|\left\langle J_{z}\right\rangle_{11}^{k k^{\prime}}\right|^{2} e^{-\beta \Delta}\right)\right. \\
\left.+2 \sum_{k, k^{\prime}} \frac{\left|\left\langle J_{z}\right\rangle_{01}^{k k^{\prime}}\right|^{2}}{\Delta}\left(1-e^{-\beta \Delta}\right)\right),
\end{array}
$$

where we have used the notation $\left\langle J_{z}\right\rangle_{n m}^{k k^{\prime}} \equiv\left\langle n_{k}\left|J_{z}\right| m_{k^{\prime}}\right\rangle$ and $\Delta=E_{1}-E_{0}$. 
This has the form

$$
\chi_{0}=\frac{A(T)}{T}+B(T)
$$

where $A(T)$ and $B(T)$ vary exponentially with temperature. The susceptibility in the presence of exchange $\chi$ can be incorporated now by using the selfconsistent mean-field approximation, as was done in Chapter 2:

$$
\chi=\frac{\chi_{0}}{1-\lambda \chi_{0}} \quad \text { where } \quad \lambda=2 J_{e x} \frac{(g-1)^{2}}{(N / V)\left(g \mu_{B}\right)^{2}} .
$$

We can now write $\chi$ in a form which incorporates exchange and crystal field effects:

$$
\chi=\frac{\bar{A}+\bar{B} T}{T-\lambda \bar{A}}
$$

where $\bar{A}=A(T) /(1-\lambda B(T))$ and $\bar{B}=B(T) /(1-\lambda B(T))$. We can check that in the limit $B(T) \rightarrow 0$ (equivalent to a large separation $\Delta$ between levels, or $T \rightarrow 0)$ we arrive at Curie-Weiss behavior:

$$
\chi=\frac{A(0)}{T-\lambda A(0)} . \quad \text { for } \Delta \rightarrow \infty
$$

This limit is not useful for NdN however, since the ordering point, $35 \mathrm{~K}$ to $42 \mathrm{~K}$, is roughly equal to the splitting between the ground and first excited states. Returning to Equation 4.38, we see that the exchange parameter and crystal field modify both $T_{C}$ and the effective moment. The susceptibility can be seen to diverge at an effective ordering temperature

$$
T_{C}^{\star}=\lambda \bar{A}=\frac{\lambda A(T)}{1-\lambda B(T)} .
$$

So it is clear that the ordering temperature depends not only on the exchange, via the molecular field $\lambda$, but also on the crystal field through both $A(T)$ and $B(T)$ terms. Figure 4.8 shows $T_{C}^{\star}$ calculated with point charge parameters for $Z=3$ and $J_{e x}=12.5 \mathrm{~K}$. This value of $J_{e x}$ roughly corresponds to Schumacher's value of $\theta_{p}=15 \mathrm{~K}$. When $T_{C}^{\star}=T$, the maximum value of $T_{C}^{\star}$ is reached; $T_{C}^{\star}>$ 


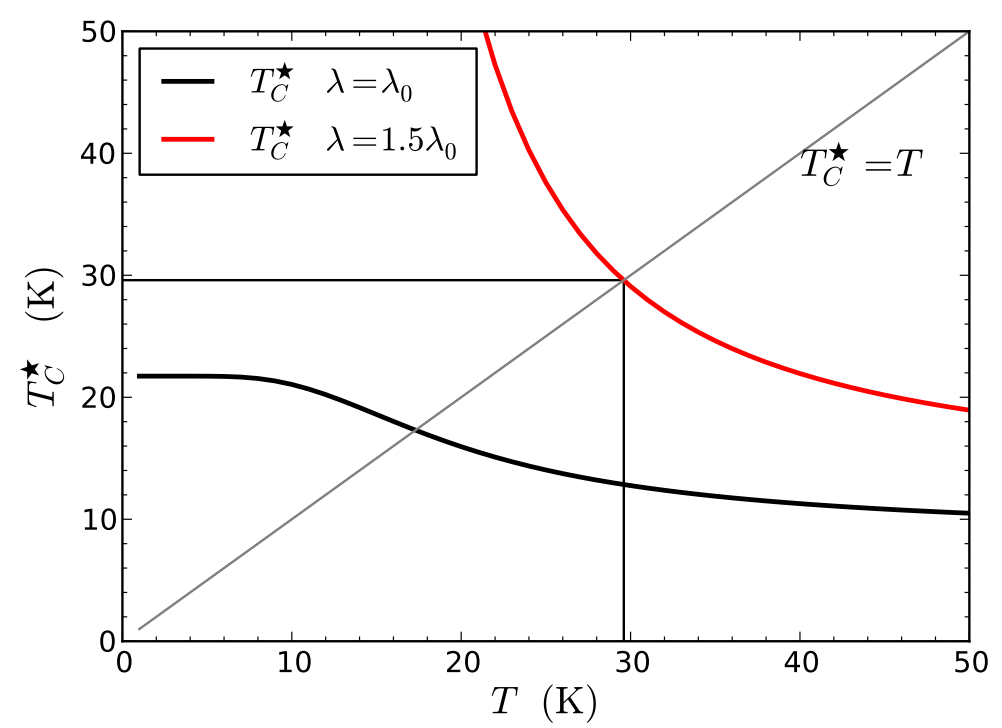

Figure 4.8: $T_{C}^{\star}$ calculated with $J_{e x}=12.5 \mathrm{~K}$ and a cubic crystal field using point charge parameters for $Z=3$. $\lambda_{0}$ corresponds to the molecular field constant for the ground state multiplet $\lambda_{0}=(N / V) 2 J_{e x}(g-1)^{2} /\left(g^{2} \mu_{B}^{2}\right)$.

$T$ has no physical significance. Using $\lambda$ for the ground state multiplet (Eq 4.37), we see that maximum value of $T^{\star}$ is only about $17 \mathrm{~K}$, just slightly increased from $\theta_{p}$. However, we have neglected the effects of excited multiplets, and so the disagreement with the Schumacher data is not surprising. If we try to account for this by scaling $\lambda$ by 1.5, we good agreement with the experimental $T_{C}$. We note that the full calculation of $\chi$, shown in Figure 4.7 reproduces the difference in $\theta_{p}$ and $T_{C}$ very well.

To summarize, Figure 4.8 illustrates that as the occupation of the crystal field changes with temperature, for the given parameters, the ordering temperature increases from that expected from exchange only. This may change dramatically depending on the separation between crystal field states and the size of the matrix elements. We can conclude that when considering the size of the exchange constant, $J_{e x}$, one should estimate it from the high temperature susceptibility. In fact, this is not surprising, as Curie's law is derived under the assumption that the temperature is large enough that all of the $M_{J}$ levels within a $J$ multiplet are occupied. At lower temperatures, the occupation 
changes due to splitting of the crystal field, and the factor $(g-1)^{2} J(J+1)$ does not reflect the actual spin contribution to the moment. Thus using the actual ordering temperature to estimate $J_{e x}$ overestimates the exchange, in some cases by more than a factor of two, as for NdN. A Similar effect can be seen in ErN [6], though it is smaller than in NdN.

\subsection{Conclusion}

In conclusion, we have shown that the small $\mathrm{SmN}$ moment is very well explained by the incorporation of higher multiplets and crystal field with parameters estimated within the point-charge model. The $\Gamma_{7}$ ground state is in accord with a calculation of the effective paramagnetic moment if only the $\Gamma_{7}$ state is occupied, as demonstrated in Meyer et al[30].

We also found that the bulk NdN data of Schumacher was well described by a cubic crystal field calculation of both the ground state ordered moment and paramagnetic susceptibility. The thin-film NdN sample could not be adequately explained within a cubic or tetragonal crystal field however. The indication is that strain may play an important role in the thin film, modifying the crystal field in some way. Nitrogen vacancies may also play a role in producing different values of $T_{C}$ and ordered moments in the samples. The exchange energy was shown to be best estimated from the high temperature susceptibility, and the ordering temperature was shown to have a strong crystal field dependence.

Finally, we note that the resulting spin and orbital moments calculated for $\mathrm{SmN}$ and $\mathrm{NdN}$ are partially quenched, in agreement with experimental data, and in contrast to LSDA $+U$ calculations $[14,18]$ which found nearly maximum values of $m_{L}^{4 f}$ and $m_{S}^{4 f}$. Recent neutron reflectivity experiments on DyN,ErN, and $\mathrm{HoN}$ found much smaller ordered moments at $5 \mathrm{~K}$ than the free ion $\mu_{B} g_{J} J$ values [70]. The authors pointed out the disagreement with the LSDA $+U$ calculations and proposed that the ordered moments could be explained by a fully quenched $(L=0)$ ground state moment, however full quenching of $L$ is unlikely. It is more likely that partial quenching of the orbital moment occurs, and via the spin-orbit coupling the spin moment $m_{S}$ is quenched to a similar 


\subsection{Conclusion}

degree, which is exactly what the crystal field calculations predict. 
Chapter 4 SmN and NdN Magnetism 


\section{Chapter 5}

\section{Twisted Magnetization in SmN/GdN}

\subsection{Introduction}

As we have seen for $\mathrm{SmN}$ and $\mathrm{NdN}$, the strong spin-orbit interaction of the $4 f$ shell results in a dominant orbital contribution to the ferromagnetic moment. This is in contrast to more familiar transition metal systems, where the orbital moment is usually quenched. In this chapter we show how this orbital-dominant feature facilitates the fabrication of a novel class of magnetic heterostructures. In particular we describe an investigation of interfaces between GdN and SmN using XMCD.

Twisted, or inhomogeneous ordering of the magnetization occurs in some magnetic systems due to competing interactions which favor opposing alignments of the magnetization. Competing interactions may arise from a number of sources, including surface or bulk anisotropies, interface exchange, Zeeman coupling, or chiral interactions [81, 82]. These twisted phases can be viewed as types of engineered domain walls, and thus have important implications for spintronics applications, where current-driven domain wall motion is an active area of research [83-86]. So far, twisted phases are known to manifest in diverse magnetic systems [38, 81, 82, 87-91], however these all fall under the conventional spin-dominant paradigm of magnetism where the orbital moment plays no significant role. Competing interactions in the presence of a dominant 
a)

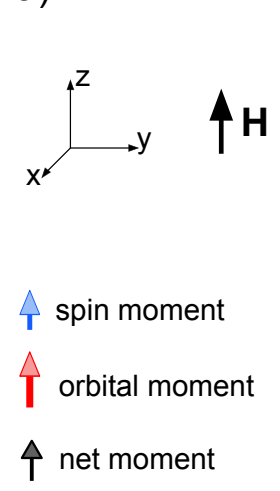

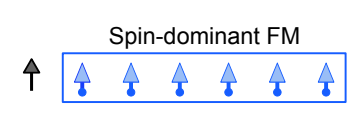

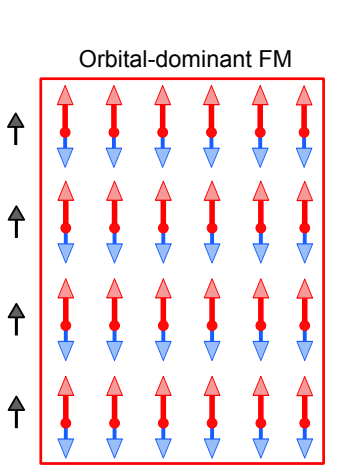

b)

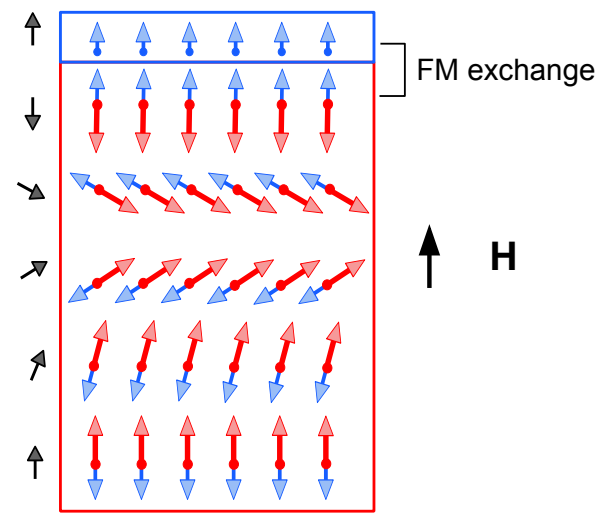

Figure 5.1: (a) A sketch of a single atomic layer of a spin-dominant ferromagnet (e.g. GdN) and a cross section of multiple atomic planes of an orbitaldominant $(\mathrm{SmN})$ ferromagnet. (b) Cross-section of an interface between spin and orbital-dominant ferromagnets. A twisted phase develops in the orbitaldominant magnet due to exchange-Zeeman competition which occurs if the spin-dominant layer remains fixed due to its large Zeeman coupling.

orbital moment have so far remained unexplored, yet the opportunity exists within the rare-earth nitride series, where orbital-dominant magnetism is possible in the first half of the series due to strong spin-orbit coupling of the $4 f$ electrons.

GdN, with the $\mathrm{Gd}^{3+}$ ion, has a half-filled $4 f$ shell, for which Hund's rules state that the seven electrons fill all of the orbital states with spin-up electrons; $L=0$ and $S=J=7 / 2$. It thus has a purely spin moment of $7 \mu_{B}$. The indirect exchange interaction aligns the spins below a Curie temperature of about $50 \mathrm{~K}$, rising to $70 \mathrm{~K}$ under heavy donor doping [12], but the spherical symmetry of the $L=0$ shell interacts very weakly with the crystalline environment, leading to a coercive field smaller than 100 Oe.[92]

This is in stark contrast to $\mathrm{SmN}$, which is not only orbital-dominant with a near-zero moment, but has a coercive field of over $6 \mathrm{~T}$ at $2 \mathrm{~K}$ due to the non-spherical orbital wave function and weak Zeeman interaction associated with the small magnetic moment.

In the following we exploit the contrasting properties of $\mathrm{GdN}$ and $\mathrm{SmN}$ in $\mathrm{SmN} / \mathrm{GdN}$ thin film heterostructures, and observe a twisted phase arising from 
a novel competition between spin and orbital magnetism. The spin-dominant GdN is fixed parallel to an external magnetic field, and its much larger Zeeman interaction ensures that it provides a rigid layer which pins the $\mathrm{SmN}$ spin at the SmN-GdN interface. The pinning of the SmN, with its 200-fold weaker Zeeman coupling, takes place through ferromagnetic exchange coupling with the GdN, resulting in a $\mathrm{SmN}$ spin-moment parallel to that of the GdN, while the orbital-moment is antiparallel. This interface pinning is opposed by the orbital-dominant Zeeman alignment of the bulk SmN, which tends to align the $\mathrm{SmN}$ magnetization in the opposite sense, and thus drives the rotation of the magnetization across the $\mathrm{SmN}$ layer. Figure 5.1 sketches the effects of exchange coupling between spin and orbital dominant ferromagnets.

It is important to note that the $\mathrm{GdN} / \mathrm{SmN}$ system is fundamentally different from the conventional spin-dominant ferromagnetic systems displaying twisted phases, as was mentioned in Chapter 2. The most common exchange spring systems, composed of hard and soft ferromagnetic layers, are first magnetized in one direction, and when the field is reversed the hard material remains fixed while an exchange spiral is formed in the soft material [38, 93]. In another manifestation, metallic Gd/Fe systems displaying twisted phases rely on antiferromagnetic coupling between spins at the interface [88, 94-98]. With the SmN/GdN system, however, the interlayer Sm-Gd exchange is ferromagnetic, and the usual hard/soft contrast is of no interest; indeed the fixed layer $(\mathrm{GdN})$ has a coercive field three orders of magnitude smaller than SmN. It is the much stronger Zeeman interaction in GdN than in $\mathrm{SmN}$ that effectively locks the GdN magnetization. Furthermore, the spin-dominant, metallic systems lack the novel combination of electronic and magnetic properties of SmN and GdN, which allow the facility of controlling the concentration and sign of charge carriers without disturbing the ferromagnetic ordered state, and band structure results also show electron and hole channels of majority spin [14].

In our investigation of the interface exchange coupling in $\mathrm{GdN} / \mathrm{SmN}$ multilayers we have used the element selectivity of XMCD at the $\operatorname{Sm~} \mathrm{L}_{2,3}$ and $\mathrm{M}_{4,5}$ edges. We first demonstrate that the $\mathrm{SmN}$ is ferromagnetically exchange coupled to GdN through investigation of a $\mathrm{SmN} / \mathrm{GdN}$ superlattice. We then 
demonstrate that a twisted, or rotating, magnetization develops in ultrathin SmN films coupled to GdN due to interface pinning in the SmN, short-range interionic rare-earth exchange, and the extremely weak Zeeman coupling of $\mathrm{SmN}$. The observed depth dependence of the magnetization is fully consistent with an analytical model based on these competing interactions.

\subsection{Experimental Details}

The attenuation lengths of hard L-edge and soft M-edge X-rays dictated that quite different structures were used for the two investigations. At the L-edge the full thickness of a superlattice of $12 \times(1.5 \mathrm{~nm} \mathrm{SmN} / 9 \mathrm{~nm} \mathrm{GdN})$ was probed through a $100 \mathrm{~nm}$ passivating AlN cap. For the much more surface sensitive M-edge we investigated two samples. The first was a bilayer of $100 \mathrm{~nm}$ GdN/

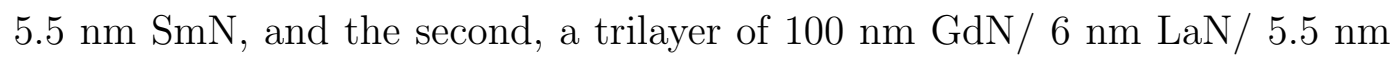
$\mathrm{SmN}$. The non-magnetic LaN layer between the GdN and $\mathrm{SmN}$ was included to block the Gd-Sm exchange interaction in the trilayer. Both of the M-edge samples were passivated with $25 \mathrm{~nm}$ of GaN to prevent sample oxidation.

Samples were grown in a Thermionics ultra-high vacuum system with a base pressure of $1 \times 10^{-8}$ Torr. High purity Gd metal was evaporated at a rate of 0.2 $\AA / \mathrm{s}$ with a $\mathrm{N}_{2}$ partial pressure of $4.5 \times 10^{-4}$ Torr. Sm metal was evaporated at a rate of $0.3 \AA / \mathrm{s}$ under the same $\mathrm{N}_{2}$ pressure. The superlattice was grown on an $\mathrm{MgO}(111)$ substrate, while the bi- and trilayers were grown on c-plane $\mathrm{Al}_{2} \mathrm{O}_{3}$ substrates. All the substrates were outgassed for 1 hour at $700{ }^{\circ} \mathrm{C}$, and heated to $600{ }^{\circ} \mathrm{C}$ during growth. The GaN and AlN capping layers were grown at room temperature with the metal evaporated at a rate of $0.1 \AA / \mathrm{s}$ with an ion source activating the $\mathrm{N}_{2}$. Thicknesses were determined via quartz crystal balances calibrated for SmN, GdN, AlN, and GaN via scanning electron microscope and Rutherford backscattering measurements. The SmN/GdN superlattice was characterized ex situ by XRD, and showed the lattice constant of GdN; as expected the in-plane lattice constant was dominated by the thicker GdN layers in all cases.

Figure 5.2 shows magnetization measurements that were carried out via a Quantum Design SQUID with the field oriented in-plane. Because the much 

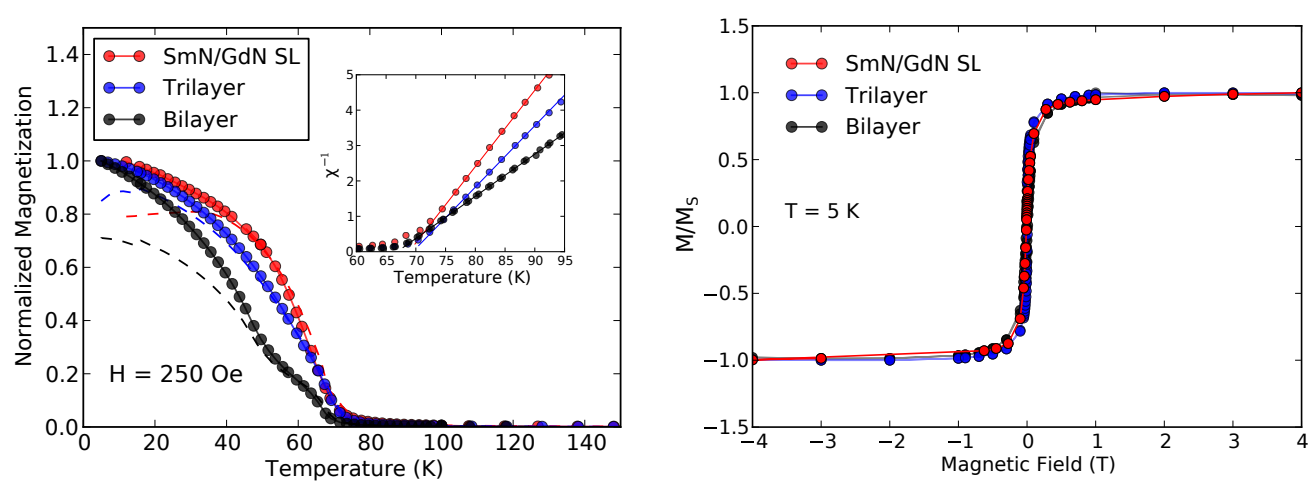

Figure 5.2: SQUID data from bilayer, trilayer, and superlattice all with field in-plane. (a) Temperature dependent magnetization curves (circles denote field-cooled measurements, and dashed lines zero-field cooled) and Curie-Weiss fit to the inverse susceptibility. (b) Hysteresis curves taken at $10 \mathrm{~K}$.

larger magnetic moment of GdN drowns out the signal from SmN, SQUID measurements probe only the GdN magnetization. Curie-Weiss fits to the inverse susceptibility yielded paramagnetic Curie temperatures of $69 \mathrm{~K}, 68 \mathrm{~K}$ and $66 \mathrm{~K}$ for the superlattice, trilayer, and bilayer, respectively. Hysteresis loops measured at $5 \mathrm{~K}$ saturated at $7 \mu_{B}$ per $\mathrm{Gd}^{3+}$ ion. The superlattice and bilayer displayed a coercive field of 120 Oe at $5 \mathrm{~K}$ while the trilayer had a coercive field of $90 \mathrm{Oe}$, all within the range reported for polycrystalline GdN films. [92, 99]

XMCD measurements were performed at temperatures down to $15 \mathrm{~K}$ and fields up to $6 \mathrm{~T}$ at the $\mathrm{Sm}$ and $\mathrm{Gd} \mathrm{L}_{2,3}$ edges on beam line ID12 at the European Synchrotron Radiation Facility (ESRF) in Grenoble. $\mathrm{M}_{4,5}$ edge XMCD was measured at the soft X-ray line ID08 of the ESRF, at temperatures down to $10 \mathrm{~K}$ and in fields up to $4 \mathrm{~T}$. Measurements at the M-edge were necessarily performed only at normal incidence to limit attenuation by a passivating cap. For all of the L-edge XMCD measurements the field and incident beam were directed at $10^{\circ}$ from grazing incidence, in which geometry the very large shape anisotropy ( $4 \pi M \approx 2.5 \mathrm{~T}$, were $M$ is the magnetization) of GdN ensured that the magnetization lay in the plane of the film. At both edges the applied magnetic field was along the X-ray propagation direction. 
The XMCD spectra were obtained by taking the difference of two XAS spectra with the X-ray helicity reversed while the magnetic field was held fixed. This corresponds to the difference between antiparallel and parallel alignments of the helicity and magnetization. XAS spectra have been normalized to the incident photon intensity. XMCD spectra were normalized to the XAS white line intensity at the M-edge and to the edge-jump at the L-edge.

$\mathrm{XMCD}$ at the $\mathrm{Sm} \mathrm{L}_{2}$ edge is the signal of choice for following magnetic hysteresis, for it is stronger than the $\mathrm{L}_{3}$ edge signal. In the superlattice that feature was obscured by magnetic EXAFS (extended X-ray absorption fine structure) from the $\mathrm{Gd} \mathrm{L} \mathrm{L}_{3}$ edge, necessitating the use of the $\mathrm{Sm} \mathrm{L}_{3} \mathrm{XMCD}$ in the superlattice. There was a similar interference in the soft X-ray measurements, where the capping-layer $\mathrm{Ga} \mathrm{L}_{2,3}$ edge introduced a large and variable background in the $\mathrm{Gd} \mathrm{M}_{4,5}$-edge XAS.

Our investigation relies on the use of two common schemes for measuring the X-ray absorption and XMCD spectra, based on the emission of fluorescence (total fluorescence yield, TFY) or electrons (TEY). Below we exploit the differing probing depths of these two schemes in our soft X-ray $\mathrm{M}_{4,5}$ edges, where TFY probes the full $5.5 \mathrm{~nm}$ of the SmN layers while TEY data probe a depth of $\sim 2 \mathrm{~nm}$. Saturation effects distorted the TFY mode at the $\mathrm{Sm} \mathrm{M}_{4,5}$ edges, but nonetheless provide relative comparisons between different samples.

\subsection{L-edge XMCD Results}

We first discuss the hard X-ray results from the $\mathrm{SmN} / \mathrm{GdN}$ superlattice and the homogeneous $\mathrm{SmN}$ sample. Figure 5.3 shows XMCD data from the superlattice at the $\mathrm{Sm} \mathrm{L}_{3}$ edge, compared to the $\mathrm{Sm} \mathrm{L}_{3}$ in homogeneous SmN. These spectra primarily show the dipole transitions from $2 p$ to empty $5 d$ orbitals, with weaker quadrupolar excitations to the $4 f$ shell, and thus signal the strength and sign of the spin and orbital alignments of the $5 d$, and less quantifiably, the $4 f$ shells. The $5 d$ states participate in the ordering through $4 f-5 d$ exchange, though the exchange mechanism between $5 d$ states is not well understood [28]. The XMCD sign reversal shown in Fig. 5.3 between homogeneous $\mathrm{SmN}$ and thin $\mathrm{SmN}$ layers embedded in GdN immediately indicates that Sm-Gd interface 


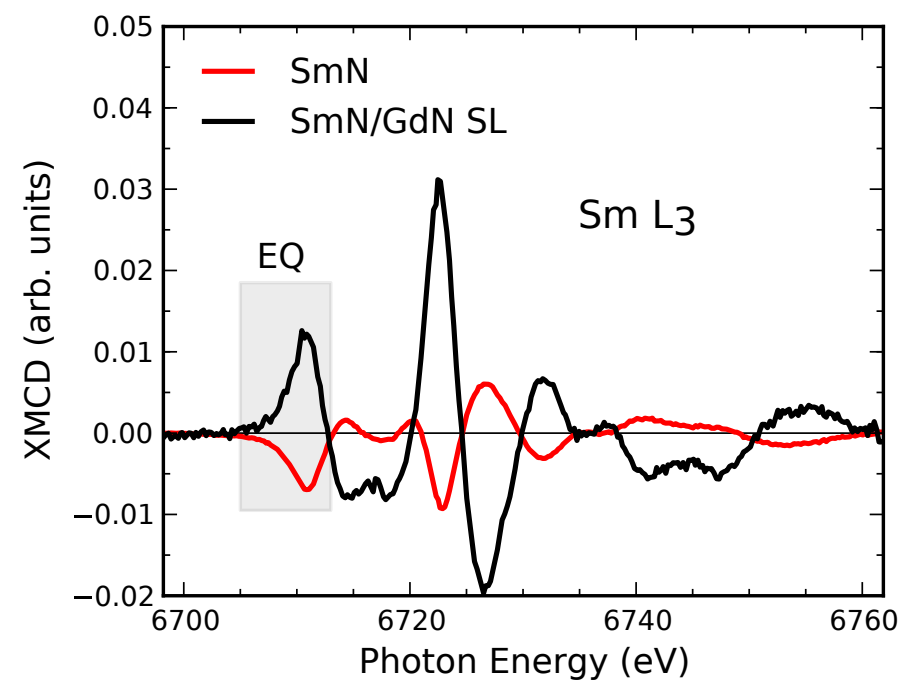

Figure 5.3: XMCD at the $\mathrm{Sm} \mathrm{L}_{3}$ edge in a $\mathrm{SmN} / \mathrm{GdN}$ superlattice (SL) and a homogeneous SmN film taken at $15 \mathrm{~K}$ and a field of $6 \mathrm{~T}$. The signal above $6715 \mathrm{eV}$ is predominantly due to electric dipole (ED) transitions into the $5 d$ shell, and below that the signal is due to electric quadrupole transitions (EQ) into the empty $4 f$ orbitals.

exchange determines the Sm spin alignment, dominating the weak Zeeman interaction that aligns the net, orbital-dominated, moment in homogeneous $\mathrm{SmN}$.

The data displayed in Figure 5.4(a) compares the hysteresis between homogeneous $\mathrm{SmN}$ and $\mathrm{SmN}$ in the superlattice, further demonstrating that the $\mathrm{SmN}$ coercive field in the superlattice is reduced to $\sim 0.01 \mathrm{~T}$, emphasizing that the $\mathrm{SmN}$ magnetization is firmly coupled to the GdN by the exchange interaction across the GdN/SmN interfaces. The inset shows the $\sim 0.01 \mathrm{~T}$ coercive field measured at the $\mathrm{Gd} \mathrm{L}_{2}$ edge. There is an intrinsic sign difference between the most prominent XMCD features at the $\mathrm{Sm} \mathrm{L}_{2}$ and $\mathrm{L}_{3}$ edges, so we have scaled the $\mathrm{L}_{3}$ derived hysteresis by -1 in Figure 5.4(a) in order to indicate the antiparallel spin/orbit alignment between samples, which is clear from the direct $\mathrm{L}_{3}$ edge comparison in Figure 5.3. Examples of the XMCD spectra used to produce Figure 5.4 (a) are shown in Figure 5.4 (b)-(d), along with the XAS spectra.

We now turn to the temperature dependence of the $\mathrm{GdN} \mathrm{L} \mathrm{L}_{3}$ and $\mathrm{SmN} \mathrm{L}_{3}$ 
(a)

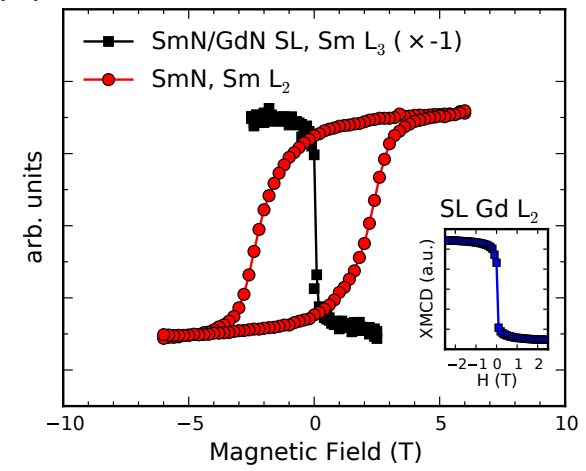

(c)

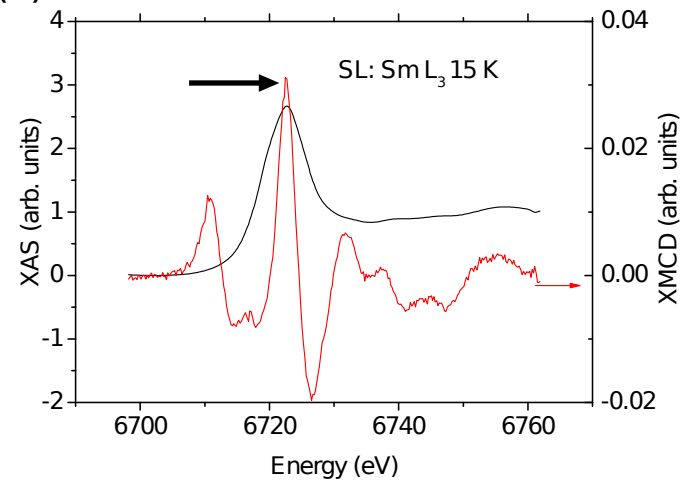

(b)

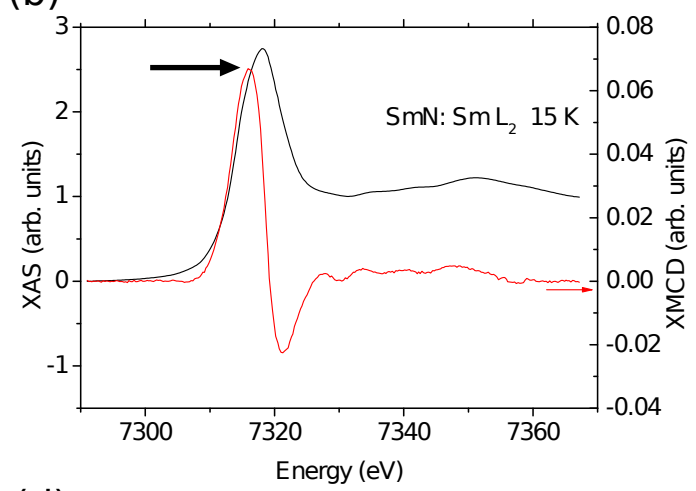

(d)

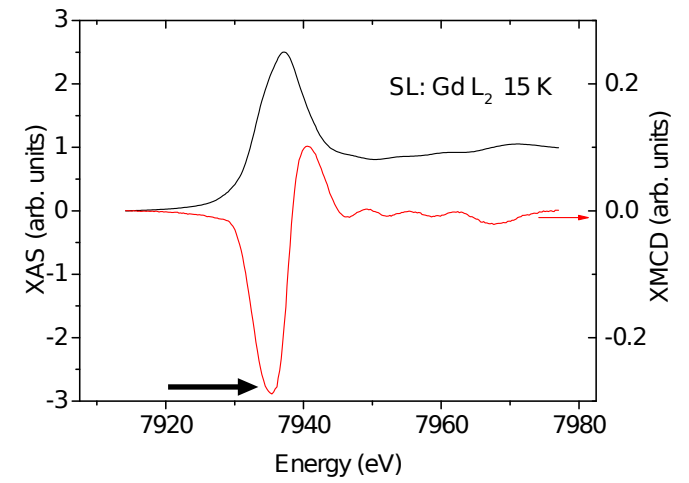

Figure 5.4: (a) XMCD-derived hysteresis taken at $15 \mathrm{~K}$ and $6 \mathrm{~T}$ and measured at the $\mathrm{Sm} \mathrm{L}_{2}$ edge for the superlattice (squares) and at the $\mathrm{Sm} \mathrm{L}_{3}$-edge for the bulk SmN film (circles). The superlattice spectrum was scaled by -1 . The inset shows the $\mathrm{Gd} \mathrm{L} \mathrm{L}_{2}$ edge hysteresis taken at $15 \mathrm{~K}$. Typical XMCD spectra from which the hysteresis curves shown in (a) are derived are shown in (b)-(d) along with the XAS spectra measured at 6 T. The black arrows show the XMCD peaks followed to determine the XMCD hysteresis in (a). 

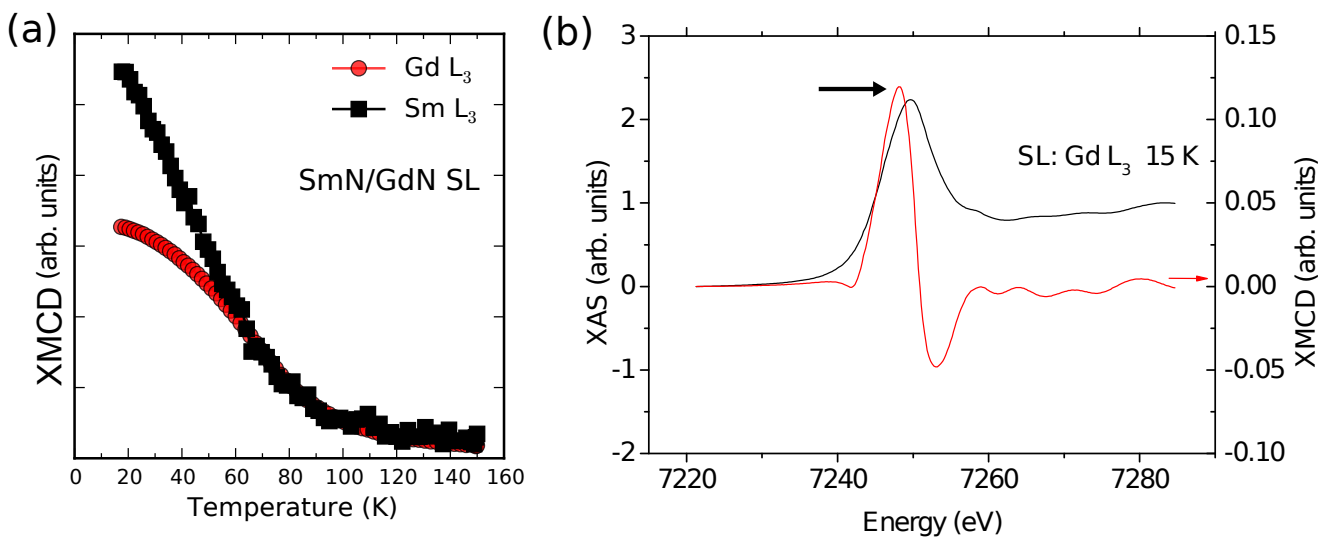

Figure 5.5: (a) Temperature dependence of $\mathrm{Gd} \mathrm{L}_{3}$ and $\mathrm{Sm} \mathrm{L}_{3}$ peaks in a field of $2.5 \mathrm{~T}$ for the $\mathrm{SmN} / \mathrm{GdN}$ superlattice. (b) Superlattice Gd $\mathrm{L}_{3}$-edge XAS and XMCD spectra taken at $15 \mathrm{~K}$ and $6 \mathrm{~T}$.

XMCD, which is shown in Figure 5.5, with an applied field of $2.5 \mathrm{~T}$. The $\mathrm{Sm}$ moments in interface-adjacent ions are again aligned to the GdN moments across the interface at temperatures above the $27 \mathrm{~K}$ Curie temperature of SmN. However, at lower temperatures the Sm moment continues to rise faster than does the rapidly saturating GdN, as the Sm ions deeper in the SmN layer align by the Sm-Sm exchange interaction. At first approximation the $\mathrm{SmN}$ alignment follows the GdN at the interface, with Sm deeper in the SmN layers aligned only well below $27 \mathrm{~K}$. Those inner ions would then not align fully until below the $17 \mathrm{~K}$ minimum temperature in the data. The strong interface coupling between GdN and SmN spin-moments prevails over the orbital-dominated Zeeman coupling in the $\mathrm{SmN}$ layers.

We may also use the results of Chapter 4 to derive more quantitative conclusions about the interface coupling. In Chapter 4 we saw that the experimentally observed SmN magnetic moment of $0.035 \mu_{B}$ per Sm ion could be reproduced within the point charge model with $Z=2.4$ when excited $J$-multiplets are included. The exchange parameter was estimated from the paramagnetic Curie temperature of $27 \mathrm{~K}$. In the superlattice interface exchange clearly modifies this parameter.

If we assume that the effective $\mathrm{SmN}$ exchange is proportional to the $5 d$ spin polarization, then the electric dipole signal at the $\mathrm{Sm} \mathrm{L}_{3}$ edge is a measure of 
Chapter 5 Twisted Magnetization in $S m N / G d N$

\begin{tabular}{lccc}
\hline Exchange parameter & $\left\langle S_{z}\right\rangle$ & $\left\langle L_{z}\right\rangle$ & $\left\langle L_{z}+2 S_{z}\right\rangle$ \\
\hline \hline$J_{e x}=7.9 \mathrm{~K}$ & -0.982 & 1.996 & 0.033 \\
$J_{e x}^{S L}=3 J_{e x}$ & -1.48 & 3.11 & 0.16 \\
\hline
\end{tabular}

Table 5.1: Expectation values of $\mathrm{Sm}^{3+}$ in a cubic crystal field with $Z=2.4$ (See Chapter 4 ). The exchange constant is estimated to be three times larger in the superlattice, resulting in a $50 \%$ larger value of $\left\langle S_{z}\right\rangle$.

the exchange strength in SmN. In particular, it represents the average value of the exchange strength (and spin polarization) through the film, which varies with distance from the $\mathrm{GdN} / \mathrm{SmN}$ interfaces. In the superlattice the electric dipole signal is a factor of $\approx 3$ larger than in homogeneous $\mathrm{SmN}$, thus $J_{e x}^{S L} \approx$ $3 J_{e x}$ where $J_{e x}$ is $7.9 \mathrm{~K}$, as estimated from the SmN Curie temperature. Using $J_{e x}^{S L}$ in the calculation of the $4 f$ magnetic moments results in a value of $\left\langle S_{z}\right\rangle$ increased by $50 \%$ compared to the homogeneous $\mathrm{SmN}$ (see Table 5.1). This value can be compared to the electric quadrupole (EQ) transitions in Figure 5.3 , which represent the transitions to the $4 f$ states. The EQ XMCD is thus proportional to the $4 f$ spin (or orbital) moment. The amplitude of the EQ signal increases by a factor of $60 \%$ in the superlattice as compared to the homogeneous $\mathrm{SmN}$, in good agreement with the calculation.

These increased amplitudes relate only to the average over the $\mathrm{SmN}$ layer thickness. The thin $1.5 \mathrm{~nm} \mathrm{SmN}$ layers consist of 5 atomic planes of Sm ions, thus $\approx 40 \%$ of the Sm ions are interfacial. If only these GdN-adjacent layers experience interface exchange they require an increase in the $5 d$ polarization by a factor of 7.5 to explain the 3-fold enhancement of the EQ XMCD signal above. This seems plausible given the large spin value of $\mathrm{GdN}$, which has a moment of $7 \mu_{B}$, seven times the homogeneous $\mathrm{SmN}$ spin moment calculated in Chapter 4.

\section{$5.4 \quad$ M-edge XMCD Results}

We access the magnetic alignment of $\mathrm{SmN}$ more directly by turning to the soft X-ray $\mathrm{M}_{4,5}$ edges, which represent $3 d \rightarrow 4 f$ transitions and thus signal the spin and orbital alignment in the $4 f$ shell. Figure 5.6(a) sketches the geometry 
of the M-edge measurements, with the magnetic field and X-rays parallel to the surface normal. Figure 5.6(b) shows the XAS spectra for the bilayer and trilayer sample in both TEY and TFY modes. The spectra show the features associated with the multiplet structure of the $\mathrm{Sm}^{3+}$ as shown in Chapter 3 .

Figure 5.6(c) shows the $\mathrm{Sm} \mathrm{M}_{4,5}$-edges XMCD in both TEY and TFY modes for the two samples. For the trilayer, the TFY and TEY spectra in the $\mathrm{SmN}$ layer are in excellent agreement, establishing that $\mathrm{SmN} 4 f$ alignment in the near-surface region and the bulk average are of the same sign, suggesting the $\mathrm{SmN}$ is effectively decoupled from the GdN. In contrast, both TFY and TEY signals are substantially weaker in the bilayer, and the TFY signal is even inverted. The latter is a signature of $\mathrm{SmN}$ that is strongly coupled to the GdN by exchange across the GdN/SmN interface.

To quantify the differences in XMCD between samples we curve fit the spectra to each other over both $\mathrm{M}_{4}$ and $\mathrm{M}_{5}$ edges, instead of simply comparing the intensity at the maximum XMCD signal at $\sim 1077 \mathrm{eV}$. This is to reduce the effects of noise, especially for the bilayer sample. This fitting is reasonable because the strong spin-orbit coupling of the $4 f$ electrons keeps spin and orbital moments firmly aligned relative to each other [65, 100], and so the XMCD sum rules $[55,56]$ imply that the XMCD spectral shape should remain the same between the samples, though with an overall scaling factor. This scaling factor is a measure of how the depth averaged XMCD is different between samples, and thus how the spin/orbital alignment of the $4 f$ magnetic moment varies through the depth. Figure 5.7 shows XMCD spectra which represent the average of multiple measurements and least-squares fits to them.

Fitting of the spectra yields spin/orbital-alignment ratios of bilayer-totrilayer of $R_{\mathrm{TEY}}=0.20 \pm 0.07$ and $R_{\mathrm{TFY}}=-0.12 \pm 0.02$. For the bilayer then, the alignment in the surface $\sim 2 \mathrm{~nm}$ probed by TEY is Zeeman-dominated (i.e., bulk-like $\mathrm{SmN}$ ) but the average through the film is of opposite sign, as was found also in the very thin $\mathrm{SmN}$ layers in the superlattice of the $\mathrm{L}_{2,3}{ }^{-}$ edge study above. We interpret this inhomogeneous alignment in the bilayer as a rotation of the spin and orbital moments through the depth of the film, similar to a domain wall. The distinction is that this rotating or twisted state originates from the interface exchange-Zeeman competition. 
(a)

a)
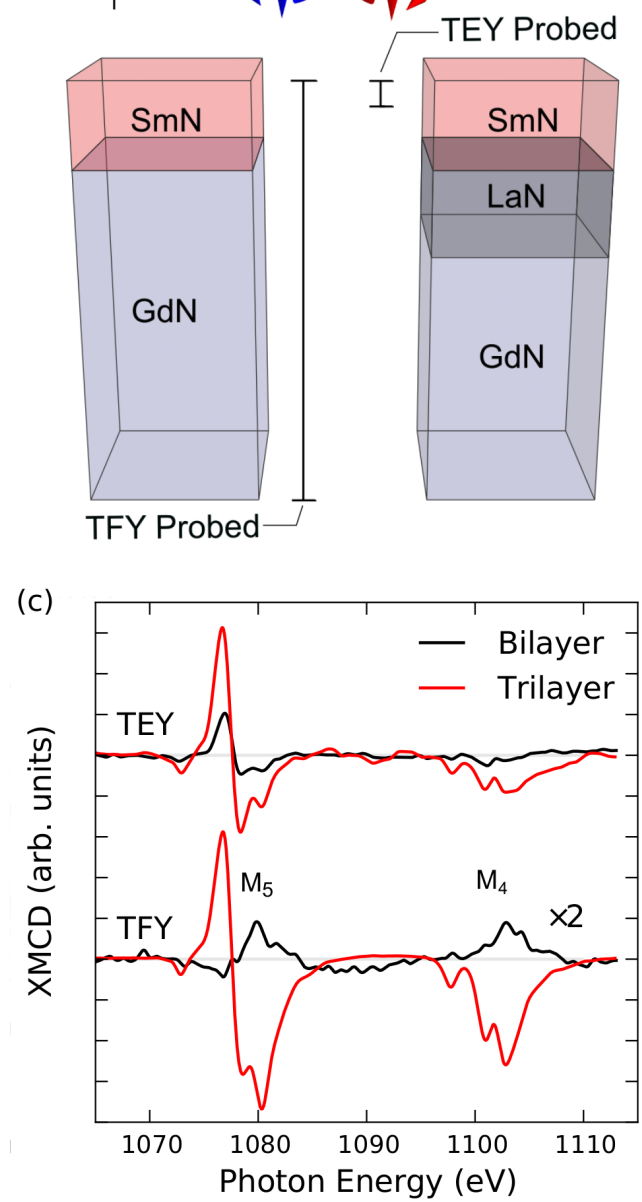

(b)
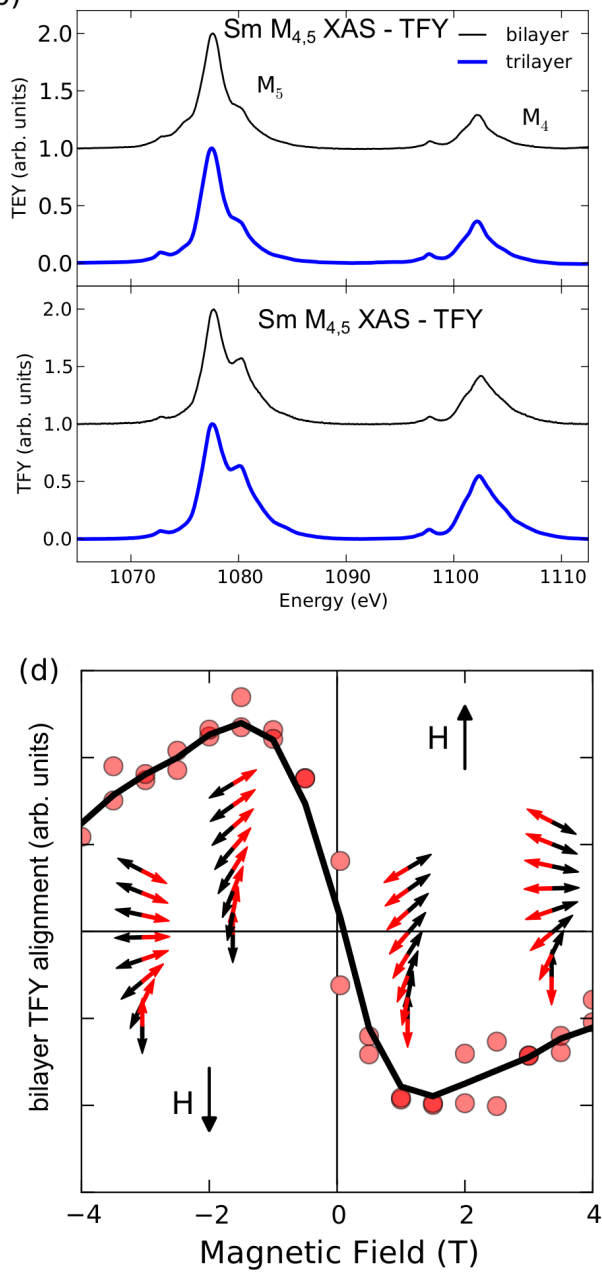

Figure 5.6: (a) Sketch of sample geometry, with magnetic field and X-rays along the surface normal. (b) XAS spectra taken at the $\mathrm{Sm} \mathrm{M}_{4,5}$ edges for both the bilayer and trilayer in TEY and TFY modes. The spectra reveal the expected $\mathrm{Sm}^{3+}$ character present in SmN. (c) XMCD for bilayer and trilayer in TEY and TFY modes, taken with a field of $4 \mathrm{~T}$ and $10 \mathrm{~K}$. (d) Hysteresis derived from a fit of the bilayer TFY spectra. Black line represents a smooth average as a guide to the eye, inset arrows show spin and orbital rotations. 


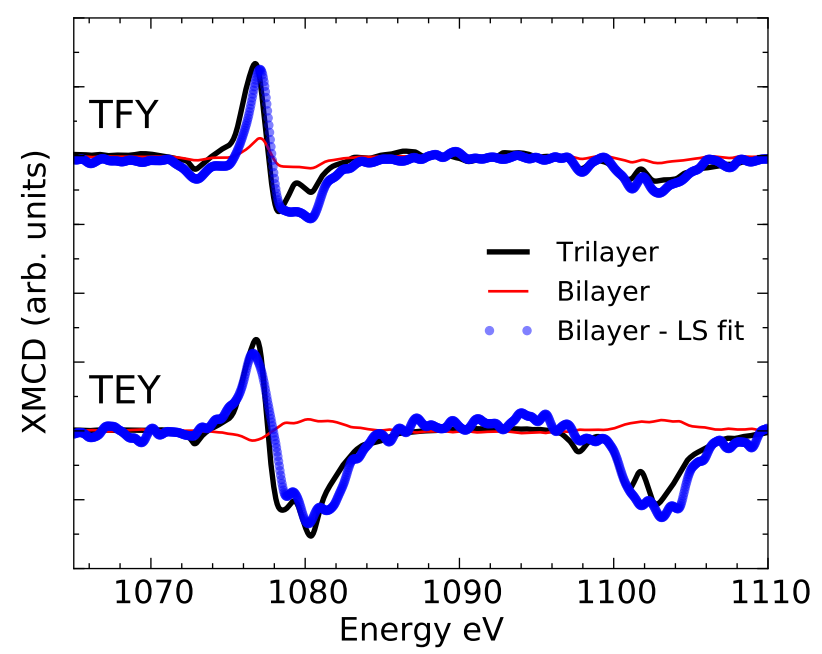

Figure 5.7: Averaged XMCD spectra at the $\mathrm{Sm} \mathrm{M}_{4,5}$ edges. The bilayer spectra have been fit to the trilayer in TEY and TFY modes. The bilayer least-squares (LS) fit (blue circles) represents the bilayer spectra scaled by the fit factor $R_{\mathrm{TXY}}$ for TEY and TFY modes.

Figure 5.6(d) shows an unusual hysteresis curve extracted from the bulk sensitive TFY measurement of the bilayer, where $\mathrm{SmN}$ is deposited directly on GdN. The same fitting procedure mentioned above was used to extract the hysteresis. The Sm $4 f$ alignment in this case shows the same sign inversion seen in the $\mathrm{L}_{2,3}$-edge data in Figures 5.3 and 5.4, but with diminishing alignment with increasing fields larger than $\approx 1.5 \mathrm{~T}$. It is important to note that in the field-normal configuration, the shape anisotropy of GdN prevents a saturated magnetization in applied fields smaller than $\approx 2.5 \mathrm{~T}$. Its magnetization rises approximately linearly with weaker applied fields, but for larger fields the GdN is saturated; between 2 and $4 \mathrm{~T}$ the $4 f$ spins are fully aligned and exert the full Gd-Sm exchange on the $\mathrm{SmN} 4 f$ spin-moment at the interface. In this region the increasing field has the effect of modifying the exchange-Zeeman competition which in turn reduces the bulk averaged XMCD signal as the $4 f$ spin and orbital moments rotate through the film. In the following section we pursue deeper insight into the nature of the twisting, or rotating magnetization. 


\subsection{Analysis and Discussion}

In this section we relate the measured TEY and TFY XMCD results in the bilayer to a model of the twisting SmN magnetization. We consider a one dimensional model of the $\mathrm{SmN}$ magnetization in the bilayer, in which the resulting magnetization profile is determined by the balance among (i) the Sm-Sm exchange energy acting on Sm spin moments, (ii) the Zeeman energy acting on the SmN net moment, and (iii) the demagnetization field of SmN. Our description of the magnetization follows that of Goto et al. [38] and Thiaville \& Fert [81] (briefly discussed in Chapter 2), which treats atomic planes parallel to the $\mathrm{SmN}$ surface as each having a uniform magnetization, such that the problem is reduced to a one dimensional model where each atomic plane is treated as a single effective magnetic moment. In the continuum limit, the magnetic free energy per unit area (at $T=0)$, integrated along the depth $L$ of the $\mathrm{SmN}$ film can then be written as

$$
\mathcal{E}=\int_{0}^{L} \mathrm{~d} z\left[A\left(\frac{\mathrm{d} \theta(z)}{\mathrm{d} z}\right)^{2}-\mathbf{M}_{s} \cdot \mathbf{H}-\frac{1}{2} \mathbf{M}_{s} \cdot \mathbf{H}_{d}+E_{\text {anis }}\right]
$$

where $A$ is the exchange stiffness, $\mathbf{M}_{s}$ is the saturation magnetization of the $\mathrm{SmN}, \mathbf{H}=H \hat{\mathbf{z}}$ is the applied field, and $L=5.5 \mathrm{~nm}$ is the thickness of the SmN film. $\theta(z)$ is the depth-varying angle between $\mathbf{H}$ and the spin-moment $\mathbf{m}_{S}$ (see Figure 5.8(a)) We note that for the thin film geometry with the field perpendicular to the plane the demagnetization field is given by $\mathbf{H}_{d}=$ $-4 \pi \mathbf{M}_{s}=-0.01 \mathrm{~T}$ which can be neglected in comparison with the Zeeman energy in the large $(4 \mathrm{~T})$ fields we consider here. The $E_{\text {anis }}$ term corresponds to magnetocrystalline anisotropy, however there are no studies of its effects in $\mathrm{SmN}$ [30] and our results suggest it is only a weak correction. Out of plane rotation (i.e. the plane formed by $\mathbf{H}$ and the magnetization) can be ignored as this creates a static field which tends to only increase the energy.

Simplifying Equation (5.1) we arrive at

$$
\mathcal{E}=\int_{0}^{L} \mathrm{~d} z\left[A\left(\frac{d \theta(z)}{d z}\right)^{2}-\mathbf{M}_{s} \cdot \mathbf{H}\right]
$$


This equation is formally identical to that used in exchange spring-systems when anisotropy is negligible (Chapter 2) [38, 93]. The crucial distinction is that the Zeeman term adopts the opposite sign as found in conventional spindominant systems, due to the fact that the net moment is antiparallel to the $\mathbf{m}_{S}$, hence $-\mathbf{M}_{s} \cdot \mathbf{H}=M_{s} H \cos \theta(z)$. The Zeeman energy is thus minimized when $\theta(z)=\pi$. The exchange stiffness $A=4 J_{e x} S^{2} / n a^{*}$ involves the exchange constant $J_{\text {ex }}$ estimated from the experimental Curie temperature of $\mathrm{SmN}$ and the number $n$ of nearest $\mathrm{Sm}$ ion neighbors. The nearest neighbor $\mathrm{Sm}^{3+}$ distance is $a^{*}$, related to the $\mathrm{SmN}$ lattice constant by $a^{*}=a / \sqrt{2}$.

Equation (5.2) can be minimized analytically (see Appendix B for details) to yield the most energetically favorable configuration, as carried out by Goto et al. [38] for an exchange-spring system, yielding the following expression for $\theta(z)$ :

$$
\theta(z)=2 \arcsin \left[K^{-1}(\alpha L) \operatorname{sn}(\alpha z)\right],
$$

where $\alpha=\sqrt{H M_{S} / 2 A}$, and the functions $K^{-1}$ and sn are the inverse of the complete elliptic function of the first kind and the Jacobi elliptic function of the first kind, respectively. The boundary conditions were chosen such that $\theta(L)=0$ (Sm spin is aligned with the Gd spin at the SmN-GdN interface) and $d \theta(z) /\left.d z\right|_{z=0}=0$ (SmN free surface). These boundary conditions account for the magnetically soft GdN being rigidly fixed parallel to the applied field due to its large Zeeman interaction. This fixed GdN then acts as the rigid pinning layer for the $\mathrm{SmN}$ at the interface.

A twisted phase develops on a scale of $\ell=\pi / 2 \sqrt{2 A / H M_{S}}$; below this thickness a uniform magnetization $(\theta(z) \equiv 0)$ is the only solution to Equation (5.3) In an applied field of $4 \mathrm{~T}$ this corresponds to $\ell \approx 4 \mathrm{~nm}$, on the order of the SmN film thickness. Figure 5.8(b) shows the calculation of the net moment and spin-moment projected on the $z$-axis (i.e., $m \cos \theta(z)$ and $m_{S} \cos \theta(z)$ ), as a function of the depth $z$ in the $4 \mathrm{~T}$ field. The scale of the twist increases in a field of $2 \mathrm{~T}$ as the Zeeman energy weakens, illustrated in Fig. 5.8(a). In this plot we have assumed that the spin moment $m_{S}$ is the fully polarized value of $2.5 \mu_{B}$, which according to the calculation in Chapter 4 is reduced to around $1 \mu_{B}$ per Sm ion. However, in the following we show that the value of $m_{S}$ does 


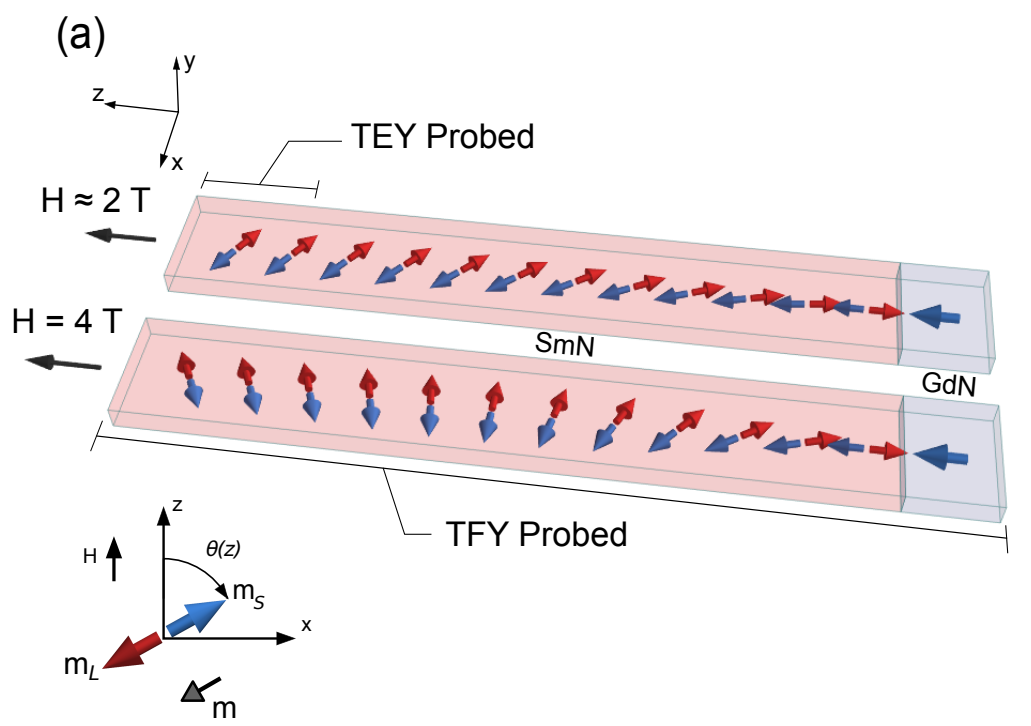

(b)

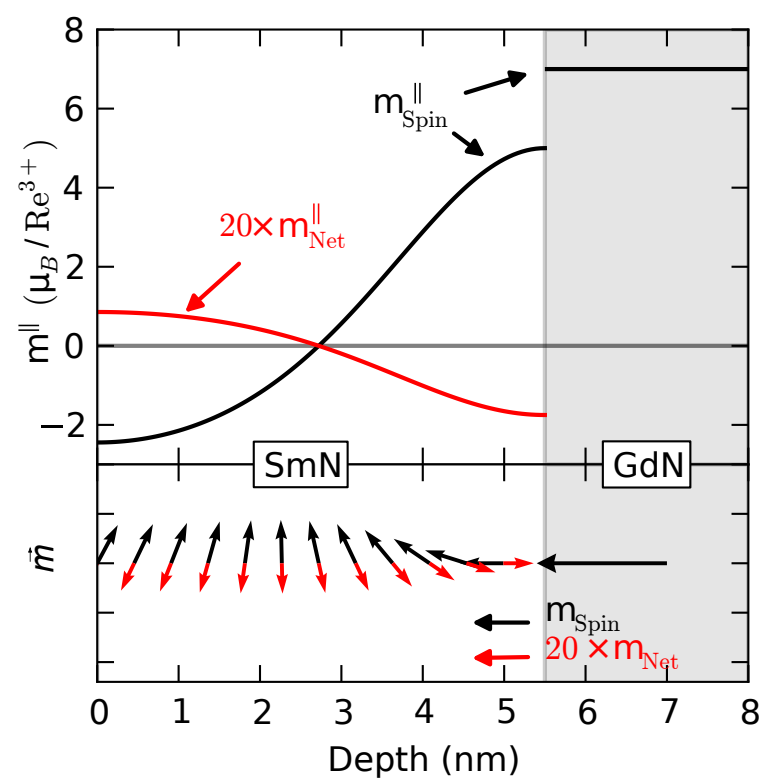

Figure 5.8: (a) A sketch of the in-plane twisted magnetization structure near the SmN-GdN interface with spin moments (blue) and orbital moments (red). (b) Calculation of the spin and net moment as a function of thickness in the $\mathrm{SmN}$ layer of the bilayer. The Gd moment is fixed at $7 \mu_{B}$ through the GdN film, and the Sm spin-moment is pinned at the $\mathrm{SmN} / \mathrm{GdN}$ interface to its maximum value of $5 \mu_{B}$. 


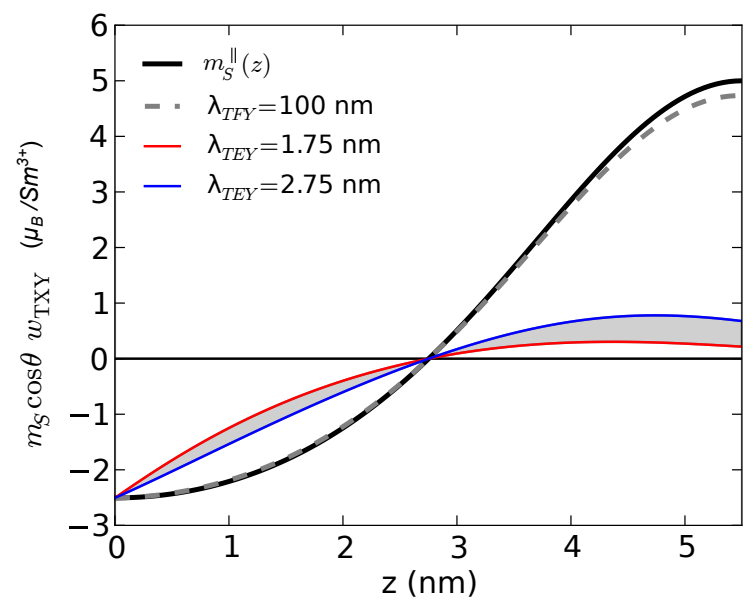

Figure 5.9: A plot of the $m_{S} \cos \theta(z) \exp \left(-z / \lambda_{\mathrm{TXY}}\right)$ for various values of $\lambda_{\mathrm{TXY}}$. For example, for the TEY ratio, we calculate $R_{\mathrm{TEY}}=0.24$ for $\lambda_{\mathrm{TEY}}=$ $1.75 \mathrm{~nm}$ and $R_{\mathrm{TEY}}=0.19$ for $\lambda_{\mathrm{TEY}}=2.25 \mathrm{~nm}$. The experimental value of $R_{\mathrm{TEY}}^{\exp }=0.20$ thus corresponds to the value of $\lambda_{\mathrm{TEY}} \approx 2.15 \mathrm{~nm}$.

not affect the comparison with experiment.

The resulting depth profile of the net $\mathrm{SmN}$ moment projected along the zaxis, $m \cos \theta(z)$, can be compared to the XMCD spectra by accounting for the depth-averaging of the XMCD measurement, in combination with the effective sampling depth in the TXY (TEY or TFY) measurement schemes, $\lambda_{\mathrm{TXY}}$. The finite sampling depth $\lambda_{\mathrm{TXY}}$ in the TXY mode results in a detection efficiency $w_{\mathrm{TXY}}=e^{-z / \lambda_{\mathrm{TXY}}}$ from a depth $z[41]$. Thus we can approximate the depth averaged XMCD measurement as returning an effective net moment of

$$
\langle m\rangle_{\mathrm{TXY}}=\frac{1}{L} \int_{0}^{L} \mathrm{~d} z m \cos \theta(z) e^{-z / \lambda_{\mathrm{TXY}}}
$$

Absolute values of $m_{S}$ and the orbital moment $m_{L}$ can in principle be extracted by applying the XMCD sum rules, however they require much greater signal-to-noise ratios than available with the present data. Instead we note that $m_{S}$ and $m_{L}$ in both samples are fixed antiparallel by the strong spin-orbit coupling, and the XMCD spectra remain proportional. The ratios of $\langle m\rangle_{\mathrm{TXY}}$ between the bilayer and trilayer are thus expressions for the experimentally 
determined ratios, which simply reflects the depth-average of $\cos \theta(z)$ within the bilayer. The calculated ratio $R_{\mathrm{TXY}}=\langle m\rangle_{\mathrm{TXY}}^{\text {bilayer }} /\langle m\rangle_{\mathrm{TXY}}^{\text {trilayer }}$ in the TFY mode gives $R_{\mathrm{TFY}}=-0.11$, in excellent agreement with the measured ratio of $-0.12 \pm$ 0.02. $R_{\mathrm{TFY}}$ is insensitive to the precise value of $\lambda_{\mathrm{TXY}}(\approx 100 \mathrm{~nm})$, as $d \ll \lambda_{\mathrm{TFY}}$; the fluorescence is effectively unattenuated. The TEY-channel ratio depends more strongly on $\lambda_{\mathrm{TEY}}$; the experimental value of $R_{\mathrm{TEY}}=0.20$ is returned for an electron escape depth of $\lambda_{\mathrm{TEY}}=2.15 \mathrm{~nm}$, which is consistent with the expected range $[41,45]$. The resulting spin-moment depth profiles calculated using $J_{\mathrm{ex}}=7.9 \mathrm{~K}$ are shown in Fig. 5.9, where $m_{S} \cos \theta(z) \exp \left(-z / \lambda_{T X Y}\right)$ is plotted as a function of depth for the various $\lambda_{\text {TXY values, along with the actual }}$ spin moment profile, $m_{S}(z) \cos \theta(z)$. The excellent agreement of this analytical model with the experimentally measured spectra thus strongly supports the mechanism of exchange-Zeeman competition driving the twisted magnetization in the $\mathrm{SmN}$ layer coupled to $\mathrm{GdN}$.

The effect of the enhanced interface exchange observed in the SmN/GdN superlattice has been ignored in this analysis, as is not expected to qualitatively alter the main results. The much thicker $\mathrm{SmN}$ in the bilayer compared to the superlattice contains approximately 15 atomic planes of $\mathrm{Sm}$ ions, and means that the enhanced exchange at the interface layer would have only a small effect. The agreement achieved by using only experimental parameters and reasonable values of $\lambda_{\mathrm{TXY}}$ is encouraging and indicates that other effects, including bulk and surface anisotropies are only weak corrections to the exchange and Zeeman dominated contributions. We add that the continuum approximation leading to Eq. (5.2) has been shown to be in good agreement with more exact treatment using a discretized version of the model, even down to a few monolayers [101].

\subsection{Conclusion and Outlook}

In summary, we have observed a novel twisted magnetization phase in a $\mathrm{SmN} / \mathrm{GdN}$ bilayer by exploiting the depth dependence of the electron-yield and fluorescence-yield detection modes at the rare-earth M-edge XMCD. Remarkably, it is in fact the hard magnetic SmN layer which develops the twisted 
phase, while the soft GdN film remains fixed parallel to the field. The interfacial pinning of the $\mathrm{SmN}$ moment to GdN was also clearly demonstrated in the L-edge XMCD measurements, showing that the ferromagnetic GdN-SmN exchange coupling is responsible for the pinning. The crystal field calculations of Chapter 4 provided an explanation of how the interface-exchange altered the $\mathrm{SmN}$ if spin and orbital moment alignments, in good agreement with experiment.

The relatively simple model used to describe the $\mathrm{SmN}$ magnetization is just a starting point for exploring these twisted phases. Acquiring XMCD data at the M-edge with higher signal-to-noise ratios would allow for the application of more sophisticated modelling, however experiments are difficult to perform on this system for a few reasons. One is the extremely small moment of $\mathrm{SmN}$ which leads to intrinsically weak dichroism, while the necessity for a capping layer leads to a low signal-to-noise ratio. The in-situ sample growth possible at a few XMCD beamlines in synchrotrons around the world would remove the need for a capping layer during measurements, allowing for much higher signal-to-noise ratios to be obtained.

The decoupling of the $\mathrm{SmN}$ and GdN magnetization in the $\mathrm{SmN} / \mathrm{LaN} / \mathrm{GdN}$ structure points towards magnetic tunnel junctions, especially attractive within the RENs owing to their epitaxial compatibility across the series. The appearance of a twisted phase in the $\mathrm{SmN} / \mathrm{GdN}$ system also holds intriguing possibilities for spintronic applications, owing to the semiconducting nature of the pair coupled with the orbital-dominant magnetism of SmN. For example, the tuning of the twisted phase length scale $\ell \sim \sqrt{A / H M_{s}}$ for given fields can be achieved through doping, or replacement, with other rare-earth elements, thus modifying the exchange $A$ and the saturation magnetization $M_{s}$ [62]. The ability to control the scale of what is effectively a domain-wall width in intrinsic ferromagnetic semiconductor heterostructures also allows for the opportunity to explore spin-orbit torques in a novel system. 
Chapter 5 Twisted Magnetization in SmN/GdN 


\section{Chapter 6}

\section{Twisted Magnetization in a NdN/GdN Superlattice}

\subsection{Introduction}

In this chapter, we pursue the study of $\mathrm{NdN} / \mathrm{GdN}$ superlattices, a system which shares some similarities to the $\mathrm{SmN} / \mathrm{GdN}$ system investigated in Chapter 5 . $\mathrm{NdN}$, like $\mathrm{SmN}$, is an orbital-dominant ferromagnet, making it a good candidate to explore twisted phases relying on exchange-Zeeman competition. The $\mathrm{Nd}^{3+}$ ion is an $f^{3}$ system, and Hund's rules yield an $L=6$ orbital and $S=3 / 2$ spin contribution to the total angular momentum, which has the ground state value $J=|L-S|=9 / 2$. In contrast to the near-zero ferromagnetic moment of $\mathrm{SmN}, \mathrm{NdN}$ yields a much larger orbital-dominant moment between 0.9 $2.1 \mu_{B}$ per ion in the ferromagnetic phase, as we saw in Chapter 4. The net NdN moment has a magnitude of 15 - $30 \%$ of the GdN moment.

With this sizable NdN moment we can expect that the GdN is influenced by exchange at the interface with $\mathrm{NdN}$, and we may expect that twisted magnetizations will form in both $\mathrm{NdN}$ and GdN layers. This is in contrast to the case of the $\mathrm{SmN} / \mathrm{GdN}$ interface, where the small Zeeman coupling of the $\mathrm{SmN}$ meant that the GdN was aligned solely by its Zeeman interaction, which kept it rigidly aligned parallel to the field.

This large NdN moment has another important effect. While the GdN signal dominates the overall magnetization of a NdN/GdN superlattice, the 
$\mathrm{NdN}$ contribution is still observable, in contrast to the $\mathrm{SmN} / \mathrm{GdN}$ system. This means that it is possible to see clear signals of the NdN in standard lab-based magnetometry techniques. As we shall see, magnetometry, coupled with the element-specific probe of XMCD provides us a detailed picture of a remarkable system in which a twisted magnetization forms due to the presence of a large orbital-dominant moment. This investigation also serves as a confirmation for the mechanism of the orbital-driven twisted phase observed in the $\mathrm{SmN} / \mathrm{GdN}$ systems.

In this chapter we focus on a single $\mathrm{NdN} / \mathrm{GdN}$ superlattice, outlining the sample preparation and characterization, and then continue to a study of the magnetic properties. First we discuss results from magnetometry, which investigates the temperature and field dependence of the sample. Next we turn to an XMCD study of the superlattice, measured at the $\mathrm{Nd} \mathrm{L}_{2,3}$ edges, which probes the $\mathrm{Nd} 5 d$ states which participate in mediating the exchange. In both magnetization and XMCD studies we present clear evidence of a twisted magnetization phase in the NdN and GdN layers.

\subsection{Experimental Details}

The sample investigated in this chapter is a $10 \times(10 \mathrm{~nm} \mathrm{NdN} / 10 \mathrm{~nm} \mathrm{GdN}) \mathrm{su}-$ perlattice grown on a GaN(0001) buffer layer with a sapphire (0001) substrate. The general procedure was the same as used in Chapter 5 and discussed in Chapter 2. Growth took place in a $\mathrm{N}_{2}$ pressure of $2 \times 10^{-4}$ Torr, NdN and GdN were evaporated with an electron gun at a rate of 0.1 to $0.2 \AA /$ s. The substrate was outgassed for one hour at $600^{\circ} \mathrm{C}$, while the temperature was kept at $390^{\circ} \mathrm{C}$ during the growth. A GaN capping layer of approximately $50 \mathrm{~nm}$ was grown at room temperature at a rate of $0.1 \AA / \mathrm{s}$ with a plasma source exciting the $\mathrm{N}_{2}$ in a pressure of $2 \times 10^{-4}$ Torr. Figure 6.1 shows a $2 \theta$ XRD scan of the superlattice and from a substrate for comparison. The reflection at $31^{\circ}$ corresponds to overlapping GdN and $\mathrm{NdN}$ [111] reflections. Also visible is the $\mathrm{GdN} / \mathrm{NdN}[220]$ reflection at $53^{\circ}$, indicating that the film is polycrystalline.

Magnetization measurements were carried out via a Quantum Design SQUID and vibrating sample magnetometer (VSM). These measurements were all car- 


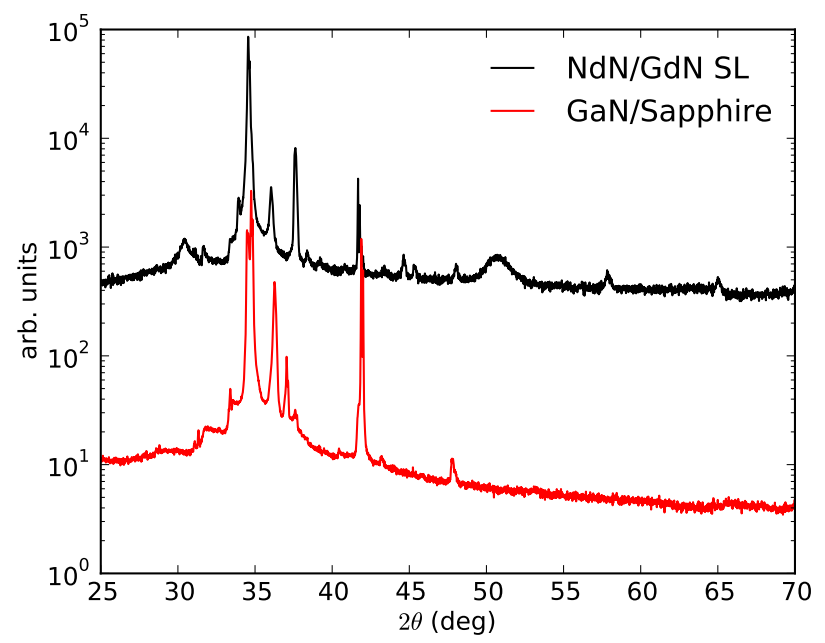

Figure 6.1: XRD pattern from the NdN/GdN superlattice with substrate, a $\mathrm{GaN}(0001)$ buffer layer on $\mathrm{Al}_{2} \mathrm{O}_{3}$ (0001).

ried out with the field in-plane.

\subsection{Magnetization Results}

We begin by examining the "global" magnetization of the superlattice, which is a sum of contributions from the individual GdN and NdN layers. Figure 6.2 displays the zero-field cooled (ZFC) and field cooled (FC) magnetization curves. In the ZFC measurement, the sample is cooled from room temperature to $5 \mathrm{~K}$ in zero field; the magnetization is then measured as the temperature is increased in an applied field of 250 Oe. In the FC process, the magnetization is measured with decreasing temperature in a field of 250 Oe.

We see that in both curves, as the temperature is decreased, the magnetization increases rapidly starting at about $70 \mathrm{~K}$ until approximately $50 \mathrm{~K}$, corresponding to the onset of ferromagnetism in the GdN layers. The region above $\approx 70 \mathrm{~K}$ corresponds to the paramagnetic phases of $\mathrm{GdN}$ and NdN. We use a Curie-Weiss approach to fit the susceptibility, assuming that the GdN 


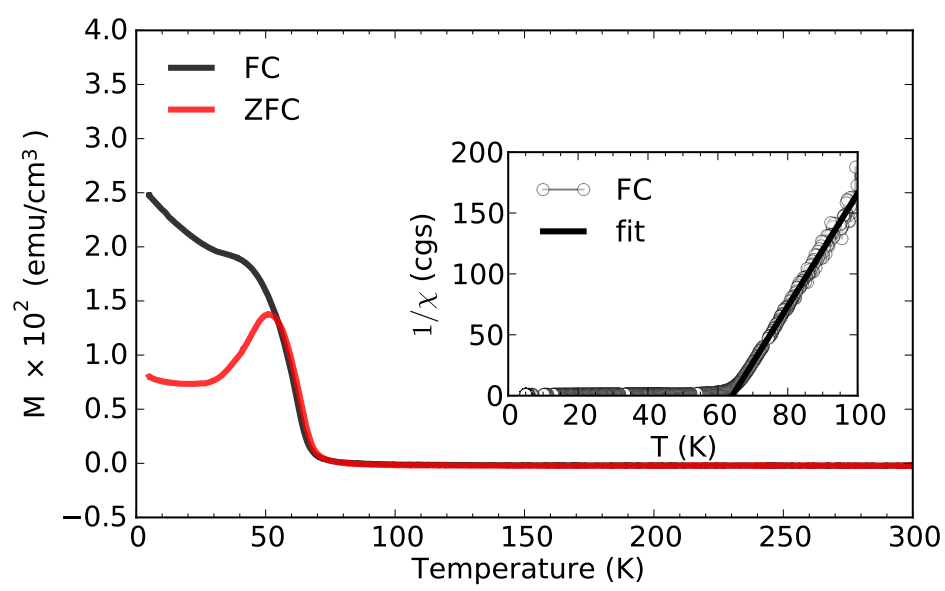

Figure 6.2: Temperature dependent magnetization of the NdN/GdN superlattice in a 250 Oe field, showing both field-cooled (FC) and zero-field cooled (ZFC) measurements. Inset shows Curie-Weiss behavior in the inverse susceptibility taken from the FC measurement.

dominates the paramagnetic response. In this case $\chi$ is given by

$$
\chi=M / H=\frac{A}{T-\theta_{p}}+\chi_{0}
$$

and $\chi_{0}$ is a temperature independent background primarily due to the diamagnetic signal from the substrate. After removing the background signal $\chi_{0}$, we may then plot $\chi^{-1}$, which should be proportional to $T-\theta_{p}$ in the high temperature region above the $T_{C}$ of both $\mathrm{NdN}$ and $\mathrm{GdN}$, shown in the inset of Figure 6.2. The linear behavior is clearly visible, yielding a $\theta_{p}=65 \pm 1 \mathrm{~K}$, expected for polycrystalline $\operatorname{GdN}[12,57,92]$. One might expect a contribution from the NdN layers in this approach, however it would be strongly suppressed due to the smaller moment and lower $T_{C}$. As an estimate, in the Curie-Weiss theory the constant $A$ in Equation 6.1 is proportional to $m_{\text {eff }}^{2}$, where $m_{\mathrm{eff}}=g_{J} \mu_{B} \sqrt{J(J+1)}$ is the effective paramagnetic moment. This value is roughly 5 times larger in $\mathrm{GdN}$, and at $85 \mathrm{~K}$ the factor of $\left(T-\theta_{p}\right)^{-1}$ is about 3 times larger in GdN than for $\mathrm{NdN}$, so the overall $\mathrm{NdN}$ signal at $85 \mathrm{~K}$ should be about 15 times weaker for NdN, explaining the Curie-Weiss behavior with the $\theta_{p}$ of $\operatorname{GdN}$. 
Below the GdN $T_{C}$, the rapidly increasing magnetization continues until around $50 \mathrm{~K}$. In the range of $30-40 \mathrm{~K}$ the NdN should become ferromagnetic, and indeed we see a change in slope in both curves in this temperature range. It is difficult to pinpoint the precise $T_{C}$ of $\mathrm{NdN}$ in the superlattice, however we assume that it corresponds approximately to the values discussed in Chapter 4 , which ranged from $\approx 35$ to $42 \mathrm{~K}$. The ZFC magnetization has different behavior in the low temperature region due to the random orientation of the domains in the ZFC process, which reduces the magnetization compared to the FC measurement. We note that the low temperature behavior of the FC magnetization differs from that in homogeneous GdN samples [10, 12] which approach saturation as a ferromagnetic Brillouin function. This appears to be due to the ferromagnetic NdN influencing the net magnetization of the superlattice. To further elucidate the interplay between ferromagnetic NdN and GdN, we turn to field-dependent magnetization measurements.

Figure 6.3 shows the superlattice $M-H$ loop taken at $5 \mathrm{~K}$. The sample was first zero-field cooled to $5 \mathrm{~K}$, with the magnetization then increased to $6 \mathrm{~T}$. The blue curve represents the subsequent measurement as the field is decreased from $6 \mathrm{~T}$, while the red curve was measured for increasing field. Examining the blue curve, upon decreasing from $6 \mathrm{~T}$ and reaching $H=0$ (see inset), we clearly see that the remanent magnetization $M_{R}=-0.3 \times 10^{2} \mathrm{emu} / \mathrm{cm}^{3}$ is negative. The coercive field, $H_{C}=-0.02 \mathrm{~T}$, is negative as well in this case. The negative sign for $H_{C}$ and $M_{R}$ indicates that the net magnetization of the superlattice switches sign before $H$ does. Furthermore, as the field decreases, there is a change in slope between $-2 \mathrm{~T}$ and $-4 \mathrm{~T}$, which cannot be ascribed to bulk GdN or NdN behavior, which each have coercive fields well below $2 \mathrm{~T}$ in magnitude.

The negative remanent and coercive fields point to complex behavior in the superlattice, and indicate that the hysteresis curve cannot be interpreted within a model of two non-interacting ferromagnets, in which the global magnetization is simply the sum of individual layers having bulk-like magnetization. Exchange bias systems where ferromagnetic films are coupled to antiferromagnetic films may also exhibit complex hysteresis loops [102], however, they are qualitatively different systems, and can be viewed as ferromagnetic systems 

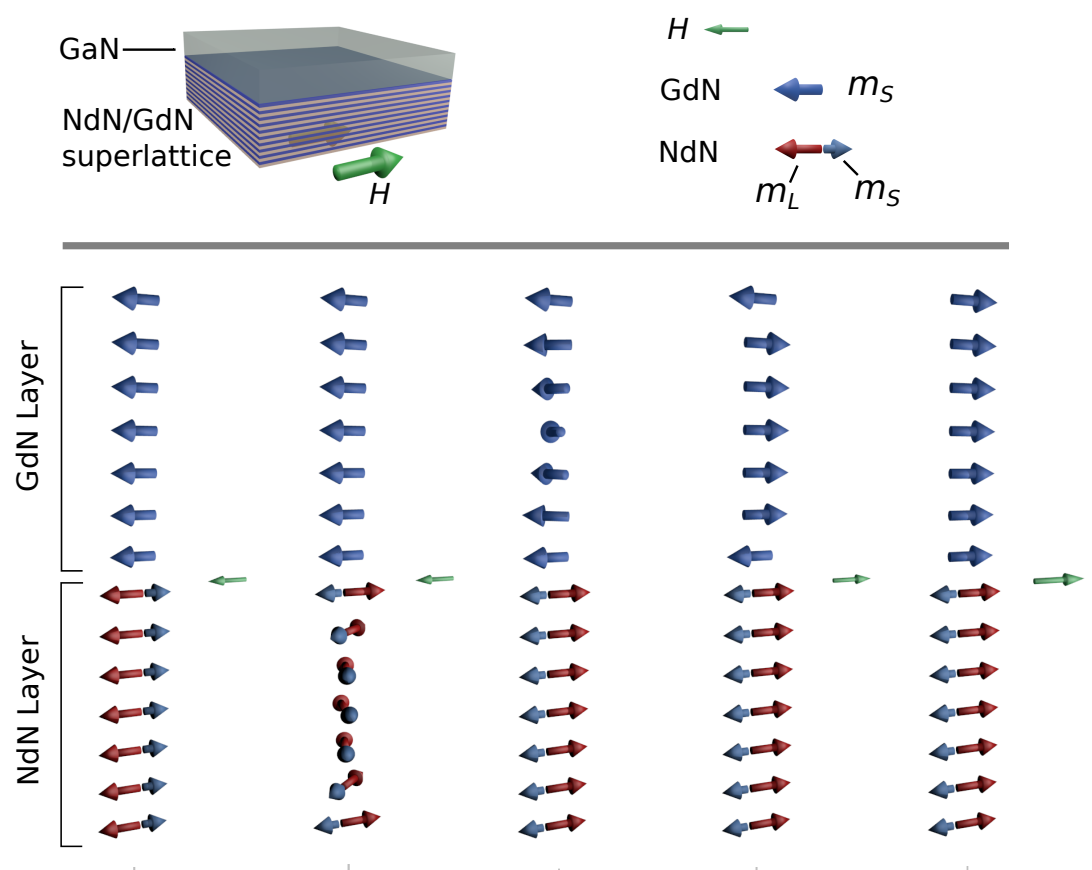

(5)

(4)

(3)

(2)

(1)

Decreasing

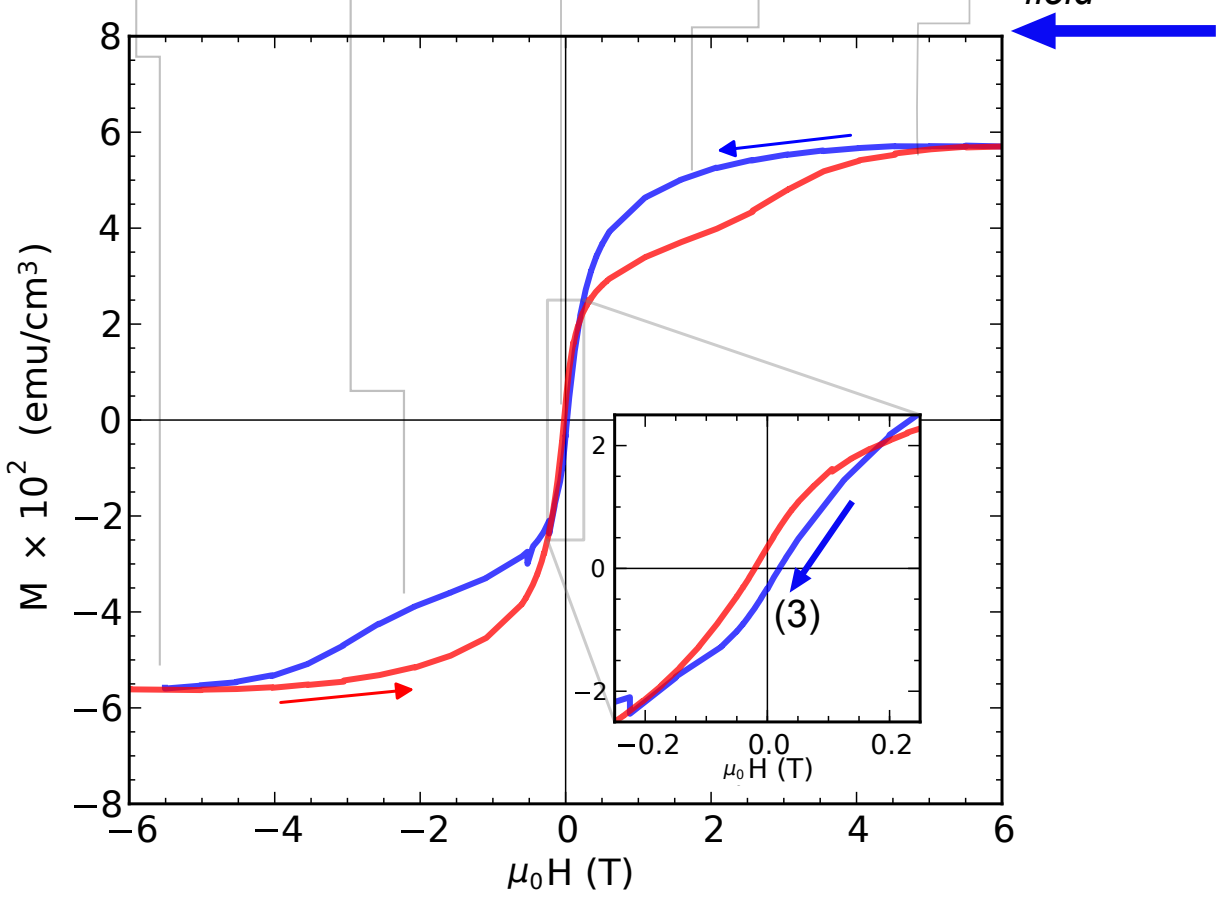

Figure 6.3: $M-H$ plot for the $\mathrm{NdN} / \mathrm{GdN}$ superlattice, at $5 \mathrm{~K}$, with field in-plane, after zero-field cooling. Sketches of the various moment arrangements (1)-(5) in the GdN and NdN layers are given as the field decreases from saturation $6 \mathrm{~T}$; the sketches should thus be read from right to left. 
where the origin of the hysteresis is shifted by some exchange-bias field $H_{E B}$.

How can we explain the negative remanent field $M_{R}$ ? We first of all assume at that $5 \mathrm{~K}$ and $\mu_{0} H=6 \mathrm{~T}$ the moments are close to their saturation values, approximately $7 \mu_{B}$ per ion for GdN and $0.9-2.1 \mu_{B}$ per ion for $\mathrm{NdN}$. In this case the NdN moment is only $16-30 \%$ of the GdN moment. The only reasonable explanation for the negative magnetization at remanence is for the GdN, with its much larger moment, to have its magnetization direction partially reversed when $H=0$. The reversal of the GdN can then be explained if ferromagnetic exchange coupling between NdN and GdN spins is present at the interfaces. Because NdN is an orbital dominant ferromagnet, this FM exchange coupling mimics antiferromagnetic exchange coupling: when the spin moments are aligned the net magnetizations are antiparallel. The NdN, with its large $(\approx 1.5 \mathrm{~T}$ in bulk $\mathrm{NdN})$ coercive field at $5 \mathrm{~K}$, is unlikely to switch due to its large anisotropy energy, and therefore the interface exchange causes the GdN to reverse while the NdN remains fixed, acting as a hard anchoring layer. This situation is sketched in Figure 6.3, where both the NdN and GdN layers have their magnetization sketched in configurations corresponding to points on the blue (decreasing field) part of the $M-H$ curve. In the following we discuss the magnetization configurations in more detail.

Starting in configuration (1) in Figure 6.3, at $6 \mathrm{~T}$, the GdN and NdN layers have their magnetization aligned fully along the field. Upon decreasing the field to configuration (2), the interface-adjacent GdN layers begin to be aligned by ferromagnetic exchange with the NdN, and thus reverse their direction. The $\mathrm{NdN}$ layer is rigid due to its large anisotropy compared to GdN. Upon reaching zero field (3), the NdN maintains its alignment, while the GdN alignment is dominated by interface exchange. The GdN magnetization reversal nucleates from the interfaces and a twisted magnetization is formed such that the net magnetization of the $\mathrm{GdN}$ is negative, and large enough to make the global superlattice magnetization negative. As the field becomes negative in (4), the GdN is now fully aligned with the field, while the innermost NdN layers begin to reverse their magnetization due to the large Zeeman coupling, forming a twisted magnetization. Finally, when the field reaches $-6 \mathrm{~T}$ in configuration (5), the NdN magnetization and GdN are both fully aligned by the field. The 

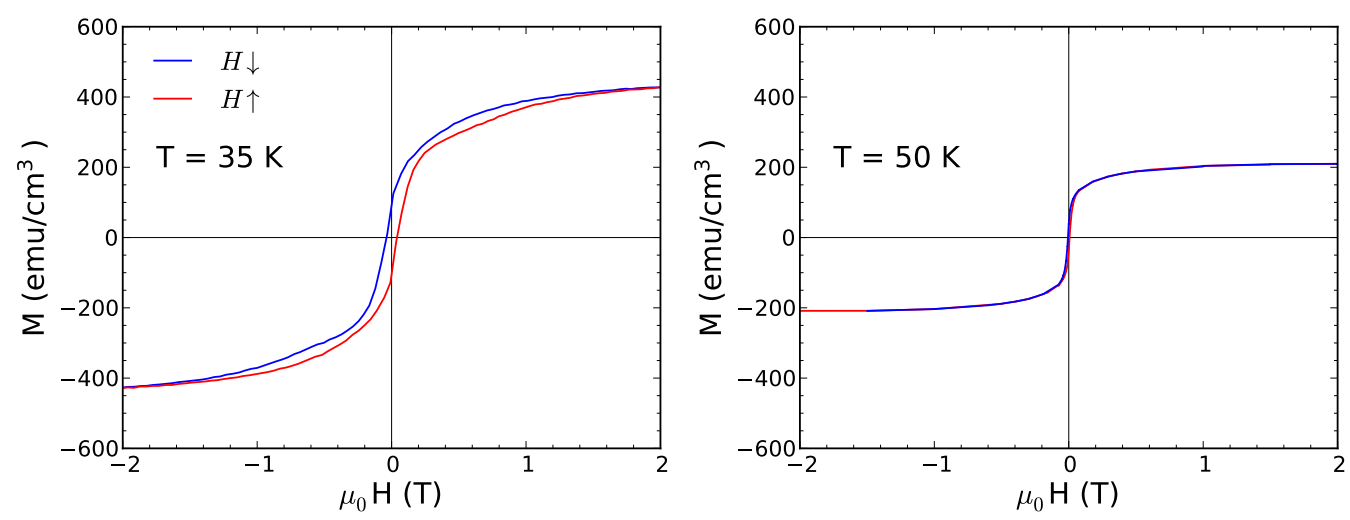

Figure 6.4: Superlattice hysteresis loops measured at (a) $T=35 \mathrm{~K}$, which shows positive $M_{R}$ and $H_{C}$ and at (b) $T=50 \mathrm{~K}$ which shows the hysteresis resembling a homogeneous GdN film.

process is symmetric upon increasing the field from this point.

We summarize the process described in Figure 6.3 as taking part in two steps. The first step is the GdN layers form a twisted magnetization phase as the field is decreased from $6 \mathrm{~T}$, eventually fully reversing at some small negative field. This results in the negative $M_{R}$. The second step occurs between about -1 and $-4 \mathrm{~T}$, where the $\mathrm{NdN}$ forms a twisted phase and slowly reverses, resulting in the "hump" in the blue magnetization curve between -4 and $-1 \mathrm{~T}$. These two processes are due to the competition between the Zeeman coupling, interface coupling, and anisotropy.

Figure 6.4 shows additional hysteresis loops measured at $T=35 \mathrm{~K}$ (with fields up to $8 \mathrm{~T}$, via VSM measurements) and $50 \mathrm{~K}$. At $35 \mathrm{~K}$, we notice that $M_{R}$ is positive, signalling that the average GdN alignment does not reverse until after the field changes sign. There is again a "hump" feature in the magnetization, occurring between -1.5 and $-0.3 \mathrm{~T}$ when the field is decreased. This suggests the NdN is undergoing magnetization reversal via a twisted phase in this region. At $35 \mathrm{~K}$ it is clear that the $\mathrm{NdN}$ has a reduced anisotropy and magnetization and is not able to effectively pin the GdN through interface exchange, but is still influenced by the interface exchange with GdN. Figure 6.4(b) shows the $M-H$ loop at $50 \mathrm{~K}$, above the NdN $T_{C}$. It resembles the hysteresis loop of a homogeneous GdN film. 

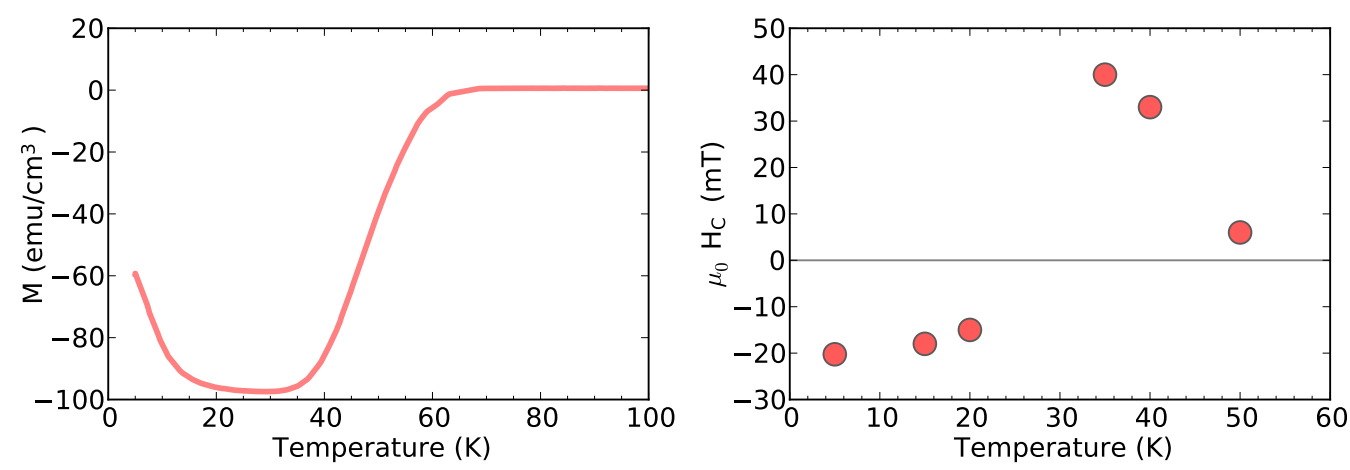

Figure 6.5: (a) Temperature dependent remanent magnetization after fieldcooling in $+6 \mathrm{~T}$. (b) Coercive field $H_{C}$ versus temperature for the superlattice.

These hysteresis measurements show that with increasing temperature the NdN anisotropy will vary strongly as $T$ approaches $40 \mathrm{~K}$, and the $\mathrm{NdN}$ will not be able to act as a rigid pinning layer for the GdN. To better understand this temperature dependence, we turn to new temperature dependent magnetization measurements. Figure 6.5(a) shows the temperature dependent remanent magnetization of the superlattice. The curve was obtained by field-cooing in a $6 \mathrm{~T}$ field in order to fully saturate the magnetization of both layers. After the field cooling process, the field was reduced to zero. The curve thus represents the remanent magnetization as a function of increasing temperature.

One immediately recognizes that the magnetization in Figure 6.5(a) is negative at $5 \mathrm{~K}$, which is at first sight surprising, however this agrees with the negative value of $M_{R}$ in Figure 6.3 when the field is decreasing. In the large $6 \mathrm{~T}$ field, the GdN and NdN should be fully saturated, and when the field is reduced to zero, the $\mathrm{NdN}$ should remain largely aligned due to its large coercive field. The GdN layer magnetization, on the other hand, with a two orders-of-magnitude smaller coercive field, begins to reverse due to interface exchange with the rigid NdN layers.

The decreasing magnetization as the temperature rises from 5 to $20 \mathrm{~K}$ is a result of the inner GdN magnetization reversing as the GdN anisotropy energy diminishes. From $20 \mathrm{~K}$ to $40 \mathrm{~K}$ the flat slope implies that the GdN magnetization is largely aligned by interface exchange, as there is no Zeeman coupling competing with the interface exchange. At about $40 \mathrm{~K}$ the bulk NdN is no 
longer in the ordered phase, while the GdN however remains ferromagnetic. Above $40 \mathrm{~K}$ the negative magnetization rapidly approaches zero as thermal fluctuations reduce the GdN alignment until the GdN $T_{C}$ is reached. This is clear evidence that the $\mathrm{GdN}$ is responsible for the negative remanent field in Figure 6.3.

We also plot $H_{C}$ as a function of temperature, shown in Figure 6.5(b), where the data has been extracted from additional hysteresis measurements. Here the transition from a negative to positive $H_{C}$ occurs between 20 and $35 \mathrm{~K}$, coinciding with the temperature of the sign change of $M_{R}$. The negative $H_{C}$ is of course a consequence of the GdN being reversed, thus above $35 \mathrm{~K}$ the GdN is aligned parallel to the field. At $50 \mathrm{~K}$, above the $\mathrm{NdN} T_{C}$, we see that the small $\approx 6 \mathrm{mT}$ coercive field is close to that expected from GdN alone, while the maximum $40 \mathrm{mT}$ coercive field occurs at $35 \mathrm{~K}$, while the NdN is still in an ordered phase.

Based on this magnetization data it is clear that the large NdN anisotropy, orbital dominant moment, and the ferromagnetic exchange coupling together result in a complex magnetic system where both NdN and GdN layers form twisted phases. At small fields, the GdN magnetization is dominated by interface exchange which reverses it before the field changes sign. The NdN is however prevented from reversing at its typical bulk $H_{C}$ value due to this interface exchange, and only reverse very gradually via a twisted phase, fully reversing only in fields over $5 \mathrm{~T}$. In the next section we explore the $\mathrm{NdN}$ and GdN layer magnetization separately via the element specific XMCD technique, which will give us indispensable insight into this complex magnetic system.

\subsection{XMCD Investigation}

In this section we make use of the element specific nature of XMCD to shed light on the individual behavior of the GdN and NdN layers in the superlattice. The XMCD is measured at the $\mathrm{Nd} \mathrm{L}_{2}$ and $\mathrm{Gd} \mathrm{L}_{2,3}$ edges, which is in the hard $\mathrm{X}$-ray regime, with energies exceeding $7 \mathrm{keV}$. At these energies, the absorption cross section is relatively low, precluding the possibility of studying single interfaces. Superlattice structures are then necessary to increase the signal by 
effectively multiplying the number of interfaces. Here we measure the same superlattice sample which was measured in the previous section, allowing for direct comparisons to be made.

\subsubsection{XMCD experimental details}

The XMCD was measured at beamline 39XU at the SPring- 8 synchrotron in Japan. The spectra were taken with the X-rays and the magnetic field co-linear and at an angle of $18^{\circ}$ from grazing incidence. The experiment took place in fields of up to $1.2 \mathrm{~T}$, and temperatures down to $16 \mathrm{~K}$, though most measurements took place at $30 \mathrm{~K}$. The XMCD was measured at a fixed magnetic field while the x-ray polarization was switched. The dichroic signal is then given by the difference between antiparallel and parallel alignments of the helicity and magnetic field. The detection scheme was partial fluorescence yield (PFY) as discussed in Chapter 3. In the PFY scheme, the $\mathrm{L}_{\alpha 1}$ fluorescence is measured when probing the $\mathrm{Nd}$ or $\mathrm{Gd} \mathrm{L}_{3}$ edge, while the $\mathrm{L}_{\beta_{1}}$ emission line is measured at the $\mathrm{Nd}$ or $\mathrm{Gd} \mathrm{L}_{2}$ edge. This method however eliminates the possibility of observing quadrupole transitions $(2 p \rightarrow 4 f)$ which directly probe the $4 f$ electrons, as the $\mathrm{L}_{\alpha_{1}, \beta_{1}}$ emission lines involve the $3 d$ states and not the $4 f$. Because fluorescence has a long mean free path of well over $1000 \mathrm{~nm}$ at these emission line energies, the measured XMCD represents the average signal from all the superlattice layers of one composition.

The XMCD spectra were normalized to the edge-jump measured from the XAS. A correction to the XMCD spectra was necessary at the Nd and Gd edges to account for a small imbalance between the degree of left and right circular polarization of the incident X-rays. To account for this, samples were field cooled from room temperature down to $16 \mathrm{~K}$ in both $\pm 1.2 \mathrm{~T}$. The sum of these two XMCD spectra should in principle yield zero for equal amounts of left and right polarized X-rays, however a small signal proportional to the absorption spectrum was present. This small background is then subtracted from the XMCD spectra. Figure 6.6 shows the raw and corrected spectra as well as the background signal, proportional to the XAS at the $\mathrm{Gd} \mathrm{L}_{2}$ and $\mathrm{Nd}$ $\mathrm{L}_{2}$ edges. The $\mathrm{GdN}_{2}$ edge requires only a small correction due to the large 

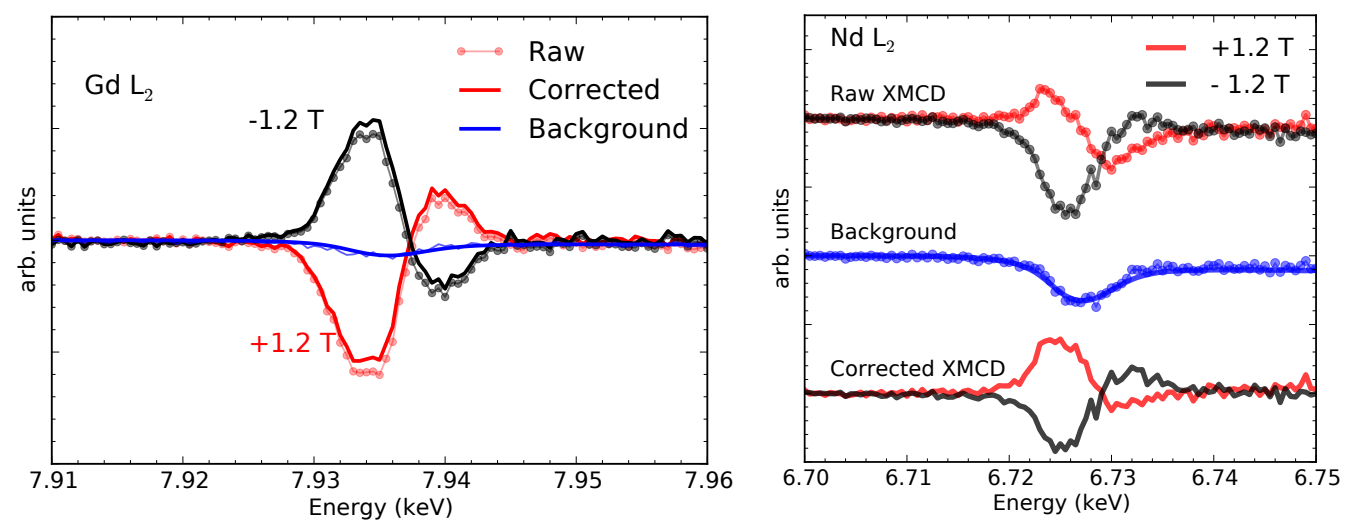

Figure 6.6: XMCD signal correction at the $\mathrm{Gd} \mathrm{L}_{2}$ edge (a) and $\mathrm{Nd} \mathrm{L}_{2}$ edge (b). XMCD spectra were measured at $16 \mathrm{~K}$ after field-cooling in $\pm 1.2 \mathrm{~T}$. The background signal is proportional to the XAS signal, and is subtracted to reveal the actual XMCD signal.

amplitude of the $\mathrm{XMCD}$, while the $\mathrm{NdN} \mathrm{L}_{2}$ is significantly distorted before correction.

\subsection{2 $\mathrm{Gd} \mathrm{L}_{2,3}$ and $\mathrm{Nd} \mathrm{L}_{2}$ edges}

We first look at the superlattice $\mathrm{Gd} \mathrm{L}_{2}$ and $\mathrm{L}_{3}$ edges, shown in Figure 6.7, which shows the XAS and XMCD spectra, taken in a field of $1.2 \mathrm{~T}$ at $16 \mathrm{~K}$, with a $30 \mathrm{~K}$ spectra also measured at the $\mathrm{L}_{2}$ edge. The sign of the XMCD spectra agree with those in studies on GdN carried out by Leuenberger et al. [103], and agree with the results of the $\mathrm{SmN} / \mathrm{GdN}$ superlattice in Chapter 5. This Gd XMCD sign means that the GdN magnetic moment (i.e. spin moment) is aligned parallel to the field. This is expected, as the large $7 \mu_{B} / \mathrm{Gd}^{3+}$ moment leads to a strong Zeeman coupling. At $30 \mathrm{~K}$ the $\mathrm{Gd} \mathrm{L}_{2}$ maintains its alignment, with slightly reduced magnitude.

We check the NdN alignment by turning to the $\mathrm{NdN} \mathrm{L}_{2}$ edge, shown in Figure 6.8. This figure shows the $\mathrm{L}_{2} \mathrm{XAS}$ and XMCD for the NdN/GdN superlattice and for the homogeneous NdN sample as well, for comparison. The $\mathrm{L}_{3}$ for the superlattice is not shown as it is too weak to clearly resolve any structure. In the figure we observe that the $\mathrm{Nd} \mathrm{L}_{2}$ is of the same sign for the superlattice and homogeneous sample, though the intensity is a factor of 

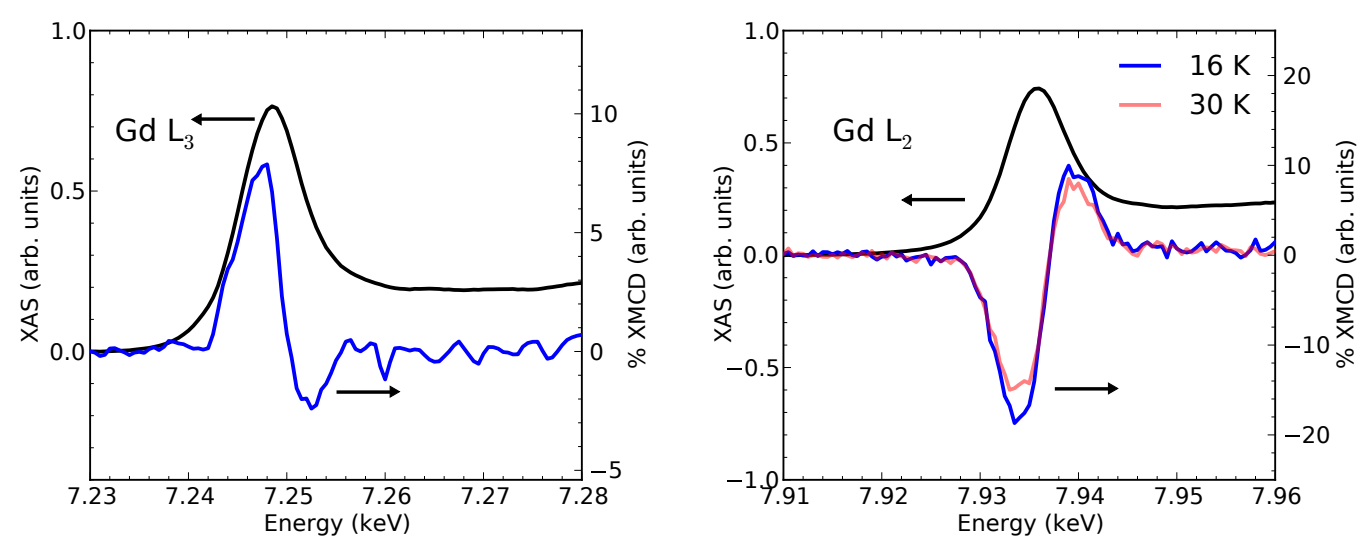

Figure 6.7: XAS and XMCD spectra from the NdN/GdN superlattice at (a) the $\mathrm{Gd} \mathrm{L}_{3}$ edge with $T=16 \mathrm{~K}$, and (b) the $\mathrm{Gd} \mathrm{L}_{2}$ edge with $T=16 \& 30 \mathrm{~K}$. The spectra were measured after field cooling in $1.2 \mathrm{~T}$.
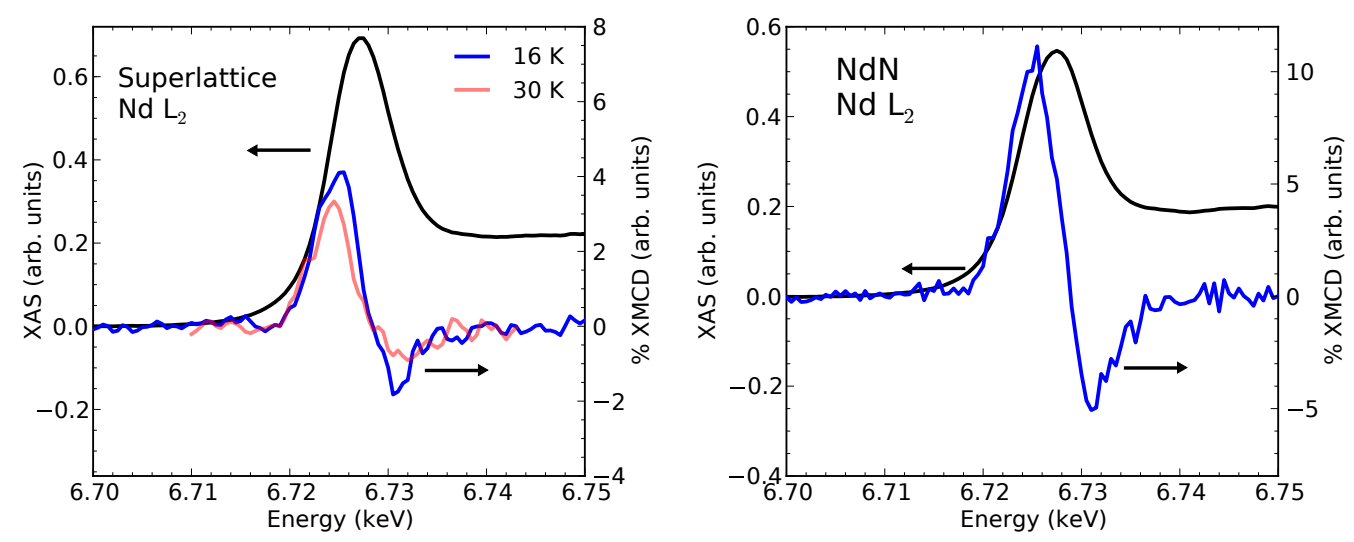

Figure 6.8: $\mathrm{Nd} \mathrm{L}_{2}$ edge XAS and XMCD spectra for (a) the NdN/GdN superlattice at $16 \mathrm{~K}$ and $30 \mathrm{~K}$ and (b) the homogeneous NdN sample at $16 \mathrm{~K}$, both after field cooling and measuring in $1.2 \mathrm{~T}$.

two smaller in the superlattice. The sign of the $\mathrm{Nd} \mathrm{L}_{2} \mathrm{XMCD}$ in both samples agrees with that of the $\mathrm{Sm} \mathrm{L}_{2}$ XMCD in homogeneous $\mathrm{SmN}$ shown in Chapter 5. This sign of the $\mathrm{Sm}_{2} \mathrm{XMCD}$ was shown to correspond to the orbital dominant magnetization in Ref. [19]. The sign difference between the $\mathrm{Nd}_{2}$ and $\mathrm{Gd} \mathrm{L}_{2}$ edge XMCD also confirms the orbital dominant NdN moment.

We can then conclude that the superlattice NdN layers have their average magnetization parallel to the field at $15 \mathrm{~K}$ and $1.2 \mathrm{~T}$. This is in contrast to 
the $\mathrm{SmN} / \mathrm{GdN}$ superlattice in Chapter 5, which showed the SmN layers had their magnetization antiparallel to the external field due to the exchange coupling with GdN. The difference can be explained by 1) the much large Zeeman coupling of $\mathrm{NdN}$ and 2) the thicker $\mathrm{NdN}$ layers (10 $\mathrm{nm} \mathrm{NdN}$ layers versus 1.5 $\mathrm{nm} \mathrm{SmN}$ layers). The reduction in the $\mathrm{Nd} \mathrm{L}_{2}$ signal by a factor of two in the superlattice suggests that the inner Nd ions in the NdN layers are Zeeman dominated, while the interface adjacent layers have their magnetization reversed by exchange with GdN. The result is a reduction in the average magnetization in the NdN superlattice layers compared to bulk NdN.

To gain insight more into the magnetic alignment of the GdN and NdN layers we turn to field-dependent XMCD measurements in the following section. 


\subsubsection{XMCD hysteresis}

In this section we examine the field dependence of the XMCD at the Gd $\mathrm{L}_{2}$ and $\mathrm{Nd} \mathrm{L}_{2}$ edges in the superlattice, shown in Figure 6.9. The temperature was fixed at $30 \mathrm{~K}$ for these measurements, and the applied field extended to $\mu_{0} H= \pm 1.2 \mathrm{~T}$. The hysteresis loops were determined by fitting each spectrum to the XMCD spectrum measured at 1.2 T. The sign of the hysteresis was chosen to follow that of the magnetization: while the most prominent peak in the $\mathrm{Gd} \mathrm{L}_{2} \mathrm{XMCD}$ is negative at $1.2 \mathrm{~T}$, the magnetization is positive, thus the $\mathrm{Gd} \mathrm{L}_{2}$ hysteresis was scaled by -1 . For the $\mathrm{NdN}$ the sign of the most prominent $\mathrm{L}_{2}$ peak is positive at $1.2 \mathrm{~T}$, thus it does not require scaling by -1 . At the $\mathrm{Gd} \mathrm{L}_{2}$ edge only half a hysteresis loop was measured for increasing field (red), while the blue curve was obtained by inverting the red curve along both axes. A full NdN hysteresis curve was measured for both increasing and decreasing fields.

We first look at the GdN hysteresis and note both the remanent magnetization and coercive field are positive, in accordance with Figure 6.5(b), which shows that the net coercive field of the superlattice changes from negative to positive between 20 and $35 \mathrm{~K}$. From the XMCD it is clear that the GdN magnetization is aligned parallel to the field at $1.2 \mathrm{~T}$, and remains aligned at remanence, in contrast to the magnetization data taken at $5 \mathrm{~K}$, where the GdN magnetization changes sign before the field reaches zero. In the magnetization data at $5 \mathrm{~K}$, it is the GdN-NdN exchange which causes the GdN magnetization to invert its sign. This implies that in the current situation, the positive GdN at zero field can be explained by the GdN being only weakly influenced by the NdN-GdN interface exchange.

It is also clear that the GdN hysteresis loop self-intersects at approximately $\pm 0.1 \mathrm{~T}$ and $\pm 0.6 \mathrm{~T}$. This is a signature of exchange coupling with $\mathrm{NdN}$. As the field is decreased from 1.2 T, the Zeeman coupling of the GdN weakens and the NdN exchange exerts some influence, however not enough to invert the GdN completely. One however notices that in contrast to the low temperature magnetization data of Figure 6.3, the magnetization with decreasing field (blue 


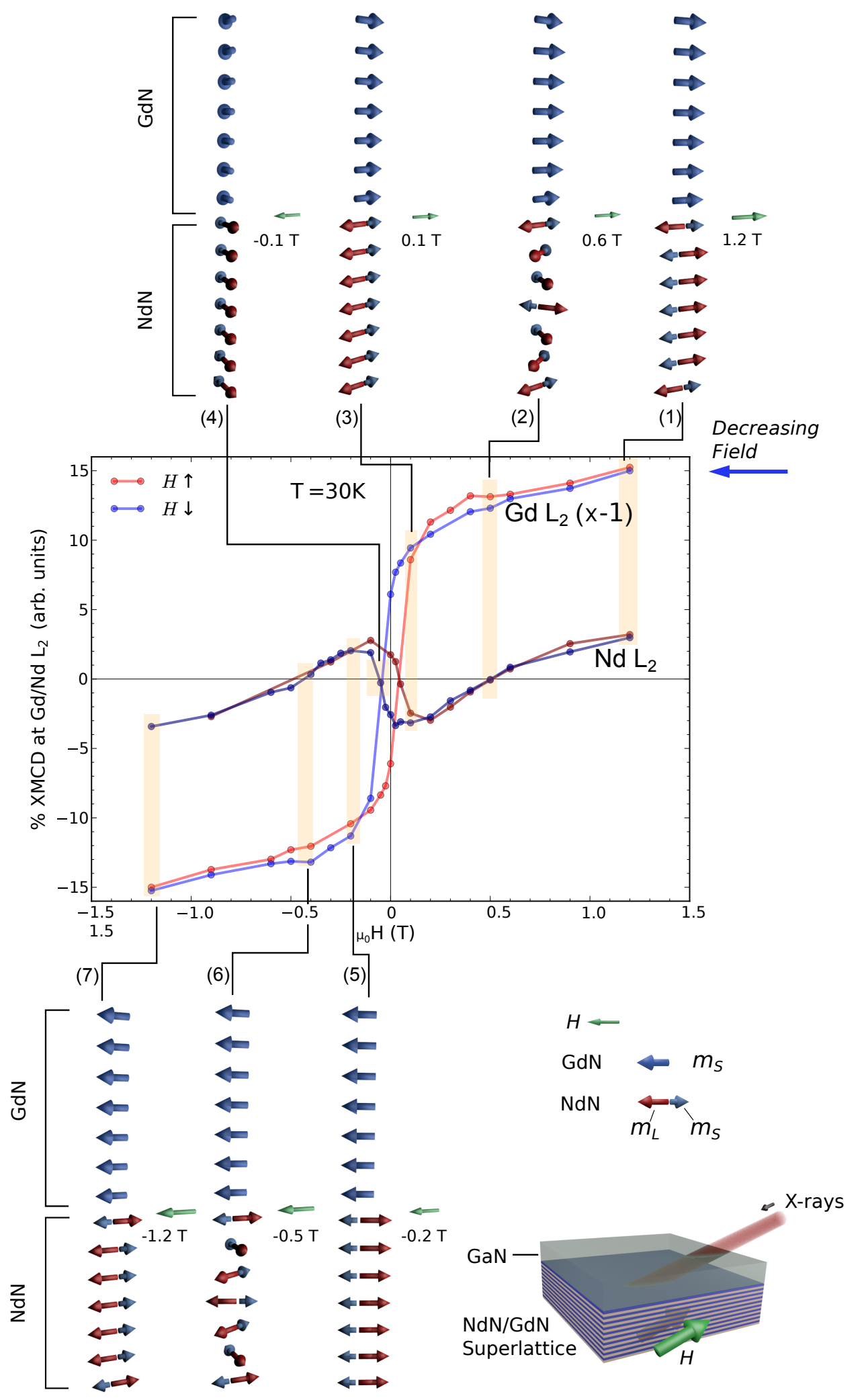

Figure 6.9: XMCD hysteresis measured at both $\mathrm{Gd}$ and $\mathrm{Nd}_{2}$ edges, where the sign has been chosen to correspond with the net magnetization within respective GdN and NdN layers. Sketches of moments correspond to the blue curves, for decreasing $H$. 
curve) is lower in the XMCD hysteresis. The shape of the $\mathrm{Gd} \mathrm{L}_{2}$ hysteresis makes it clear that it behaves largely as a homogeneous GdN sample would, with perturbations from this behavior due to the NdN exchange coupling.

We now turn to the $\mathrm{NdN}$ hysteresis, following the $\mathrm{Nd}$ alignment as the field is decreased from 1.2 T. As the field decrease, we see that the Nd signal decreases as well, becoming zero at $0.5 \mathrm{~T}$. This signals that as the NdN Zeeman coupling weakens, a twisted magnetization arising from interface exchangeZeeman competition nucleates from the interfaces deeper into NdN layers. The decreasing field increases the twist length scale, which according to the model of Chapter 5 varies as $\ell \propto 1 / \sqrt{|H|}$. The periodic boundary conditions of the superlattice mean that the twist is symmetric about the center of the $\mathrm{NdN}$ layers, and when $H=0.5 \mathrm{~T}$ the average alignment of the $\mathrm{NdN}$ in the plane of the film is zero. Below $0.5 \mathrm{~T}$ the $\mathrm{NdN}$ magnetization is dominated by interface exchange, resulting in a negative magnetization alignment with respect to the field. When the field reaches $0.1 \mathrm{~T}$ the $\mathrm{NdN}$ spin-moment is strongly coupled to the GdN by interface exchange, and the magnetization rigidly follows the GdN in the low field region where there is vanishing Zeeman coupling. The NdN reversal continues until $-0.2 \mathrm{~T}$, when the Zeeman coupling of the $\mathrm{NdN}$ is large enough to compete with the NdN-GdN exchange. A twisted phase begins to nucleate in the inner $\mathrm{NdN}$ layers as the NdN Zeeman coupling becomes stronger. The average NdN alignment passes through zero at $-0.5 \mathrm{~T}$ and then arrives in the Zeeman dominated regime where the orbital moment is parallel to $H$.

This situation is shown in the sketches in Figure 6.9, which show the spin and orbital moments of the GdN and NdN layers assuming periodic boundary conditions. The sketches are idealized to some degree, as the effect of incomplete saturation of the $\mathrm{NdN}$ and $\mathrm{GdN}$ layers have been ignored. The shape anisotropy in the thin film geometry should keep the magnetization of the film in plane, thus we have only considered rotations of the magnetization in the plane of the film, similar to a Bloch domain wall.

Figure 6.10 shows an additional $16 \mathrm{~K}$ hysteresis loop extracted from XMCD at the $\mathrm{Nd} \mathrm{L}_{2}$ edge (with the $30 \mathrm{~K}$ data also shown for comparison. The $16 \mathrm{~K}$ data shows the same characteristics as the $30 \mathrm{~K}$ measurement, though with a 


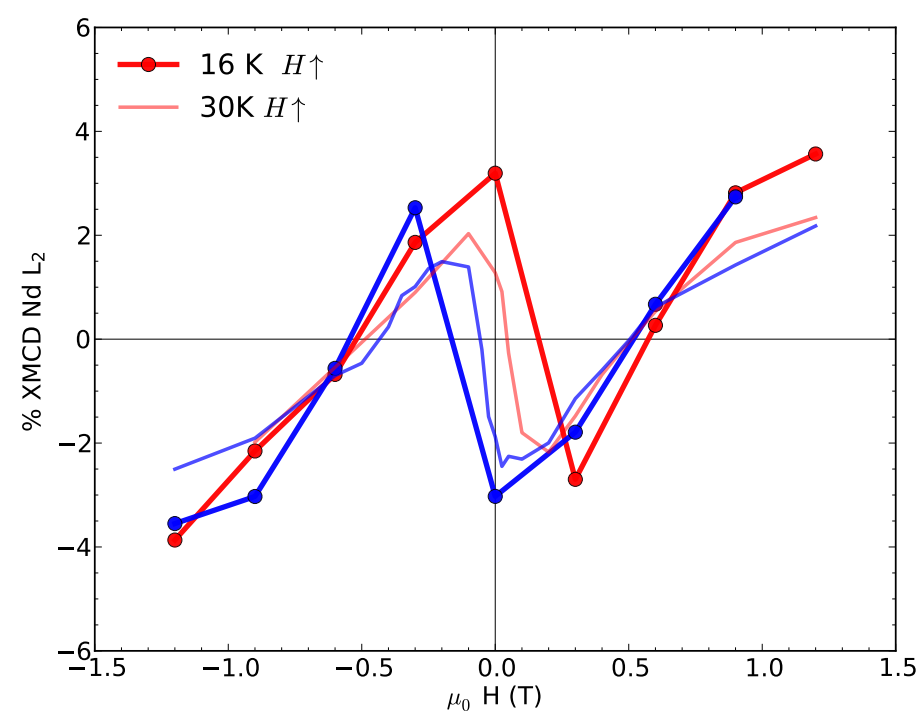

Figure 6.10: Comparison of the $\mathrm{Nd} \mathrm{L}_{2}$ hysteresis from the $\mathrm{NdN} / \mathrm{GdN}$ superlattice measured at $16 \mathrm{~K}$ and $30 \mathrm{~K}$. Red and blue curves denote measurements with $H$ increasing and decreasing, respectively.

larger amplitude expected at lower temperature.

\subsection{Discussion}

The preceding sections have clearly demonstrated that in the NdN/GdN superlattice the unique interface exchange coupling produces complex magnetic structures. The twisted phase due to exchange-Zeeman competition manifests in two ways: in the low temperature SQUID magnetization data we saw that the GdN magnetization switched first, while the NdN magnetization reversed fully in very large negative fields. In contrast, the XMCD hysteresis showed that the NdN magnetization reverses first, followed by the GdN only after the field reverses. This suggests categorization of the twisted phases as follows: a "soft phase" where the soft GdN layers pass through a twisted phase and reverse before the field does, and a "hard phase" where the hard NdN layers enter a twisted phase and reverse before the field reaches zero. The boundary between these phases depends on both temperature and the maximum applied 


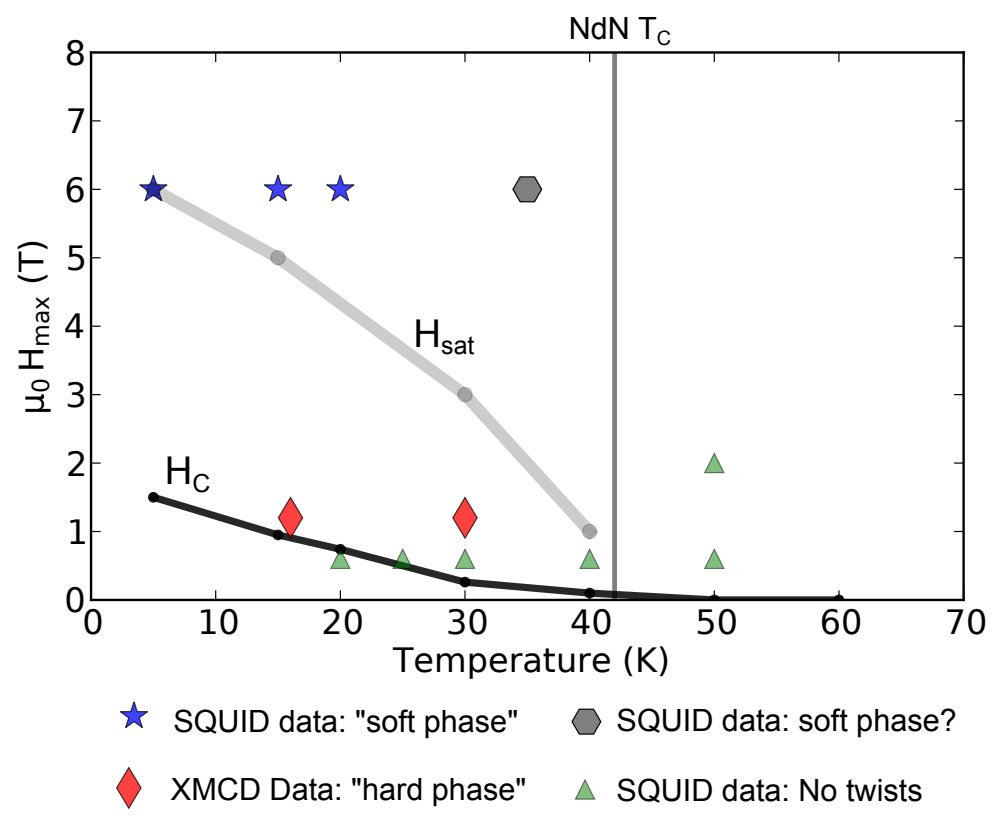

Figure 6.11: A proposed phase diagram of the twisted magnetic phases in the NdN/GdN superlattice. $H_{\text {sat }}$ represents the approximate field required to saturate the magnetization in bulk NdN. $H_{C}$ is the coercive field of $\mathrm{NdN}$.

field.

The origin of these two phases is related to the magnetic anisotropy of the $\mathrm{NdN}$ layers. At $5 \mathrm{~K}$ the coercive field is about $1.5 \mathrm{~T}$, while in $\mathrm{GdN}$ it is of order $0.01 \mathrm{~T}$. Because of the rather low $T_{C}$ of $\mathrm{NdN}$, the anisotropy energy decreases significantly as the temperature is increased. For the XMCD measurements at 16 and $30 \mathrm{~K}$, the NdN anisotropy is relatively small, and the NdN is dominated by the interface exchange coupling with GdN. In the $5 \mathrm{~K}$ SQUID measurements however, the $6 \mathrm{~T}$ field is large enough to fully align the NdN layers, which then remain fixed due to the large anisotropy, while the GdN layers enter a twisted phase.

Figure 6.11 shows a phase diagram taken from all available magnetization and XMCD measurements, with temperature plotted along the horizontal axis and the maximum applied field $\left(\mu_{0} H_{\max }\right)$ along the vertical axis. The soft phase, in which the GdN enters a twisted phase, is located in the upper left quadrant, with the maximum applied field at $6 \mathrm{~T}$. The three data points (blue stars) all have $M_{R}<0$, signalling the GdN layers reverse their magnetization 
before $H$ reaches zero. The grey hexagon at $35 \mathrm{~K}$ represents the hysteresis in Figure 6.11, and which shows that $M_{R}>0$, though it is only $25 \%$ of the saturation magnetization at high field. At this temperature and maximum applied field, the GdN may still enter a twisted phase, but the interface-exchange is not strong enough to produce a negative magnetization in the GdN layer. This point is then possibly in the soft phase.

The two XMCD measurements at $16 \mathrm{~K}$ and $30 \mathrm{~K}$ (red diamonds) in the hard phase are separated from the soft phase by the line $H_{\text {sat }}$ which represents the saturation field of bulk NdN, and corresponds approximately to the boundary between the soft and hard phase. It seems that a field of at least $H_{\text {sat }}$ must be applied to align the $\mathrm{NdN}$ magnetization to a sufficient degree for producing a rigid pinning layer for the GdN layers to develop a twisted phase. If the maximum applied field is much less than $H_{\text {sat }}$, the NdN magnetization is more disordered and the interface exchange coupling will decrease in energy. The GdN magnetization will consequently be dominated by Zeeman coupling.

Finally, the green triangles represent SQUID measurements which show no apparent evidence of a twisted phase. Below the $\mathrm{NdN} T_{C}$, this is most likely because the maximum applied field is not large enough to align the NdN layers to any significant degree. Above the $\mathrm{NdN} T_{C}$, the $\mathrm{GdN}$ is responsible for the ordering. Interface exchange may take place with the GdN, but above the $\mathrm{NdN} T_{C}$ there is no intra-layer NdN exchange to support any twisted phase, even in large fields. In this case one expects only the atomic layers adjacent to the GdN to be exchange coupled.

\subsection{Conclusion and Outlook}

The combination of lab based magnetization measurements and the element specific tool of XMCD paint a complex picture of the NdN/GdN system, which we have explored for the first time. There are two primary conclusions we make in regards to the NdN/GdN system. First, there is the appearance of two different kinds of twisted phases. The soft phase where the GdN enters a twisted phase upon decreasing the field from saturation, and the hard phase where the NdN layers enter a twisted phase when decreasing the field from saturation, 


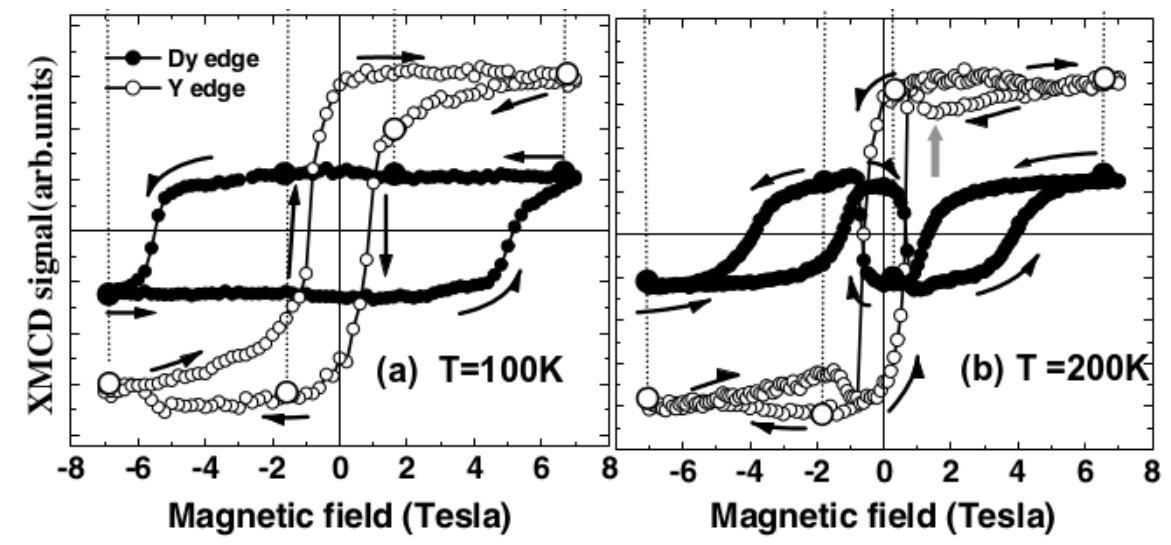

Figure 6.12: $\mathrm{XMCD}$ at the $\mathrm{Dy}$ and $\mathrm{Y} \mathrm{L}_{3}$ edge in a $\mathrm{DyFe}_{2} / \mathrm{YFe}_{2}$ superlattice, from Dumesnil et al., Ref. [104]. $\mathrm{YFe}_{2}$ is a weak ferrimagnet and $\mathrm{DyFe}_{2}$ is a hard ferrimagnet. (a) The hard phase XMCD derived hysteresis at $100 \mathrm{~K}$, (b) soft phase XMCD derived hysteresis at $200 \mathrm{~K}$.

while the GdN layers behave in a more usual manner. The second conclusion is that this $\mathrm{NdN} / \mathrm{GdN}$ system confirms our model of an orbital-driven twisted phase described in Chapter 5 for the $\mathrm{SmN} / \mathrm{GdN}$ system. The orbital-dominant magnetization of $\mathrm{SmN}$ and $\mathrm{NdN}$ make this unusual asymmetry between ferromagnetic interface exchange and Zeeman coupling possible, and drives the competition resulting in the twisted phases.

Further work is needed to better understand the NdN/GdN system. In particular, XMCD experiments with large fields and lower temperatures are necessary to better compare with the lab based magnetometry. The sketches of the GdN and NdN magnetizations may have more complex behavior than we have sketched above. To obtain a better picture of the magnetic structure detailed calculations will need to be made.

We note there exist similar types of twisted phases in the metallic exchangespring $\mathrm{DyFe}_{2} / \mathrm{YFe}_{2}$, where the $\mathrm{DyFe}_{2}$ is a hard ferrimagnet with the Dy moment dominant, and $\mathrm{YFe}_{2}$ is a weak ferrimagnet with the Fe moment dominant [104, 105]. Dy, as a heavy rare-earth, has the spin and orbital moments parallel. In this case, there is ferromagnetic Fe-Fe exchange, however this exchange results in an antiferromagnetic coupling of the net magnetizations. This leads to similar hard and soft twisted phases as in the NdN/GdN superlattice sys- 
tem, shown in Figure 6.12 for a $\mathrm{DyFe}_{2} / \mathrm{YFe}_{2}$ superlattice (From Dumesnil et al [104]. The figures show Dy and $\mathrm{Y} \mathrm{L}_{3}$ edge XMCD derived hysteresis loops. The soft phase (Fig. 6.12(b)) shows very similar behavior to the $16 \mathrm{~K} \mathrm{XMCD}$ data in Figure 6.9.

The accrued knowledge from the study of the complex behavior of $\mathrm{DyFe} 2 / \mathrm{YFe}_{2}$ will doubtless aid in future investigations of $\mathrm{NdN} / \mathrm{GdN}$ and $\mathrm{SmN} / \mathrm{GdN}$ heterostructures. However we note again the important distinction: the rare-earth nitride superlattices are intrinsic ferromagnetic semiconductors. The strong spin-orbit coupling keeps the spin and orbital moments fixed relative to each other even when strong exchange fields are present, as was shown in the calculations of Chapter 4 and 5. It may be interesting to study the magnetoresistance of REN heterostructures with twisted phases. There are some reports of giant magnetoresistance due to twisted phases in conventional exchange spring systems such as $\mathrm{DyFe}_{2} / \mathrm{YFe}_{3}$ [106]. The effect of such an unconventional "domain wall" in the REN superlattice may yield interesting results. 


\section{Chapter 7}

\section{Conclusions and Outlook}

\section{$7.1 \quad$ Results}

In this thesis we have investigated the magnetic properties of $\mathrm{SmN}$ and $\mathrm{NdN}$ in detail, and advanced our understanding of these materials in two major ways. We first investigated the fundamental quantity of the magnetic moment in $\mathrm{SmN}$ and $\mathrm{NdN}$. We showed that crystal field calculations, when properly carried out by including excited $J$-multiplets, lead to good agreement with experimental data. Our results differ from previous band-structure calculations using advanced density-functional theory methods, which predicted nearly maximal values of the orbital and spin moments in $\mathrm{NdN}$ and $\mathrm{SmN}$, while our results showed that the crystal field effects in SmN and NdN cause significant quenching of the orbital and spin moments. This partial quenching has also been observed in some heavy rare-earth nitrides such as DyN, ErN, and HoN, adding support to our finding that quenching plays an important role in the RENs, in contrast to previous calculations.

In NdN, good agreement was found with the published NdN data from Schumacher et al. and the crystal field calculations, however the ordered moment and larger $T_{C}$ of thin-film NdN could not be adequately explained. Likely causes of the smaller ordered moment and larger $T_{C}$ in the NdN thin-film are substrate-induced strain and the effects of doping due to nitrogen vacancies. The effect of nitrogen vacancies are known to modify the Curie temperature in GdN due to the formation of magnetic polarons, suggesting the possibility 


\section{Chapter 7 Conclusions and Outlook}

of a similar effect occurring in $\mathrm{NdN}$.

The second major result is the observation of unconventional twisted magnetization phases in $\mathrm{SmN}$ and NdN films when coupled to GdN. These twisted phases were shown to arise from competition between ferromagnetic interface exchange (acting only on spin) and the orbital-dominant Zeeman coupling of $\mathrm{SmN}$ and NdN. When $\mathrm{SmN}$ is exchange coupled to GdN (with a purely spin moment) an external field keeps GdN fixed while the Zeeman coupling tends to rotate the $\mathrm{SmN}$ magnetization, this competition results in the twisted phase. Using the element-specific technique of XMCD, we observed a twisted SmN magnetization in a $\mathrm{SmN} / \mathrm{GdN}$ bilayer. An analytical model was developed to explain the XMCD at the M-edge in a natural way. We furthermore showed that the crystal-field calculations could explain the enhanced spin and orbital alignment at the interface $\mathrm{SmN}$ layers, lending further support to the technique. In a NdN/GdN superlattice magnetization and XMCD measurements unambiguously revealed twisted phases occurring in both NdN and GdN, depending on the temperature and maximum applied field. The observation of the twisted phase in the $\mathrm{NdN} / \mathrm{GdN}$ system also confirms the findings in the SmN/GdN system, establishing that the orbital-dominant Zeeman coupling competes with ferromagnetic interface exchange with GdN to drive the phase.

Systems exhibiting similar twisted phases either rely on large anisotropy differences between materials, or have antiferromagnetic exchange coupling at interfaces to produce competition interactions resulting in a twisted phase. All of these systems are 1) metallic and 2) spin-dominant, establishing that twisted phases in rare-earth nitride heterostructures are quite unconventional.

\subsection{Future Outlook}

The good agreement between the crystal field calculations and experiment for SmN and NdN suggest that calculations could be applied to other RENs to good effect. The next to study would be the latter half of the REN series, where DyN, ErN, and HoN have detailed experimental studies of their magnetic properties [6, 70]. It would be useful to determine any trends in the crystal field parameters across the series. Ultimately, a knowledge of the spin 
and orbital moments would benefit other theoretical calculations that depend on these values. Another useful effort would be to revisit band structure calculations in the RENs in light of the findings in this thesis. More accurate predictions of the band structures and the resulting spin and orbital moments would greatly aid in the interpretation of a vast range of experiments. Finally, it would be interesting to see if advanced band structure calculations could provide some insight into the crystal field model in the RENs, and perhaps some correspondence between the two approaches could be made.

There are many possible directions to take in studying twisted phases in REN heterostructures. The obvious next steps are to conduct more XMCD experiments on the $\mathrm{SmN} / \mathrm{GdN}$ and $\mathrm{NdN} / \mathrm{GdN}$ systems. In-situ growth at an M-edge XMCD beamline would allow for more detailed depth-dependent studies. Further L-edge XMCD studies in larger fields and lower temperatures are already being planned for NdN/GdN superlattices. Depth sensitive neutron reflectivity measurements should also provide more detailed information. These additional measurements in a variety of temperatures, fields, and geometries should provide a better picture of the twisted structure. Measurements to determine the anisotropy contributions would also be useful and aid in modelling these phases.

The ability to substitute rare-earth ions may also lead to interesting results. Substituting Gd ions in $\mathrm{SmN}$ and $\mathrm{NdN}$ should reduce the overall magnetization while also modifying the exchange, which could allow the ability to tune the length scales of the twisted phases independently of the applied field. These twisted phases can thus be viewed as engineered domain-walls with tunable domain-wall widths. Current-driven domain wall motion is an active field, so it may be interesting to explore this area in the RENs. An open question is the transport characteristics through these twisted phases, which may show some interesting behavior. There have been reports of giant magnetoresistance in metallic exchange-spring systems [106] for example, so it seems worth pursuing similar experiments in the RENs, in addition to tunneling magnetoresistance. Because the RENs can be doped to achieve a wide range of carrier concentrations, the conductivity can be tuned to the desired application, while the magnetic properties are relatively unaffected. There are thus many unexplored 
Chapter 7 Conclusions and Outlook

avenues worth pursuing in REN heterostructures. 
7.2 Future Outlook 
Chapter 7 Conclusions and Outlook 
Appendices 



\section{Appendix A}

\section{Matrix Elements of Irreducible Tensor Operators}

\section{A.1 Irreducible Tensor Operators}

The Wigner-Eckart theorem provides a useful relationship for matrix elements of tensor operators with respect to total angular moment eigenstates $\left\{\left|L S J M_{J}\right\rangle\right\}$ (see, e.g. Refs $[32,107,108]$ ). An irreducible tensor operator is fully defined by its commutation relations with components of the total angular moment operator J. First we define the following angular moment "ladder" operators:

$$
J_{ \pm}=J_{x} \pm i J_{y}
$$

These operators have the following commutation relations (the commutator is $[A, B]=A B-B A)$ :

$$
\left[J_{ \pm}, J_{\mp}\right]= \pm 2 J_{z} \quad \text { and } \quad\left[J_{z}, J_{ \pm}\right]= \pm J_{ \pm}
$$

An irreducible tensor operator can now be defined as an object $\mathcal{T}_{q}^{(k)}$ which satisfies the following commutation relations:

$$
\begin{aligned}
{\left[J_{z}, \mathcal{T}_{q}^{(k)}\right] } & =q \mathcal{T}_{q}^{(k)} \\
{\left[J_{ \pm}, \mathcal{T}_{q}^{(k)}\right] } & =\sqrt{k(k+1)-q(q \pm 1)} \mathcal{T}_{q \pm 1}^{(k)}
\end{aligned}
$$


where the rank $k$, of the operator refers to the number of independent components, equal to $2 k+1$, and $q$ indexes these components, with $q=-k,-k+$ $1, \ldots, k-1, k$. For example $k=0$ corresponds to a scalar operator (one component), $k=1$ a vector operator with components $q=-1,0,1$, and $k \geqslant 2$ corresponds to tensor operators.

The term "irreducible" corresponds to the fact that $\mathcal{T}_{q}^{(k)}$ transforms under rotations as a spherical harmonic of rank $k$, and cannot be decomposed into a sum of lower rank operators (in contrast, Cartesian tensor operators are not necessarily irreducible).

\section{A.2 The Wigner-Eckart Theorem}

The statement of the Wigner-Eckart theorem is made for angular momentum eigenstates $|\alpha, J M\rangle$ where $\alpha$ indicates other quantum numbers (such as $L, S$, and the radial quantum number $n$ ). The theorem tells us how to calculate matrix elements of an irreducible tensor operator $\mathcal{T}_{q}^{(k)}$ :

$$
\left\langle\alpha J M\left|\mathcal{T}_{q}^{(k)}\right| \alpha^{\prime} J^{\prime} M^{\prime}\right\rangle=(-1)^{J-M}\left(\begin{array}{ccc}
J & k & J^{\prime} \\
-M & q & M^{\prime}
\end{array}\right)\left\langle\alpha J\left\|\mathcal{T}^{(k)}\right\| \alpha^{\prime} J^{\prime}\right\rangle
$$

where the large parentheses denotes the Wigner-3j symbol and the reduced matrix element $\left\langle\alpha J\left\|\mathcal{T}^{(k)}\right\| \alpha^{\prime} J^{\prime}\right\rangle$ is independent of $M$ and $M^{\prime}$. The $3 j$ symbol is defined in terms of the Clebsch-Gordan coefficient by

$$
\left(\begin{array}{ccc}
j_{1} & j_{2} & J \\
m_{1} & m_{2} & -M
\end{array}\right)=(-1)^{M+j_{1}-j_{2}} \frac{\left\langle j_{1} j_{2} m_{1} m_{2} \mid j_{1} j_{2}: J M\right\rangle}{\sqrt{2 j+1}},
$$

where $\boldsymbol{j}_{1}+\boldsymbol{j}_{2}=\boldsymbol{J}$ and $m_{1}+m_{2}=M$. The Clebsch-Gordan coefficients form the unitary transformation between the $\left\{\left|j_{1} j_{2} m_{1} m_{2}\right\rangle\right\}$ basis and $\left\{\left|j_{1} j_{2}: J M\right\rangle\right\}$ basis. For example,

$$
\left|j_{1} j_{2}: J M\right\rangle=\sum_{m_{1}} \sum_{m_{2}}\left\langle j_{1} j_{2} m_{1} m_{2} \mid j_{1} j_{2}: J M\right\rangle\left|j_{1} j_{2} m_{1} m_{2}\right\rangle
$$


In terms of Clebsch-Gordan coefficients the Wigner-Eckart theorem reads

$$
\left\langle\alpha J M\left|\mathcal{T}_{q}^{(k)}\right| \alpha^{\prime} J^{\prime} M^{\prime}\right\rangle=\frac{\left\langle J^{\prime} k M^{\prime} q \mid J M\right\rangle}{\sqrt{2 J+1}}\left\langle\alpha, J\left\|\mathcal{T}^{(k)}\right\| \alpha^{\prime}, J^{\prime}\right\rangle
$$

The meaning of the Wigner-Eckart theorem (A.5) is that it separates the geometrical factors (depending on $M, M^{\prime}$ ) out from the part describing the physical operator, which is described by the reduced matrix element. For vector operators $k=1$, if $J^{\prime}=J$, the Wigner-Eckart reduces to the simplified projection theorem:

$$
\left\langle\alpha, J M\left|\mathcal{T}_{q}\right| \alpha^{\prime}, J M^{\prime}\right\rangle=\frac{\left\langle\alpha, J M|\mathcal{T} \cdot \mathbf{J}| \alpha^{\prime}, J M\right\rangle}{J(J+1)}\left\langle J M\left|J_{q}\right| J M^{\prime}\right\rangle
$$

For example, setting $\mathcal{T}=\mathbf{L}$, for $L_{z}=L_{0}$ the projection theorem gives

$$
\begin{aligned}
\left\langle\alpha, J M\left|L_{z}\right| \alpha^{\prime}, J M^{\prime}\right\rangle & =\frac{\left\langle\alpha, J M\left|\mathbf{L}^{2}+\frac{1}{2}\left(\mathbf{J}^{2}-\mathbf{L}^{2}-\mathbf{S}^{2}\right)\right| \alpha^{\prime}, J M^{\prime}\right\rangle}{J(J+1)}\left\langle J M\left|J_{z}\right| J M^{\prime}\right\rangle \\
& =\frac{1}{2}+\frac{1}{2} \frac{L(L+1)-S(S+1)}{J(J+1)}\left\langle J M\left|J_{z}\right| J M^{\prime}\right\rangle
\end{aligned}
$$

Similarly for $S_{z}=S_{0}$

$$
\begin{aligned}
\left\langle\alpha, J M\left|S_{z}\right| \alpha^{\prime}, J M^{\prime}\right\rangle & =\frac{\left\langle\alpha, J M\left|\mathbf{S}^{2}+\frac{1}{2}\left(\mathbf{J}^{2}-\mathbf{L}^{2}-\mathbf{S}^{2}\right)\right| \alpha^{\prime}, J M^{\prime}\right\rangle}{J(J+1)}\left\langle J M\left|J_{z}\right| J M^{\prime}\right\rangle \\
& =\frac{1}{2}-\frac{1}{2} \frac{L(L+1)-S(S+1)}{J(J+1)}\left\langle J M\left|J_{z}\right| J M^{\prime}\right\rangle .
\end{aligned}
$$

Thus the operator $M_{z}=L_{z}+2 S_{z}$ can be seen to be

$$
\begin{aligned}
\left\langle\alpha, J M\left|L_{z}+2 S_{z}\right| \alpha^{\prime}, J M^{\prime}\right\rangle & =\frac{3}{2}-\frac{1}{2} \frac{L(L+1)-S(S+1)}{J(J+1)}\left\langle J M\left|J_{z}\right| J M^{\prime}\right\rangle \\
& =g_{J}\left\langle J M\left|J_{z}\right| J M^{\prime}\right\rangle
\end{aligned}
$$

where we have defined the Landé $g_{J}$-factor. From this definition it is easy to 
show that

$$
\begin{aligned}
& \left\langle\alpha, J M\left|S_{z}\right| \alpha^{\prime}, J M^{\prime}\right\rangle=\left(g_{J}-1\right)\left\langle J M\left|J_{z}\right| J M^{\prime}\right\rangle \\
& \left\langle\alpha, J M\left|L_{z}\right| \alpha^{\prime}, J M^{\prime}\right\rangle=\left(2-g_{J}\right)\left\langle J M\left|J_{z}\right| J M^{\prime}\right\rangle,
\end{aligned}
$$

which were given in Chapter 4. A simpler alternative for deriving the relation between $L_{z}+2 S_{z}$ and $J_{z}$ exists using purely geometrical considerations, and is found in many introductory quantum mechanics textbooks. The WignerEckart and projection theorems allow for the use of algebraic methods without resorting to geometrical considerations.

\section{Consequences of the Wigner-Eckart Theorem}

An important consequence of the Wigner-Eckart theorem is that matrix elements of tensor operators within a fixed $J$-multiplet (i.e. $J^{\prime}=J$ ) are proportional to each other. Given tensor operators $\mathcal{T}_{q}^{(k)}$ and $\mathcal{O}_{q}^{(k)}$, we have

$$
\frac{\left\langle\alpha, J M^{\prime}\left|\mathcal{T}_{q}^{(k)}\right| \alpha^{\prime}, J M^{\prime}\right\rangle}{\left\langle\alpha, J M^{\prime}\left|\mathcal{O}_{q}^{(k)}\right| \alpha^{\prime}, J M^{\prime}\right\rangle}=\frac{\left\langle\alpha, J\left\|\mathcal{T}^{(k)}\right\| \alpha^{\prime}, J\right\rangle}{\left\langle\alpha, J\left\|\mathcal{O}^{(k)}\right\| \alpha^{\prime}, J\right\rangle},
$$

which shows that the ratio of these operators does not depend on the geometry of the system through $M, M^{\prime}$.

More generally, given a Hamiltonian $\mathscr{H}$ which depends on parameters such as $\Lambda, J_{e x}, A_{4}, A_{6}$, if only the lowest $J$-multiplet is considered an eigenstates of $\mathscr{H}$ can be written as

$$
\left|\psi_{i}\right\rangle=\sum_{M} c_{M}^{i}|\alpha, J M\rangle
$$

where the coefficients depend on the parameters of the Hamiltonian. In this case the expectation value of a tensor operator within the state $\left|\psi_{i}\right\rangle$ is given 
by

$$
\begin{aligned}
\left\langle\psi_{i}\left|\mathcal{T}_{q}^{(k)}\right| \psi_{i}\right\rangle & =\sum_{M, M^{\prime}} c_{M}^{* i} c_{M^{\prime}}^{i}\left\langle\alpha, J M\left|\mathcal{T}_{q}^{(k)}\right| \alpha^{\prime}, J M^{\prime}\right\rangle \\
& =\left(\sum_{M, M^{\prime}} c_{M}^{* i} c_{M^{\prime}}^{i}(-1)^{J-M}\left(\begin{array}{ccc}
J & k & J \\
-M & q & M^{\prime}
\end{array}\right)\right)\left\langle\alpha J\left\|\mathcal{T}^{(k)}\right\| \alpha^{\prime} J^{\prime}\right\rangle \\
& =\mathcal{U}_{k, q}(J)\left\langle\alpha J\left\|\mathcal{T}^{(k)}\right\| \alpha^{\prime} J^{\prime}\right\rangle
\end{aligned}
$$

Thus the matrix elements of the $q$-th component of every tensor operator of rank $k$ within a single $J$ multiplet are proportional to $\mathcal{U}_{k, q}(J)$. The ratio of reduced matrix elements give the proportionality constants between operators. Varying the parameters of the Hamiltonian changes $\mathcal{U}_{k, q}(J)$, but not the ratio of tensor operators. Thus within a single $J$-multiplet the ratio of the matrix elements of any two tensor operators remains fixed.

If higher $J$-multiplets are considered, then the ratio of the matrix elements of tensor operators depend on the coefficients $c_{M}^{i}$, and thus the parameters in the Hamiltonian. In this case $\frac{\left\langle\psi_{i}\left|\mathcal{T}_{q}^{(k)}\right| \psi_{i}\right\rangle}{\left\langle\psi_{i}\left|\mathcal{O}_{q}^{(k)}\right| \psi_{i}\right\rangle}=\frac{\mathcal{U}_{k, q}(J)\left\langle\alpha J\left\|\mathcal{T}^{(k)}\right\| \alpha^{\prime} J^{\prime}\right\rangle+\mathcal{U}_{k, q}(J, J+1)\left\langle\alpha J\left\|\mathcal{T}^{(k)}\right\| \alpha^{\prime} J+1\right\rangle+\ldots}{\mathcal{U}_{k, q}(J)\left\langle\alpha J\left\|\mathcal{O}^{(k)}\right\| \alpha^{\prime} J^{\prime}\right\rangle+\mathcal{U}_{k, q}(J, J+1)\left\langle\alpha J\left\|\mathcal{O}^{(k)}\right\| \alpha^{\prime} J+1\right\rangle+\ldots}$

This is an important consideration for $\mathrm{Sm}^{3+}$, where the spin and orbital moments are antiparallel and nearly equal and magnitude, so small changes in the ratio of $\left\langle L_{z}\right\rangle /\left\langle S_{z}\right\rangle$ can change the net moment considerably.

\section{A.3 Matrix Elements Between Arbitrary Mul- tiplets}

Here we derive the matrix elements for arbitrary $J$-multiplets given in Chapter 4. We first derive Equations 4.12, the matrix element $\left\langle J M\left|L_{z}\right| J^{\prime} M^{\prime}\right\rangle$. We first 
apply the Wigner-Eckart Theorem to separate out the $M$ dependence, using the notation $L_{z}=L_{0}^{(1)}$ :

$$
\left\langle L S J M\left|L_{0}^{(1)}\right| L S J^{\prime} M^{\prime}\right\rangle=(-1)^{J-M}\left(\begin{array}{ccc}
J & k & J^{\prime} \\
-M & q & M^{\prime}
\end{array}\right)\left\langle L S J\left\|L^{(1)}\right\| L S J^{\prime}\right\rangle
$$

The calculation of $\left\langle L S J\left\|L^{(1)}\right\| L S J^{\prime}\right\rangle$ is more involved because $L^{(1)}$ is acting on only one part of a coupled system of angular momentum vectors. The derivation of this matrix element uses the Clebsch-Gordan decomposition multiple times to recouple the angular moment states, along with repeated use of the Wigner-Eckart theorem. The result involves a double sum over four ClebschGordan coefficients (or $3 j$ symbols), which more compactly expressed by a Wigner $6 j$-symbol. For a coupled system with $\boldsymbol{j}_{1}+\boldsymbol{j}_{2}=\boldsymbol{J}$, and an operator $\mathcal{T}^{(k)}(1)$ acting on $\boldsymbol{j}_{1}$, the reduced matrix element in general is given by

$$
\begin{aligned}
\left\langle\alpha, j_{1} j_{2} J\left\|\mathcal{T}^{(k)}(1)\right\| \alpha^{\prime}, j_{1}^{\prime} j_{2}^{\prime} J^{\prime}\right\rangle= & \delta_{j_{2}, j_{2}^{\prime}}(-1)^{j_{1}+j_{2}+J^{\prime}+k} \\
& \times \sqrt{J(J+1) J^{\prime}\left(J^{\prime}+1\right)}\left\{\begin{array}{ccc}
J & k & J^{\prime} \\
j_{1}^{\prime} & j_{2} & j_{1}
\end{array}\right\} \\
& \times\left\langle\alpha, j_{1}\left\|\mathcal{T}^{(1)}\right\| \alpha^{\prime}, j_{1}^{\prime}\right\rangle,
\end{aligned}
$$

where the braces represent the Wigner $6 j$ symbol (see, e.g. Ref. [107] for a derivation of (A.22) and general recursion relations and special cases of the $6 j$ symbol). In our case, with $k=1, j_{1}=j_{1}^{\prime}=L$ and $j_{2}=j_{2}^{\prime}=S$, Equation (A.21) reduces to

$$
\begin{aligned}
\left\langle L S J M_{J}\left|L_{0}^{(1)}\right| L S J^{\prime} M^{\prime}\right\rangle & =(-1)^{J-M+L+S+J^{\prime}+1} \times \sqrt{J(J+1) J^{\prime}\left(J^{\prime}+1\right)} \\
& \times\left(\begin{array}{ccc}
J & k & J^{\prime} \\
-M & q & M^{\prime}
\end{array}\right)\left\{\begin{array}{lll}
J & 1 & J^{\prime} \\
L & S & L
\end{array}\right\} \\
& \times\left\langle L \| L^{(1)}|| L\right\rangle .
\end{aligned}
$$


The calculation of the final reduced matrix element can now be done explicitly. By the Wigner-Eckart theorem we have

$$
\left\langle L M_{L}\left|L_{0}^{(1)}\right| L M_{L}^{\prime}\right\rangle=(-1)^{L-M_{L}}\left(\begin{array}{ccc}
L & 1 & L \\
M_{L} & 0 & M_{L}^{\prime}
\end{array}\right)\left\langle L\left\|L^{(1)}\right\| L\right\rangle .
$$

If we choose $M_{L}=M_{L}^{\prime}$, we can calculate both the matrix element on the left of Eq. (A.24) and $3 j$ symbol explicitly using the formula [107]

$$
\left(\begin{array}{ccc}
a & a & 1 \\
\beta & -\beta & 0
\end{array}\right)=(-1)^{a-\beta} \frac{\beta}{[a(a+1)(2 a+1)]^{1 / 2}} .
$$

The $3 j$ symbol is invariant under even permutations of the columns, thus we can put Eq. (A.24) into the form of Eq. (A.25). Plugging in values of $M_{L}=M_{L^{\prime}}$ we find

$$
\left\langle L M_{L}\left|L_{0}^{(1)}\right| L M_{L}\right\rangle=M_{L}=\left(\begin{array}{ccc}
L & L & 1 \\
M_{L} & -M_{L} & 0
\end{array}\right)\left\langle L|| L^{(1)}|| L\right\rangle
$$

where we have used the fact that $L_{z}\left|L M_{L}\right\rangle=M_{L}\left|L M_{L}\right\rangle$. We can thus solve for the reduced matrix element:

$$
\left\langle L|| L^{(1)}|| L\right\rangle=\sqrt{L(L+1)(2 L+1)}
$$

The final matrix element can now be expressed using the fact that the $6 j$ symbol is invariant under any permutation of the columns, and invariant under permutations of upper and lower arguments in any two columns:

$$
\begin{aligned}
\left\langle L S J M_{J}\left|L_{0}^{(1)}\right| L S J^{\prime} M_{J}^{\prime}\right\rangle & =(-1)^{J-M+L+S+J^{\prime}+1} \times \sqrt{J(J+1) J^{\prime}\left(J^{\prime}+1\right)} \\
& \times\left(\begin{array}{ccc}
J & k & J^{\prime} \\
-M & q & M^{\prime}
\end{array}\right)\left\{\begin{array}{ccc}
L & L & 1 \\
J & J^{\prime} & S
\end{array}\right\} \sqrt{L(L+1)(2 L+1)}
\end{aligned}
$$

This is the same as Equation 4.12. The same procedure can be used to calculate the matrix elements of $S_{z}$, given in equation 4.11 . 
For the calculation of the crystal field matrix elements (Equation 4.19) a slightly more complicated reduced matrix element must be calculated. It is given by

$$
\left\langle\Psi\left(4 f^{n}\right) L\left\|\sum_{i} r_{i}^{k} Y^{k}\right\| \Psi\left(4 f^{n}\right) L\right\rangle=\left\langle 4 f\left|r^{k}\right| 4 f\right\rangle\left\langle f^{n} L\left\|\sum_{i} Y^{k}\left(\theta_{i}, \phi_{i}\right)\right\| f^{n} L\right\rangle,
$$

where in Chapter 4 we used the notation $\left\langle r^{k}\right\rangle=\left\langle 4 f\left|r^{k}\right| 4 f\right\rangle$. The difficulty in this case is that $\sum_{i} Y^{k}\left(\theta_{i}, \phi_{i}\right)$ acts on single particle states, so the multielectron state $\left|f^{n} L\right\rangle$ must be expanded into single particle states consistent with antisymmetry under interchange of any two electrons. This expansion is done using coefficients of fractional parentage (cfp), describe in detail in Cowan [21]. However, because calculations using cfp are tedious, the matrix elements of the form (A.29) have been tabulated for all $p, d$ and $f$ shell configurations in Ref. [109]. The calculations of such reduced matrix elements are given in terms of a unit tensor operator

$$
\mathcal{U}^{(k)} \equiv \sum_{i=1} \mathfrak{u}^{(k)}(i)
$$

related to spherical harmonics by

$$
Y_{q}^{k}=\left\langle l|| Y^{k}|| l^{\prime}\right\rangle \mathfrak{u}_{q}^{(k)}
$$

and with the property $\left\langle l|| \mathfrak{u}^{(k)}|| l^{\prime}\right\rangle=1$ (here $l$ refers to a single particle orbital quantum number, with $l=3$ for the $4 f$ shell). In this way the reduced matrix elements may be determined by

$$
\left\langle\Psi\left(4 f^{n}\right) L \| \sum_{i} r_{i}^{k} Y^{k}|| \Psi\left(4 f^{n}\right) L\right\rangle=\left\langle 4 f\left|r^{k}\right| 4 f\right\rangle\left\langle L|| \mathcal{U}^{(k)}|| L\right\rangle\left\langle f \| Y^{k}|| f\right\rangle
$$

Each separate expectation value can then be found in tables. As noted in Chapter 4, the radial integrals $\left\langle 4 f\left|r^{k}\right| 4 f\right\rangle$ have been calculated in HartreeFock and Dirac-Fock schemes $[69,110]$. Values of $\left\langle l|| C^{k}|| l^{\prime}\right\rangle$ (where $C^{k}=$ $\left.[4 \pi /(2 k+1)]^{1 / 2} Y^{k}\right)$ are tabulated in Cowan [21]. The matrix elements of Equation (A.29) have conveniently been calculated in [35] for the Hund's rules 


$$
\begin{aligned}
& \frac{\text { Values of }\left\langle(4 f)^{n} L\left\|\sum_{k} r_{i}^{k} Y^{K}\right\|(4 f)^{n} L\right\rangle \text { in units of }}{k}\left\langle r^{k}\right\rangle
\end{aligned}
$$

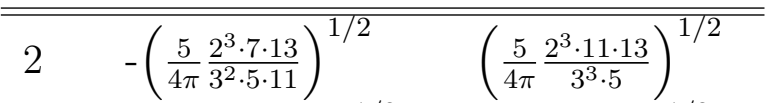

$$
\begin{aligned}
& 4-\left(\frac{9}{16 \pi} \frac{2^{8} \cdot 7 \cdot 13 \cdot 17}{3^{2} \cdot 11^{3}}\right)^{1 / 2} \quad\left(\frac{9}{16 \pi} \frac{2^{9} \cdot 13}{3^{2} \cdot 11}\right)^{1 / 2} \\
& 6-\left(\frac{13}{32 \pi} \frac{2^{10} \cdot 5^{4} \cdot 17 \cdot 19}{3^{2} \cdot 11^{3} \cdot 13}\right)^{1 / 2}-\left(\frac{13}{32 \pi} \frac{2^{10} \cdot 5^{3} \cdot 17}{3^{3} \cdot 11 \cdot 13}\right)^{1 / 2}
\end{aligned}
$$

Table A.1: Reduced matrix elements for $\mathrm{Sm}^{3+}$ and $\mathrm{Nd}^{3+}$ ions, taken from Ref. [35]

ground states of all the rare-earths, and are tabulated (in terms of $\left\langle r^{k}\right\rangle$ ), reproduced in Table A.1 for $\mathrm{Sm}^{3+}$ and $\mathrm{Nd}^{3+}$. 
Appendix A Matrix Elements of Irreducible Tensor Operators 


\section{Appendix B}

\section{Free Energy Minimization}

Here we derive the twisted magnetization profile of Chapter 5 by minimizing the free energy. The solution to the formally equivalent case of the exchangespring was given by Goto et al. [38], however no proof was given. Here we show how to determine the function $\theta(z)$ which minimizes the free energy using the calculus of variations. The basic methods of the calculus of variations can be found in, e.g. Refs. [111, 112]. We first present the general method of minimizing a functional with mixed boundary conditions.

\section{B.1 Extremizing a Functional}

The archetypal example of the calculus of variations involves finding the function $u(x)$ which minimizes (or extremizes) the functional $J[u]$. Here a functional is a map from the space of functions to a scalar in $\mathbb{R}$.

We assume $J$ is given by the following:

$$
J[u]=\int_{a}^{b} d x L\left(x, u, u^{\prime}\right)
$$

with the boundary conditions $u(a)=\alpha$ and $u(b)=\beta$. The first order variation of $u$ is given by $u_{\epsilon}(x)=u(x)+\epsilon w(x)$. If we force the variation to satisfy the boundary conditions (i.e. "fixed" boundary conditions) $u_{\epsilon}(a)=\alpha$ and $u_{\epsilon}(b)=\beta$, we have $w(a)=w(b)=0$. Carrying out the minimization (or extremization) procedure by setting the functional derivative equal to zero, we 
find

$$
\begin{aligned}
0 & =\left.\frac{d}{d \epsilon} J[u+\epsilon w]\right|_{\epsilon=0}, \\
& =\int_{a}^{b} d x w(x)\left(\frac{\partial L}{\partial u}-\frac{d}{d x} \frac{\partial L}{\partial u^{\prime}}\right)+\left[w(x) \frac{\partial L}{\partial u^{\prime}}\right]_{a}^{b}, \\
& =\int_{b}^{a} d x w(x)\left(\frac{\partial L}{\partial u}-\frac{d}{d x} \frac{\partial L}{\partial u^{\prime}}\right)+\left.w(b) \frac{\partial L}{\partial u^{\prime}}\right|_{x=b}-\left.w(a) \frac{\partial L}{\partial u^{\prime}}\right|_{x=a} .
\end{aligned}
$$

The last two terms are zero because $w(a)=w(b)=0$ and we recover the Euler-Lagrange equations:

$$
\frac{\partial L}{\partial u}-\frac{d}{d x} \frac{\partial L}{\partial u^{\prime}}=0
$$

When one boundary is left free, i.e. we allow variations of $w(x)$ that don't vanish at $x=b$ (in addition to those that vanish at $a$ and $b$ ), the term $w(b) \partial L /\left.\partial u^{\prime}\right|_{x=b}$ vanishes for arbitrary $w$ only if

$$
\left.\frac{\partial L}{\partial u^{\prime}}\right|_{x=b}=0
$$

In situation of Chapter 5, the functional to be minimized is

$$
E[\theta]=\int_{0}^{L} \mathrm{~d} z\left[A\left(\frac{d \theta(z)}{d z}\right)^{2}+M_{s} H \cos \theta(z)\right]
$$

Using the variations $\theta_{\epsilon}(z)=\theta(z)+\epsilon w(z)$ we have the boundary conditions $\theta(0)=0$ and we allow arbitrary variations at $z=L$, i.e. $\theta(L)$ is left free to be whatever minimizes $E$. Thus, in addition to satisfying the Euler-Lagrange equations, $\theta(z)$ must also satisfy the additional boundary condition

$$
\begin{aligned}
\left.\frac{\partial L}{\partial \theta^{\prime}}\right|_{L} & =\left.2 A \frac{d \theta(z)}{d z}\right|_{L}=0 \\
\left.\Longrightarrow \frac{d \theta(z)}{d z}\right|_{L} & =0
\end{aligned}
$$


However, because $\partial L / \partial z=0$ (a translational invariance symmetry), Noether's theorem guarantees there is a conserved quantity. The conserved quantity is given by

$$
\theta^{\prime} \frac{\partial L}{\partial \theta^{\prime}}-L=\text { const. }
$$

This reduces the order of the differential equation in $\theta(z)$ by one, and thus provides a simpler method of solution compared to solving the Euler-Lagrange equations directly.

\section{B.2 Explicit Solution for the Twisted Phase}

Making use of the conserved quantity of Eq. (B.10), we see that for the form of $L$ given in Eq. (B.7), we have

$$
\left(\theta^{\prime}\right)^{2}-\lambda^{2} \cos \theta(z)=\text { const. }
$$

where $\lambda^{2}=M_{s} H / A$. Using the boundary condition $\theta^{\prime}(L)=0$ immediately identifies the constant as $-\lambda^{2} \cos \theta(L) \equiv-\lambda^{2} \cos \theta_{0}$. After some algebra and integration, we find

$$
z \lambda=\int_{0}^{\theta(z)} \frac{\mathrm{d} \tilde{\theta}}{\sqrt{\cos \tilde{\theta}-\cos \theta_{0}}} .
$$

Using the identity $\cos \phi=1-2 \sin ^{2}(\phi / 2)$ and defining $k=\sin \left(\theta_{0} / 2\right)$, we arrive at

$$
z \lambda=\frac{1}{\sqrt{2}} \int_{0}^{\theta(z)} \frac{\mathrm{d} \tilde{\theta}}{\sqrt{k^{2}-\sin ^{2}(\tilde{\theta} / 2)}} .
$$

Finally, we make the substitution $\xi=k^{-1} \sin ^{2}(\tilde{\theta} / 2)$ and find

$$
z \alpha=\int_{0}^{k^{-1} \sin (\theta(z) / 2)} \frac{\mathrm{d} \xi}{\sqrt{\left(1-\xi^{2}\right)\left(1-k^{2} \xi^{2}\right)}},
$$

where $\alpha=\lambda / \sqrt{2}=\sqrt{M_{s} H / 2 A}$. This integral is an incomplete elliptic function of the first kind. It can be inverted by the use of the Jacobi elliptic function 
sn in the following way:

$$
\operatorname{sn}(z \alpha)=\frac{\sin \frac{\theta(z)}{2}}{k}=\frac{\sin \frac{\theta(z)}{2}}{\sin \frac{\theta(L)}{2}}
$$

When $z=L$, we find

$$
L \alpha=\int_{0}^{1} \frac{\mathrm{d} \xi}{\sqrt{\left(1-\xi^{2}\right)\left(1-k^{2} \xi^{2}\right)}}=K(k),
$$

where $K(k)$ is the complete elliptic function of the first kind, with module $k$ (see, e.g., Abramowitz \& Stegun [113] for properties of elliptic functions and Jacobi elliptic functions). We note that $K(k) \geqslant \pi / 2$ for $|k| \leqslant 1$, and so in order to obtain a non-trivial solution, we must have $L \sqrt{M_{s} H / 2 A} \geqslant \pi / 2$ for a twisted phase to exist. The explicit solution of $\theta(z)$ is given by

$$
\theta(z)=2 \sin ^{-1}\left(K^{-1}(\alpha L) \operatorname{sn}(z \alpha)\right) \quad \alpha=\sqrt{\frac{M_{s} H}{2 A}},
$$

where $K^{-1}$ is the inverse of the complete elliptic function of the first kind. While complete elliptic functions are found in standard computational packages, $K^{-1}$ is generally not among the built-in function. In this thesis we inverted $K(k)$ by using the Newton-Raphson method to find the roots $k^{*}$ of $K(k)-\alpha L=0$, where successive approximations of the roots are made by $k_{n+1}^{*}=k_{n}^{*}-K\left(k_{n}\right) / K^{\prime}\left(k_{n}\right)$, until suitable convergence is reached. The derivative of $K(k)$ is given by

$$
K^{\prime}(k)=\frac{d K}{d k}=\frac{E(k)}{k\left(1-k^{2}\right)}-\frac{K(k)}{k},
$$

where $E(k)$ is the complete elliptic function of the second kind, defined by

$$
E(k)=\int_{0}^{1} \mathrm{~d} \xi \frac{\sqrt{1-k^{2} \xi^{2}}}{\sqrt{1-\xi^{2}}} .
$$

Numerical schemes for calculating the functions $(K(k), E(k), \operatorname{sn}(u))$ can be found in Ref. [113], and are also found as built-in functions for many compu- 
B.2 Explicit Solution for the Twisted Phase tational packages. 
Appendix B Free Energy Minimization 


\section{References}

1. Aerts, C. M., Strange, P., Horne, M., et al. Half-metallic to insulating behavior of rare-earth nitrides. Phys. Rev. B 69, 045115 (2004).

2. Natali, F., Ruck, B. J., Plank, N. O. V., et al. Rare-earth mononitrides. Prog. Mater Sci. 58, 1316 -1360 (2013).

3. Leuenberger, F., Parge, A., Felsch, W., et al. GdN thin films: Bulk and local electronic and magnetic properties. Phys. Rev. B 72, 014427 (2005).

4. Granville, S., Ruck, B. J., Budde, F., et al. Semiconducting ground state of GdN thin films. Phys. Rev. B 73, 235335 (2006).

5. Preston, A. R. H, Granville, S., Housden, D. H., et al. Comparison between experiment and calculated band structures for DyN and $\mathrm{SmN}$. Phys. Rev. B 76, 245120 (2007).

6. Meyer, C., Ruck, B. J., Preston, A. R. H., et al. Magnetic properties of ErN films. J. Magn. Magn. Mater. 322, 1973 -1978 (2010).

7. Azeem, M., Ruck, B. J., Le Binh, D., et al. Optical response of DyN. J. Appl. Phys. 113, 203509 (2013).

8. Le Binh, D., Ruck, B. J., Natali, F., et al. Europium Nitride: A Novel Diluted Magnetic Semiconductor. Phys. Rev. Lett. 111, 167206 (2013).

9. Dietl, T. A ten-year perspective on dilute magnetic semiconductors and oxides. Nat. Mater. 9, 965-74 (2010).

10. Plank, N. O. V., Natali, F, Galipaud, J, et al. Enhanced Curie temperature in N-deficient GdN. Appl. Phys. Lett. 98, 112503 (2011). 


\section{REFERENCES}

11. Warring, H., Ruck, B., Trodahl, H. \& Natali, F. Electric field and photoexcited control of the carrier concentration in GdN. Appl. Phys. Lett. 102, 132409 (2013).

12. Natali, F., Ruck, B. J., Trodahl, H. J., et al. Role of magnetic polarons in ferromagnetic GdN. Phys. Rev. B 87, 035202 (2013).

13. Lee, C.-M., Warring, H, Vézian, S, et al. Highly resistive epitaxial Mgdoped GdN thin films. Appl. Phys. Lett. 106, 022401 (2015).

14. Larson, P., Lambrecht, W. R. L., Chantis, A. \& van Schilfgaarde, M. Electronic structure of rare-earth nitrides using the LSDA $+U$ approach: Importance of allowing $4 f$ orbitals to break the cubic crystal symmetry. Phys. Rev. B 75, 045114 (2007).

15. Senapati, K., Blamire, M. G. \& Barber, Z. H. Spin-filter Josephson junctions. Nat. Mater. 10, 849-852 (2011).

16. Muduli, P. K., Pal, A. \& Blamire, M. G. Crossover from diffusive to tunneling regime in $\mathrm{NbN} / \mathrm{DyN} / \mathrm{NbN}$ ferromagnetic semiconductor tunnel junctions. Phys. Rev. B 89, 094414 (2014).

17. Awschalom, D. D. \& Flatté, M. E. Challenges for semiconductor spintronics. Nat. Phys. 3, 153-159 (2007).

18. Morari, C, Beiuşeanu, F, Di Marco, I., et al. Magnetism and electronic structure calculation of SmN. J. Phys.: Condens. Matter 27, 115503 (2015).

19. Anton, E.-M., Ruck, B. J., Meyer, C., et al. Spin/orbit moment imbalance in the near-zero moment ferromagnetic semiconductor SmN. Phys. Rev. B 87, 134414 (2013).

20. McNulty, J. F., Anton, E.-M., Ruck, B. J., et al. Twisted phase of the orbital-dominant ferromagnet $\mathrm{SmN}$ in a GdN/SmN heterostructure. Phys. Rev. B 91, 174426 (2015).

21. Cowan, R. D. The theory of atomic structure and spectra (Univ of California Press, 1981). 
22. Nolting, W. \& Ramakanth, A. Quantum theory of magnetism (Springer, 2009).

23. Ashcroft, N. W. \& Mermin, N. D. Solid State Physics (Holt, Rinehart and Winston, New York, 2005).

24. Landau, L. D. \& Lifshitz, E. M. Quantum mechanics: non-relativistic theory (Elsevier, 2013).

25. Berestetskii, V. B., Lifshitz, E. M. \& Pitaevskii, L. P. Quantum electrodynamics (Butterworth-Heinemann, 1982).

26. Jensen, J. \& Mackintosh, A. R. Rare earth magnetism (Clarendon Oxford, 1991).

27. Wang, Y.-L. \& Cooper, B. R. Crystal-Field Effects and Anomalous Susceptibility of Antiferromagnets: Application to Ce-Group-V Compounds. Phys. Rev. B 2, 2607-2617 (1970).

28. Duan, C.-G., Sabirianov, R. F., Mei, W. N., et al. Electronic, magnetic and transport properties of rare-earth monopnictides. J. Phys.: Condens. Matter 19, 315220 (2007).

29. Adachi, H., Ino, H. \& Miwa, H. Separation of the $4 f$-spin, $4 f$-orbital, and conduction-electron magnetization from exotic thermomagnetic behavior for ferromagnetic Sm intermetallics. Phys. Rev. B 59, 1144511449 (1999).

30. Meyer, C., Ruck, B. J., Zhong, J., et al. Near-zero-moment ferromagnetism in the semiconductor SmN. Phys. Rev. B 78, 174406 (2008).

31. Bethe, H. A. Splitting of terms in crystals. Ann. Physik 3, 133-208 (1929).

32. Sakurai, J. J. \& Tuan, S. F. Modern quantum mechanics (AddisonWesley Reading, Massachusetts, 1985).

33. Goodenough, J. B. et al. Magnetism and the chemical bond (Interscience Publishers, New York, 1963).

34. Hutchings, M. T. Point-charge calculations of energy levels of magnetic ions in crystalline electric fields. Solid state physics 16, 227-273 (1964). 


\section{REFERENCES}

35. De Wijn, H., Van Diepen, A. \& Buschow, K. Samarium in Crystal Fields, a Case of Strong J-Mixing. Phys. Status Solidi (B) 76, 11-30 (1976).

36. Newman, D. J. \& Ng, B. The superposition model of crystal fields. Reports on Progress in Physics 52, 699 (1989).

37. Dresselhaus, M. S., Dresselhaus, G. \& Jorio, A. Group theory: application to the physics of condensed matter (Springer Science \& Business Media, 2007).

38. Goto, E., Hayashi, N., Miyashita, T. \& Nakagawa, K. Magnetization and Switching Characteristics of Composite Thin Magnetic Films. J. Appl. Phys. 36, 2951 (1965).

39. Skomski, R. Simple models of magnetism (Oxford University Press Oxford, 2008).

40. Thompson, A, Attwood, D., Gullikson, E., et al. X-ray data booklet (2009). URL http://xdb. lbl. gov (2009).

41. Stöhr, J. \& Siegmann, H. C. Magnetism: from fundamentals to nanoscale dynamics (Springer, 2007).

42. De Groot, F. \& Kotani, A. Core level spectroscopy of solids (CRC press, 2008).

43. Preston, A. R. H. Electronic structure of the rare-earth nitrides $\mathrm{PhD}$ thesis (Victoria University of Wellington, 2010).

44. Gusmeroli, R. \& Dallera, C. Missing. URL www.esrf.eu/UsersAndScience/ Experiments/TBS/SciSoft/OurSoftware/MISSING.

45. Thole, B. T., van der Laan, G., Fuggle, J. C., et al. 3d x-ray-absorption lines and the $3 d^{9} 4 f^{n+1}$ multiplets of the lanthanides. Phys. Rev. B 32, 5107-5118 (1985).

46. Richter, J., Ruck, B., Simpson, M., et al. Electronic structure of EuN: Growth, spectroscopy, and theory. Phys. Rev. B 84, 235120 (2011).

47. Nakajima, R., Stöhr, J. \& Idzerda, Y. Electron-yield saturation effects in L-edge x-ray magnetic circular dichroism spectra of $\mathrm{Fe}, \mathrm{Co}$, and $\mathrm{Ni}$. Phys. Rev. B 59, 6421-6429 (1999). 
48. Van der Laan, G. \& Figueroa, A. I. X-ray magnetic circular dichroismA versatile tool to study magnetism. Coord. Chem. Rev. (2014).

49. Harmon, B. N. \& Freeman, A. J. Spin-polarized energy-band structure, conduction-electron polarization, spin densities, and the neutron magnetic form factor of ferromagnetic gadolinium. Phys. Rev. B 10, 19791993 (1974).

50. Jo, T. \& Imada, S. 4f-5d Exchange Interaction and Magnetic Circular Dichroism in $\mathrm{L}_{2,3}$ Absorption for Ferromagnetic Rare Earths. J. Phys. Soc. Jpn. 62, 3721-3727 (1993).

51. Matsuyama, H., Harada, I. \& Kotani, A. Roles of 4f-5d Exchange Interactions in Magnetic Circular X-Ray Dichroism at the Rare-Earth $L_{2,3}$ Edges. J. Phys. Soc. Jpn. 66, 337-340 (1997).

52. Van Veenendaal, M., Goedkoop, J. B. \& Thole, B. T. Branching Ratios of the Circular Dichroism at Rare Earth $L_{23}$ Edges. Phys. Rev. Lett. 78, 1162-1165 (1997).

53. Parlebas, J., Asakura, K., Fujiwara, A., et al. X-ray magnetic circular dichroism at rare-earth $\mathrm{L}_{2,3}$ absorption edges in various compounds and alloys. Physics Reports 431, 1 -38 (2006).

54. Goedkoop, J. B., Thole, B. T., van der Laan, G., et al. Calculations of magnetic x-ray dichroism in the $3 d$ absorption spectra of rare-earth compounds. Phys. Rev. B 37, 2086-2093 (1988).

55. Thole, B. T., Carra, P., Sette, F. \& van der Laan, G. X-ray circular dichroism as a probe of orbital magnetization. Phys. Rev. Lett. 68, 19431946 (1992).

56. Carra, P., Thole, B. T., Altarelli, M. \& Wang, X. X-ray circular dichroism and local magnetic fields. Phys. Rev. Lett. 70, 694-697 (1993).

57. Natali, F., Ludbrook, B., Galipaud, J., et al. Epitaxial growth and properties of GdN, EuN and SmN thin films. Phys. Status Solidi (C) 9, 605608 (2012). 


\section{REFERENCES}

58. Buschow, K. H. J., van Diepen, A. M. \& de Wijn, H. W. Moment Reduction in Magnetically Ordered Samarium Intermetallics. Phys. Rev. B 8, 5134-5138 (1973).

59. De Wijn, H. W., van Diepen, A. M. \& Buschow, K. H. J. Effect of Crystal Fields on the Magnetic Properties of Samarium Intermetallic Compounds. Phys. Rev. B 7, 524-533 (1973).

60. Van Diepen, A. M., de Wijn, H. W. \& Buschow, K. H. J. Temperature Dependence of the Crystal-Field-Induced Anisotropy in $\mathrm{SmFe}_{2}$. Phys. Rev. B 8, 1125-1129 (1973).

61. Adachi, H., Ino, H. \& Miwa, H. Effect of conduction-electron polarization on the magnetism of hcp samarium metal. Phys. Rev. B 56, 349354 (1997).

62. Adachi, H \& Ino, H. A ferromagnet having no net magnetic moment. Nature 401, 148-150 (1999).

63. Adachi, H., Kawata, H., Hashimoto, H., et al. Zero-Magnetization Ferromagnet Proven by Helicity-Switching Compton Scattering. Phys. Rev. Lett. 87, 127202 (2001).

64. Qiao, S., Kimura, A., Adachi, H., et al. Direct evidence of ferromagnetism without net magnetization observed by x-ray magnetic circular dichroism. Phys. Rev. B 70, 134418 (2004).

65. Dhesi, S. S., van der Laan, G., Bencok, P., et al. Spin- and orbitalmoment compensation in the zero-moment ferromagnet $\mathrm{Sm}_{0.974} \mathrm{Gd}_{0.026} \mathrm{Al}_{2}$. Phys. Rev. B 82, 180402 (2010).

66. Racah, G. Theory of Complex Spectra. II. Phys. Rev. 62, 438-462 (1942).

67. SymPy Development Team. SymPy: Python library for symbolic mathematics (2014).

68. Lea, K., Leask, M. \& Wolf, W. The raising of angular momentum degeneracy of $f$-electron terms by cubic crystal fields. Journal of Physics and Chemistry of Solids 23, 1381-1405 (1962). 
69. Freeman, A. J. \& Watson, R. E. Theoretical Investigation of Some Magnetic and Spectroscopic Properties of Rare-Earth Ions. Phys. Rev. 127, 2058-2075 (1962).

70. Cortie, D. L., Brown, J. D., Brück, S., et al. Intrinsic reduction of the ordered $4 f$ magnetic moments in semiconducting rare-earth nitride thin films: DyN, ErN, and HoN. Phys. Rev. B 89, 064424 (2014).

71. Hulliger, F. Rare earth pnictides. Handbook on the physics and chemistry of rare earths 4, 153-236 (1979).

72. Furrer, A. \& Halg, W. Crystal-field splittings of NdN and HoN. J. Phys. C: Solid State Phys. 9, 3499 (1976).

73. Schumacher, D. P. \& Wallace, W. E. Magnetic Characteristics of Some Lanthanide Nitrides. Inorg. Chem. 5, 1563-1567 (1966).

74. Lévy, F. Effets magnétostrictifs spontanés de quelques composés des terres rares. Physik der kondensierten Materie 10, 71-84 (1969).

75. Mourgout, C, Chevalier, B., Etourneau, J., et al. Les oxynitrures de néodyme NdN1-xOx. Préparation et étude des propriétés magnétiques, électriques et structurales. Revue Internationale des Hautes Temperatures et des Refractaires 14, 89-96 (1977).

76. Schobinger-Papamantellos, P, Fischer, P, Vogt, O \& Kaldis, E. Magnetic ordering of neodymium monopnictides determined by neutron diffraction. J. Phys. C: Solid State Phys. 6, 725 (1973).

77. Levy, P. M. \& Chen, H. H. Structural and Magnetic Phase Transitions in the Rare-Earth Pnictides. Phys. Rev. Lett. 27, 1385-1388 (1971).

78. Huang, H. L. \& Lin, M. Y. Remanent Magnetization in Randomly Distributed Cubic Particles. Phys. Status Solidi (A) 91, 123-127 (1985).

79. Schumacher, D. \& Hollingsworth, C. Fourth order crystal field splitting in rare earth (III) ions. J. Phys. Chem. Solids 27, 749 -753 (1966).

80. Ebisu, S., Morita, H. \& Nagata, S. Influence of cubic crystal field on the magnetic susceptibility of defect-perovskite $\mathrm{RT} a_{3} \mathrm{O}_{9}(\mathrm{R}=$ rare earth). $J$. Phys. Chem. Solids 61, $45-65$ (2000). 


\section{REFERENCES}

81. Thiaville, A. \& Fert, A. Twisted spin configurations in thin magnetic layers with interface anisotropy. J. Magn. Magn. Mater. 113, $161-172$ (1992).

82. Rößler, U. K., Bogdanov, A. N. \& Pfleiderer, C. Spontaneous skyrmion ground states in magnetic metals. Nature 442, 797-801 (2006).

83. Parkin, S. S., Hayashi, M. \& Thomas, L. Magnetic domain-wall racetrack memory. Science 320, 190-194 (2008).

84. Thiaville, A., Rohart, S., Jué, É., et al. Dynamics of Dzyaloshinskii domain walls in ultrathin magnetic films. Europhys. Lett. 100, 57002 (2012).

85. Emori, S., Bauer, U., Ahn, S.-M., et al. Current-driven dynamics of chiral ferromagnetic domain walls. Nat. Mater. 12, 611-616 (2013).

86. Khvalkovskiy, A. V., Cros, V., Apalkov, D., et al. Matching domain-wall configuration and spin-orbit torques for efficient domain-wall motion. Phys. Rev. B 87, 020402 (2013).

87. Zocher, H. The effect of a magnetic field on the nematic state. Trans. Faraday Soc. 29, 945-957 (1933).

88. Camley, R. E. Surface spin reorientation in thin Gd films on Fe in an applied magnetic field. Phys. Rev. B 35, 3608-3611 (1987).

89. Berkowitz, A. \& Takano, K. Exchange anisotropya review. J. Magn. Magn. Mater. 200, 552-570 (1999).

90. Bogdanov, A. N. \& Rößler, U. K. Magnetic-field-induced reorientation in thin antiferromagnetic films: Spin-flop transition and surface-induced twist effects. Phys. Rev. B 68, 012407 (2003).

91. Bogdanov, A. N. \& Rößler, U. K. Chiral Symmetry Breaking in Magnetic Thin Films and Multilayers. Phys. Rev. Lett. 87, 037203 (2001).

92. Ludbrook, B. M., Farrell, I. L., Kuebel, M., et al. Growth and properties of epitaxial GdN. J. Appl. Phys. 106, 063910 (2009). 
93. Fullerton, E. E., Jiang, J. S., Grimsditch, M., et al. Exchange-spring behavior in epitaxial hard/soft magnetic bilayers. Phys. Rev. B 58, 1219312200 (1998).

94. Camley, R. E. \& Tilley, D. R. Phase transitions in magnetic superlattices. Phys. Rev. B 37, 3413-3421 (1988).

95. Dufour, C., Cherifi, K., Marchal, G., et al. Polarized neutron scattering from Gd/Fe multilayers: Twisted phase and spin-flip scattering. Phys. Rev. B 47, 14572-14575 (1993).

96. Hahn, W., Loewenhaupt, M., Huang, Y. Y., et al. Experimental determination of the magnetic phase diagram of $\mathrm{Gd} / \mathrm{Fe}$ multilayers. Phys. Rev. B 52, 16041-16048 (1995).

97. Kravtsov, E., Haskel, D., te Velthuis, S. G. E., et al. Complementary polarized neutron and resonant x-ray magnetic reflectometry measurements in Fe/Gd heterostructures: Case of inhomogeneous intralayer magnetic structure. Phys. Rev. B 79, 134438 (2009).

98. Koizumi, A., Takagaki, M., Suzuki, M., et al. Anomalous magnetic hysteresis of Gd and Fe moments in a Gd/Fe multilayer measured by hard xray magnetic circular dichroism. Phys. Rev. B 61, 14909-14912 (2000).

99. Natali, F., Plank, N. O. V., Galipaud, J., et al. Epitaxial growth of GdN on silicon substrate using an AlN buffer layer. J. Cryst. Growth 312, $3583-3587$ (2010).

100. Van der Laan, G. \& Thole, B. T. X-ray-absorption sum rules in $j j$ coupled operators and ground-state moments of actinide ions. Phys. Rev. B 53, 14458-14469 (1996).

101. Bowden, G., Beaujour, J., Gordeev, S, et al. Discrete exchange-springs in magnetic multilayer samples. J. Phys.: Condens. Matter 12, 9335 (2000).

102. Nogués, J. \& Schuller, I. K. Exchange bias. J. Magn. Magn. Mater. 192, 203-232 (1999). 


\section{REFERENCES}

103. Leuenberger, F., Parge, A., Felsch, W., et al. X-ray magnetic circular dichroism at the $\operatorname{Gd} L_{2,3}$ absorption edges in GdN layers: The influence of lattice expansion. Phys. Rev. B 73, 214430 (2006).

104. Dumesnil, K, Dufour, C, Mangin, P., et al. Temperature dependence in the magnetization reversal process of $\mathrm{DyFe}_{2} / \mathrm{YFe}_{2}$ exchange-coupled superlattices. J. Phys.: Condens. Matter 17, 215 (2005).

105. Stenning, G. B. G., Bowden, G. J., de Groot, P. A. J., et al. Magnetic reversal in Dy-doped $\mathrm{DyFe}_{2} / \mathrm{YFe}_{2}$ superlattice films. Phys. Rev. B 91, 094403 (2015).

106. Gordeev, S. N., Beaujour, J.-M. L., Bowden, G. J., et al. Giant Magnetoresistance by Exchange Springs in $\mathrm{DyFe}_{2} / \mathrm{YFe}_{2}$ Superlattices. Phys. Rev. Lett. 87, 186808 (2001).

107. Brink, D. M. \& Satchler, G. R. Angular momentum (Oxford University Press, 1993).

108. Wybourne, B. G. \& Smentek, L. Optical spectroscopy of lanthanides: magnetic and hyperfine interactions (CRC Press, 2007).

109. Nielson, C. \& Koster, G. F. Spectroscopic Coefficients for the $p^{n}, d^{n}$, and $f^{n}$ Configurations (MIT press, 1963).

110. Freeman, A. \& Desclaux, J. Dirac-Fock studies of some electronic properties of rare-earth ions. J. Magn. Magn. Mater. 12, 11-21 (1979).

111. Arfken, G. B. Mathematical methods for physicists (Academic press, 2013).

112. Gelfand, I. M., Fomin, S. V. \& Silverman, R. A. Calculus of variations (Courier Corporation, 2000).

113. Abramowitz, M. \& Stegun, I. A. Handbook of mathematical functions: with formulas, graphs, and mathematical tables 55 (Courier Corporation, 1964). 